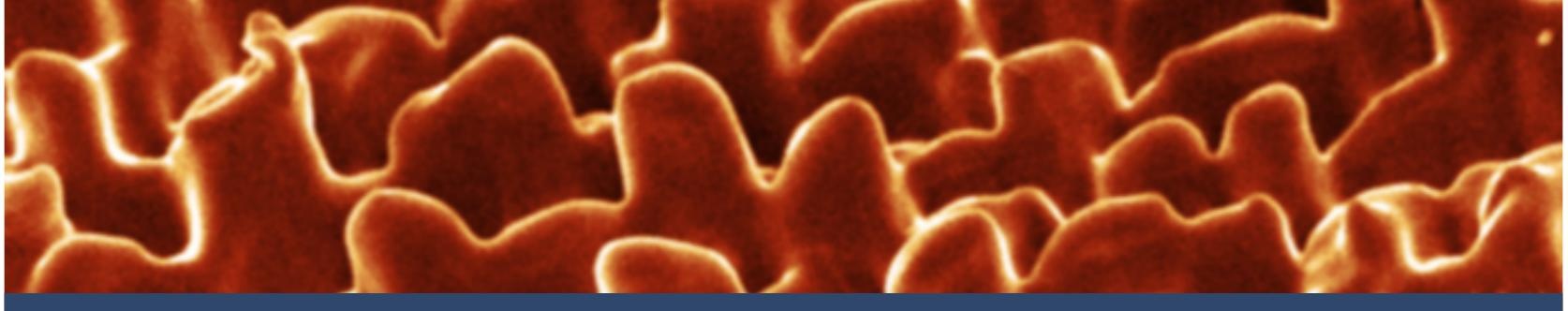

AT THE INTERFACE BETWEEN

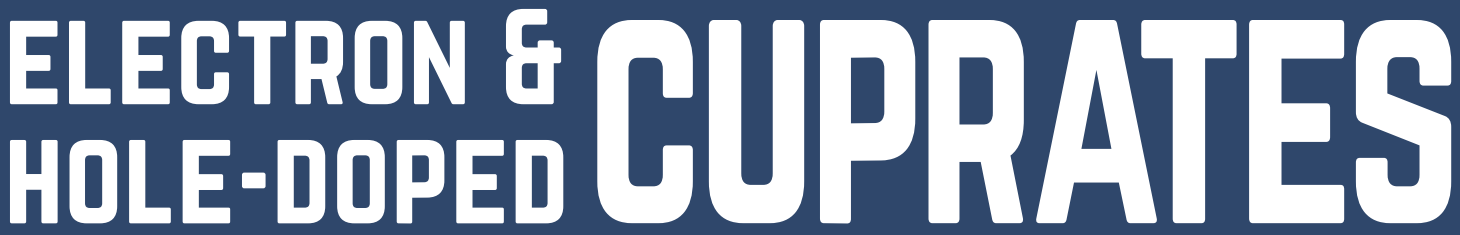

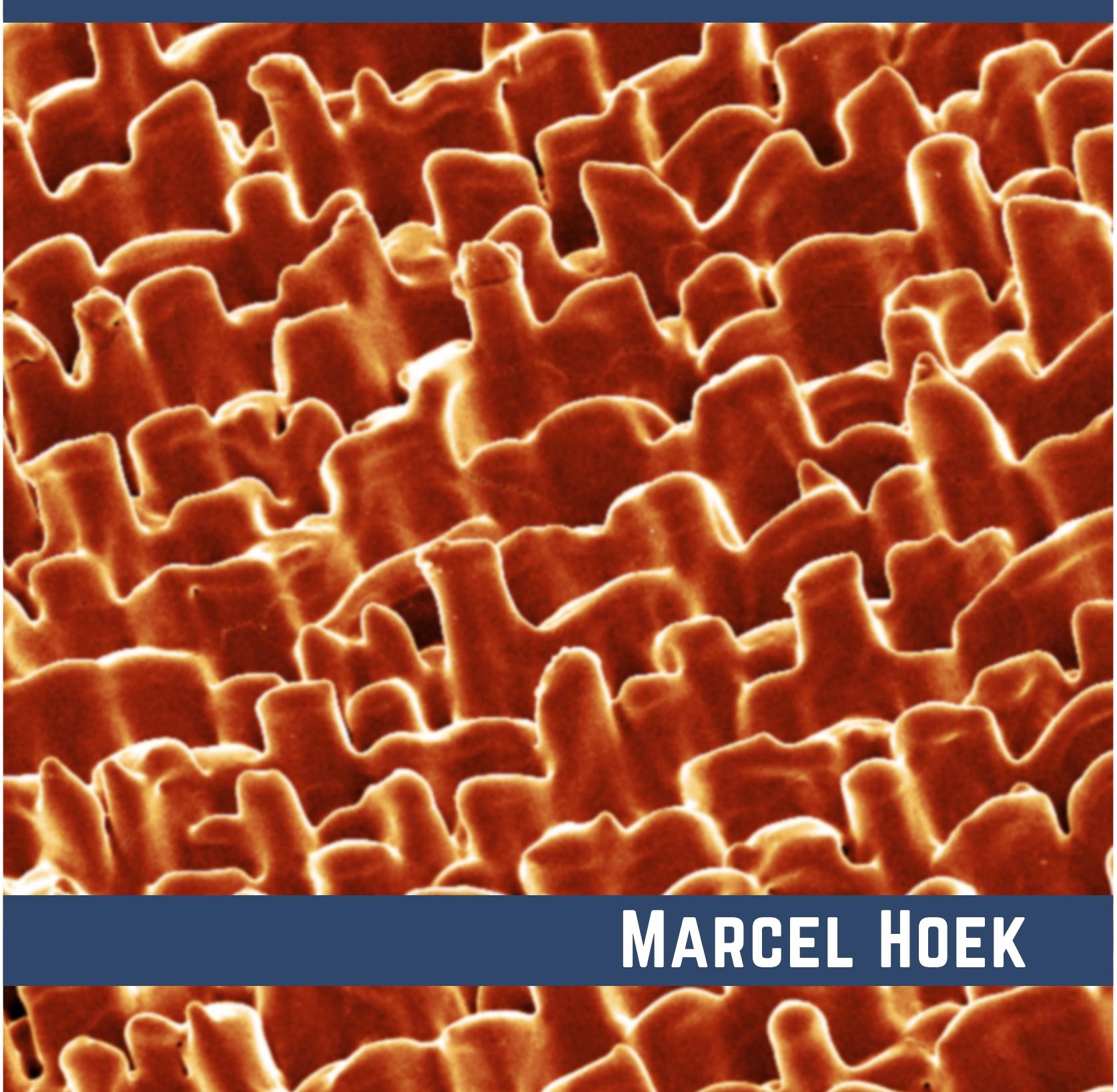



AT THE INTERFACE BETWEEN
ELECTRON E CUPRATES
HOLE-DOPED CUPRATE

MARCEL HOEK 


\section{Ph.D. committee}

Chairman the Dean of the faculty of Science and Technology University of Twente

\section{Supervisor}

prof. dr. ir. H. Hilgenkamp

University of Twente

Members

prof. dr. L. Alff

prof. dr. N.E. Hussey

prof. dr. J. Ye

prof. dr. ir. A. Brinkman

prof. dr. ir. W.G. van der Wiel

Technische Universität Darmstadt

Radboud University Nijmegen

University of Groningen

University of Twente

University of Twente

\section{Cover}

The cover shows a false color scanning electron microscope image (250 $\mathrm{\mu m}$ wide from front to back) of an eerie landscape of cones and ridges that forms on the surface of $\mathrm{YBa}_{2} \mathrm{Cu}_{3} \mathrm{O}_{7-\mathrm{x}}$ during pulsed laser ablation. With each pulse, the surface cracks and ripples due to large thermal stresses. The cones form as yttrium collects in the crests of the ripples, causing them to harden and protect the material underneath. The resulting landscape bears a striking resemblance to rock formations called Hoodoos, which form via a similar mechanism, but on a much larger scale.

\section{Acknowledgments}

The research described in this thesis was performed in the Faculty of Science and Technology and the MESA+ Institute for Nanotechnology at the University of Twente. The main work was financially supported by the Netherlands Organization for Scientific Research (NWO) under a VICI grant.

Some experiments were performed on the ID13 beamline at the European Synchrotron Radiation Facility (ESRF), Grenoble, France. We are grateful to Dr. Emanuela Di Cola at ESRF for providing assistance in using beamline ID13.

Part of the research leading to these results has received funding from the European Union Seventh Framework Programme under Grant Agreement 312483 - ESTEEM2 (Integrated Infrastructure Initiative-I3).

This thesis is typeset with LATEX using the fonts NORWESTER, created by Jamie Wilson, Cuprum, created by Jovanny Lemonad, and Latin Modern.

At the interface between electron and hole-doped cuprates

Ph.D. thesis, University of Twente, Enschede, The Netherlands

Printed by Gildeprint Drukkerijen

ISBN: 978-90-365-3699-8

DOI: $10.3990 / 1.9789036536998$

(C) M. Hoek, 2014 


\section{AT THE INTERFACE BETWEEN \\ ELECTRON AND HOLE-DOPED CUPRATES}

\section{PROEFSCHRIFT}

ter verkrijging van

de graad van doctor aan de Universiteit Twente, op gezag van de rector magnificus, prof. dr. H. Brinksma volgens besluit van het College voor Promoties in het openbaar te verdedigen op donderdag 28 augustus 2014 om 16:45 uur

door

Marcel Hoek

geboren op 10 december 1984

te Apeldoorn 
Dit proefschrift is goedgekeurd door de promotor:

prof. dr. ir. H. Hilgenkamp 


\section{Contents}

Introduction $\quad 1$

1 Combining electron and hole-doped cuprates 5

1.1 General properties . . . . . . . . . . . . . . 5

1.1.1 Crystal structure . . . . . . . . . . . . . 5

1.1.2 Phase diagram . . . . . . . . . . . . . . . . 6

1.2 Requirements for combination . . . . . . . . . . . . 8

1.2.1 Annealing procedure and oxygenation . . . . . . . . 8

1.2.2 Combinations of electron and hole-doped cuprates . . . . . 9

$1.2 .3 \mathrm{LSCO}$ and $\mathrm{NCCO} \ldots \ldots . . \ldots . . \ldots 10$

1.2.4 $\mathrm{La}_{2-\mathrm{x}} \mathrm{Ce}_{\mathrm{x}} \mathrm{CuO}_{4} \ldots \ldots \ldots \ldots$. . . . . . . . . . . . . . . . . . . . . . . . . . 10

1.2.5 Substrate choice . . . . . . . . . . . . . . 11

1.2.6 Contact resistance . . . . . . . . . . . . . . . . . 12

1.3 Semiconductor model for LSCO/NCCO junctions . . . . . . . . . . 14

1.3.1 General band diagram . . . . . . . . . . . . . 14

1.3.2 Anderson/Schottky-Mott band alignment . . . . . . . . 15

1.3.3 Valence band hybridization . . . . . . . . . . . . 15

1.3.4 Band bending . . . . . . . . . . . . . . 16

1.3.5 Thomas-Fermi screening length . . . . . . . . . . . 16

1.3.6 Depletion zone . . . . . . . . . . . . . . 18

1.4 Conclusions . . . . . . . . . . . . . . . . . . . . . . . . 19

2 Effect of high oxygen pressure annealing on $\mathrm{Nd}_{1.85} \mathrm{Ce}_{0.15} \mathrm{CuO}_{4} \quad 21$

2.1 Introduction . . . . . . . . . . . . . . . . . 21

2.2 Experimental details . . . . . . . . . . . . . . . . . . . . . . . . . 22

2.3 Results and discussion . . . . . . . . . . . . . . . . . . . . . 24

2.3.1 Suppression of the $(\mathrm{Nd}, \mathrm{Ce})_{2} \mathrm{O}_{3}$ parasitic phase . . . . . . 24

2.3.2 Effect of different annealing procedures . . . . . . . . . 27

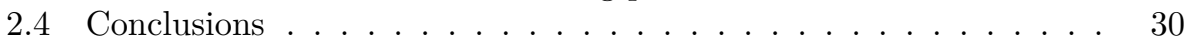

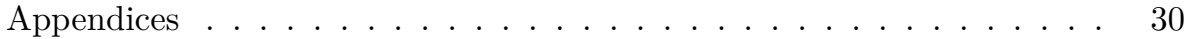

A Effect of oxygen annealing for different doping levels . . . . 30

3 LSCO/NCCO ramp-edge $p n$-junctions 33

3.1 Introduction . . . . . . . . . . . . . . . . . . . 33

3.2 Experimental details . . . . . . . . . . . . . . . . . . . . . . . . . . . . . . . . . 34

3.2.1 Sample fabrication . . . . . . . . . . . . . . 34

3.2.2 Structural characterization . . . . . . . . . . . . . . . . 35

3.2.3 Measurement setup . . . . . . . . . . . . . 39 
3.3 Results and discussion $\ldots \ldots \ldots \ldots \ldots \ldots$

$3.3 .1 \quad I V$ measurements . . . . . . . . . . . . . . . . . . . . 39

3.3.2 Tunneling spectrum with phonon modes . . . . . . . . . 41

3.3 .3 Origin of the barrier . . . . . . . . . . . . . . . 42

3.3.4 Disentangling strain and electronic effects . . . . . . . . . 44

3.3.5 Metal-insulator-metal modeling . . . . . . . . . . . . . . 45

3.3.6 $p n$-junctions with different interlayers $\ldots \ldots \ldots \ldots$

3.3 .7 Temperature dependence . . . . . . . . . . . . . 50

3.3.8 Absence of a Josephson contact . . . . . . . . . . . . 50

3.3.9 Effect of different interlayers . . . . . . . . . . . . 52

3.3.10 Disentangling strain and electronic effects, pt. II . . . . . 54

3.4 Conclusions . . . . . . . . . . . . . . . . . . . 54

Appendices ............................. 56

A Superconducting gap from phonon spectrum . . . . . . 56

B Fabrication and characterization of homojunctions . . . . 56

C LSCO on an etched substrate . . . . . . . . . . 57

4 Artificial domain boundary in LSCO/NCCO ramp-edge $p n$-junctions

4.1 Introduction . . . . . . . . . . . . . . . . . . . 59

4.2 Experimental details . . . . . . . . . . . . . . . . 60

4.2 .1 Sample fabrication . . . . . . . . . . . . . . 60

4.2 .2 HAADF-STEM . . . . . . . . . . . . . . . 60

4.2 .3 Scanning nano-focused X-ray diffraction . . . . . . . . . . 61

4.3 Results and discussion . . . . . . . . . . . . . . . . 63

4.3 .1 nXRD mapping . . . . . . . . . . . . . . . 63

4.3 .2 Tilted LSCO phase . . . . . . . . . . . . . . . . 65

4.3 .3 HAADF-STEM . . . . . . . . . . . . . . . 65

4.3.4 Origin of the LSCO lattice tilt . . . . . . . . . . 65

4.3.5 Model validation . . . . . . . . . . . . . . . . . 70

4.3.6 Other material combinations . . . . . . . . . . . . 70

4.4 Conclusions . . . . . . . . . . . . . . . . . . . 73

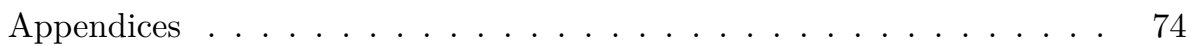

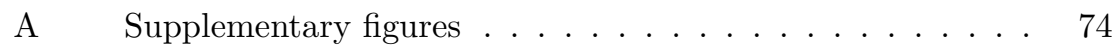

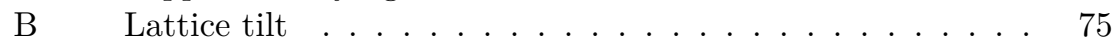

5 NCCO-LSCO bilayers and $c$-axis contacts $\quad \mathbf{7 7}$

5.1 Introduction . . . . . . . . . . . . . . . . . . 77

5.2 Deposition conditions . . . . . . . . . . . . . . . . 78

5.3 Sample characterization . . . . . . . . . . . . . . . . . 81

5.3 .1 X-ray diffraction . . . . . . . . . . . . . . . . 81

5.3 .2 HAADF-STEM and AFM . . . . . . . . . . . 84

5.4 Results and discussion . . . . . . . . . . . . . . . . 85

5.4 .1 In-plane transport . . . . . . . . . . . . . . . 85

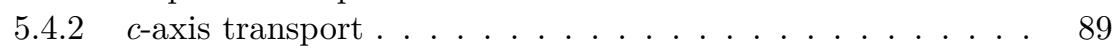

5.4 .3 X-ray absorption spectroscopy _............. 91 


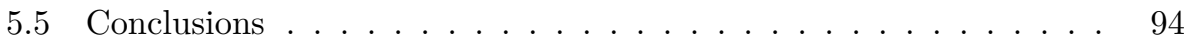

6 Towards a test for current quantization in type-II Bose-Mott $\begin{array}{lr}\text { insulators } & 97\end{array}$

6.1 Introduction . . . . . . . . . . . . . . . . . . . . . 97

6.2 Theoretical background . . . . . . . . . . . . . . . . . . 98

6.3 Experimental details . . . . . . . . . . . . . . . . . . . 103

6.4 Sample characterization . . . . . . . . . . . . . 106

6.5 Experimental results . . . . . . . . . . . . . . . . . 110

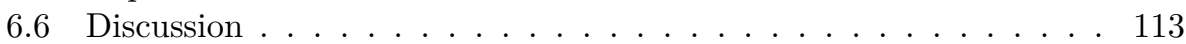

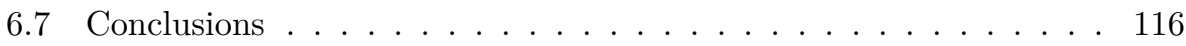

Appendices ............................ 116

A Current source limitations . . . . . . . . . . 116

$\begin{array}{lr}\text { Epilogue } & 119\end{array}$

$\begin{array}{lc}\text { Summary } & 123\end{array}$

$\begin{array}{lr}\text { Samenvatting } & 127\end{array}$

$\begin{array}{ll}\text { Dankwoord } & 131\end{array}$

$\begin{array}{ll}\text { References } & 133\end{array}$ 



\section{Introduction}

1986 was a good year for two reasons: it was the year high- $T_{c}$ superconductivity was discovered [1] and it gave me a little brother. I was not aware of the former at the time, but over the years I have come to appreciate both. The discovery of high- $T_{c}$ superconductivity in cuprates by Bednorz and Müller was remarkable, not just because of the high critical temperature $\left(T_{c}\right)$, but also because they were looking at oxides, materials that were famously on Bernd Matthias' list of materials that were not supposed to be superconducting, along with insulators and magnetic elements. It has led to a race to find ever more complex materials with ever higher $T_{c}$; the best known example being $\mathrm{YBa}_{2} \mathrm{Cu}_{3} \mathrm{O}_{7-\mathrm{x}}$, the first material to be superconducting above the temperature of liquid nitrogen [2]. Interestingly, now, over a quarter of a century later, there is still no consensus on a microscopic theory for high- $T_{c}$ superconductivity [3].

After the cuprates, oxides in general became the subject of research, in particular in thin film growth and in heterostructures $[4,5]$. In many (transition) metal oxides, the oxygen acts as a mediator between the localized $d$ or $f$ states of the metal atoms, leading to strong correlations that manifest in many ways, ranging from Mott insulators and superconductors, to all flavors of magnetism, piezoelectricity and multiferroics, and even more exotic variations like spin-ice and topological insulators. All these effects can be tailored by specific material choice, strain engineering, etc., and they can be combined in heterostructures, often resulting in new physics. A remarkable example of this is the appearance of a two-dimensional electron gas (2DEG) at the interface between two insulators $\mathrm{SrTiO}_{3}$ and $\mathrm{LaAlO}_{3}$ (LAO/STO) [6].

In many ways, the developments in the oxide research parallel the semiconductor research that took off in the seventies, which has culminated in the high mobility 2DEGs and the (fractional) quantum Hall effect we are now familiar with $[7,8]$. The properties of the oxides are currently tailored down to the orbital level [5] and there is a strong focus on eliminating defects [9]. This is leading to charge mobilities approaching those used in the original quantum Hall experiments [9].

In this spirit, we want to investigate how concepts from semiconductor physics translate to strongly correlated oxide materials. In particular, we will focus on the combination electron-doped cuprate $\mathrm{Nd}_{2-\mathrm{x}} \mathrm{Ce}_{\mathrm{x}} \mathrm{CuO}_{4}$ (NCCO) and the hole-doped cuprate $\mathrm{La}_{2-\mathrm{x}} \mathrm{Sr}_{\mathrm{x}} \mathrm{CuO}_{4}$ (LSCO) in a $p n$-junction analogue. For these materials, superconductivity appears upon Ce or Sr substitution. At zero doping, the parent compounds of NCCO and LSCO have half filled bands. Conventional band theory would make them metals, but the localized nature of the charge carriers and strong on-site Coulomb repulsion drive the system into an insulating state, the Mott 
insulator. The parallel between semiconductors and cuprates is easy to make, both materials are characterized by an energy gap and carrier doping occurs by substitution. However, since the energy gap appears due to strong correlations in the cuprates, there may be significant differences. This has inspired theoretical exploration into the semiconductor physics parallels that can be drawn, ranging from $p n$-junction effects such as Esaki diodes [10], Josephson diodes [11] and depletion zones with unusual properties [12], to effects related to exciton formation with strong localization of single excitons in the antiferromagnetic background of the cuprates at low doping [13], Bose-Einstein condensation and phase separation of various ordered phases at finite densities [14,15], and an enhanced thermoelectric figure of merit [16].

The formation of excitons is a tantalizing prospect. An exciton is a bound pair of an electron and a hole, that can form under the influence of a Coulomb interaction. The resulting particle is a boson. It has been predicted that excitons, like any other boson, can undergo Bose-Einstein condensation. Excitons are particularly prevalent in semiconductor systems. Advances in semiconductor 2DEG technology have made it possible to control the formation, motion and decay of excitons in semiconductor systems. Recently, optically created excitons were shown to condense in an electrostatic trap in a coupled quantum well structure [17]. Strong evidence for condensation is also found in parallel 2DEGs [18], in quantum Hall bilayers [19-21] and exciton-polariton gases [22-24], in which strong coherence effects are observed.

The charge carriers form a non-interacting gas in semiconductor systems; the question arises what would happen if strong correlations were introduced. Cuprates are a good candidate to study this question because: (1) strong correlations dominate the phase diagram and create a charge-transfer insulator state (Mott insulator) at zero doping; (2) they span a rich phase diagram as a function of both electron and hole-doping; and (3) the conductivity mainly occurs in quasi two-dimensional $\mathrm{CuO}_{2}$ planes. Excitonic effects can be expected between two oppositely doped $\mathrm{CuO}_{2}$ planes, as has been predicted for insulating YBCO $[13,25]$.

In this thesis, we study two oppositely doped cuprates, NCCO and LSCO, in close proximity. We show that these materials can be combined on a single chip, while maintaining superconductivity in both compounds.

Chapter 1 introduces relevant information about electron and hole-doped cuprates, and details the ingredients needed for the combination of oppositely doped cuprates on a single chip, in particular the conflicting oxygenation requirements for NCCO and LSCO. We apply concepts from semiconductor physics to get an estimate for the energy and length scales of electronic effects at the interface between NCCO and LSCO.

Next, chapter 2 looks at the oxygenation requirements in more detail. In this chapter we explore the effect of high oxygen pressure annealing on the electrondoped NCCO and we show how a copper poor parasitic phase plays an important role. 
Chapter 3 will focus on the in-plane combination of NCCO and LSCO using rampedge technology. Here, we discuss fabrication and structural characterization aspects, as well as electronic characterization. This allows us to test the theoretical predictions mentioned earlier and in chapter 1.

Chapter 4 is a more detailed study of a structural effect seen in the characterization of the devices in chapter 3. A tilting of the LSCO lattice on the NCCO rampedge is observed by high-angle annular dark-field transmission electron microscopy (HAADF-STEM) and nano-focused X-ray diffraction (nXRD). This tilt is explained using a strain accommodation model.

This is followed by chapter 5 , where we look at a $c$-axis contact between NCCO and LSCO. We characterize devices with two bilayer configurations, NCCO with LSCO on top and the reverse. The electronic properties are measured in-plane and in the $c$-axis direction. The local electronic structure of the LSCO in the first configuration is also measured using X-ray absorption spectroscopy (XAS), where we find remarkable differences between single LSCO films and LSCO in a bilayer configuration.

The last chapter, chapter 6 , moves away from the electron-hole combinations and focuses on the pseudogap phase of the hole-doped cuprates. This chapter details device fabrication using knowledge from chapter 3 towards a test for current quantization in a type-II Bose-Mott insulator. This is based on the theoretical prediction that a type-II Bose-Mott insulator exists as a dual state to a type-II superconductor, where vortices can appear as current lines instead of vortices of magnetic flux. A candidate for the type-II Bose-Mott insulator phase is the pseudogap phase in the hole-doped cuprates, which is suggested to consist of preformed Cooper pairs without long-range order.

The thesis ends with an epilogue on how the results discussed in this thesis can be used in the further study of exciton physics in doped Mott insulators, as well as a brief outlook on exciton physics in other strongly correlated materials and other unconventional materials. 



\section{CHAPTER 1}

\section{Combining electron and hole-doped cuprates}

This chapter deals with the basic ingredients needed to combine electron and hole-doped cuprates. It starts with the general properties of the cuprates, with a focus on electron-doped $\mathrm{Nd}_{2-\mathrm{x}} \mathrm{Ce}_{\mathrm{x}} \mathrm{CuO}_{4}$ (NCCO) and holedoped $\mathrm{La}_{2-\mathrm{x}} \mathrm{Sr}_{\mathrm{x}} \mathrm{CuO}_{4}$ (LSCO) that are used throughout this thesis. Next, challenges in the combination of oppositely doped cuprates are discussed, focusing on material choice, annealing requirements and contact resistance. Finally, the combination of NCCO and LSCO is approached using a semiconductor perspective. Two types of possible band alignments are presented, an Anderson/Schottky-Mott type alignment dictated by the work function of both materials, and a valence band hybridization scenario, where the inplane O $2 p$ orbitals dictate the band alignment. For both scenarios, the energy and length scale of the $p n$ depletion zone is estimated.

\subsection{General properties}

\subsubsection{Crystal structure}

Cuprates are characterized by a layered structure of $\mathrm{CuO}_{2}$ planes separated by cation layers. In the case of NCCO and LSCO, the crystal structure of the parent compounds $\mathrm{Re}_{2} \mathrm{CuO}_{4}$ (with $\mathrm{Re}=\mathrm{La}, \mathrm{Nd}$ ), can best be visualized as $\mathrm{CuO}_{2}$ planes surrounded by different building blocks. The $\mathrm{Re}_{2} \mathrm{CuO}_{4}$ class of the cuprates can crystallize in three basic forms: the $T$-phase (LSCO), the $T^{\prime}$-phase (NCCO) and a mixed phase, the $T^{*}$-phase. Figure 1.1 shows the general crystal structure for NCCO (a) and LSCO (b). The $\mathrm{Nd}_{2} \mathrm{CuO}_{4} T^{\prime}$-phase has the $\mathrm{CuO}_{2}$ sandwiched between two fluorite type $\mathrm{Nd}_{2} \mathrm{O}_{2}$ layers. The $\mathrm{La}_{2} \mathrm{CuO}_{4} T$-phase can be seen as a $\mathrm{CuO}_{2}$ plane sandwiched between two rock salt type $\mathrm{La}_{2} \mathrm{O}_{2}$ blocks that are mirrored in the $\mathrm{CuO}_{2}$ layer. The main difference between the $T$ and the $T^{\prime}$-phase lies in the respective presence and absence of apical oxygen above and below the $\mathrm{CuO}_{2}$ planes as illustrated by the oxygen octahedron for the LSCO structure. Finally, in the mixed $T^{*}$-phase the $\mathrm{CuO}_{2}$ planes are sandwiched between a $\mathrm{La}_{2} \mathrm{O}_{2}$ block on one side and a $\mathrm{Nd}_{2} \mathrm{O}_{2}$ block on the other side [26]. The $T^{*}$-phase shows all $(00 l)$ 
(a)

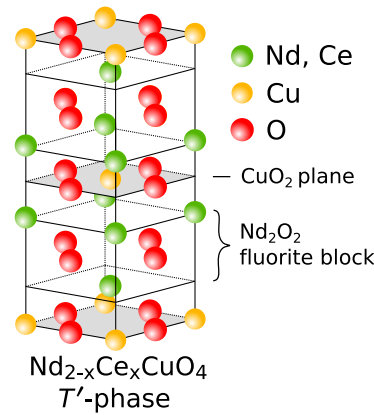

(b)

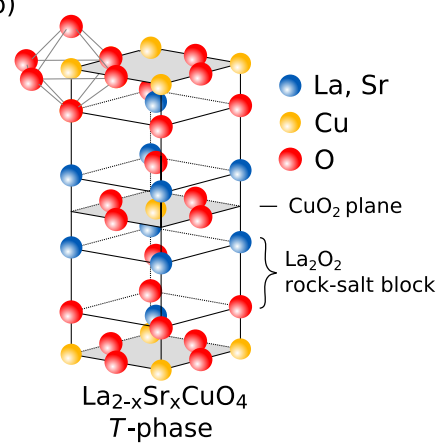

Figure 1.1: Crystal structure for $\mathrm{NCCO}$ (a) and $\mathrm{LSCO}(\mathbf{b})$, the $\mathrm{CuO}_{2}$ planes are indicated by a gray background and the respective fluorite and rock salt cation blocks are labeled. The distinct difference between the $T^{\prime}$-phase and the $T$-phase is the presence of apical oxygen in the latter, indicated by the oxygen octahedron at the top of the crystal structure.

diffraction peaks in X-ray diffraction, whereas the $T$-phase and the $T^{\prime}$-phase only show the peaks for even $l[26]$.

The stability of the different $R_{2} \mathrm{CuO}_{4}$ phases is determined by the tolerance factor $t[27]$ :

$$
t=\frac{r_{i}\left(R e^{3+}\right)+r_{i}\left(O^{2-}\right)}{\sqrt{2}\left[r_{i}\left(C u^{2+}\right)+r_{i}\left(O^{2-}\right)\right]},
$$

where $r_{i}$ is the ionic radius associated with the relevant ions. The tolerance factor $t$ determines the competition between the ionic Re-O bonds and the covalent $\mathrm{Cu}-$ $\mathrm{O}$ bonds. The Re-O bonds have a larger thermal expansion and therefore $t$ will increase with temperature. The $T^{\prime}$-phase will become more stable as $t$ becomes smaller, i.e. as the size of the Re-O bond shrinks. This explains why NCCO with the small ionic radius for $\mathrm{Nd}$ naturally crystallizes into the $T^{\prime}$-phase whereas LSCO prefers the $T$-phase [27]. A material like $\mathrm{La}_{2-\mathrm{x}} \mathrm{Ce}_{\mathrm{x}} \mathrm{CuO}_{4}$ is on the border of the $T^{\prime}$ to $T$ transition and its crystal structure can be tuned by choice of substrate and by doping; higher Ce doping will favor the $T^{\prime}$-phase and isovalent substitution of La by $\mathrm{Y}$ can also stabilize the $T^{\prime}$-phase $[27,28]$.

\subsubsection{Phase diagram}

Strong correlations are a driving force behind the physics of the cuprates. On-site Coulomb repulsion drives the system into a Mott insulator state at half filling, i.e. for the undoped parent compounds. To be more precise, the parent compounds form a charge-transfer insulator (CT) state [30], because the $\mathrm{Cu} 3 d$ lower Hubbard band drops below the $\mathrm{O} 2 p$ band, see figure 1.2(b). Here $U$ is the on-site Coulomb repulsion and $\Delta$ is the resulting charge-transfer gap. The general phase diagram 
(a)

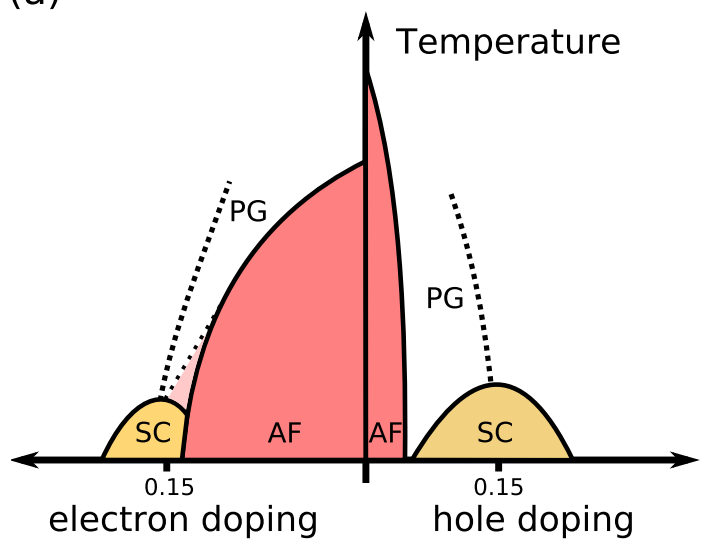

(b)

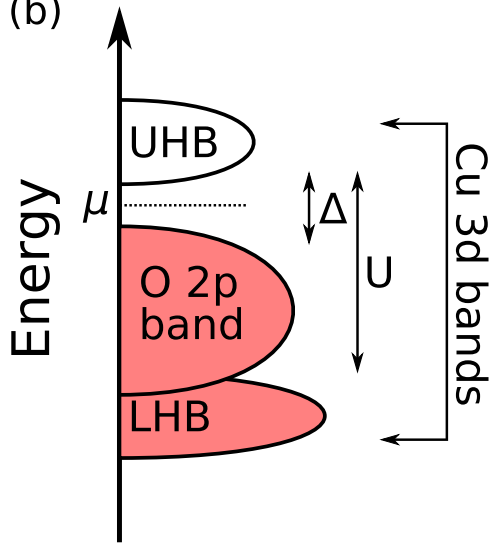

Figure 1.2: (a) Schematic phase diagram of the cuprates (after Armitage et al. [29]). The superconducting (SC), antiferromagnetic (AF) and pseudogap $(\mathrm{PG})$ regions are indicated. The nature of the $\mathrm{PG}$ region is not known exactly and might not be the same on both sides of the phase diagram. Not shown are a spin-glass phase for the $p$-doped cuprates at low temperature between the $\mathrm{AF}$ and $\mathrm{SC}$ region and the strange metal phase that exists beyond the $\mathrm{PG}$ region on the $p$-doped side. The light red area at the $n$-doped side indicates the potential coexistence of the SC and the AF phase. (b) Schematic density of states (DOS) for the charge-transfer insulator state at zero doping. Indicated are the Fermi level $(\mu)$, the upper and lower Hubbard bands, corresponding to the $\mathrm{Cu} 3 d$ states (UHB and LHB, respectively), $U$ is the level splitting of the Hubbard bands, corresponding to the on-site Coulomb energy, and $\Delta$ is the charge transfer energy.

as a function of doping is very similar for electron and hole-doping, see figure 1.2(a), where an initial antiferromagnetic (AF) ordering in the CT state gives way to superconductivity (SC) for higher doping and eventually the superconductivity disappears and we find more or less Fermi-liquid behavior. The difference between electron and hole-doping becomes apparent in the finer details. In general, the AF regime is larger on the $n$-doped side and the critical temperature $\left(T_{c}\right)$ of the superconducting phase is also generally lower. It is not clear whether the AF phase and the SC phase coexist in the $n$-doped case, this is indicated by the light red area [29]. In a simple picture, electrons are doped into the $\mathrm{Cu} 3 d$ upper Hubbard band and holes are doped into the $\mathrm{O} 2 p$ band. Here we ignore any reconstruction and additional spectral weight transfer that does occur. The effect of this doping picture is that on the $p$-doped side the AF lattice is frustrated by additional spins on oxygen sites and on the $n$-doped side it is merely diluted by compensating existing spins on copper sites. This explains why the AF phase extends to higher doping on the $n$-doped side. On the $p$-doped side, the AF quickly disappears upon doping and there is an intermediate spin-glass phase at low temperatures between the AF phase and the SC phase. At higher temperatures both materials show a pseudogap phase, where some gap remains near the Fermi level. The origin of 
the pseudogap is still under debate and it may not have the same origin on either side of the phase diagram [29]. The pseudogap phase will be discussed in some more detail in chapter 6 . Beyond the pseudogap phase on the $p$-doped side, we find a strange metal phase above the superconducting dome that transitions into a normal, Fermi liquid-like phase for higher doping (not shown in the figure).

\subsection{Requirements for combination}

\subsubsection{Annealing procedure and oxygenation}

One major hurdle in the thin film combination of electron and hole-doped cuprates is conflicting oxygenation requirements. For optimal superconducting properties, the electron-doped cuprates are generally annealed in a vacuum after deposition, whereas the hole-doped cuprates are annealed in an oxygen environment. In the case of the hole-doped cuprates, the annealing is required to ensure full oxygenation of the crystal structure, i.e. to ensure that there are no missing apical oxygen atoms and no vacancies in the $\mathrm{CuO}_{2}$ planes.

The role of the reduction step is still under debate, but the general consensus is that a small amount of oxygen is removed during the reduction [31]. The electron doped cuprates are generally reduced at high temperatures in flowing inert gas or vacuum [29]. This reduction step has proved to be necessary for superconductivity $[32,33]$. The reduction is performed at temperatures and oxygen partial pressures approaching or even crossing the stability line of the $R E_{2-\mathrm{x}} \mathrm{Ce}_{\mathrm{x}} \mathrm{CuO}_{4}$ phase and close to the $\mathrm{Cu}_{2} \mathrm{O} / \mathrm{CuO}$ stability line $[34,35]$. It is argued that this is required in order to be able to remove oxygen from the $\mathrm{CuO}_{2}$ planes. However, as a consequence partial decomposition can occur, which is accompanied by the formation of a $\mathrm{Cu}$ poor $(R E, \mathrm{Ce})_{2} \mathrm{O}_{3}$ phase [34,36-38]. Therefore, the optimal reduction is a trade-off between a high $T_{c}$ and partial decomposition. In this thesis, we mainly focus on $\mathrm{NCCO}$ for which we use a reduction procedure involving a $8 \mathrm{~min}$ dwell at $740^{\circ} \mathrm{C}$ in a vacuum of $10^{-5}-10^{-6} \mathrm{mbar}$. Extrapolating the stability diagram of Kim and Gaskell [34], we find that this annealing and part of the subsequent cool down also crosses the stability line for NCCO.

In most cases Ce doping is used as a source of charge carriers and a certain doping level $(\mathrm{x}=0.1-0.18)$ is required to achieve superconductivity [29], but undoped compounds have also been made superconducting simply by reduction [39-41]. In the latter case, superconductivity is achieved by a two-step annealing procedure [41] or by isovalent substitution of $\mathrm{La}$ by $\mathrm{Y}$ in $\mathrm{La}_{2} \mathrm{CuO}_{4}[39,40]$.

There are several proposals for the role of the reduction in the $n$-type cuprates. First of all the removal of oxygen acts as a source of electrons, similar to the effect of Ce substitution $[32,33,42]$. This alone does not explain the appearance of a superconducting phase, since extra Ce doping does not result in superconductivity. We can identify three main mechanisms that have been shown to play a role in the growth of the electron doped cuprates: 
1. The reductions removes excess apical oxygen above and below the $\mathrm{CuO}_{2}$ planes, thereby reducing impurity scattering and disorder [31,43-45].

2. The reduction removes oxygen from the $\mathrm{CuO}_{2}$ planes, breaking the antiferromagnetic order [41,46-49].

3. The reduction restores $\mathrm{Cu}$ vacancies in the $\mathrm{CuO}_{2}$ planes, which is accompanied by the formation of a $\mathrm{Cu}$ poor $(R E, \mathrm{Ce})_{2} \mathrm{O}_{3}$ parasitic phase [37,38,50-52].

Which of these three mechanisms leads to superconductivity, is still under debate [29]. Raman spectroscopy and infrared transmission measurements suggest that the effect of the reduction is even doping dependent; for low doping mainly apical oxygen appears to be removed and near optimal doping, oxygen is mostly removed from the $\mathrm{CuO}_{2}$ planes $[46,47]$. Recent experiments by Krockenberger et al. suggest that the reduction process might be a two step process [41]. The authors claim that a high temperature low oxygen pressure annealing removes oxygen from the crystal $\left(\mathrm{Pr}_{2-\mathrm{x}} \mathrm{Ce}_{\mathrm{x}} \mathrm{CuO}_{4}\right.$ thin films in this case), mainly from the $\mathrm{CuO}_{2}$ planes; a second vacuum annealing at a lower temperature allows apical oxygen that is not removed in the first annealing to fill the vacancies in the $\mathrm{CuO}_{2}$ planes. With a two-step annealing procedure, Krockenberger et al. find a different phase diagram than for the usual high temperature vacuum annealing, with the highest $T_{c}$ at zero doping [41].

\subsubsection{Combinations of electron and hole-doped cuprates}

There are several reports on the combination of electron and hole-doped cuprates in literature, most of which focus on the combination of hole-doped $\mathrm{YBa}_{2} \mathrm{Cu}_{3} \mathrm{O}_{7-\mathrm{x}}$ (YBCO) with electron-doped NCCO [53-57]. Initial experiments have shown that YBCO and $\mathrm{NCCO}$ can be combined on a single chip and that, by either oxygen or vacuum annealing, superconductivity can be switched between the two layers [53]. A specific annealing procedure tailored to the oxygenation requirements of $\mathrm{YBCO}$ and the reduction requirements for $\mathrm{NCCO}$ allows both layers to become superconducting [56]. Other experiments employed $\mathrm{N}_{2} \mathrm{O}$ as a deposition gas for pulsed laser deposition (PLD) instead of $\mathrm{O}_{2}$ for $\mathrm{NCCO}[54,58]$. The $\mathrm{N}_{2} \mathrm{O}$ has a lower dissociation energy $(1.67 \mathrm{eV}[59])$ than $\mathrm{O}_{2}(5.11 \mathrm{eV}[60]) . \quad \mathrm{N}_{2} \mathrm{O}$ is thermally quite stable and relatively unreactive. It will efficiently dissociate to produce atomic oxygen by photon- or electron-impact dissociation. Therefore the oxygen incorporation occurs during the laser pulse and there is low oxygen activity between pulses. This results in a lower overall oxygen concentration and the samples do not require an extra anneal step in vacuum. This was successfully used to first create a superconducting $c$-axis contact between $\mathrm{NCCO}$ and YBCO [54] that was later shown also to be a Josephson contact using three different junction geometries [55]: a supercurrent was shown to flow in a $c$-axis contact using both a directly fabricated contact and a $c$-axis contact via a bicrystal grain boundary, and in an in-plane ramp-edge contact. The latter also had a substantial $c$-axis contact parallel to the in-plane contact. Other, more recent works focus on the combination of the electron-doped $\mathrm{Pr}_{2-\mathrm{x}} \mathrm{Ce}_{\mathrm{x}} \mathrm{CuO}_{4}$ with either YBCO [61] or LSCO [62]. 


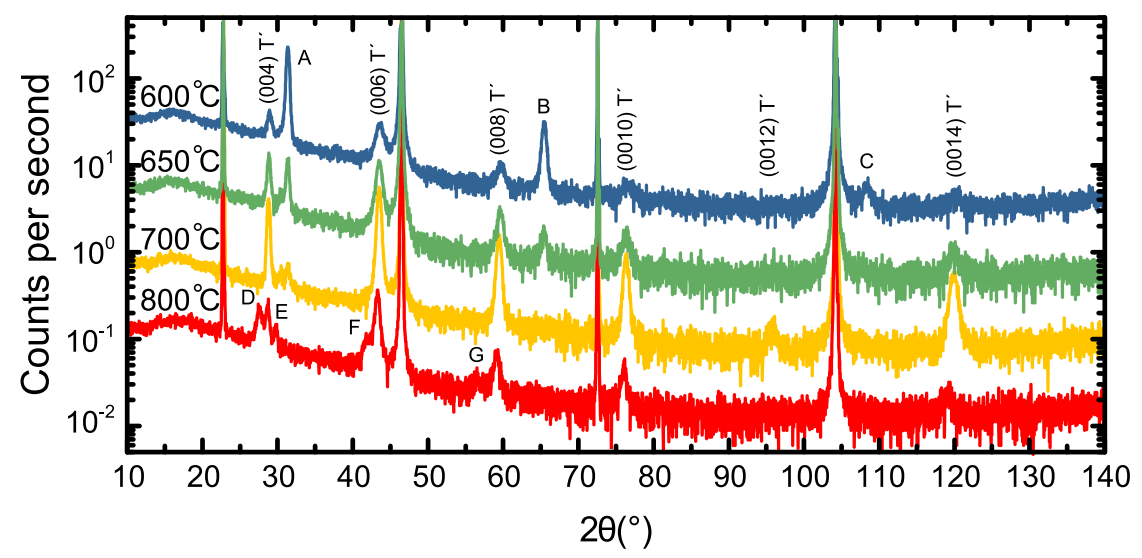

Figure 1.3: XRD $\theta-2 \theta$ spectra for $\mathrm{La}_{2-\mathrm{x}} \mathrm{Ce}_{\mathrm{x}} \mathrm{CuO}_{4}, x=0.11$, as function of deposition temperature. The films are annealed for $15 \mathrm{~min}$ in vacuum after deposition at $650{ }^{\circ} \mathrm{C}$ for the $700{ }^{\circ} \mathrm{C}$ and $800^{\circ} \mathrm{C}$ curves and at $630^{\circ} \mathrm{C}$ and $580^{\circ} \mathrm{C}$ for the $650{ }^{\circ} \mathrm{C}$ and $600^{\circ} \mathrm{C}$ curves, respectively. The LCCO $T^{\prime}$-phase is indicated and other peaks of interest are labeled $\mathrm{A}-\mathrm{G}$, they are discussed in the main text.

\subsubsection{LSCO and NCCO}

For our work we have chosen to pursue a combination of LSCO and NCCO because these are the simplest cuprates with an isolated $\mathrm{CuO}_{2}$ plane and the carrier density can be tuned by either Sr or Ce doping. This in contrast to e.g. YBCO, where most of the carrier tuning is done by oxygen off-stoichiometry. Our intended experiments toward exciton formation and the current quantization work discussed in chapter 6 rely on a control of the doping level and carrier density of the cuprates. Since we have less control over the oxygen stoichiometry in the combination experiment, due to the inherent difference between the electron and hole-doped cuprates, a material like LSCO, with the extra control over the doping level, is preferred over YBCO.

\subsection{4 $\mathrm{La}_{2-\mathrm{x}} \mathrm{Ce}_{\mathrm{x}} \mathrm{CuO}_{4}$}

A more natural combination in terms of elemental compatibility and lattice parameters, would be the combination of LSCO with $\mathrm{La}_{2-\mathrm{x}} \mathrm{Ce}_{\mathrm{x}} \mathrm{CuO}_{4}$ (LCCO), which is also an electron doped cuprate superconductor. LCCO is an interesting material since it can crystallize in both the $T^{\prime}$-phase and the $T$-phase. It can be made superconducting in the $T^{\prime}$-phase by either a combination of vacuum annealing and isovalent substitution of $\mathrm{Y}[39,40]$ or by Ce substitution, where the optimum lies around $x=0.11[63,64]$. The $T$-phase of LCCO has been shown to not become superconducting, but instead shows increasingly stronger insulating behavior upon Ce doping [65]. 
We have explored the growth of LCCO by pulsed laser deposition on $\mathrm{SrTiO}_{3}$ substrates using polycrystalline targets fabricated by solid state reaction. Figure 1.3 shows X-ray diffraction $\theta-2 \theta$ scans for $\mathrm{La}_{1.89} \mathrm{Ce}_{0.11} \mathrm{CuO}_{4}$ films of $50 \mathrm{~nm}$ thickness for different deposition temperatures. For temperatures below $700^{\circ} \mathrm{C}$, the spectra are dominated by a crystal phase other than $T^{\prime}$-LCCO at $31.4^{\circ}, 65.5^{\circ}$ and $108.5^{\circ}$, peaks $\mathrm{A}-\mathrm{C}$, which we attribute to either a misoriented (2 20$)$ oriented LCCO phase or a copper deficient phase isostructural with cubic $\mathrm{La}_{2} \mathrm{O}_{3}[66,67]$. The former being the more likely candidate, since the phase is suppressed for higher deposition temperature, which is also seen in e.g. YBCO with the appearance of $a$ axis oriented YBCO for low deposition temperatures [68]. A copper deficient phase is expected to be promoted at higher temperatures and this phase disappears for higher temperatures. Above $700{ }^{\circ} \mathrm{C}$ the extra peaks seen at the lower temperatures have disappeared, but two new peaks appear; one peak below $T^{\prime}$-LCCO (004) and one peak above. We attribute the first peak to the $T$-phase of LCCO, as this is expected to be more stable at higher temperatures following equation (1.1) [69]. Higher order peaks can also be identified: labels D, F and G indicate the (002), (004) and (006) of the LCCO $T^{\prime}$-phase, respectively. The second peak (E) cannot be exactly identified, since no higher order peaks can be identified. It is presumed to originate from a copper deficient phase with a different crystal structure than the potential $\mathrm{La}_{2} \mathrm{O}_{3}$ parasitic phase found at the lower temperatures. The films grown at $700^{\circ} \mathrm{C}$ show mostly $c$-axis oriented $T^{\prime}$-LCCO with only minor contributions from other phases.

With this experiment we show that, even though from a composition perspective LCCO is a good match for LSCO, it is not suitable for combinations due to the presence of multiple competing phases. Therefore, we have chosen use $\mathrm{Nd}_{2-\mathrm{x}} \mathrm{Ce}_{\mathrm{x}} \mathrm{CuO}_{4}$ instead, because it has a more stable $T^{\prime}$-phase, especially towards under-doping, due to the smaller ionic radius of $\mathrm{Nd}$ compared to La [70].

\subsubsection{Substrate choice}

There are two basic requirements for the substrate to be used in the combination of NCCO and LSCO. The first is a reasonable lattice match with the two materials, and the second is that the substrate has to remain inert and insulating after all the processing steps. Therefore, we have chosen to use $\left[\mathrm{LaAlO}_{3}\right]_{0.3}\left[\mathrm{Sr}_{2} \mathrm{AlTaO}_{6}\right]_{0.7}$ (LSAT), as it has a lattice parameter of $3.87 \AA$ that falls between $3.949 \AA$ for $\mathrm{Nd}_{1.85} \mathrm{Ce}_{0.15} \mathrm{CuO}_{4}$ [38] and $3.775 \AA$ for $\mathrm{La}_{1.85} \mathrm{Sr}_{0.15} \mathrm{CuO}_{4}$ [71], and it remains insulating under vacuum annealing and argon ion milling. LSAT is preferred over the commonly used $\mathrm{SrTiO}_{3}$ (STO), because STO can easily become conducting during processing, in particular during argon ion milling [72-74]. An added benefit is that the Czochralski growth of the LSAT source crystals generally yields a cleaner material with less defects than the flame fusion (Verneuil) growth of STO [75].

Single termination of the surface is not a strict requirement for our experiments, but atomically smooth surfaces are preferred to keep the interfaces sharp and reduce film roughness. The LSAT substrates are annealed for 10 hours at $1050{ }^{\circ} \mathrm{C}$ 


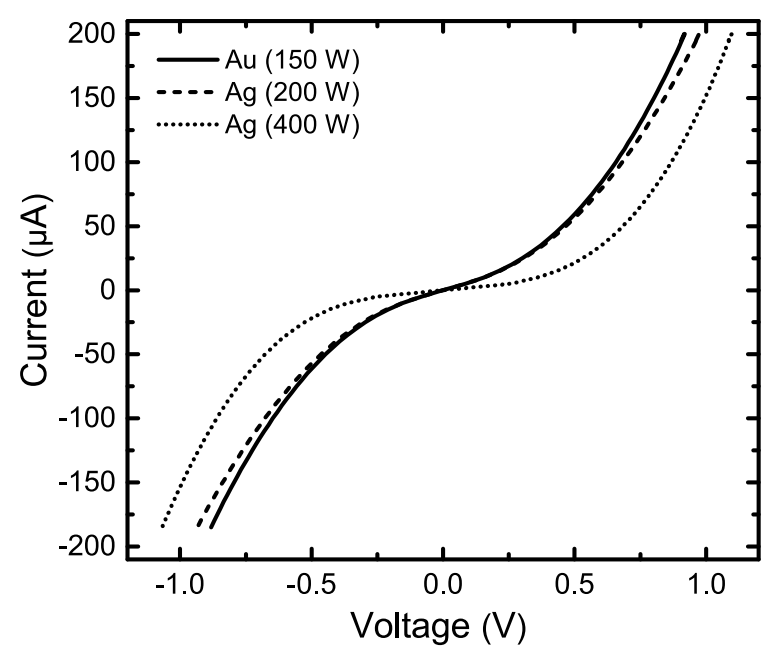

Figure 1.4: Contact resistance $I V$ characteristics for $\mathrm{Au}$ and $\mathrm{Ag}$ contacts to $\mathrm{La}_{1.81} \mathrm{Sr}_{0.09} \mathrm{CuO}_{4}$; two-point measurements on LSCO strips with the strip resistance subtracted. All contact materials are deposited by sputter deposition with $\mathrm{Au}$ at $150 \mathrm{~W}$ (solid line) and $\mathrm{Ag}$ at $200 \mathrm{~W}$ (dashed line) and $400 \mathrm{~W}$ (dotted line).

in flowing oxygen $\left(150 \mathrm{~mL} \mathrm{~min}^{-1}\right)$, which results in an atomically smooth surface with unit cell and half unit cell steps [76,77].

\subsubsection{Contact resistance}

One of the main challenges in measuring our devices has turned out to be contact resistance. The contact resistance of gold to NCCO shows an ohmic behavior, but LSCO has been found to form a Schottky-type contact with contact metals like gold or silver [78] and on e.g. Nb-STO [79]. In semiconductor physics, an ohmic contact between a $p$-type semiconductor and a metal is usually found when the work function of the metal is larger than that of the semiconductor, ignoring effects like Fermi level pinning [80]. The work function of LSCO varies between 5.02 and $5.23 \mathrm{eV}$, depending on doping [79]. There is only a very limited number of metals that have a work function high enough to potentially form an ohmic contact to LSCO, also a common problem with $p$-type semiconductors. From reference data on polycrystalline metals, the only viable candidates are $\mathrm{Au}$ with $5.1 \mathrm{eV}$ and $\mathrm{Pt}$ with $5.65 \mathrm{eV}$ [81]. Low contact resistance has also been reported for $\mathrm{Ag}$ [82,83], although this is not directly expected from its work function of $4.26 \mathrm{eV}$ [81]. Here, the lowered contact resistance can be attributed to Ag diffusion into the LSCO during an annealing step [83]. We have explored sputter deposited $\mathrm{Au}$ and $\mathrm{Ag}$ contact for LSCO, since we did not have Pt readily available. Figure 1.4 shows twopoint $I V$ measurements on $\mathrm{La}_{1.91} \mathrm{Sr}_{0.09} \mathrm{CuO}_{4}$ strips with $200 \mu \mathrm{m} \times 200 \mu \mathrm{m}$ contact pads of $\mathrm{Au}$ and $\mathrm{Ag}$ where the strip resistance has been subtracted as derived 
from a separate four-point measurement. For each of the contact materials, this measurement is done for strips varying in length from $50 \mu \mathrm{m}$ to $500 \mu \mathrm{m}$ to verify the subtraction method. As contact materials we use Au, deposited at $150 \mathrm{~W}$ and $\mathrm{Ag}$, deposited at $200 \mathrm{~W}$ and $400 \mathrm{~W}$. Both Au and Ag layers are $200 \mathrm{~nm}$ thick. For the Ag, the higher power level is expected to yield a better adhesion of the layer and may mimic the diffusion effect employed by others [83]. In figure 1.4 we can see that this is not the case. The lowest contact resistance for the $\mathrm{Ag}$ is found using the lower deposition power. The contact resistance is very similar to what we find for $\mathrm{Au}$, with the $\mathrm{Au}$ contact resistance being slightly lower. This is what is expected from the work functions of the two materials and is also observed in other experiments [78]. Furthermore, we have found that gold has a higher adhesion to both NCCO and LSCO than silver, making fabrication easier. Especially for smaller devices, sonication to facilitate the lift-off of photoresist will remove large parts of the silver contact, whereas gold contacts remain unaffected. In many of our experiments we use the common procedure of sputter depositing a nanometer thin $\mathrm{Ti}$ adhesion layer before depositing the gold. Titanium has a work function of $4.33 \mathrm{eV}$ [81], which might influence the contact resistance in a negative way. In reality the titanium has only a minor influence due to the small thickness of the layer.

In this thesis we discuss in-plane contacts (chapters 3 and 6) and $c$-axis contacts (chapter 5). The contact resistance is a more important contribution to bilayer and multilayer devices, especially for a $c$-axis contact. Here, the contact size to the top layer is limited by the junction size and is a purely $c$-axis contact, without any in-plane contribution. In general this will give a higher resistance than for example in the in-plane contacts described in chapter 3, where the LSCO-gold contact can be extended over a larger area, also incorporating $a b$-plane contact along the edges.

In semiconductor technology, ohmic contacts from metallic electrodes to a semiconductor are created by over-doping the semiconductor under the metallic contacts. This lowers the contact resistance by limiting the size of the depletion layer. A similar approach might be used in the contact to optimally doped LSCO; an extra layer of over-doped LSCO can be used to mediate the contact. This layer would have to be heavily overdoped, since the work function of LSCO peaks around $x=0.2$ [79]. We expect the depletion region to be smaller for the metallic, overdoped LSCO. In our experiments, we have chosen to not yet implement this step to avoid overcomplication and because this would add another $p n$-interface, which might obscure the properties of the actual $p n$-interface between NCCO and LSCO. For the in-plane $p n$-junctions this is less of an issue, as here the voltages probes can be placed so only the interface of interest is measured. For the perpendicular contacts described in this chapter that can only be realized by adding an extra etching step to define the extra over-doped layer into separated contacts before depositing the metallic layer. 


\subsection{Semiconductor model for LSCO/NCCO junctions}

We employ band alignment theory from semiconductor physics in order to estimate the important energy scales in $p n$-junction between $\mathrm{La}_{1.85} \mathrm{Sr}_{0.15} \mathrm{CuO}_{4}$ and $\mathrm{Nd}_{1.85} \mathrm{Ce}_{0.15} \mathrm{CuO}_{4}$. Here, we use two different approaches: the first is an Anderson/Schottky-Mott type band alignment, where the work function $\phi$ of both materials determines the band alignment; the second approach assumes chemical bonding and hybridization at the interface, in this case a hybridization of the oxygen $2 p$ orbitals of the valence band that extend in-plane, which might be the case for in-plane junctions. In this scenario, the valence band in the LSCO would line up with the valence band in the NCCO, since both bands originate from oxygen $2 p$ states, see also figure $1.2(\mathrm{~b})$.

\subsubsection{General band diagram}

A comparative band diagram of $\mathrm{La}_{2} \mathrm{CuO}_{4}$ and $\mathrm{Nd}_{2} \mathrm{CuO}_{4}$ has been constructed by Yunoki et al. [84], see figure 1.5. From this we get an estimate of the size of the band gap for both $\mathrm{Nd}_{2} \mathrm{CuO}_{4}$ and $\mathrm{La}_{2} \mathrm{CuO}_{4}$ and the position of the Fermi level with respect to the bottom of the valence band.

The chemical potential shift as a function of doping for both materials has been determined from core level shifts by Harima et al. [85]. Li et al. [79] have measured the chemical potential shift in LSCO using LSCO/Nb:STO junctions. From the core level shifts, the chemical potential of LSCO stays nearly constant up to optimal doping [85], but in the heterojunctions a shift of around $0.1 \mathrm{eV}$ is observed for optimal doping [79]. For NCCO, there is a continuous shift, increasing up to $0.2 \mathrm{eV}$ for a doping level of $x=0.15$ [85].

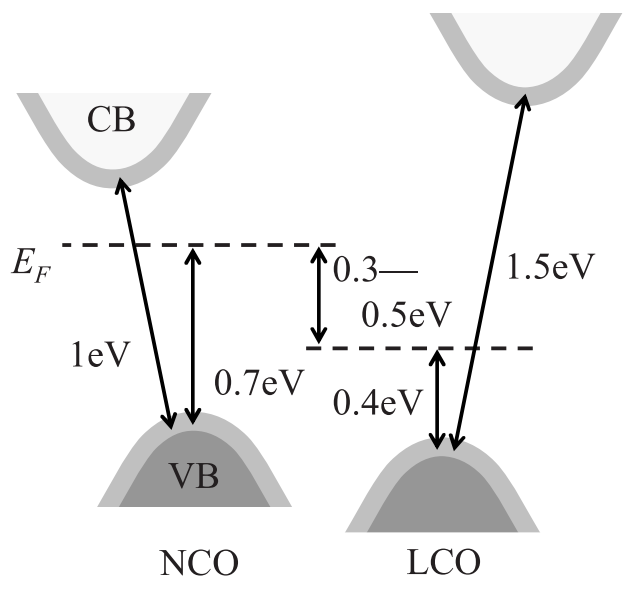

Figure 1.5: Schematic band diagram for $\mathrm{Nd}_{2} \mathrm{CuO}_{4}$ and $\mathrm{La}_{2} \mathrm{CuO}_{4}$ (from Yunoki et al. [84]). 


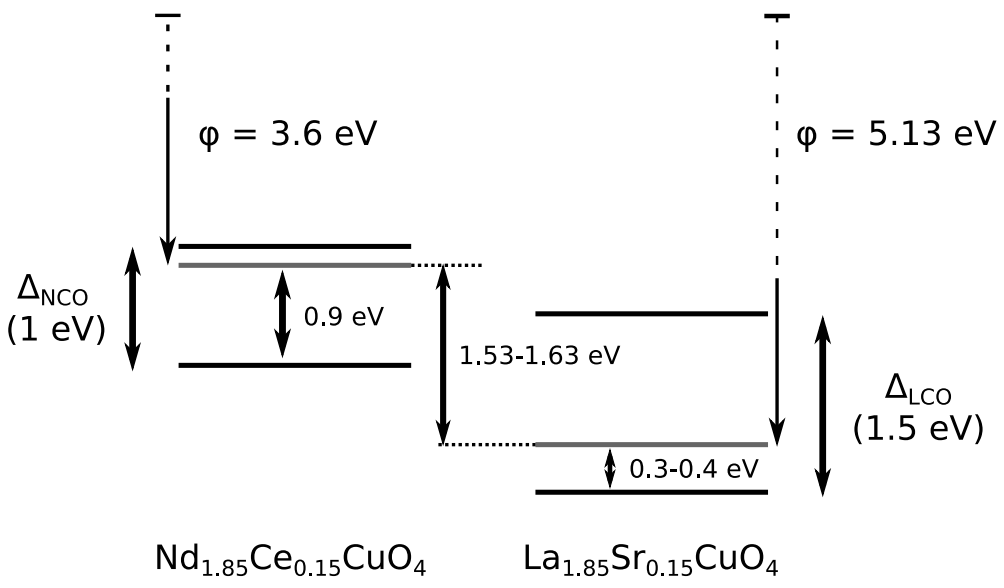

Figure 1.6: Anderson/Schottky-Mott type band alignment for NCCO-LSCO.

\subsubsection{Anderson/Schottky-Mott band alignment}

For an Anderson/Schottky-Mott type band alignment, the bands align according to the work function of the materials and the Fermi level mismatch is determined by the difference in work function. Therefore, knowledge of the work functions of the materials in question is required [80]. The work function for $\mathrm{La}_{2-\mathrm{x}} \mathrm{Sr}_{\mathrm{x}} \mathrm{CuO}_{4}$ varies with the doping level and has been determined from heterojunctions with Nb-doped $\mathrm{SrTiO}_{3}$ [79]. For $\mathrm{La}_{1.85} \mathrm{Sr}_{0.15} \mathrm{CuO}_{4}$, a work function of $5.13 \mathrm{eV}$ can be deduced from these measurements. No detailed study has been done on the work function of $\mathrm{Nd}_{2-\mathrm{x}} \mathrm{Ce}_{\mathrm{x}} \mathrm{CuO}_{4}$. From ARPES measurements, we can get a sample specific work function of $3.6 \mathrm{eV}$ as quoted by Sakisaka et al. for $\mathrm{Nd}_{2-\mathrm{x}} \mathrm{Ce}_{\mathrm{x}} \mathrm{CuO}_{4}$ with $x \sim 0.17$ [86].

Figure 1.6 shows the band alignment using the work functions determined for $\mathrm{Nd}_{1.85} \mathrm{Ce}_{0.15} \mathrm{CuO}_{4}$ and $\mathrm{La}_{1.85} \mathrm{Sr}_{0.15} \mathrm{CuO}_{4}$. Here, we take the work function for $\mathrm{Nd}_{1.85} \mathrm{Ce}_{0.15} \mathrm{CuO}_{4}$ to be the same as that reported by Sakisaka et al. [86]. The band alignment is close to a type III broken gap configuration and we find a Fermi energy difference of $1.53-1.63 \mathrm{eV}$. This band alignment is comparable to what Charlebois et al. use to model the PCCO-LSCO interface [12]. Mannhart et al. also assume a Fermi level difference of several volts, resulting in a similar band alignment picture that leads to their prediction of Esaki diode behavior [10].

\subsubsection{Valence band hybridization}

When we consider a contact between NCCO and LSCO, it is reasonable to assume there will be overlap of the oxygen orbitals in the $\mathrm{CuO}_{2}$ planes, especially for an inplane contact. The oxygen $2 p$ orbitals make up the valence band and these can then be expected to hybridize. When we assume valence band hybridization, we arrive at a picture very similar to the band alignment proposed by Yunoki et al. [84], see 


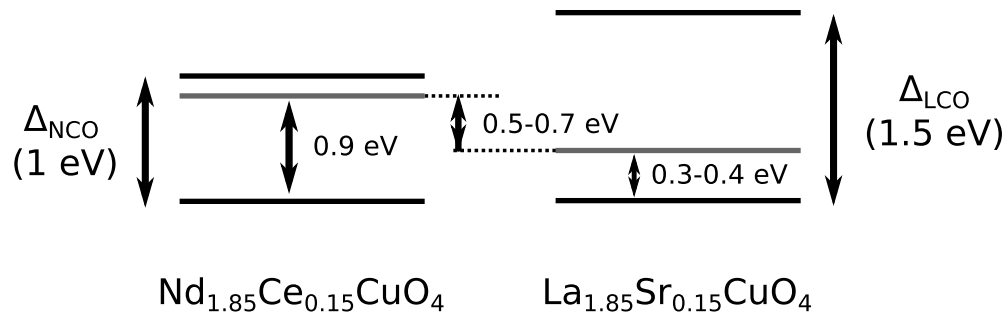

Figure 1.7: Schematic band alignment assuming valence band hybridization.

figure 1.7. When the valence band of both undoped parent compounds is taken at the same level, the Fermi level jump is $0.3 \mathrm{eV}$. The Fermi level jump going from an electron doped compound to a hole doped compound has been reported as high as $0.5 \mathrm{eV}[84,87]$. Therefore we use the $0.3-0.5 \mathrm{eV}$ range shown in the figure by Yunoki et al. [84] as a starting point. We then find a Fermi level difference of 0.5-0.7 eV for the optimally doped NCCO-LSCO contact, taking into account a Fermi level shift of $0.2 \mathrm{eV}$ for $\mathrm{Nd}_{1.85} \mathrm{Ce}_{0.15} \mathrm{CuO}_{4}$, compared to the undoped case. This leads to a type I straddling gap configuration.

\subsubsection{Band bending}

Sticking to the semiconductor model, we can now sketch what happens to the bands when the Fermi levels equilibrate in a contact between NCCO and LSCO. Figure 1.8 shows a possible result for the band alignments discussed in $\$ 1.3 .2$ (a) and $\S 1.3 .3(\mathrm{~b})$. For the top panels of figure 1.8 , we have made one more assumption, that we will also use later in chapter 3: we assume that most of the band bending occurs for the NCCO. From the phase diagram discussed in $\S 1.1 .2$ (figure 1.2), we know that the AF phase associated with the Mott insulator ground state extends much further for the electron doped cuprates. Therefore, NCCO should be described as a degenerate semiconductor whereas LSCO should already behave more like a metal. In figure 1.8(a), this assumption leads to the appearance of hole-like interface states, an effect also predicted by Charlebois et al. [12]. Band bending in the LSCO will move the cusp leading to the interface states to lower energy. These states will drop below the Fermi level when the band bending is approximately equal on both sides of the junction, see the bottom panel of figure 1.8(a). For the valence band hybridization scenario the band bending is more continuous than in the Anderson/Schottky-Mott case, leading to no unusual features at the interface.

\subsubsection{Thomas-Fermi screening length}

An important parameter for electronic interactions is the Thomas-Fermi screening length $\lambda_{T F}$, which determines the length over which electronic interactions still play a role $[88,89]$. It can be used to get a order of magnitude estimate for the 
(a)
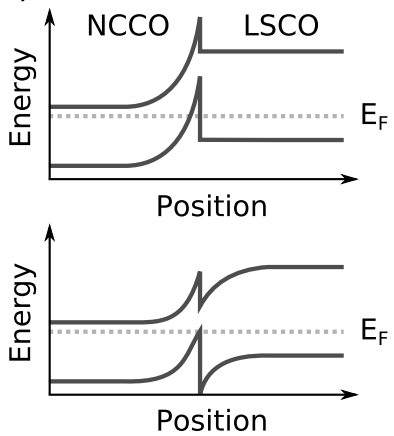

Anderson/Schottky-Mott (b)

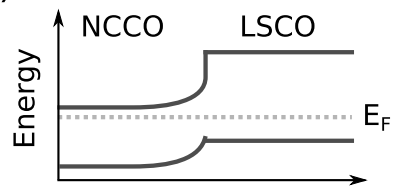

Position

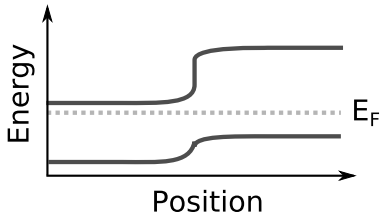

Valence band hybridization

Figure 1.8: Band bending at the LSCO/NCCO interface. The top panels assume only band bending on the NCCO side of the junction, the bottom panels assume band bending in both materials. (a) Band bending for the Anderson/Schottky-Mott band alignment of $\S 1.3 .2$. Interface states appear for this scenario, as also suggested by Charlebois et al. [12]. (b) Band bending for the valence band hybridization scenario of $\S 1.3 .3$.

length scale of the band bending effects discussed in the previous sections. In general, $\lambda_{T F}$ is very small in metals and can be quite large, up to micrometers, in semiconductors. The cuprates are somewhere between metals and semiconductors in terms of carrier density, so $\lambda_{T F}$ is expected to be quite small. However, this is compensated by the cuprates (any many other oxides) having a large relative permittivity [90], which increases $\lambda_{T F}$. The Thomas-Fermi screening length is given by $[88,89]$ :

$$
\lambda_{T F}=\sqrt{\frac{\epsilon \epsilon_{0}}{4 \pi e^{2} \partial n / \partial \mu}},
$$

where $\epsilon_{0}$ is the vacuum dielectric constant, $\epsilon$ is the relative permittivity and $\partial n / \partial \mu$ is the density of states at the Fermi level. For typical cuprates the screening length is found to be of the order of the dimensions of the unit cell, $\sim 4 \AA[91$, 92]. Equation (1.2) assumes an isotropic, three-dimensional system. This is not completely valid for the cuprates that show a pronounced anisotropy with most of the conductivity limited to the $\mathrm{CuO}_{2}$ planes. LSCO in particular is know for its layered two dimensional nature [93,94]. It is therefore expected that $\lambda_{T F}$ is also anisotropic in the cuprates. Ahn et al. [92] have derived an estimate for $\lambda_{T F}$ by using Poisson's equation to describe the response of an idealized layered system of charged sheets to an applied potential. Perpendicular to the sheets this leads to a $\lambda_{T F}$ that depends on the layer spacing, but when the carrier density is factored in, this leads to the same equation as equation (1.2). Since we find $\lambda_{T F}$ to be of the order of the lattice spacing, the use of Poisson's equation is debatable here, as the continuity approximation breaks down. For the in-plane $\lambda_{T F}$, equation (1.2) seems more applicable, but here also $\lambda_{T F}$ approaches the inter-particle distance. 
Taking this into account, we can argue that the screening length will be larger than the $\lambda_{T F}$ estimate of equation (1.2), due to inefficient screening in both the out-of-plane direction because of the discrete layer spacing, and in the in-plane direction because of to the two-dimensional nature of the conducting layers and the continuity approximation breakdown.

\subsubsection{Depletion zone}

We can use a continuum approximation to get an estimate for the depletion zone in our junctions for the two different band alignments we have discussed. This approach would be more valid for an in-plane junction than for an out-of-plane junction, since in-plane, the continuum approximation might still be valid. For a contact between two semiconductors, Poisson's equation can be used to estimate the depletion zone. We can write down the Poisson's equation for everywhere in a general $p n$-device, where we only consider the $x$-direction that crosses the $p n$-interface:

$$
\frac{\mathrm{d}^{2} \phi}{\mathrm{d} x^{2}}=-\frac{e}{\epsilon \epsilon_{0}}\left(p-n+N_{D}-N_{A}\right),
$$

where $\phi(x)$ is the potential, $\epsilon_{0}$ is the vacuum dielectric constant, $\epsilon$ is the relative permittivity, $p(n)$ is the local hole (electron) concentration and $N_{D}\left(N_{A}\right)$ is the concentration of donors (acceptors). This equation can be used to estimate the width of the depletion region at the $p n$-interface. Here we assume that the electric field vanishes outside of the depletion region and that there are no charges due to free carriers inside the depletion region. Within the depletion zone, equation (1.3) reduces to:

$$
\frac{\mathrm{d}^{2} \phi}{\mathrm{d} x^{2}}= \begin{cases}-\frac{e N_{D}}{\epsilon \epsilon_{0}} & \text { for } 0 \leq x \leq l_{n} \\ \frac{e N_{A}}{\epsilon \epsilon_{0}} & \text { for }-l_{p} \leq x \leq 0\end{cases}
$$

where $l_{n}$ and $l_{p}$ represent the depletion zone on the $n$-doped and the $p$-doped side, respectively. Next we impose the boundary conditions $\phi^{\prime}\left(-l_{p}\right)=0, \phi^{\prime}\left(l_{n}\right)=0$, $\phi\left(-l_{p}\right)=\phi_{p 0}$ and $\phi\left(l_{n}\right)=\phi_{n 0}$. Equation (1.4) becomes:

$$
\frac{\mathrm{d}^{2} \phi}{\mathrm{d} x^{2}}= \begin{cases}-\frac{e N_{D}}{2 \epsilon \epsilon_{0}}\left(x-l_{n}\right)^{2}+\phi_{n 0} & \text { for } 0 \leq x \leq l_{n} \\ \frac{e N_{A}}{2 \epsilon \epsilon_{0}}\left(x+l_{p}\right)^{2}+\phi_{p 0} & \text { for }-l_{p} \leq x \leq 0 .\end{cases}
$$

Demanding continuity of the potential at $x=0$, we get:

$$
\left.\phi\right|_{x \uparrow 0}-\left.\phi\right|_{x \downarrow 0}=\phi_{p o}-\phi_{n 0}+\frac{e N_{A}}{2 \epsilon \epsilon_{0}} l_{p}^{2}+\frac{e N_{D}}{2 \epsilon \epsilon_{0}} l_{n}^{2}=0 .
$$


In this equation we can substitute the built-in potential $\left(V_{b i}=\phi_{n 0}-\phi_{p 0}\right)$ and eliminate $l_{p}$ by invoking charge neutrality $\left(N_{A} l_{p}=N_{D} l_{n}\right)$ :

$$
V_{b i}=\frac{e N_{D}}{2 \epsilon \epsilon_{0} N_{A}}\left(N_{A}+N_{D}\right) l_{n}^{2}
$$

From this we can get an expression for $l_{n, p}$ :

$$
l_{n, p}=\left(\frac{2 \epsilon \epsilon_{0} N_{A, D} V_{b i}}{e N_{D, A}\left(N_{A}+N_{D}\right)}\right)^{1 / 2} .
$$

For the NCCO-LSCO $p n$-junction, we can simplify further by taking $N_{A}=N_{D}=$ $N$. This give a depletion width:

$$
W=2\left(\frac{\epsilon \epsilon_{0} V_{b i}}{e N}\right)^{1 / 2}
$$

With equation (1.9) we can estimate the size of the depletion zone in a $p n$-junction between $\mathrm{Nd}_{1.85} \mathrm{Ce}_{0.15} \mathrm{CuO}_{4}$ and $\mathrm{La}_{1.85} \mathrm{Sr}_{0.15} \mathrm{CuO}_{4}$. As an approximation we can take both cuprates as degenerate semiconductors with the same carrier density $N \sim 1-5 \times 10^{21} \mathrm{~cm}^{-3}[10]$; the relative permittivity is 20-40 for the cuprates [90]; and the built-in voltage can be estimated from the band diagrams discussed in the previous sections, see figures 1.6 and 1.7. In an Anderson/Schottky-Mott type band alignment, the built-in voltage is determined by the difference in work function between the two materials, $1.5-1.6 \mathrm{eV}$, this gives a depletion zone of 1 $4 \mathrm{~nm}$. In the valence band hybridization scenario, the valence bands of NCCO and LSCO would align and the built-in voltage corresponds to the Fermi level difference found to be $0.5-0.7 \mathrm{~V}$. With this, we find a depletion zone of $0.5-2.5 \mathrm{~nm}$.

So far we have assumed similar depletion on both sides. For a depletion zone dominated by the NCCO electrode with negligible depletion in the LSCO layer, the depletion layer becomes a factor $\sqrt{2}$ smaller.

\subsection{Conclusions}

In this chapter we have discussed the relevant properties of the cuprates NCCO and LSCO and we have identified oxygenation as the main conflicting parameter in a combination experiment. In the next chapter we study the effect of the oxygenation procedure used for LSCO on the properties of NCCO.

Using a semiconductor approach, we have estimated the size and energy scale associated with a depletion zone at the $p n$-interface between LSCO and NCCO. This will be tested experimentally in an in-plane ramp-edge configuration in chapter 3 and in a $c$-axis configuration in chapter 5 . 



\section{Effect of high oxygen pressure annealing on $\mathrm{Nd}_{1.85} \mathrm{Ce}_{0.15} \mathrm{CuO}_{4}$}

The quality of $\mathrm{Nd}_{1.85} \mathrm{Ce}_{0.15} \mathrm{CuO}_{4}$ films grown by pulsed laser deposition is shown to be enhanced by using a non-stoichiometric target with extra copper added to suppress the formation of a parasitic $(\mathrm{Nd}, \mathrm{Ce})_{2} \mathrm{O}_{3}$ phase. The properties of these films are less dependent on the exact annealing procedure after deposition as compared to films grown from a stoichiometric target. Film growth can be followed by a 1 bar oxygen annealing, after an initial vacuum annealing, while retaining the superconducting properties and quality. This enables the integration of electron-doped cuprates with their hole-doped counterparts on a single chip, to create, for example, superconducting $p n$-junctions.

\subsection{Introduction}

As noted in the previous chapter, with the substitutional doping in cuprates by Ce in electron-doped $\mathrm{Nd}_{2-\mathrm{x}} \mathrm{Ce}_{\mathrm{x}} \mathrm{CuO}_{4}$ and $\mathrm{Sr}$ in hole-doped $\mathrm{La}_{2-\mathrm{x}} \mathrm{Sr}_{\mathrm{x}} \mathrm{CuO}_{4}$, the parallel to semiconductors is easy to make. Indeed, various theoretical analyses have been made on the properties and possibilities of combinations of electron- and holedoped cuprates, ranging from the formation of a Mott insulator depletion zone [12], unconventional Josephson junctions [10,11] and superradiant light emission [95]. Oxygen plays an important role in the realization of combinations of electron- and hole-doped cuprates. The role of oxygen in $n$-type cuprates is widely researched and debated $[29,37,41,43,45-48,96]$. The consensus is that an oxygen reduction is necessary for superconductivity and that strong oxygenation leads to loss of superconductivity and increasing resistance. However, the exact mechanism for oxygen

Part of this chapter was published as: M. Hoek et al., Effect of high oxygen pressure annealing on superconducting $\mathrm{Nd}_{1.85} \mathrm{Ce}_{0.15} \mathrm{CuO}_{4}$ thin films by pusled laser deposition from Cu-enriched targets, Superconductor Science and Technology 27044017 (2014). 
reduction is still under debate, in particular where oxygen is removed from the unit cell and what the consequences are for the structure of the cuprate [37,41,45,48,97]. The necessity of a reduction is in stark contrast to the oxygenation needed for optimal superconductivity in the $p$-type cuprates: in general, $p$-type cuprates require an oxygenation step at high oxygen pressures. These conflicting annealing requirements are one of the main hurdles to the realization of combinations of electron- and hole-doped cuprates.

In this chapter we investigate the effect of strong oxygenation that is needed for the growth of a $p$-type cuprate like $\mathrm{La}_{2-\mathrm{x}} \mathrm{Sr}_{\mathrm{x}} \mathrm{CuO}_{4}$, on the $n$-type cuprate $\mathrm{Nd}_{2-\mathrm{x}} \mathrm{Ce}_{\mathrm{x}} \mathrm{CuO}_{4}$. Growth of $R E_{2-\mathrm{x}} \mathrm{Ce}_{\mathrm{x}} \mathrm{CuO}_{4}(R E=\mathrm{Nd}, \mathrm{Pr}, \ldots)$ as single crystals grown by e.g. the traveling-solvent floating-zone technique $[36-38,52]$ or as thin films by pulsed laser deposition (PLD) [29,97-99] is almost always accompanied by the formation of a parasitic phase of $(R E, C e)_{2} \mathrm{O}_{3}$, due to copper deficiency during growth and during oxygen reduction. Other techniques like molecular beam epitaxy [100] and dc sputtering [101] appear to be less sensitive to the formation of the $(R E, C e)_{2} \mathrm{O}_{3}$ phase. For PLD, it has been shown that this parasitic phase can be suppressed in $\mathrm{Pr}_{2-\mathrm{x}} \mathrm{Ce}_{\mathrm{x}} \mathrm{CuO}_{4}$ by adding extra copper to the PLD target [97]. We show that the parasitic $(\mathrm{Nd}, \mathrm{Ce})_{2} \mathrm{O}_{3}$ phase in $\mathrm{Nd}_{1.85} \mathrm{Ce}_{0.15} \mathrm{CuO}_{4}$ can also be suppressed by using copper-rich targets and that these films retain their quality and superconducting properties when subjected to oxygen annealing procedures suitable for the growth of $p$-type cuprates.

\subsection{Experimental details}

We compare films grown by PLD using two different targets with effective compositions $\mathrm{Nd}_{1.85} \mathrm{Ce}_{0.15} \mathrm{CuO}_{4}(\mathrm{NCCO})$ and $\mathrm{Nd}_{1.85} \mathrm{Ce}_{0.15} \mathrm{Cu}_{1.1} \mathrm{O}_{4}(\mathrm{NCCO}+)$, the latter containing $10 \%$ extra copper. The targets are prepared by solid state synthesis and the crystal structure is verified by X-ray powder diffraction ${ }^{\dagger}$. The films are deposited at a heater temperature of $820^{\circ} \mathrm{C}$ in 0.25 mbar oxygen using a $\mathrm{KrF}$ excimer laser with a fluency of $1.2 \mathrm{~J} \mathrm{~cm}^{-2}$, a spot size of $5.7 \mathrm{~mm}^{2}$ and a repetition rate of $4 \mathrm{~Hz}$. All films are grown on $(001)$ oriented $\left[\mathrm{LaAlO}_{3}\right]_{0.3}\left[\mathrm{Sr}_{2} \mathrm{AlTaO}_{6}\right]_{0.7}$ (LSAT) substrates that have been annealed in flowing oxygen for 10 hours at $1050{ }^{\circ} \mathrm{C}$ to obtain an atomically smooth surface, as measured by atomic force microscopy (AFM). We have grown films varying in thickness between 30 and $500 \mathrm{~nm}$, with most of the films being $70 \mathrm{~nm}$. We have chosen this value with future device fabrication requirements in mind, although with this thickness the lattice strain from the substrate slightly suppresses $T_{c}[99]$.

We have investigated three different annealing procedures for the $\mathrm{Nd}_{1.85} \mathrm{Ce}_{0.15} \mathrm{CuO}_{4}$ films, see table 2.1. The first is a standard vacuum annealing where the film is cooled down from $820^{\circ} \mathrm{C}$ at deposition pressure and then annealed in vacuum for $8 \mathrm{~min}$ at $740{ }^{\circ} \mathrm{C}$ followed by a cool down in vacuum at $10^{\circ} \mathrm{C} \mathrm{min}^{-1}$ (procedure

$\dagger$ The targets were fabricated by X. Renshaw Wang at the National University of Singapore (NUS). 
Table 2.1: Overview of the three different annealing procedures, standard, long and oxygen. All procedures start with a cool down in deposition pressure from $820^{\circ} \mathrm{C}$ to $740{ }^{\circ} \mathrm{C}$ and all temperature changes are performed at $10^{\circ} \mathrm{C} \mathrm{min}{ }^{-1}$. Cool down II is performed in vacuum and Oxygen annealing and Cool down III in 1 bar oxygen.

\begin{tabular}{lllll}
\hline Procedure & Vacuum annealing & Cool down II & Oxygen annealing & Cool down III \\
\hline standard & 8 min at $740{ }^{\circ} \mathrm{C}$ & $740{ }^{\circ} \mathrm{C}$ to $\mathrm{RT}^{\dagger}$ & - & - \\
long & $45 \mathrm{~min}$ at $740{ }^{\circ} \mathrm{C}$ & $740{ }^{\circ} \mathrm{C}$ to RT & - & - \\
oxygen & 8 min at $740{ }^{\circ} \mathrm{C}$ & $740-600^{\circ} \mathrm{C}$ & 15 min at $600{ }^{\circ} \mathrm{C}$, & $450{ }^{\circ} \mathrm{C}$ to $\mathrm{RT}$ \\
& & & 30 min at $450{ }^{\circ} \mathrm{C}$ & \\
\hline
\end{tabular}

$\dagger$ Room temperature.

standard in table 2.1). The other two procedures are two opposite cases, to explore the stability of the $\mathrm{Nd}_{1.85} \mathrm{Ce}_{0.15} \mathrm{CuO}_{4}$ films under long reduction and strong oxygenation, respectively (procedures long and oxygen in table 2.1). For the long reduction, the dwell time at $740{ }^{\circ} \mathrm{C}$ is extended to $45 \mathrm{~min}$. For the strong oxygenation procedure there is an initial $8 \mathrm{~min}$ vacuum annealing at $740{ }^{\circ} \mathrm{C}$ and then at $600{ }^{\circ} \mathrm{C}$ the conditions are changed to a common recipe we employ for $p$ doped superconductors like $\mathrm{La}_{2-\mathrm{x}} \mathrm{Sr}_{\mathrm{x}} \mathrm{CuO}_{4}$ and $\mathrm{YBa}_{2} \mathrm{Cu}_{3} \mathrm{O}_{7-\mathrm{x}}$. Here, the film is first annealed in 1 bar oxygen for $15 \mathrm{~min}$ at $600^{\circ} \mathrm{C}$ and then for $30 \mathrm{~min}$ at $450{ }^{\circ} \mathrm{C}$ and subsequently cooled down further in 1 bar oxygen.

The film thickness and quality is investigated by X-ray diffraction (XRD), crosssectional transmission electron microscopy (TEM) and AFM. The thickness is measured by AFM using an edge fabricated by a hard mask lift-off of amorphous $\mathrm{YBa}_{2} \mathrm{Cu}_{3} \mathrm{O}_{7-\mathrm{x}}$ (lift-off using $1 \% \mathrm{H}_{3} \mathrm{PO}_{4}$ ) or gold (lift-off using a KI solution). XRD shows that all films are c-axis oriented with a c-axis length of $12.08(1) \AA$, this is also what is observed for optimally doped single crystal [38]. The films are mostly relaxed for a thickness of $70 \mathrm{~nm}$, as can be seen from figure 2.1(a), which shows the relation between LSAT (213) and NCCO (219) for a $70 \mathrm{~nm}$ NCCO film grown from the target with extra copper added. The reciprocal space map shows that the NCCO film has relaxed on the LSAT substrate, unlike what is observed for other deposition techniques [101]. We measure an RMS surface roughness with AFM on a scan area of $2 \mu \mathrm{m} \times 2 \mu \mathrm{m}$ of $3 \mathrm{~nm}$ for the $\mathrm{Nd}_{1.85} \mathrm{Ce}_{0.15} \mathrm{CuO}_{4}$ films grown using the target with extra copper and $1.5 \mathrm{~nm}$ for the $\mathrm{Nd}_{1.85} \mathrm{Ce}_{0.15} \mathrm{CuO}_{4}$ films from the stoichiometric target, both for films of $70 \mathrm{~nm}$ thickness.

The samples are contacted at the corners by $\mathrm{Al}$ bond wires on $\mathrm{Au} / \mathrm{Ti}$ contact pads in a standard van der Pauw geometry for sheet resistance and Hall measurements in a Quantum Design 9 T PPMS system. Hall measurements are performed up to $4 \mathrm{~T}$ and for low temperatures up to $9 \mathrm{~T}$. The sheet resistance is measured both during both warm up and cool down at a rate varying between 0.1 and $3{ }^{\circ} \mathrm{Cmin}^{-1}$. 
(a) In plane - $\mathrm{a}_{[100]}(\AA)$

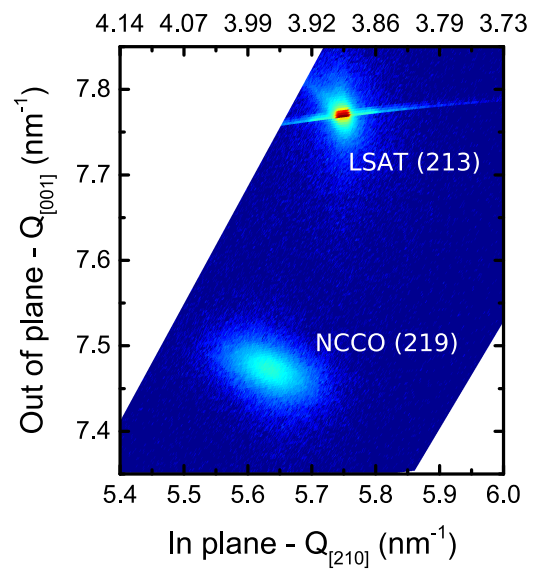

(b)

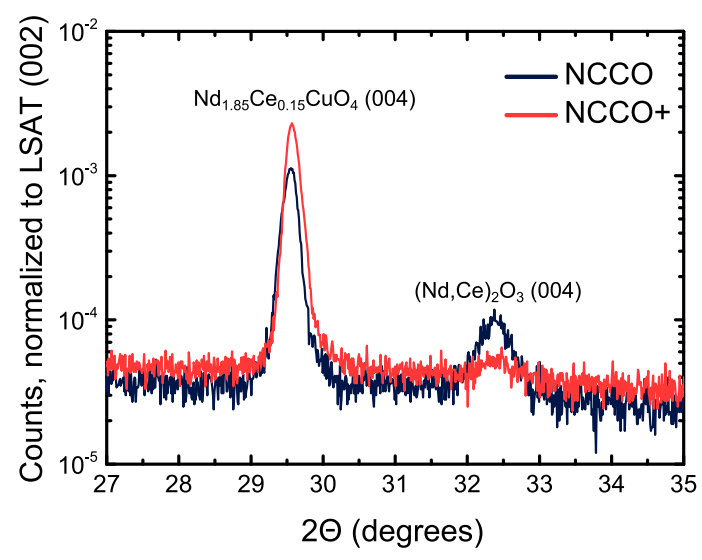

Figure 2.1: (a) Reciprocal space map for an $\mathrm{NCCO}+$ film showing the relation between LSAT (213) and NCCO (219), the axis labels correspond to the NCCO crystal structure. (b) Detail of X-ray diffraction $\theta-2 \theta$ scans normalized to the LSAT (002) peak (not shown in figure) on $70 \mathrm{~nm} \mathrm{Nd}_{1.85} \mathrm{Ce}_{0.15} \mathrm{CuO}_{4}$ films deposited from the stoichiometric target (NCCO, blue) and the nonstoichiometric target with extra copper added $(\mathrm{NCCO}+$, red), showing the suppression of the parasitic $(\mathrm{Nd}, \mathrm{Ce})_{2} \mathrm{O}_{3}$ phase. Both samples have been annealed for $8 \mathrm{~min}$ in vacuum at $740{ }^{\circ} \mathrm{C}$ (standard procedure).

\subsection{Results and discussion}

\subsubsection{Suppression of the $(\mathrm{Nd}, \mathrm{Ce})_{2} \mathrm{O}_{3}$ parasitic phase}

This section compares films grown using the targets with and without extra copper added. Figures 2.1(b), 2.2 and 2.3 compare the same two samples, representative for the general behavior we have observed; both are $70 \mathrm{~nm}$ thick and are annealed in vacuum for $8 \mathrm{~min}$ at $740^{\circ} \mathrm{C}$ after deposition. Figure 2.1(b) shows a detail of the XRD $\theta-2 \theta$ spectra for the two $\mathrm{Nd}_{1.85} \mathrm{Ce}_{0.15} \mathrm{CuO}_{4}$ films, grown with and without extra copper in the target, showing the $\mathrm{Nd}_{1.85} \mathrm{Ce}_{0.15} \mathrm{CuO}_{4}(004)$ and the $(\mathrm{Nd}, \mathrm{Ce})_{2} \mathrm{O}_{3}(004)$ reflection. The spectra are normalized to the $(002)$ Bragg reflection of LSAT (not shown in the figure). With the addition of extra copper to the target, we see a reduction of nearly an order of magnitude in the ratio between the intensities of the $(\mathrm{Nd}, \mathrm{Ce}){ }_{2} \mathrm{O}_{3}(004)$ diffraction peak and the $\mathrm{Nd}_{1.85} \mathrm{Ce}_{0.15} \mathrm{CuO}_{4}$ (0 04 ) peak. Together with this reduction, we observe a higher intensity for the $\mathrm{Nd}_{1.85} \mathrm{Ce}_{0.15} \mathrm{CuO}_{4}(004)$ peak, while the full width at half maximum is slightly decreased (from $0.23^{\circ}$ to $0.19^{\circ}$ ), indicating a higher crystallinity for the films grown 

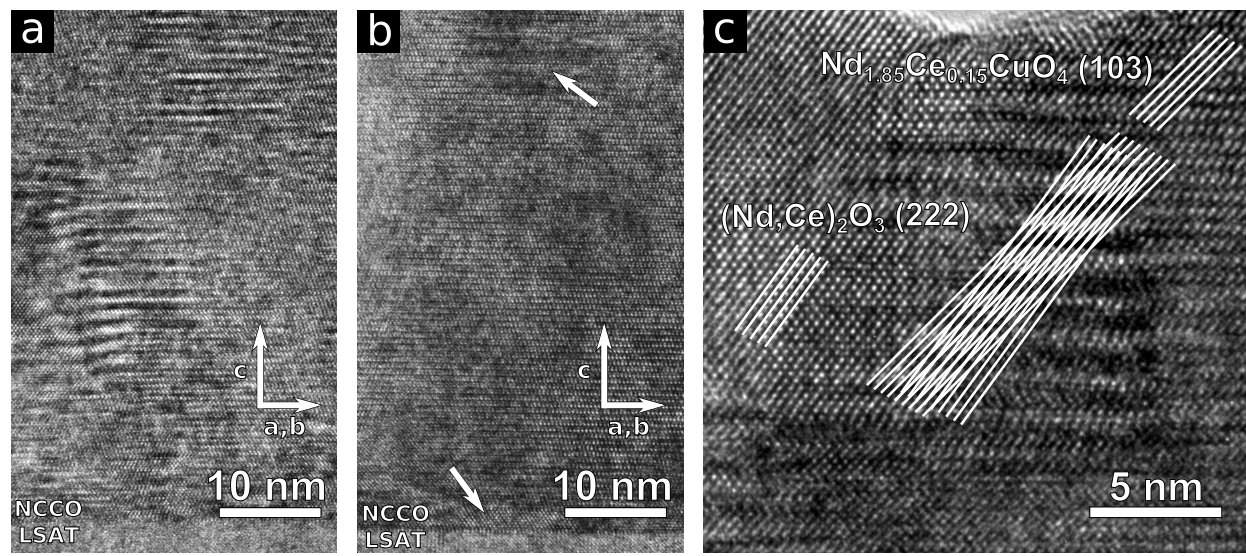

Figure 2.2: Transmission electron microscopy close-ups near the substrate of $70 \mathrm{~nm} \mathrm{Nd} \mathrm{Nd}_{1.85} \mathrm{Ce}_{0.15} \mathrm{CuO}_{4}$ films deposited from the stoichiometric target (a) and the non-stoichiometric target with extra copper added (b); both films are annealed following the standard procedure, see table 2.1. (a) $\mathrm{Nd}_{1.85} \mathrm{Ce}_{0.15} \mathrm{CuO}_{4}$ from the stoichiometric target shows Moiré fringes, caused by the inclusion of $(\mathrm{Nd}, \mathrm{Ce})_{2} \mathrm{O}_{3}$ in the NCCO lattice. (b) $\mathrm{Nd}_{1.85} \mathrm{Ce}_{0.15} \mathrm{CuO}_{4}$ from the nonstoichiometric target with extra copper shows higher $\mathrm{Nd}_{1.85} \mathrm{Ce}_{0.15} \mathrm{CuO}_{4}$ phase purity with only a small inclusion of the parasitic phase, indicated by the white arrows at the top and the bottom of the figure. (c) Close-up of a (Nd,Ce) ${ }_{2} \mathrm{O}_{3}$ inclusion showing the construction of the Moiré pattern by the overlap of the $\mathrm{Nd}_{1.85} \mathrm{Ce}_{0.15} \mathrm{CuO}_{4}$ (103) lattice planes and the (Nd,Ce) ${ }_{2} \mathrm{O}_{3}(222)$ lattice planes, as indicated by the white lines.

with extra copper ${ }^{\dagger}$. The $(\mathrm{Nd}, \mathrm{Ce})_{2} \mathrm{O}_{3}$ phase is still not fully suppressed, suggesting that an even higher percentage of copper may be required for full suppression. We find no appreciable difference in the c-axis lattice parameter between the different annealing procedures for films grown with either of the two targets.

Figure 2.2 shows TEM close-ups of two $\mathrm{Nd}_{1.85} \mathrm{Ce}_{0.15} \mathrm{CuO}_{4}$ films near the substrate/film interface, grown using the two targets without, figure $2.2(\mathrm{a})$, and with extra copper, figure $2.2(\mathrm{~b})$, both annealed in vacuum for $8 \mathrm{~min}$ at $740{ }^{\circ} \mathrm{C}$. For the film grown using the stoichiometric target, the presence of the parasitic $(\mathrm{Nd}, \mathrm{Ce})_{2} \mathrm{O}_{3}$ phase is confirmed by the appearance of Moiré patterns in the TEM image. The image shows the crystal planes of $\mathrm{Nd}_{1.85} \mathrm{Ce}_{0.15} \mathrm{CuO}_{4}$ and areas

$\dagger$ This is mainly inferred from the peak intensity. The FWHM in a $\theta-2 \theta$ spectrum for a thin film is mostly dominated by finite thickness broadening. We can take the FWHM measured for the NCCO (004) peaks in figure 2.1(b) and use the Scherrer formula [102] for crystallite size analysis to get an estimate for the thickness:

$$
S=\frac{k \lambda}{W \cos \theta},
$$

where $k$ is a shape factor (between 0.8 and 1.2), $\lambda$ is the wavelength, $\theta$ is the angle of the peak and $W$ is the FWHM in radians. Taking the shape factor to be 1 , we get a size of around 50 $\mathrm{nm}$, of the same order as the film thickness. 
with a different periodicity; here, a Moiré pattern is formed by an overlap of the $\mathrm{Nd}_{1.85} \mathrm{Ce}_{0.15} \mathrm{CuO}_{4}$ lattice with the lattice of the $(\mathrm{Nd}, \mathrm{Ce})_{2} \mathrm{O}_{3}$ parasitic phase, namely the $\mathrm{Nd}_{1.85} \mathrm{Ce}_{0.15} \mathrm{CuO}_{4}$ (103) lattice planes and the (Nd,Ce) $)_{2} \mathrm{O}_{3}(222)$ lattice planes. The (103) and the (222) planes are sketched in figure 2.2(a), as well as a set of overlapping planes on top of one of the Moiré patterns. The planes are not arbitrary; $\mathrm{Nd}_{1.85} \mathrm{Ce}_{0.15} \mathrm{CuO}_{4}(103)$ and ( $\left.\mathrm{Nd}, \mathrm{Ce}\right)_{2} \mathrm{O}_{3}(222)$ are also the planes that would give the strongest signal in X-ray powder diffraction. The periodicity of the Moiré pattern $\left(d_{M}\right)$ can be calculated from the difference between the g-vectors of the individual lattices [103],

$$
d_{M}=\frac{d_{(103)} d_{(222)}}{\sqrt{d_{(103)}^{2}+d_{(222)}^{2}-2 d_{(103)} d_{(22)} \cos \beta}},
$$

where $d_{(103)}$ and $d_{(222)}$ are the lattice spacings of the $\mathrm{Nd}_{1.85} \mathrm{Ce}_{0.15} \mathrm{CuO}_{4}(103)$ planes and the $(\mathrm{Nd}, \mathrm{Ce})_{2} \mathrm{O}_{3}(222)$ planes, respectively, and $\beta$ is the angle in radians between the $\mathrm{g}$-vectors, normal to these planes. Using the lattice parameters for $\mathrm{Nd}_{1.85} \mathrm{Ce}_{0.15} \mathrm{CuO}_{4}$ and $(\mathrm{Nd}, \mathrm{Ce})_{2} \mathrm{O}_{3}$ from Kimura et al. $[38]\left(d_{(103)}=2.82 \AA\right.$, $d_{(222)}=3.22 \AA$ and $\left.\beta=0.16 \mathrm{rad}\right)$, we find a Moiré pattern spacing of $14.4 \AA$, which is also what we observe in the TEM images.

With the addition of extra copper to the target, we observe a significantly lower density of the parasitic phase in the film. Figure 2.2(b) is a typical example, showing a higher $\mathrm{Nd}_{1.85} \mathrm{Ce}_{0.15} \mathrm{CuO}_{4}$ phase purity with only a small inclusion of the parasitic phase at the substrate interface and at the top of the image (indicated with white arrows). As was also observed in XRD, see figure 2.1(b), the parasitic phase is strongly, but not completely, suppressed.

Figure 2.3 compares the transport properties of the $\mathrm{Nd}_{1.85} \mathrm{Ce}_{0.15} \mathrm{CuO}_{4}$ films. The suppression of the parasitic $(\mathrm{Nd}, \mathrm{Ce})_{2} \mathrm{O}_{3}$ phase is accompanied by in an increase of $T_{c}$ by $3.5 \mathrm{~K}$ to a value of $16.7 \mathrm{~K}\left(T_{c 0}\right)$ for films grown using the non-stoichiometric target, see figure 2.3(a). These films also show a sharper transition to the superconducting phase and a lower normal-state resistance. The sharper transition can be explained by a more homogeneous crystal structure for the films grown from the target with extra copper. The higher resistance for the $\mathrm{Nd}_{1.85} \mathrm{Ce}_{0.15} \mathrm{CuO}_{4}$ films grown from the stoichiometric target may be explained by the $(\mathrm{Nd}, \mathrm{Ce})_{2} \mathrm{O}_{3}$ phase deforming nearby $\mathrm{CuO}_{2}$ planes and acting as nucleation sites for defects and dislocations, all increasing scattering.

The Hall coefficient for both films shows a trend generally observed for $n$-type cuprates around optimal doping, with an upturn towards positive values with decreasing temperature, indicating contribution of hole-like carriers [29, 104-106], see figure 2.3(b). For $\mathrm{Nd}_{1.85} \mathrm{Ce}_{0.15} \mathrm{CuO}_{4}$ films grown from the target with extra copper, the minimum in the Hall coefficient shifts to a higher temperature. For low temperatures, a complete cross-over to a positive Hall coefficient is observed with a cross-over region where the Hall resistance displays both electron and hole-like character, as shown in the inset to figure 2.3(b). The data in figure 2.3(b) only use a low field linear fit to the Hall data, illustrated by the black linear fit in the inset. The difference between the two curves can be explained by the two-band 
(a)

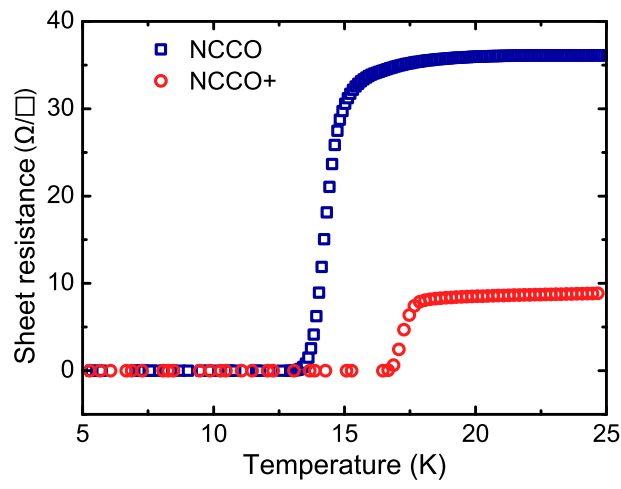

(b)

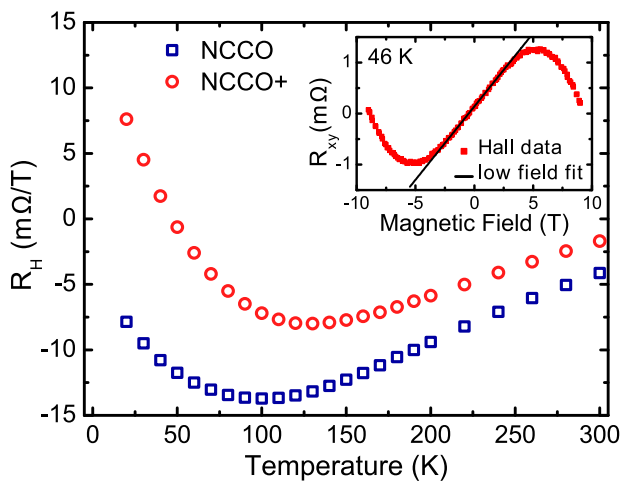

Figure 2.3: Transport measurements on $70 \mathrm{~nm} \mathrm{Nd}{ }_{1.85} \mathrm{Ce}_{0.15} \mathrm{CuO}_{4}$ films deposited from the stoichiometric target (NCCO, blue squares) and the nonstoichiometric target with extra copper added (NCCO+, red circles); both films are annealed in vacuum for $8 \mathrm{~min}$ at $740{ }^{\circ} \mathrm{C}$ (standard procedure). (a) Sheet resistance around $T_{c}$, showing a higher $T_{c}$, sharper transition and lower resistance for the $\mathrm{Nd}_{1.85} \mathrm{Ce}_{0.15} \mathrm{CuO}_{4}$ film with extra copper. (b) Hall coefficient versus temperature measured in the van der Pauw geometry; both films show a pronounced minimum and the $\mathrm{Nd}_{1.85} \mathrm{Ce}_{0.15} \mathrm{CuO}_{4}$ grown from the target with extra copper shows a cross-over to a positive Hall coefficient for low temperature. The inset shows the two-band nature of the Hall resistance at $46 \mathrm{~K}$ and a low field linear fit.

nature of $\mathrm{Nd}_{2-\mathrm{x}} \mathrm{Ce}_{\mathrm{x}} \mathrm{CuO}_{4}$ around optimal doping. Here the Hall coefficient not only measures the carrier density, but a combination of the density and mobility of both carrier types. As we argue that the suppression of the $(\mathrm{Nd}, \mathrm{Ce})_{2} \mathrm{O}_{3}$ phase decreases scattering, this will lead to a higher mobility, which will in turn be reflected in the Hall coefficient.

\subsubsection{Effect of different annealing procedures}

Finally, we look at the effect of different annealing procedures for films grown using the stoichiometric target and the target with extra copper added. The three annealing procedures are as described earlier: $8 \mathrm{~min}$ vacuum (standard), $45 \mathrm{~min}$ vacuum (long), and $8 \mathrm{~min}$ vacuum followed by 1 bar oxygen at $600{ }^{\circ} \mathrm{C}(15 \mathrm{~min})$ and $450{ }^{\circ} \mathrm{C}(30 \mathrm{~min})$ (oxygen), see also table 2.1 . The sheet resistance for all films across the whole temperature range $(2-300 \mathrm{~K})$ is shown in figure $2.4(\mathrm{a})$. The films grown from the target with extra copper show a close grouping of the curves, whereas the films grown with the stoichiometric target show a large spread. The largest deviation is found for the $\mathrm{Nd}_{1.85} \mathrm{Ce}_{0.15} \mathrm{CuO}_{4}$ films grown using the stoichiometric target and annealed in oxygen after the initial vacuum annealing (oxygen procedure). Here, an upturn in the resistance is observed above $T_{c}$, indicating a shift to lower doping by oxygen inclusion [45], increased impurity scattering and carrier 

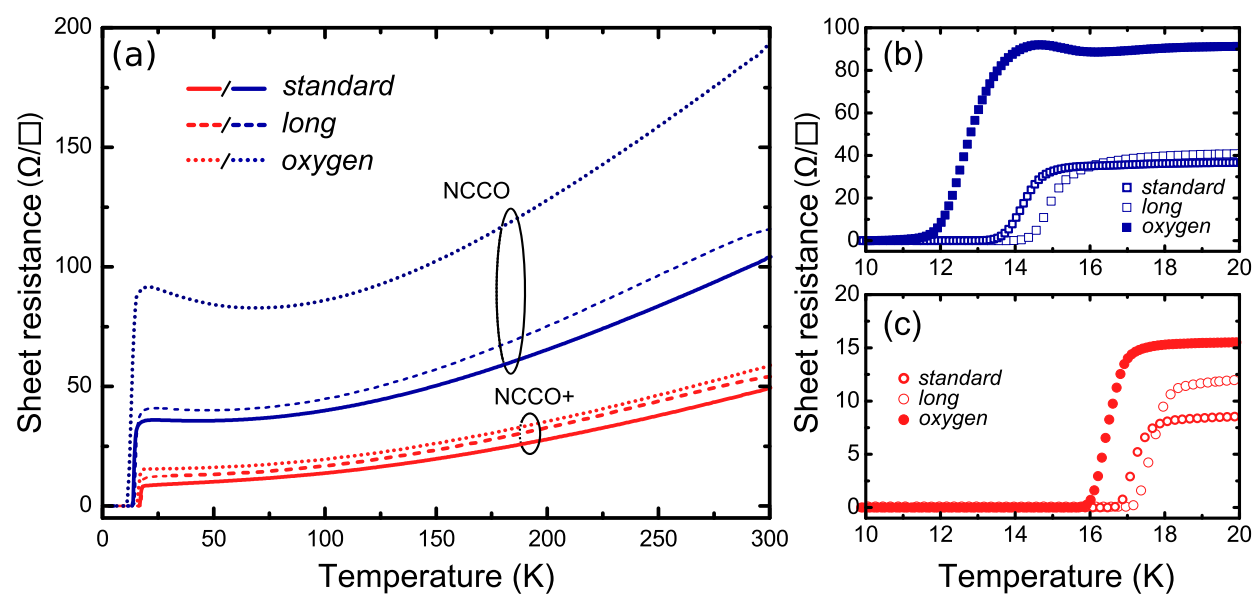

Figure 2.4: (a) Transport measurements on $70 \mathrm{~nm} \mathrm{Nd}_{1.85} \mathrm{Ce}_{0.15} \mathrm{CuO}_{4}$ films deposited from the stoichiometric target (NCCO, blue) and the nonstoichiometric target with extra copper added $(\mathrm{NCCO}+$, red) for three different annealing procedures as described in table 2.1: standard (solid lines), long (dashed lines) and oxygen (dotted lines). (b) Close-up around $T_{c}$ for the $\mathrm{Nd}_{1.85} \mathrm{Ce}_{0.15} \mathrm{CuO}_{4}$ films from the stoichiometric target. (c) Close-up around $T_{c}$ for the $\mathrm{Nd}_{1.85} \mathrm{Ce}_{0.15} \mathrm{CuO}_{4}$ films with extra copper added to the target.

localization [44,105]. These samples show a broad superconducting transition with a small upturn at $T_{c}$, characteristic of sheet measurements on inhomogeneous superconductors with an out-of-line contact arrangement [107], see figure 2.4(b). We find the highest $T_{c}$ for films annealed for $45 \mathrm{~min}$ in vacuum.

The films grown with the target with extra copper show only a $2 \mathrm{~K}$ spread in $T_{c}$ with the different annealing procedures and the width of the superconducting transition is always smaller than $1 \mathrm{~K}$. The same trend as for the films without extra copper is observed, with a 45 min vacuum annealing (long procedure) giving the highest $T_{c}$ and a 1 bar oxygen annealing at $600^{\circ} \mathrm{C}$ (oxygen procedure) the lowest $T_{c}$, see figure 2.4(c). All films with extra copper show a higher $T_{c}$ than the films grown with the stoichiometric target.

The Hall effect measurements confirm the observations from the sheet resistance measurements, see figure $2.5(\mathrm{a}, \mathrm{b})$. The $\mathrm{Nd}_{1.85} \mathrm{Ce}_{0.15} \mathrm{CuO}_{4}$ films grown with the standard target show a wide spread in the Hall coefficient, especially at low temperatures, where the characteristic minimum has completely disappeared for the films annealed following the oxygen procedure, see figure 2.5(a). This was also reported for oxygenated films of $\mathrm{Nd}_{2-\mathrm{x}} \mathrm{Ce}_{\mathrm{x}} \mathrm{CuO}_{4}$ [105] and $\mathrm{Pr}_{2-\mathrm{x}} \mathrm{Ce}_{\mathrm{x}} \mathrm{CuO}_{4}$ [48]. We have observed the same trend for films grown from stoichiometric targets of different doping levels, see appendix 2.4. For the $\mathrm{Nd}_{1.85} \mathrm{Ce}_{0.15} \mathrm{CuO}_{4}$ films with extra copper we see a closer grouping of all the curves and they all show the characteristic minimum, see figure 2.5(b). It is interesting to note that both a longer vacuum annealing and an oxygen annealing following a short vacuum annealing suppresses the cross-over to a positive Hall coefficient, not depending on the presence of 
(a)

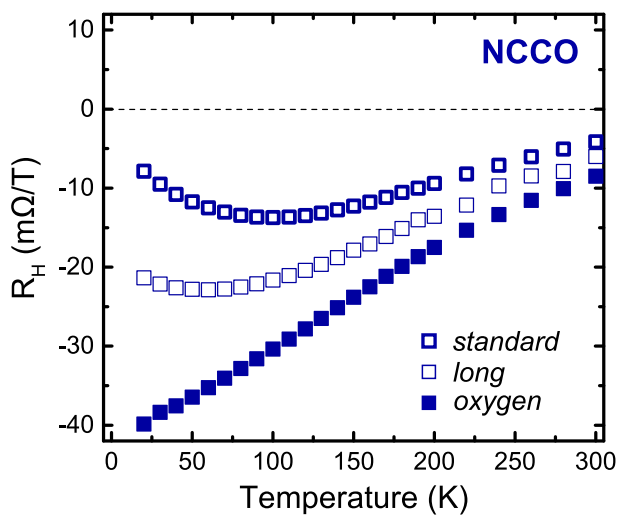

(b)

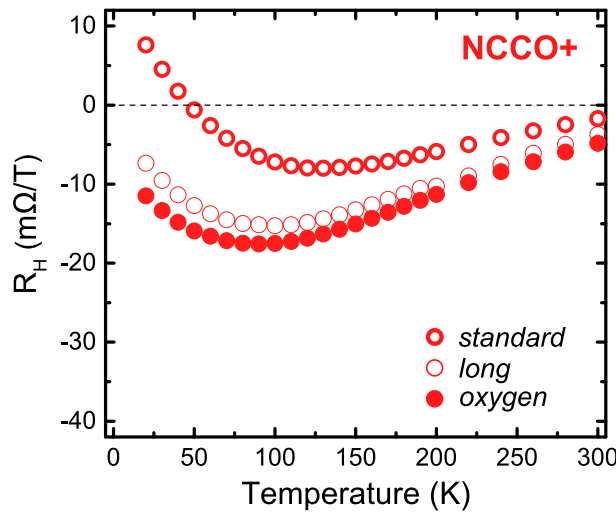

Figure 2.5: Hall measurements on $70 \mathrm{~nm} \mathrm{Nd}_{1.85} \mathrm{Ce}_{0.15} \mathrm{CuO}_{4}$ films deposited from the stoichiometric target (a) and the non-stoichiometric target with extra copper added (b) for the three different annealing procedures as described in table 2.1: standard (half filled symbols), long (open symbols) and oxygen (closed symbols).

the parasitic $(\mathrm{Nd}, \mathrm{Ce})_{2} \mathrm{O}_{3}$ phase. In the latter case this can be explained by the counter-doping effect of oxygen, effectively shifting the doping away from optimal doping, towards underdoping [45]. The former case is not immediately obvious. The cross-over to positive Hall coefficients for the extra copper films with an 8 min vacuum annealing, suggests that the samples are already at optimal doping or even overdoped. It has been shown that, during vacuum annealing, oxygen is removed from the $\mathrm{CuO}_{2}$ planes, suppressing anti-ferromagnetic ordering and promoting hole-like carriers [41,46-49]. This is accompanied by an increased normal-state resistance for over-reduction $[105,108,109]$, attributed to increased defect and impurity scattering [49]. Annealing for 45 min should therefore only shift the Hall coefficient towards more hole-like character, as is commonly observed for oxygen reduction in $\mathrm{Nd}_{2-\mathrm{x}} \mathrm{Ce}_{\mathrm{x}} \mathrm{CuO}_{4}[44,105,110]$. This is not what we observe: we do see an increased normal-state resistance, but the Hall coefficients shifts toward more negative values. We suggest that as more and more oxygen is removed from the $\mathrm{CuO}_{2}$ planes, scattering is increased. As the Hall coefficient for a two-band system is also linked to the mobility of the carriers, our observations can be explained by the scattering centers having more influence on the mobility of the hole-like carriers than on the electron-like carriers. This would explain both the higher normal state resistivity and the shift to a more negative Hall coefficient. 


\subsection{Conclusions}

Our experiments show that the addition of extra copper to the PLD target of $\mathrm{Nd}_{1.85} \mathrm{Ce}_{0.15} \mathrm{CuO}_{4}$ can increase the quality and $T_{c}$ of thin films by suppressing the formation of the parasitic $(\mathrm{Nd}, \mathrm{Ce})_{2} \mathrm{O}_{3}$ phase. The presence or absence of the parasitic $(\mathrm{Nd}, \mathrm{Ce}){ }_{2} \mathrm{O}_{3}$ phase in $\mathrm{Nd}_{1.85} \mathrm{Ce}_{0.15} \mathrm{CuO}_{4}$ films has a strong influence on $T_{c}$ and the transport properties above $T_{c}$. For long reduction the difference is minimal, while the biggest difference is observed for strong oxygenation. The relatively minor influence of the exact annealing procedure on $T_{c}$ and the Hall coefficient for $\mathrm{Nd}_{1.85} \mathrm{Ce}_{0.15} \mathrm{CuO}_{4}$ films grown with extra copper in the target suggests that studies into the exact role of oxygen in the reduction process for $n$-type cuprates should not overlook the influence the presence of the $(R E, \mathrm{Ce})_{2} \mathrm{O}_{3}$ parasitic phase can have. We have also shown that the $\mathrm{Nd}_{1.85} \mathrm{Ce}_{0.15} \mathrm{CuO}_{4}$ films with extra copper can even be subjected to a 1 bar oxygen annealing suitable for the growth of $p$-type cuprates such as $\mathrm{La}_{2-\mathrm{x}} \mathrm{Sr}_{\mathrm{x}} \mathrm{CuO}_{4}$ and $\mathrm{YBa}_{2} \mathrm{Cu}_{3} \mathrm{O}_{7-\mathrm{x}}$. This makes $\mathrm{Nd}_{2-\mathrm{x}} \mathrm{Ce}_{\mathrm{x}} \mathrm{CuO}_{4}$ grown from a non-stoichiometric target with extra copper a prime candidate for studying $p n$-physics in the cuprate superconductors, opening the way to integrate electron-doped cuprates with their hole-doped counterparts on a single chip. Such combinations are of interest for example for the creation of superconducting $p n-$ junctions or to explore electron-hole interactions in the rich phase diagram of the cuprates.

\section{Appendices}

\section{A Effect of oxygen annealing for different doping levels}

We have explored the phase diagram for $\mathrm{Nd}_{2-\mathrm{x}} \mathrm{Ce}_{\mathrm{x}} \mathrm{CuO}_{4}$ without extra copper added and we find the highest $T_{c}$ for the target with the intended doping of $x=0.15$ (out a set of targets for $x=0.1,0.135,0.14,0.15,0.18$ and 0.2 ). The composition of the targets is inferred from the ratios of the starting powders in the target fabrication and from X-ray diffraction, we have done no direct measurements of the Ce-content of the films and the targets. Figure A.1 shows the effect on the Hall coefficient of the standard and oxygen annealing procedures described in table 2.1 for a selection of films with different doping levels grown on $\mathrm{SrTiO}_{3}$. The figure compares additional measurements on films with $x=0.2$ and $x=0.135$ doping to the $x=0.15$ films described in the main text of this chapter. The open symbols represent the standard vacuum annealing and illustrate the change of the Hall coefficient with doping level. For $x=0.135$ (open circles) the Hall coefficient decreases continuously; for the two $x=0.15$ films we observe an upturn towards positive values at low temperatures with a cross-over for the film grown from the target with extra copper added; and for $x=0.2$ the Hall coefficient is positive over the whole temperature range. This matches with what is commonly observed for the $n$-doped cuprates $[29,104,106]$. 


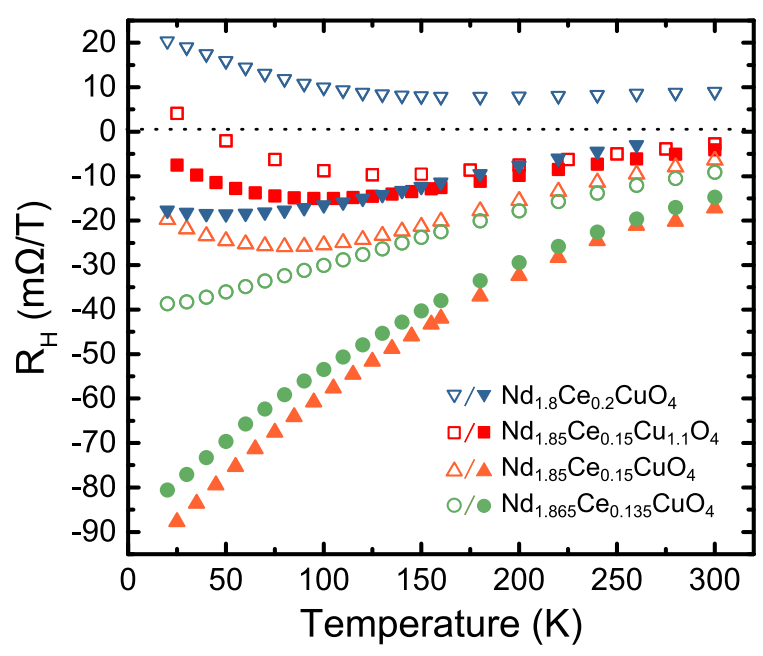

Figure A.1: Hall measurements on $70 \mathrm{~nm} \mathrm{Nd}_{2-\mathrm{x}} \mathrm{Ce}_{\mathrm{x}} \mathrm{CuO}_{4}$ films grown on $\mathrm{SrTiO}_{3}$. Annealing procedures standard (open symbols) and oxygen (closed symbols) are compared for doping levels $x=0.2(\nabla), x=0.15$ with extra copper $(\square)$ and without extra copper $(\triangle)$, and $x=0.135(\bigcirc)$.

The closed symbols show the effect of the additional oxygen annealing. Here, the same trend is observed as was also seen for the optimally doped films with the Hall coefficient shifting to more negative values upon oxygen annealing [105]. Comparing the open symbols to the closed symbols, we can see that the films grown from stoichiometric targets show a large change in the Hall coefficient between the two annealing procedures, whereas the film grown from the target with extra copper added (green squares) shows a much smaller change. 



\section{CHAPTER 3}

\section{LSCO/NCCO ramp-edge pn-junctions}

In-plane ramp-edge junctions between $\mathrm{Nd}_{1.85} \mathrm{Ce}_{0.15} \mathrm{CuO}_{4}$ (NCCO) and $\mathrm{La}_{1.85} \mathrm{Sr}_{0.15} \mathrm{CuO}_{4}$ (LSCO) have been fabricated where both layers are superconducting. At the interface, an insulating barrier is found in electronic transport. The barrier is found to be a tunneling barrier that shows a combination of elastic and inelastic tunneling, the former is indicated by the appearance of the LSCO phonon density of states in $d^{2} I / d V^{2}$ measurements and the latter is inferred from the temperature dependence. The energy scale of the barrier is smaller than would be expected from band alignment found by considering the cuprates as degenerate semiconductors and is closest to the scenario where hybridization of the $\mathrm{O} 2 p$ valence band states dictate band alignment. Additional experiments with overdoped interlayers of $\mathrm{Nd}_{2-\mathrm{x}} \mathrm{Ce}_{\mathrm{x}} \mathrm{CuO}_{4}$ and $\mathrm{La}_{2-\mathrm{x}} \mathrm{Sr}_{\mathrm{x}} \mathrm{CuO}_{4}$ show that the origin of the barrier is most likely a combination of electronic depletion mainly in the NCCO and a strain effect in the LSCO.

\subsection{Introduction}

Junctions of oppositely doped cuprates are predicted to have unusual properties. Theoretical work suggests that such junctions cross the Mott insulator ground state in a depletion zone at the interface [10-12]. We will refer to this as Mott depletion. The specific band alignment that is also discussed in chapter 1 can lead to Esaki diode behavior with negative resistance effects [10] and (super)conducting interface layers [12]. It can even lead to a system where the exact doping level of the two cuprates determines the full behavior of the junction, ranging from a normal Josephson contact or a tunneling barrier, to a situation where in a depletion zone the entire phase diagram is traversed and interface superconductivity can occur [12]. With the appearance of the Mott insulator ground state, a Josephson Mott diode is predicted [11], where the critical current can be modulated by the bias voltage.

In parallel, we are interested in using the combination of oppositely doped cuprates to find a system where the electrons and holes in the two materials can be brought into close proximity to explore excitonic effects in the rich phase diagram of the 
(a)
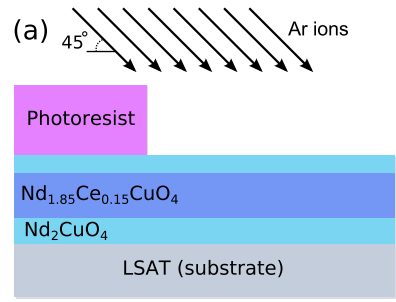

(b)

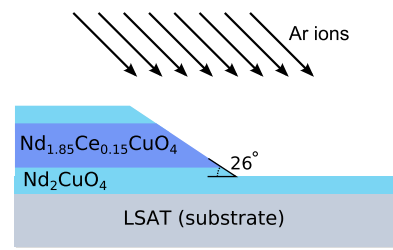

(c)

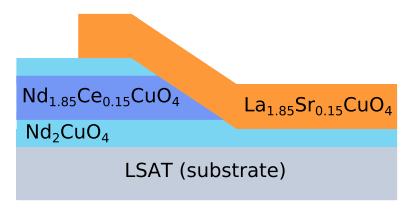

Figure 3.1: Schematic representation of the junction fabrication. (a) A ramp is defined in the bottom electrode stack by photo lithography and argon ion milling under an angle of $45^{\circ}$. (b) The etching stops in the $\mathrm{Nd}_{2} \mathrm{CuO}_{4}$ buffer layer and an in situ cleaning step is performed before deposition of the final LSCO layer. (c) Final structure of the LSCO/NCCO $p n$-junction, both superconductor layers are $150 \mathrm{~nm}$ thick.

cuprates (i.e. in an antiferromagnetic/superconducting background). This has led to various predictions ranging from strong localization effects of the excitons at low carrier densities [13] to Bose-Einstein condensation and phase separation of various ordered phases at finite densities $[14,15]$. The formation of excitons is also predicted to enhance the thermoelectric figure of merit [16].

Combinations of electron and hole-doped cuprates have previously been studied in the YBCO/NCCO system in various configurations [53-57]. Other than the appearance of a Josephson effect, none of the above described effects have been observed [55].

In this thesis, we focus on the combination of $\mathrm{La}_{2-\mathrm{x}} \mathrm{Sr}_{\mathrm{x}} \mathrm{CuO}_{4}$ and $\mathrm{Nd}_{2-\mathrm{x}} \mathrm{Ce}_{\mathrm{x}} \mathrm{CuO}_{4}$, two materials with a similar crystal structure and a more two-dimensional character in transport than e.g. YBCO, as also discussed in chapter 1. In this chapter we discuss the combination of electron-doped NCCO with hole-doped LSCO in rampedge junctions, where both layers are superconducting. The chapter will focus on sample characterization by X-ray diffraction and high-angle annular dark-field transmission electron microscopy (HAADF-STEM), and on electronic characterization of the junction formed at the interface between the two materials.

\subsection{Experimental details}

\subsubsection{Sample fabrication}

We have fabricated ramp-edge junctions between $\mathrm{Nd}_{1.85} \mathrm{Ce}_{0.15} \mathrm{CuO}_{4}(\mathrm{NCCO})$ and $\mathrm{La}_{1.85} \mathrm{Sr}_{0.15} \mathrm{CuO}_{4}$ (LSCO) $)^{\dagger}$ using pulsed laser deposition and ex- and in situ argon ion milling. The junctions are fabricated on edge aligned (001) oriented $\left[\mathrm{LaAlO}_{3}\right]_{0.3}\left[\mathrm{Sr}_{2} \mathrm{AlTaO}_{6}\right]_{0.7}$ (LSAT) substrates. The schematic sample fabrication

\footnotetext{
${ }^{\dagger}$ In the remainder of this chapter the acronyms NCCO and LSCO will refer to this exact doping level; other $\mathrm{Sr} / \mathrm{Ce}$ doping of materials used will be specified.
} 
is depicted in figure 3.1. As seen in figure 3.1(a), the bottom electrode consists of a $70 \mathrm{~nm} \mathrm{Nd} \mathrm{CuO}_{4}$ (NCO) buffer layer, a $150 \mathrm{~nm} \mathrm{NCCO} \mathrm{layer} \mathrm{and} \mathrm{a} 20 \mathrm{~nm} \mathrm{NCO}$ capping layer, all deposited at $820^{\circ} \mathrm{C}$ in 0.25 mbar oxygen. The NCCO layer is deposited from a non-stoichiometric target with extra copper added to suppress the formation of a copper poor parasitic phase, discussed in chapter 2. We use a buffer layer of NCO, as we have found that this reduces strain in the NCCO layer and more importantly, it improves the superconducting properties of the LSCO, which is used as the top electrode, see figure 3.1(c), over LSCO grown on an etched LSAT substrate; this is discussed in more detail in appendix C. The multilayer is subsequently cooled down to $740^{\circ} \mathrm{C}$ in deposition pressure and annealed in vacuum for $8 \mathrm{~min}$ followed by a cool down in vacuum at $10^{\circ} \mathrm{Cmin}^{-1}$. A ramp-edge is defined in this multilayer by using standard photo lithography and ex situ argon ion milling under a $45^{\circ}$ angle at $500 \mathrm{~V}$ acceleration voltage, followed by a cleaning step at $50 \mathrm{~V}$, see figure 3.1 (a). After removal of the photoresist, the ramp is cleaned by in situ argon ion milling, again at an angle of $45^{\circ}$, two pulses at $500 \mathrm{~V}$ and ten pulses at $50 \mathrm{~V}$, see figure $3.1(\mathrm{~b})$. The sample is then warmed up in vacuum to $700^{\circ} \mathrm{C}$, where the LSCO layer is deposited in 0.13 mbar oxygen, followed by a standard oxygen annealing in 1 bar oxygen with a 15 min dwell at $600{ }^{\circ} \mathrm{C}$ and a $30 \mathrm{~min}$ dwell at $450^{\circ} \mathrm{C}$. The LSCO layer is also patterned using standard photo lithography and argon ion milling, see figure 3.1(c). The device is contacted with sputter deposited gold/titanium contacts, see figure 3.2(a-b). The final $5 \times 5 \mathrm{~mm}$ chip consists of a $6 \times 3$ grid of junctions varying in width $(20,30,50,75,100$ and $150 \mu \mathrm{m})$. Two such junctions can be seen in figure 3.2(a). The widths are distributed randomly across the grid, to be able to discern the effects of junction width and sample uniformity. Each junction and its NCCO and LSCO leads can be independently measured using the voltage probes 1-4 in figure $3.2(\mathrm{~b})$.

A special sample was fabricated for dedicated high-angle annular dark-field scanning transmission electron microscopy (HAADF-STEM) measurements. Here, the bottom layer was structured into multiple $100 \mu \mathrm{m}$ wide lines and the subsequently deposited LSCO layer was not structured to facilitate HAADF-STEM sample preparation by focused ion beam (FIB).

\subsubsection{Structural characterization}

During sample fabrication the quality of the layers is monitored by X-ray diffraction (XRD). Figure 3.3(a) shows an XRD $\theta-2 \theta$ spectrum for the first NCONCCO-NCO multilayer stack compared to the spectrum after deposition of the final LSCO layer. The $(00 l)$ peaks for NCCO, LSCO and LSAT can be identified. Other peaks are also visible, mainly the parasitic $(\mathrm{Nd}, \mathrm{Ce})_{2} \mathrm{O}_{3}$ phase and three peaks attributed to a minor contribution of a misoriented LSCO phase, identified as (103), (110) and (200). Local diffraction near the junction area using a nanometer-sized beam has not revealed these reflections, see chapter 4 . This indicates that the misoriented phase originates from a polycrystalline phase, grown outside the junction area, likely near the edges of the sample. 

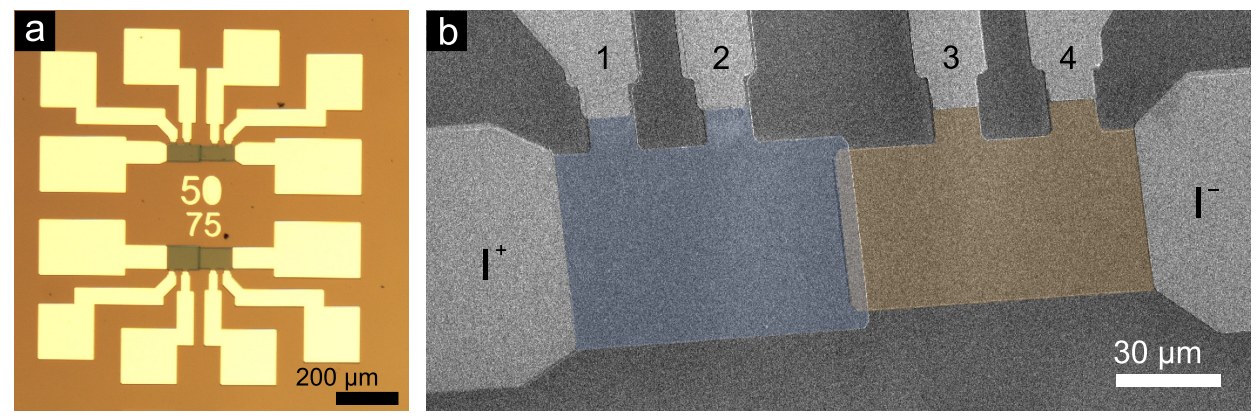

Figure 3.2: Overview of the $p n$-junction sample layout. (a) Optical micrograph of two 50 and $75 \mu \mathrm{m}$ wide $p n$-junctions, showing the electrode layout. (b) Scanning electron microscope close-up of a $50 \mu \mathrm{m}$ wide junction. The current leads are indicated and the voltage leads are numbered. False colors indicate the exposed NCCO (blue) and LSCO (orange) layers.

After each ion milling step, the etch depth is verified by atomic force microscopy (AFM) and the surface topography of the films is measured. The RMS roughness of the NCO-NCCO-NCO multilayer is generally larger than the LSCO layer, due to the more layer-by-layer-like growth mode of LSCO. We find an RMS roughness of $1.7 \mathrm{~nm}$ for the NCO-NCCO-NCO stack and $1.4 \mathrm{~nm}$ for the LSCO layer. Figure 3.3(b) shows an AFM close-up of the ramp in the bottom electrode and a profile across the ramp. The profile shows an angle of around $26^{\circ}$ for the main part of the ramp, where the LSCO layer will contact the NCCO in the multilayer stack. This angle deviates from the incoming beam angle and is determined by the ratio between the etch rate of the photoresist and the materials used via $\tan \alpha=\frac{1}{1+R} \tan \beta$, where $\alpha$ is the ramp angle, $\beta$ is the angle of the incoming beam and $R$ is the ratio between the etch rates of the photoresist and the NCCO layer [111]. For the combination of OLIN 907/17-906/12 photoresist and NCCO we find $R \approx 1$.

Figure 3.4(a-c) shows HAADF-STEM and energy dispersive X-ray spectroscopy (EDX) measurements on a LSCO/NCCO $p n$-junction. The general shape of the ramp-edge junction as shown in figure 3.1(c) and 3.3(b) can be recognized in figure 3.4(a). A sharp ramp is visible with an disordered area above the ramp due to redeposition during the ion milling process. The main ramp is also measured to have an angle of around $26^{\circ}$.

Figure 3.4(b) shows a close-up of the interface between the NCCO and LSCO layers. The LSCO layer shows a tilt of around three degrees with respect to the NCCO layer, this tilt will be discussed in more detail in chapter 4. A grain boundary is observed where the tilted phase meets the $c$-axis aligned phase away from the ramp, indicated by the black arrow in figure 3.4(a). The interface itself is relatively sharp with no crystalline phases other than LSCO and NCCO present. There is a possibility of the formation of the parasitic (Nd,Ce) ${ }_{2} \mathrm{O}_{3}$ phase during ion milling, due to excess heating and preferential sputtering of copper [112]. This is excluded by these TEM results and by a separate XRD experiment; for this, 
(a)

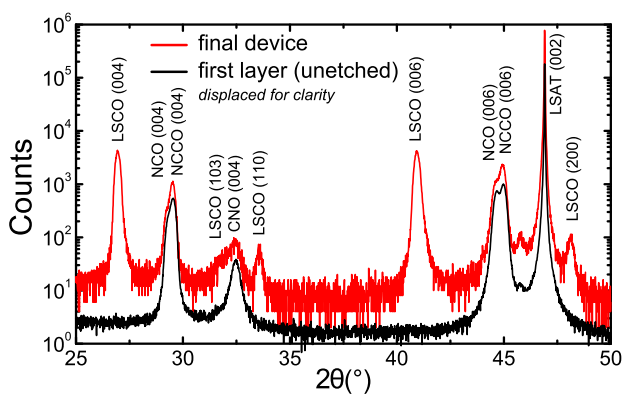

(b)

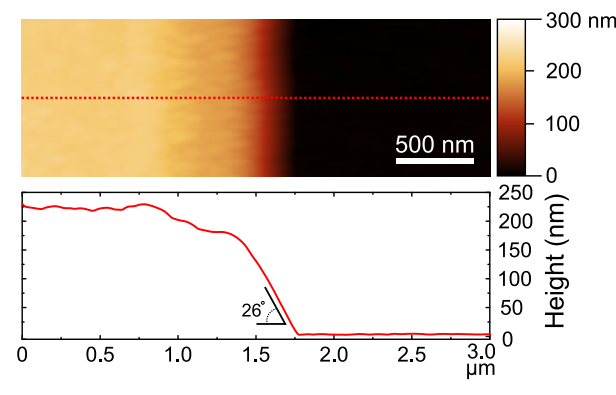

Figure 3.3: Sample characterization. (a) X-ray diffraction $\theta-2 \theta$ scan on the NCO-NCCO-NCO multilayer (black) and the final device (red), measured on the sample prepared for TEM. (b) AFM measurement of the ramp in the bottom layer. The red line shows a cross-section which shape can be recognized in figures 3.1(c) and 3.4(a).

the $(004)$ peak intensity of the $(\mathrm{Nd}, \mathrm{Ce})_{2} \mathrm{O}_{3}$ phase, normalized to LSAT $\left(\begin{array}{ll}0 & 0\end{array}\right)$, is compared before and after argon ion milling on a full NCCO film, and no change is observed.

At the interface, a changing HAADF background signal is observed. We attribute this to the intrinsic roughness of the ramp surface and strain relaxation in the LSCO layer. The roughness of the ramp can cause the exact position of the interface to vary across the thickness of the lamella. Roughness and the strain relaxation combined will lead to overlapping lattices, resulting in a shadowing effect at the interface. The signal cannot be caused by oxygen vacancies at the interface, since oxygen is generally not visible in HAADF-STEM, due to its relatively low mass [113]. The signal is also not caused by a misalignment of the ramp interface with the $\left[\begin{array}{lll}1 & 0 & 0\end{array}\right]$ zone axis as the $p n$-junction interface is edge aligned to the substrate to within one degree of accuracy. Assuming a typical thickness of the TEM lamella of $60-100 \mathrm{~nm}$, the observed size of $\sim 5 \mathrm{~nm}$ requires a misalignment of around three degrees. Therefore we conclude that the changing intensity is caused by the intrinsic roughness of the ramp surface and strain relaxation in the LSCO layer.

Figure 3.4(c) shows the EDX spectrum for the LSCO layer (red) and the NCCO layer (blue) of the $p n$-device near the $\mathrm{La}, \mathrm{Nd}$ and $\mathrm{Ce} L$-series $\left(L \alpha_{1,2}, L \beta_{1,2}\right.$ and $\left.L \gamma_{1}\right)$; the inset shows the EDX spectrum near the $\operatorname{Sr} K$-series $\left(K \alpha_{1,2}\right)$. The spectra are normalized to the low energy continuum intensity. The vertical lines indicate the positions of the spectral lines for each element. From the spectra we can see that the LSCO layer only contains $\mathrm{La}$ and $\mathrm{Sr}$ and no $\mathrm{Ce}$ or $\mathrm{Nd}$ has diffused into the top layer. In the $\mathrm{NCCO}$ spectrum $\mathrm{Nd}$ and Ce peaks can be identified and also a small contribution from La $L \alpha_{1,2}$, indicating some diffusion of La into the NCCO layer, most likely coming from the substrate. By quantitative analysis, the La content in the NCCO layer can be estimated to be of the order of $1 \%$ of the Nd concentration. The La is expected to act as an isovalent substitute of $\mathrm{Nd}$ 
in the NCCO and will mainly have an effect on the resistance through increased disorder and scattering. As can be seen from the inset in figure 3.4(c), no $\mathrm{Sr}$ is present in the NCCO layer. From the quantitative analysis we get a $\mathrm{Sr}$ content of $x=0.17 \pm 0.01$ for the $\mathrm{La}_{2-\mathrm{x}} \mathrm{Sr}_{\mathrm{x}} \mathrm{CuO}_{4}$ and a Ce content of $x=0.15 \pm 0.01$ for the $\mathrm{Nd}_{2-\mathrm{x}} \mathrm{Ce}_{\mathrm{x}} \mathrm{CuO}_{4}$.
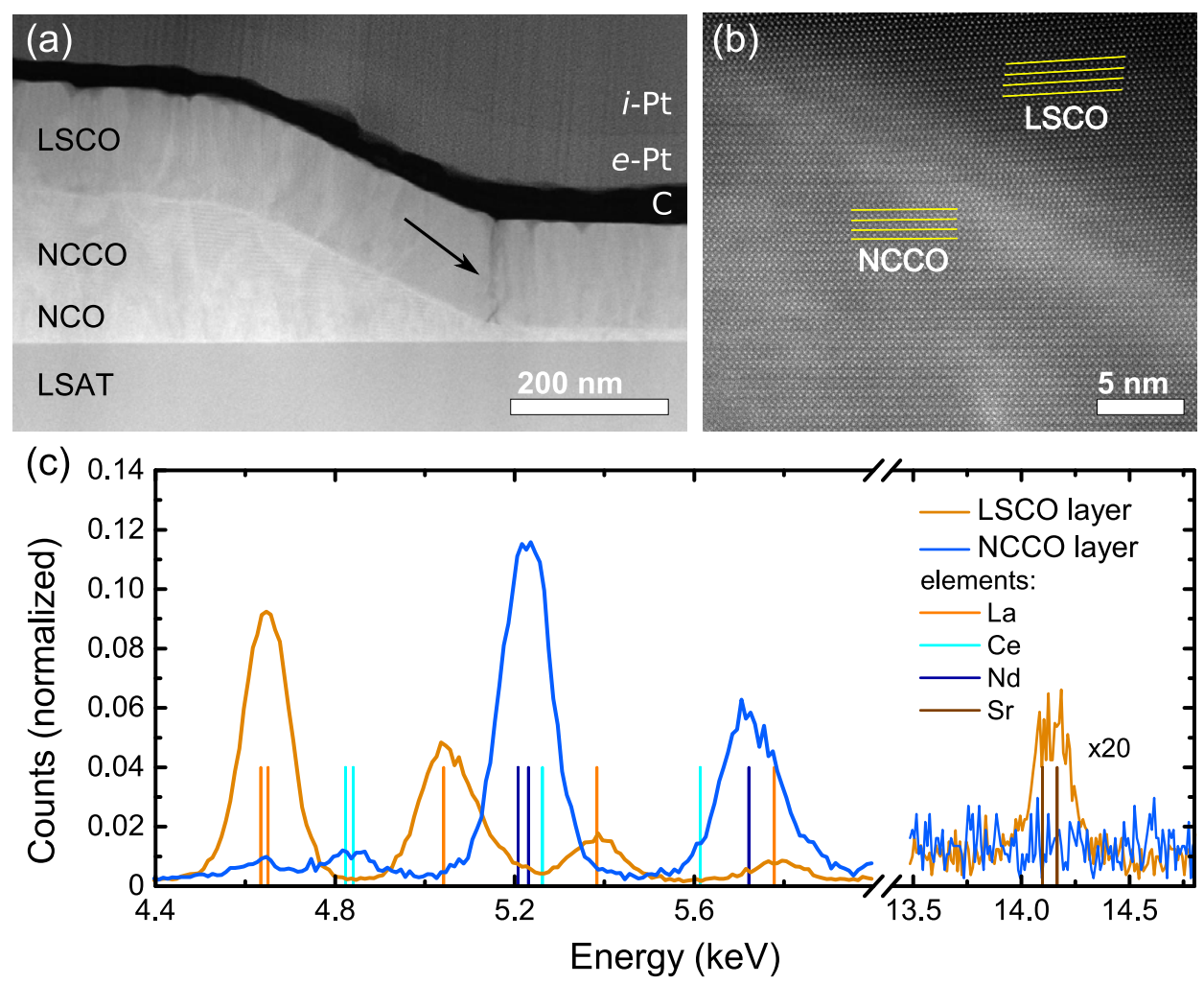

Figure 3.4: HAADF-STEM and EDX measurements on a NCCO-LSCO $p n$ junction. (a) Low magnification overview of the ramp-edge junction. The LSCO and NCCO layers are labeled, the black arrow indicates a grain boundary at the bottom of the ramp. The structure is capped with carbon $(\mathrm{C})$, and electron and ion-deposited $\mathrm{Pt}$ ( $e$-Pt and $i$-Pt respectively) used for the TEM sample fabrication by FIB. (b) Close-up of the ramp area. A clean interface is observed at the LSCO/NCCO boundary. The LSCO is tilted with respect to the NCCO lattice. (c) EDX spectra for the NCCO and the LSCO layers of (a) showing the elemental composition, normalized to the low energy continuum intensity. Spectral lines for $\mathrm{Nd}, \mathrm{La}, \mathrm{Ce}$ and $\mathrm{Sr}$ are indicated. 


\subsubsection{Measurement setup}

The devices were characterized electronically in a Quantum Design 9 T Physical Property Measurement System (PPMS), in a shielded bath cryostat (4.2 K and $1.3 \mathrm{~K}$, pumped) and in a flow cryostat (operated between $5 \mathrm{~K}$ and $300 \mathrm{~K}$ ). The latter two are equipped with dedicated home-built equipment for $I V$ measurements and RF irradiation. The PPMS is mainly used for basic sample characterization, i.e. resistance versus temperature measurements and preliminary $I V$ measurements. Detailed measurements were performed in the shielded cryostat, where multiple devices were characterized over different voltage bias regimes and under the influence of external sources like RF radiation and UV light ${ }^{\dagger}$.

\subsection{Results and discussion}

\subsubsection{IV measurements}

Figure 3.5(a) shows a resistance versus temperature measurement for the NCCO and the LSCO layer of the pn-devices, using contacts $1 \& 2$ and $3 \& 4$ of figure 3.2 (b), respectively. Both layers show a superconducting transition, where $T_{c}$ is close to the value we obtain for a single film for the LSCO layer $(19.3 \mathrm{~K})$ and about one degree lower for the NCCO layer $(15.5 \mathrm{~K})$, cf. the results in chapter 2. The latter difference is attributed to the thermal cycling during the deposition of the $\mathrm{La}_{1.85} \mathrm{Sr}_{0.15} \mathrm{CuO}_{4}$ layer. Measurements give a critical current density at $4.2 \mathrm{~K}$ of $5 \times 10^{8} \mathrm{~A} \mathrm{~m}^{-2}$ for the LSCO leads and $4 \times 10^{8} \mathrm{~A} \mathrm{~m}^{-2}$ for the NCCO leads.

We observe no Josephson supercurrent or onset of a supercurrent for the junctions down to $1.3 \mathrm{~K}$, see figure $3.5(\mathrm{~b}-\mathrm{d})$. Instead, we observe a strongly non-linear $I V$ characteristic, suggestive of a strong barrier at the interface. The $I V$ characteristic is measured by applying a sawtooth voltage bias to the sample and measuring the voltage across the junction and the voltage across a $100 \Omega$ or $1 \mathrm{k} \Omega$ resistance to get the applied current. The voltage bias regime is divided into bins that collect the average current for that voltage regime for all the periods of the sawtooth signal. The measurements in figures $3.5(\mathrm{~b}-\mathrm{d})$ use a bin size of $0.5 \mathrm{mV}$. The $d V / d I$ characteristics are obtained by a numerical derivative. For the $d^{2} I / d V^{2}$ characteristics, the data is smoothed using a five point moving average on the binned $I V$ data and once more using a three point moving average after the first numerical derivative. After the second numerical derivative, a background curve is subtracted. The background curve is determined by a 145 point Savitzky-Golay averaging of the $d^{2} I / d V^{2}$ curves for forward and reverse bias.

Figure 3.5(b) shows the $I V$ characteristics for four junctions with different junction width measured at $4.2 \mathrm{~K}$, with the current normalized to the width of the junction.

\footnotetext{
$\dagger$ Irradiation with microwave radiation between 6 and $18 \mathrm{GHz}$ and UV light from a high power blue LED $(470 \mathrm{~nm}, 2.64 \mathrm{eV}, 170 \mathrm{~mW})$ did not result in any measurable effects other than heating. Therefore, these experiments are not discussed further in this chapter.
} 
(a)

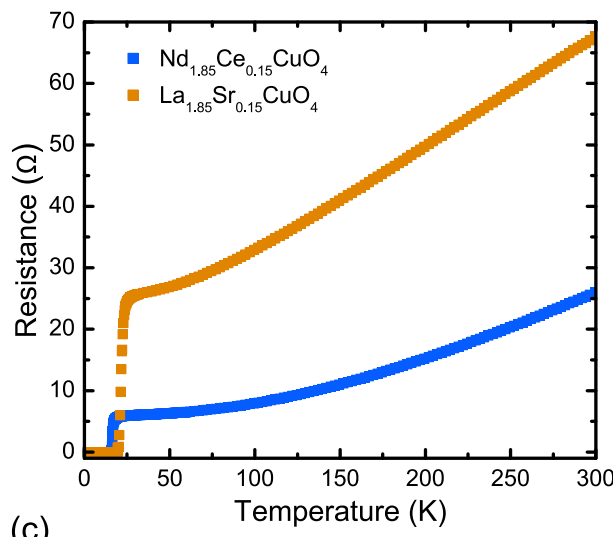

(c)

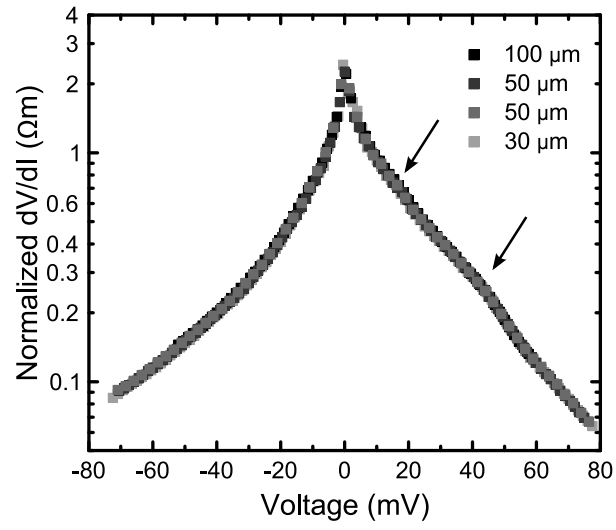

(b)

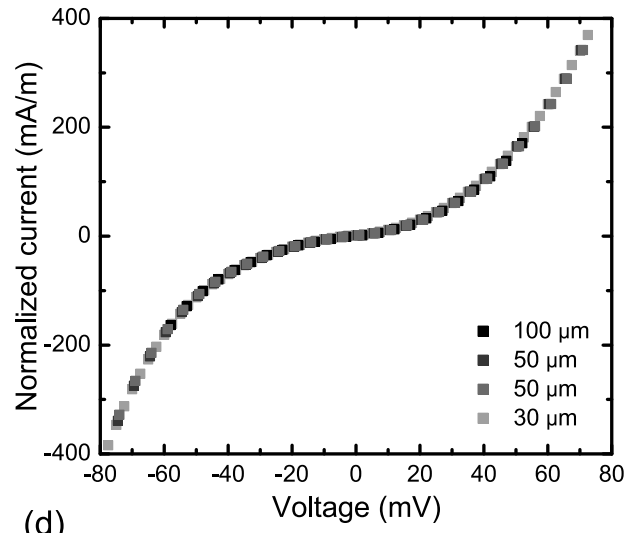

(d)

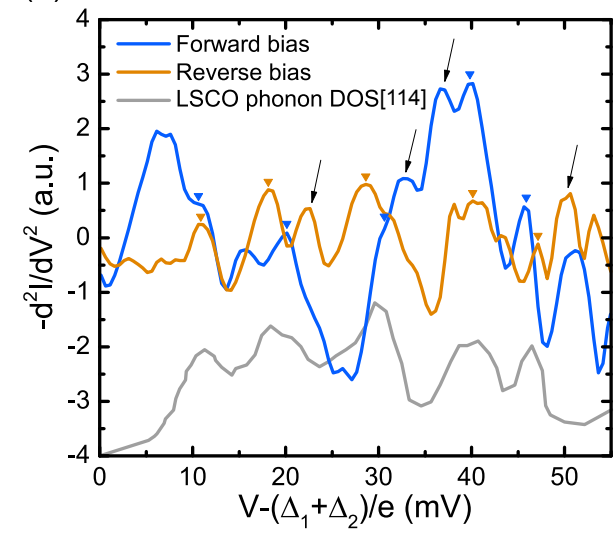

Figure 3.5: Overview of $I V$ measurements on LSCO/NCCO $p n$-junctions, (b), (c) and (d) are measured at $4.2 \mathrm{~K}$. (a) The NCCO layer and the LSCO layer both show a superconducting transition at low temperatures. (b) $I V$ characteristics with the current normalized to the width of the junction. The non-linear $I V$ curves are shown to scale with the width of the junction. (c) Numerical derivative of the curves in (b) show a pronounced asymmetry and the scaling from (b). Small features are seen in the forward bias branch, indicated by the black arrows. (d) $d^{2} I / d V^{2}$ curve, obtained by taking the numerical derivative of an $I V$ measurement. A smooth background is subtracted for easy comparison with the phonon density of states (DOS) of LSCO. Features are visible in the forward (blue) and reverse bias branch (orange). Most peaks can be attributed to inelastic tunneling with phonon modes of LSCO, a reference phonon DOS determined by inelastic neutron scattering is shown in gray [114]. The $d^{2} I / d V^{2}$ curves are displaced by $11.9 \mathrm{mV}$, the sum of the superconducting gaps of LSCO and NCCO $\left(\Delta_{1}+\Delta_{2}\right)$. Peaks that cannot be identified as either LSCO or NCCO phonon modes are indicated by black arrows. 
The curves follow the general shape expected for a $p n$-junction, albeit with only a small rectification. At large bias, we observe a lower resistance for forward bias. The $I V$ characteristic and the numerically derived resistance $(d V / d I)$, figure $3.5(\mathrm{c})$, scale with the width of the junction.

Figure 3.6(a) illustrates the asymmetry of the $d V / d I$ curve, forward (reverse) bias refers to a positive (negative) bias on the LSCO electrode. The $d V / d I$ curve shows the characteristic shape seen for a metal-insulator-metal (MIM) junction with an asymmetric tunnel barrier [80,115], figure $3.6(\mathrm{~b})$, where, after an initial overlap regime for small bias voltage $(<10 \mathrm{mV})$, the reverse bias branch first shows a lower resistance, followed by a crossover to a higher resistance than the forward bias branch. This indicates that the asymmetric barrier is lower on the NCCO side. A more detailed fitting using the metal-insulator-metal model will be discussed in $\S 3.3 .5$

\subsubsection{Tunneling spectrum with phonon modes}

Figure 3.5(c) shows a sharper resistance peak than generally observed for MIM junctions and additional spectroscopic peaks indicated by black arrows. We attribute these additional peaks to a modification of the density of states by electronphonon coupling [116]. Figure 3.5(d) shows a numerically derived $d^{2} I / d V^{2}$ curve for a $\mathrm{LSCO} / \mathrm{NCCO} p n$-junction to highlight the additional peaks and compares these to a reference of the LSCO phonon density of states (DOS) determined by inelastic neutron scattering shown in gray on an arbitrary scale [114]. In tunneling spectroscopy, the phonon modes are offset by the superconducting gap of both electrodes. Figure A.1 in appendix A shows a linear least squares fit with slope 1 to the first five peak positions of the phonon DOS and the corresponding features in the $-d^{2} I / d V^{2}$ spectrum for forward and reverse bias (indicated by the blue and orange triangles in figure 3.5(d)). From the $y$-axis cut-off, the sum of the superconducting gaps is determined to be $11.9(3) \mathrm{meV}$, see figure A.1 in appendix A. The expected size of the gap for NCCO is $2.7 \mathrm{meV}$, using $2 \Delta(0) / k_{B} T_{c}=4.1$ [117]. This results in a gap of $9.2 \mathrm{meV}$ for LSCO, which is comparable to what is seen in literature [118-120]. For comparison to the phonon density of states, the $-d^{2} I / d V^{2}$ curves are therefore shifted by $11.9 \mathrm{mV}$ in figure $3.5(\mathrm{~d})$.

As is also observed in figure 3.5(c), the signal for reverse bias is weaker than the forward bias signal, when electrons are tunneling into the LSCO layer. This is also observed in Al-LSCO tunneling experiments, but not as strongly [118]. Inelastic neutron scattering is sensitive to light atoms like oxygen, whereas tunneling is sensitive to the electron-phonon coupling; this explains the difference in relative strength of the modes when comparing the curves in figure $3.5(\mathrm{~d})$ to the reference data [114]. No features related to phonons in NCCO from either inelastic neutron scattering data [121] or Raman spectroscopy [122] can be identified in the $-d^{2} I / d V^{2}$ curves, still assuming a shift of $11.9 \mathrm{mV}$. The sharpest feature in the inelastic neutron scattering data for $\mathrm{NCCO}$ appears at $13 \mathrm{meV}$, which falls between two peaks in our $-d^{2} I / d V^{2}$ measurements. 
No peaks can be attributed to phonon modes in NCCO [121], except when there is an overlap with the LSCO modes. There are four peaks visible that are not associated with the phonon spectrum as measured by Renker et al. [114], indicated by the black arrows in figure 3.5(d). Only the peak at $50.5 \mathrm{mV}$ appears for both forward and reverse bias. These peaks are all also observed in point contact spectroscopy on polycrystalline LSCO using $\mathrm{Al}$ electrodes. Here, they are attributed to specific enhancement of the electron-phonon coupling, gap anisotropy or modification of the phonon energies at the interface [118].

It has already been shown that at low energy the relevant bosons in LSCO are phonons [120]. Using our experiment, we confirm this result and also show that there is no strong electron-phonon coupling in $\mathrm{NCCO}$ for our devices. The latter is in contrast to the observations of e.g. Huang et al. [123]. Furthermore, Shim et al. [120] show that the inelastic tunneling spectrum of LSCO is dominated by copperoxygen and oxygen related phonons with no contribution from $\mathrm{La}(\mathrm{Sr})$ vibrations. Our data does show the modes associated with the $\mathrm{La}(\mathrm{Sr})$ vibrations, centered around $28 \mathrm{mV}$. This might be explained by the different measurement geometry, a ramp-edge junction geometry versus a bicrystal grain boundary junction. The bicrystal grain boundary is a purely in-plane contact, whereas in a ramp-edge junction there is also a possible contribution in the $c$-axis direction along the ramp.

One could argue that the observed spectrum is caused by the LSCO-LSCO grain boundary we observe at the bottom of the ramp, see figure 3.4(a). This is not very likely for several reasons: first of all, the grain boundary resistance is expected to be very low, because the small tilt of the LSCO lattice as compared to e.g. the $24^{\circ}$ tilt used by Shim et al. [120]; secondly, in the case of an LSCO-LSCO grain boundary, the phonon spectrum is expected to have equal signal strength for forward and reverse bias, which is clearly not the case in our experiment; and finally, the phonon spectrum is expected to shift by twice the gap of LSCO, $\sim 18 \mathrm{meV}$, which does not result in a good match between the observed spectrum and the phonon DOS reference data.

\subsubsection{Origin of the barrier}

The barrier at the LSCO/NCCO interface can have multiple origins: (1) an electronic depletion zone between the $n$-type NCCO and the $p$-type LSCO [10,12]; (2) strain in the LSCO layer; (3) damage on the ramp-edge; (4) oxygen diffusion across the interface; and (5) the tilt grain boundary between either the NCCO and the LSCO or in the LSCO layer itself, at the bottom of the ramp in figure 3.4(a). The last three points can be addressed individually, the first two will be discussed in the next sections.

The tilt of the LSCO lattice on the ramp is not expected to result in a large resistance at either the $\mathrm{LSCO} / \mathrm{NCCO}$ interface or where the tilted LSCO meets the $c$-axis aligned LSCO, see figure 3.4(a). The tilt is only $3^{\circ}$, Josephson junctions have been fabricated using much larger tilts. YBCO can form a Josephson contact 
(a)

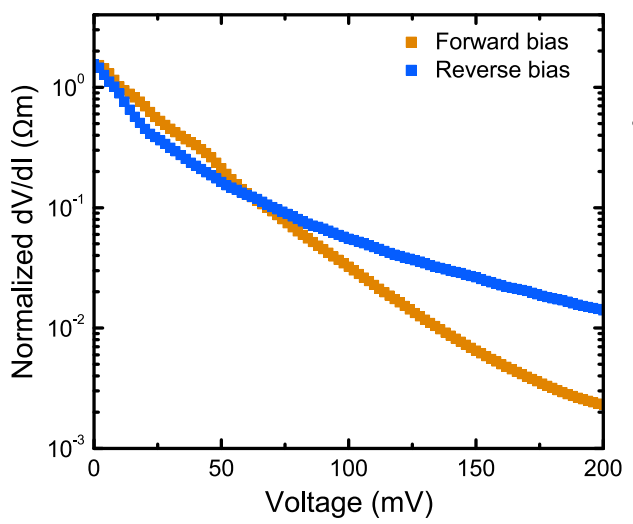

(b)

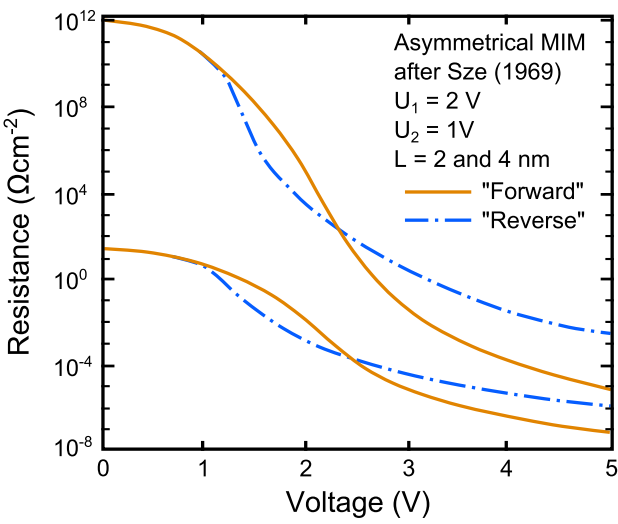

Figure 3.6: Metal-insulator-metal (MIM) junction behavior for the LSCO/NCCO $p n$-junctions. (a) Normalized $d V / d I$ versus absolute voltage bias for forward and reverse bias showing a clear asymmetry and the characteristic behavior for a MIM junction. (b) Asymmetric MIM junction resistance versus voltage adapted from Sze, Physics of Semiconductor Devices [80], showing the same crossover behavior as is seen for the $p n$-junctions in (a).

even when the $\mathrm{CuO}_{2}$ planes tilt $45^{\circ}$ at a grain boundary [124]. Using LSCO, a grain boundary with a tilt of $24^{\circ}$ results in a tunneling contact with a typical resistance of $100 \Omega$ or less for dimensions comparable to the device dimensions used in this work [120]. A $3^{\circ}$ tilt is expected to show a much smaller resistance and more likely, a Josephson contact or even a superconducting short.

Oxygen diffusion is certainly something to take into account at the interface between NCCO and LSCO. From the basic crystal structure one would expect that the LSCO T-phase tends to attract oxygen and that oxygen is easily removed from the NCCO $T^{\prime}$-phase. If there is oxygen diffusion at the interface it is most likely going from $\mathrm{NCCO}$ to LSCO, driving both materials toward over-doping (i.e. away from the undoped parent compound). If oxygen diffusion takes place in the other direction, the situation is different. Now both the LSCO and the NCCO are driven towards under-doping. Even more critical, the oxygen vacancies in the $\mathrm{CuO}_{2}$ planes of $\mathrm{NCCO}$, created by vacuum annealing, might be filled again, completely suppressing superconductivity. This second scenario cannot be fully excluded, but it is not likely from a crystal stability perspective. LSCO does not as easily lose oxygen as for example $\mathrm{YBCO}$, where oxygen can be easily removed from the $\mathrm{Cu}-\mathrm{O}$ chains, as argued by Alff et al. for their $\mathrm{YBCO} / \mathrm{NCCO}$ junctions [57]. Some information might be obtained from an EDX map near the oxygen K-edge to determine the oxygen content of both NCCO and LSCO near the ramp interface.

Overall, no variation of the oxygen signal is observed across the device using EDX. Another way to investigate this would be to look at the LAADF (lowangle annular dark-field) STEM signal, which is also sensitive to scattering from 
oxygen vacancies [113]. Although this signal may be obscured by scattering off the amorphous structure found at the ramp interface and off carbon deposits on the TEM sample.

Damage on the ramp-edge is investigated by looking at homojunctions of only LSCO or only NCCO. Junction fabrication and initial characterization is described in appendix B. In both cases a Josephson weak-link is observed as evidenced by the presence of a supercurrent and the appearance of Shapiro steps under microwave irradiation, see figure 3.7. It is important to note that the homojunctions do not form a superconducting short; the appearance of a Josephson supercurrent suggests the presence of a barrier or a weak link at the interface. Especially in the NCCO case, figure 3.7(b), the critical current is low and only present in a sample that is heated up in deposition pressure for the deposition of the final superconducting layer. A sample where the first set of layers was heated up in vacuum only shows a resistance dip around zero bias with peaks associated with the gap and Andreev reflection. Both cases show an $I_{c} R_{N}$ product, where $I_{c}$ is the critical current and $R_{N}$ is the normal-state resistance, at least an order of magnitude below the gap, suggesting that there is a barrier at the interface. However, the normal state resistance is on the order of $1 \Omega$ or less for both cases, at least four orders of magnitude smaller than the barrier observed for the LSCO/NCCO junctions. For a $75 \mu \mathrm{m}$ wide LSCO/NCCO $p n$-junction we find a differential resistance of $50 \mathrm{k} \Omega$ for zero bias. With a film thickness of $150 \mathrm{~nm}$, this gives a barrier resistivity of $5.6 \times 10^{-3} \Omega \mathrm{cm}^{2}$, to be compared to a barrier resistivity of less than $10^{-7} \Omega \mathrm{cm}^{2}$ for LSCO/LSCO or NCCO/NCCO junctions of the same geometry or a resistivity of $10^{-7}-10^{-8} \Omega \mathrm{cm}^{2}$ for YBCO grain boundaries $[125,126]$. The only barrier expected at the interface of a homojunctions is through damage or (oxygen) off-stoichiometry at the ramp interface. This effect is seen in the LSCO/NCCO $p n$-junctions as the amorphous background near the ramp interface in the HAADF-STEM measurements, see figure 3.4(b). From the measurements on the homojunctions we can conclude that damage at the ramp interface does not lead to resistances as large as what we observe for the LSCO/NCCO $p n$ junctions.

\subsubsection{Disentangling strain and electronic effects}

Strain effects cannot be completely ruled out, but in the entire junction LSCO is grown on either $\mathrm{NCCO}$ or $\mathrm{NCO}$, so the same strain that exists at the interface is also present across the whole sample. It is possible that a thin layer of the LSCO is insulating, but the electrodes are fully superconducting. We have found that growing LSCO on NCCO instead of e.g. on LSAT, does not influence $T_{c}$ in a major way. Strain in the LSCO layer can have a potential role at the ramp-edge interface. From HAADF-STEM we get a typical strain relaxation length of $3-5$ $\mathrm{nm}$. The energy scale for a strain induced barrier would be related to the insulating gap of the parent compound or the position of the Fermi level with respect to the conductance or valence band. From figure 1.5 in chapter 1 this energy scale would then lie between $0.4 \mathrm{eV}$ and $1.5 \mathrm{eV}$. 
(a)

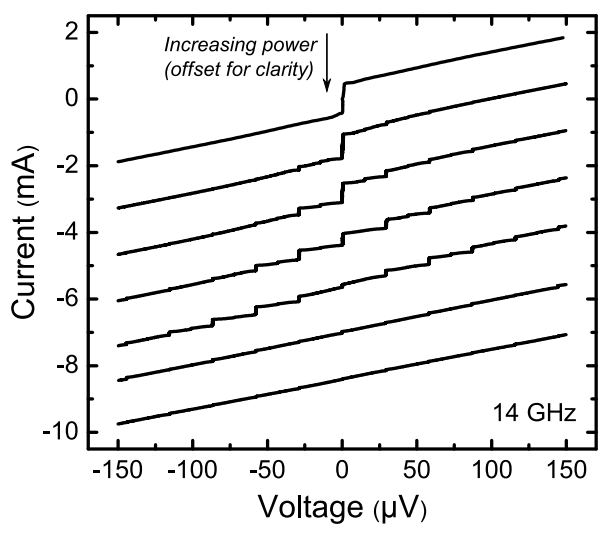

(b)

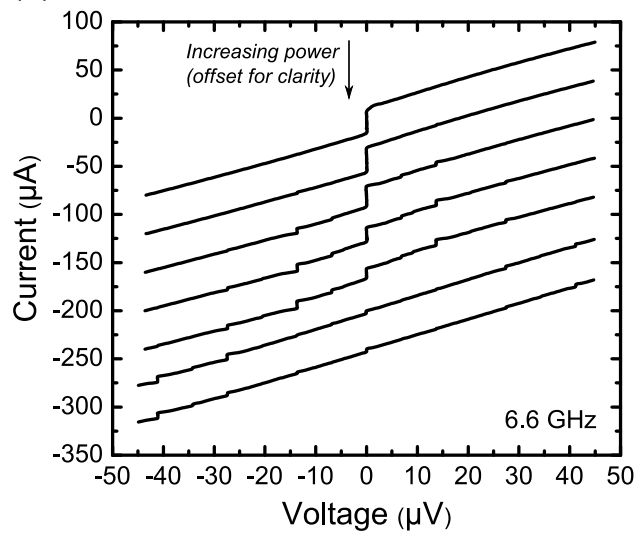

Figure 3.7: Ramp-edge homojunctions showing Josephson supercurrents, evidenced by the appearance of Shapiro steps upon RF irradiation. Results shown for $\mathrm{La}_{1.85} \mathrm{Sr}_{0.15} \mathrm{CuO}_{4}-\mathrm{La}_{1.85} \mathrm{Sr}_{0.15} \mathrm{CuO}_{4}$ (a) and $\mathrm{Nd}_{1.85} \mathrm{Ce}_{0.15} \mathrm{CuO}_{4}$ $\mathrm{Nd}_{1.85} \mathrm{Ce}_{0.15} \mathrm{CuO}_{4}$ (b); $\mathrm{RF}$ power increases from top to bottom, curves have been offset for clarity.

In chapter 1 we have discussed two scenarios for band alignment between NCCO and LSCO. For an Anderson/Schottky-Mott type band alignment dictated by the work functions of NCCO and LSCO, we find an expected built-in potential of $1.53-1.63 \mathrm{eV}$. For a band alignment dictated by a hybridization of the valence band oxygen $2 p$ states, we find a built-in potential of $0.5-0.7 \mathrm{eV}$. We have estimated the size of the depletion zone using Poisson's equation for degenerate semiconductors and find $1-4 \mathrm{~nm}$ and $0.5-2.5 \mathrm{~nm}$, respectively.

It might be quite difficult to disentangle the effect of electronic depletion due to the $p n$-nature of the contact and the depletion effect of strain in the LSCO layer. In order to address the role of both strain, the next sections will discuss a metalinsulator-metal model for the $p n$-junctions, to get an estimate of the energy and length scales associated with the barrier, we will look at the effect of interlayers of overdoped $\mathrm{Nd}_{2-\mathrm{x}} \mathrm{Ce}_{\mathrm{x}} \mathrm{CuO}_{4}$ and $\mathrm{La}_{2-\mathrm{x}} \mathrm{Sr}_{\mathrm{x}} \mathrm{CuO}_{4}$ and we will discuss the temperature dependence of the junctions.

\subsubsection{Metal-insulator-metal modeling}

As seen in figure 3.6, the general shape of the $I V$-characteristics matches that of a MIM junction. Therefore, we fit the curves by a tunneling model using the Wentzel-Kramers-Brillouin (WKB) approximation for tunneling across an asymmetric barrier of length $L$ and a potential that varies linearly from $U_{1}$ at $x=0$ to $U_{2}$ at $x=L$, see figure $3.8($ a) $[80,115]$. The WKB approximation assumes a slowly varying potential compared to the wavelength of the incoming electrons. In doing so we can write down the wavefunction for a one dimensional situation for 
each point in space as

$$
\Psi(x)=A(x) \mathrm{e}^{i \phi(x)},
$$

where $A(x)$ is the amplitude of the wavefunction and $\phi(x)$ the phase. Plugging this into the Schrödinger equation (using $k(x)=\sqrt{2 m[E-U(x)]} / \hbar$, where $E$ is the energy, $m$ is the effective mass and $U(x)$ is an arbitrary, but slowly varying potential), the phase factor is found to be

$$
\phi(x)= \pm \int k(x) \mathrm{d} x
$$

In the barrier region $E<U(x)$ and $\phi(x)$ becomes imaginary, resulting in a decaying mode. The transmission is found from the ratio of the transmitted probability and the incoming probability and amounts to

$$
T(E)=\exp \left[-2 \int_{0}^{L}|k(x)| \mathrm{d} x\right],
$$

where $U(x)$ is determined by $U_{1}, U_{2}$ and $L$, see figure 3.8(a). For an applied bias $V$, the current (apart from a scaling factor) is found by integrating $T(E)$ :

$$
I \propto \int_{0}^{\infty} T(E)[F(E)-F(E+e V)] \mathrm{d} E,
$$

where $F(E)$ is the Fermi distribution. At the measurement temperatures $k_{B} T$ broadening is negligible, so the Fermi distributions of equation (3.4) are approximated by step functions. The final shape of the $I V$ characteristic depends not only on barrier parameters $U_{1}, U_{2}$ and $L$ but also on the effective mass. The effective mass for NCCO and LSCO varies from $1-5 m_{e}[127,128]$. In the following we will only consider $m=m_{e}$, to keep the model simple and to avoid additional fitting parameters. Therefore, the model yields order of magnitude values for the barrier height and width. Figure 3.8(b) shows a fit to the $I V$ characteristic from figure 3.5 up to a higher bias, using a numerical simulation of the model. Here, the barrier parameters $U_{1}, U_{2}$ and $L$ and a scaling factor for the current are optimized using a least-squares minimization routine. The energy scale found using this model is mainly dominated by the barrier variables $U_{1}$ and $U_{2}$, where $U_{1}=35 \mathrm{meV}$ and $U_{2}=83 \mathrm{meV}$. The barrier width is found to be $5.4 \mathrm{~nm}$. Figure $3.8(\mathrm{c})$ gives an indication for the possible spread in fitting parameters by plotting a simulation for values $5 \%$ larger (red) and smaller (blue) than the optimized values, leaving the scaling factor as a free parameter.

The model fit only captures the general characteristics of the $p n$-junction, see figure 3.8(b). Especially in the sub-barrier regime, the model underestimates the magnitude of the current. This cannot be caused by thermal rounding of the barrier or of the density of states near the Fermi level, as $k_{B} T$ is sub-meV at the measurement temperatures. In the model it is assumed that the density of states 
(a)

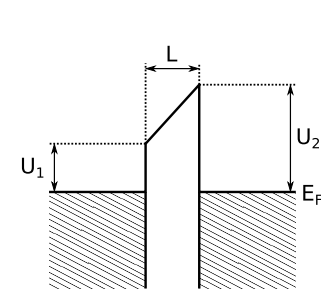

(b)

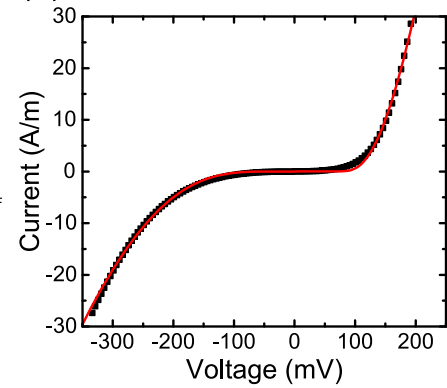

(c)

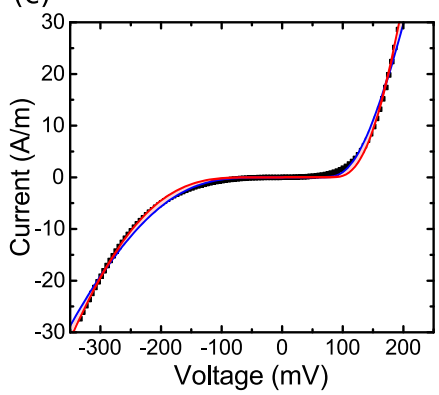

Figure 3.8: WKB tunneling model. (a) Schematic of the asymmetric barrier with parameters $U_{1}, U_{2}$ and $L$ indicated. (b) WKB tunneling fit to the $I V$ characteristic of a LSCO/NCCO $p n$-junction $\left(U_{1}=35 \mathrm{meV}, U_{2}=83 \mathrm{meV}\right.$ and $L=5.4 \mathrm{~nm}$ ). (c) Error range of the model using model parameters $5 \%$ lower (blue) and $5 \%$ higher (red) than the values used in (b). The scaling factor is left as a fitting parameter.

is constant across the whole bias regime, which might not be true. The deviation might also be caused by the underlying two-carrier nature of the cuprates near optimal doping. The pn-junction would then essentially consist of two parallel conduction channels; each of these can have a different energy barrier for transport. Another possibility is a deviation from the Fermi distribution for states around the Fermi level; inelastic scattering at the interface can introduce a broadening of several meV of the states around the Fermi level $[129,130]$. In section 3.3 .7 we show that the temperature dependence of the junctions is dominated by inelastic tunneling transport. Since we argue that the barrier originates from band bending or strain effects, it is also reasonable to assume that the barrier is not as sharp as we represent in the model. A smoother barrier shape would also result in rounding of the $I V$ characteristic. On top of that, in real MIM contacts, the effective barrier is a rounded version of the idealized square barrier due to image-force effects at the metal interfaces [115]. Overall we can conclude that the simple model gives an order of magnitude estimate for the barrier parameters and that the real values, before rounding and image-force effects, will be somewhat larger.

The energy scale found in the MIM modeling is smaller than either of the energy scales expected from the band alignment models. The valence band hybridization scenario is the closest in energy. It is also smaller than the energy scale expected from a strain induced barrier. The width of the barrier has the same order of magnitude in both the strain and the electronic depletion scenarios, and it is comparable to what we find from the model. The fit clearly does not capture the full physics of the device and analysis of the $p n$-junction in terms of electronic effects relies heavily on the assumption that the cuprates can be approximated as degenerate semiconductors. The validity of the calculations in section $\S 1.3 .6$ of chapter 1 is debatable, as the continuum limit approach of Poisson's equation breaks down when the typical length scales approach inter-atomic distances. It also completely neglects the strong interactions that govern the (doped) Mott 
(a)

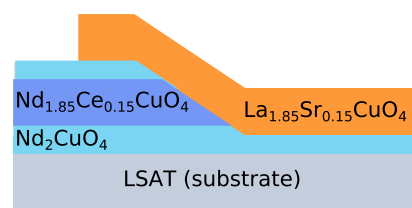

pn (b)

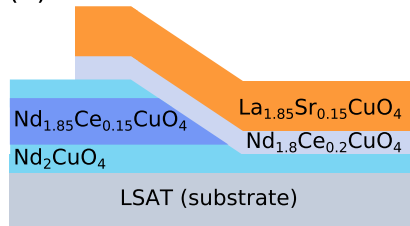

$p n^{\prime} n$ (c)

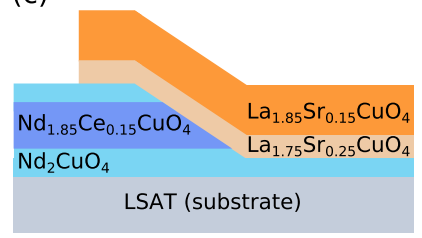

$p p^{\prime} n$

Figure 3.9: Schematic cross-section of the $p n$ (a), the $p n^{\prime} n$ (b) and the $p p^{\prime} n$ (c) configuration. The interlayer thickness is $20 \mathrm{~nm}$.

insulator state. The calculated parameters should therefore only be taken as order of magnitude estimates. Based on this, we cannot definitively conclude which of the two effects, or both, plays a role in the physics of the LSCO-NCCO $p n$ junctions.

Therefore, from the energy and length scale we cannot distinguish between an electronic or strain origin for formation of the barrier. However, the barrier shape also gives some information. We find that the barrier is lower on the side of the NCCO. For a strain induced effect it is expected that the barrier is fully developed at the NCCO side and relaxes into the LSCO, resulting in a higher barrier parameter on the NCCO side than on the LSCO side. The barrier shape is harder to estimate for the electronic origin. From the general phase diagram of the cuprates we know that the antiferromagnetic Mott insulator ground state extends to higher doping on the electron doped side, as was discussed in chapter $1^{\dagger}$. It is therefore expected that the band gap is more developed on the NCCO side of the junction and that the LSCO layer behaves more like a metal. In this scenario most of the band bending due to the Fermi level mismatch would occur in the NCCO layer. A barrier due to a depletion effect would then be sharp at the LSCO/NCCO interface and decay into the NCCO. This comparable to the barrier shape we find using the MIM model.

\subsection{6 pn-junctions with different interlayers}

Further investigation into the nature of the $p n$ contact was done by including $20 \mathrm{~nm}$ overdoped interlayers of $\mathrm{Nd}_{1.8} \mathrm{Ce}_{0.2} \mathrm{CuO}_{4}$ and $\mathrm{La}_{1.75} \mathrm{Sr}_{0.25} \mathrm{CuO}_{4}$, see figure 3.10 (a). These were initially chosen to provide a layer of mixed carrier type as an intermediary between the oppositely doped cuprates with the idea that this might induce a Josephson contact. This turned out not to be the case and moreover, both $\mathrm{Nd}_{1.8} \mathrm{Ce}_{0.2} \mathrm{CuO}_{4}$ and $\mathrm{La}_{1.75} \mathrm{Sr}_{0.25} \mathrm{CuO}_{4}$ show a positive Hall coefficient down to $2 \mathrm{~K}$ and up to $9 \mathrm{~T}$ with no indication of mixed carriers. Both interlayer materials have a doping level that puts them at the overdoped edge of the superconducting

\footnotetext{
$\dagger$ Electrons are doped into the $\mathrm{Cu} 3 d$ upper Hubbard band and only dilute the antiferromagnetic ordering, holes initially reside in the $\mathrm{O} 2 p$ band (before forming Zhang-Rice singlets), thereby frustrating the ordering of the electron spins in the half filled $\mathrm{Cu} 3 \mathrm{~d}$ lower Hubbard band [29].
} 
(a)

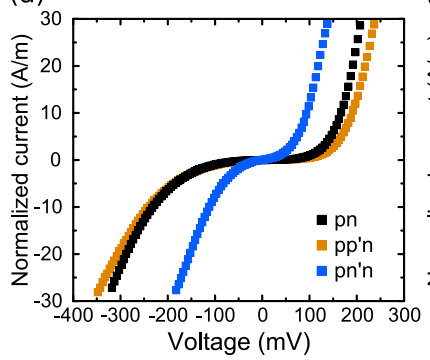

(b)

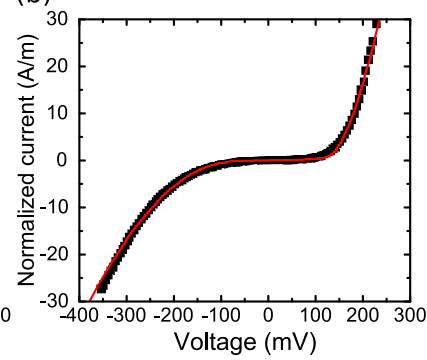

(c)

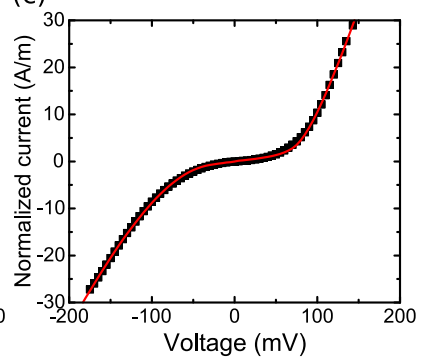

Figure 3.10: $I V$ characteristics for different interlayers. (a) $p n$ configuration compared to the $p p^{\prime} n$ configuration with a $\mathrm{La}_{1.75} \mathrm{Sr}_{0.25} \mathrm{CuO}_{4}$ interlayer and $p n^{\prime} n$ with a $\mathrm{Nd}_{1.8} \mathrm{Ce}_{0.2} \mathrm{CuO}_{4}$ interlayer. The current is normalized to the junction width. (b) WKB tunneling fit (red line) for the $p p^{\prime} n I V$ characteristic $\left(U_{1}=\right.$ $48 \mathrm{meV}, U_{2}=124 \mathrm{meV}$ and $L=2.9 \mathrm{~nm}$ ). (c) WKB tunneling fit (red line) for the $p n^{\prime} n I V$ characteristic $\left(U_{1}=35 \mathrm{meV}, U_{2}=71 \mathrm{meV}\right.$ and $\left.L=2.2 \mathrm{~nm}\right)$.

dome; both show an inhomogeneous superconducting transition that never fully reaches zero resistance. Here, we use these interlayers to study their effect on the transport across the $p n$-interface.

In the following we will compare three different configurations: no interlayer $(p n)$, a $\mathrm{Nd}_{1.8} \mathrm{Ce}_{0.2} \mathrm{CuO}_{4}$ interlayer $\left(p n^{\prime} n\right)$ and a $\mathrm{La}_{1.75} \mathrm{Sr}_{0.25} \mathrm{CuO}_{4}$ interlayer $\left(p p^{\prime} n\right)$, see figure 3.9. For both interlayers the general shape and asymmetry of the $I V$-curves remains. For the $\mathrm{La}_{1.75} \mathrm{Sr}_{0.25} \mathrm{CuO}_{4}$ interlayer (orange curve), we see an increase in the apparent barrier height, but the shape of the $I V$-curve can be scaled to the original $p n$-junction, in particular for the forward bias branch. In the WKB tunneling simulations, small changes in the width of the junction have a symmetric effect on the scalability of the two branches. A difference in scalability can only be explained by a change in the barrier height and in particular by a change in the ratio $U_{1} / U_{2}$. The addition of an overdoped $\mathrm{Nd}_{1.8} \mathrm{Ce}_{0.2} \mathrm{CuO}_{4}$ interlayer (blue curve) results in a decrease of the resistance at zero bias of more than an order of magnitude. The resulting $I V$-curve still shows an asymmetry, but it does not scale to the original $p n$-junction.

Figures 3.10(b-c) show the WKB tunneling fit for the $p p^{\prime} n$ (b) and the $p n^{\prime} n$ (c) $I V$ characteristics. From the fitting we see that the $p p^{\prime} n$ configuration has a smaller barrier width and a larger barrier height, with a larger increase for the $U_{2}$ parameter associated with the LSCO electrode. The $p n^{\prime} n$ configuration shows both a smaller barrier height and barrier width. 


\subsubsection{Temperature dependence}

Figure 3.11(a-b) shows the temperature dependence of the conductivity at zero bias for the $p n, p p^{\prime} n$ and $p n^{\prime} n$ configuration ${ }^{\dagger}$. At low temperatures, the conductivity scales with $T^{4 / 3}$, characteristic for inelastic tunneling dominated by two impurity hopping as described by Glazman and Matveev [131]. For higher temperatures the conductivity deviates from the $T^{4 / 3}$ dependence and is seen to increase with the square of the temperature up to around $100 \mathrm{~K}$ for all configurations. Above this temperature, conductance through the buffer layer also becomes significant. Note that the conductance of the $p n$ configuration is lower than that of the $p p^{\prime} n$ or the $p n^{\prime} n$ configuration. From the WKB tunneling model we have a lower barrier for the $p n$ configuration compared to the $p p^{\prime} n$ configuration, but the barrier width is larger. The conductance of a tunneling contact scales with the square of the temperature. It factors in through the temperature dependence of the Fermi distribution of both electrodes, which has a $T^{2}$ leading order [132], where the slope is proportional to $d^{2} / \bar{U}$, with $\bar{U}$ the average barrier height. We find the same order of magnitude when we normalize the curves to the extrapolated resistance at zero bias as would be expected from the fit parameters of figures 3.8 and 3.10, with the largest value for the $p n$ configuration and the lowest for the $p n^{\prime} n$ configuration. The temperature dependence cannot be caused by the respective $T$ and $T^{2}$ temperature dependence of the LSCO and NCCO leads above $T_{c}$, since the lead conductance increases with decreasing temperature. The resistance change in the temperature interval of figure 3.11 is at least $1 \mathrm{k} \Omega$ for all configurations, much larger than the resistance change of the leads seen in figure 3.5(a).

At the lowest temperatures the conductivity for the $p n^{\prime} n$ and the $p p^{\prime} n$ configuration deviate from the $T^{4 / 3}$ dependence towards a dependence linear in temperature. The conductance of the $p n$-junction shows a $T^{4 / 3}$ behavior down to $2 \mathrm{~K}$.

The temperature dependence shows that the dominant contribution at low temperature is resonant inelastic tunneling through impurity levels. In section 3.3.2, we have shown that there is also a direct tunneling contribution to the conductivity, as evidenced by the appearance of the phonon DOS in the tunneling spectrum. This suggests that there are two parallel channels of conductivity, with the inelastic contribution dominating, partly explaining the relative weak phonon signal in figure $3.5(\mathrm{c}-\mathrm{d})$.

\subsubsection{Absence of a Josephson contact}

At this point we can address the question why we do not observe a Josephson supercurrent for the LSCO/NCCO $p n$-junctions. First, we compare the coherence length in the barrier region with the width of the barrier we get from the WKB tunneling model. The tunneling contact can be divided into two channels, a

\footnotetext{
${ }^{\dagger}$ For the $p n$ and the $p p^{\prime} n$ configuration $d I / d V(0)$ was measured directly using lock-in amplifiers; $d I / d V(0)$ for the $p n^{\prime} n$ configuration was derived numerically from temperature dependent $I V$ measurements.
} 
(a)

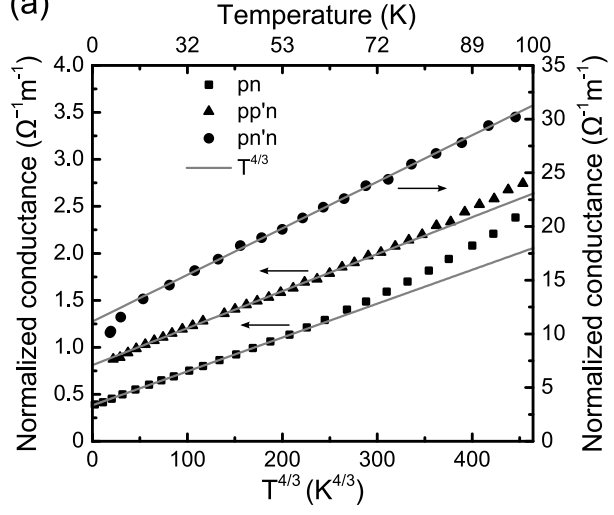

(b)

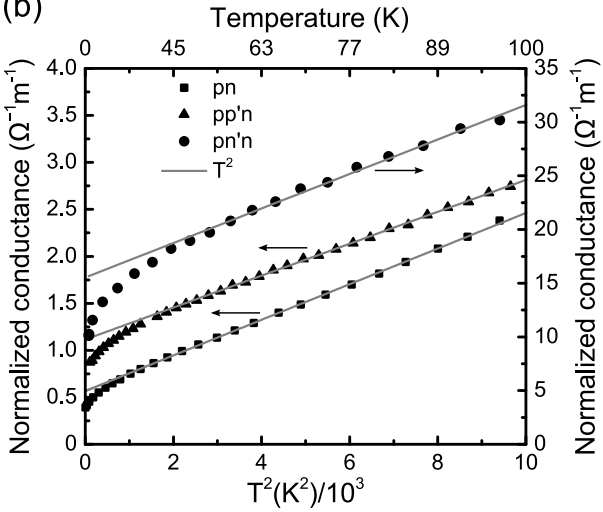

Figure 3.11: Temperature dependence of the conductance at zero bias normalized to the junction width for the $p n$ (squares), $p p^{\prime} n$ (triangles) and $p n^{\prime} n$ (circles) configuration. (a) Conductance versus $T^{4 / 3}$ following the GlazmanMatveev theory [131], indicated by the linear fit (gray lines). Note the different conductance scale for the $p n^{\prime} n$ configuration. (b) Conductance versus temperature squared, a linear fit (gray line) is added.

ballistic channel, that is responsible for the phonon spectra observed in $\S 3.3 .2$ and a channel dominated by inelastic transport giving rise to the temperature dependence seen in $\S 3.3 .7$. For the first channel we can use the coherence length in the ballistic limit $\xi=\hbar v_{F} / 2 \pi k_{B} T$, where $v_{F}$ is the Fermi velocity, and for the second channel we use the coherence length for the dirty limit $\xi=\sqrt{\hbar D / 2 \pi k_{B} T}$, where $D=v_{F} l_{e} / 3$ and $l_{e}$ is the electron mean free path [133]. The Fermi velocity in LSCO is $2.7 \times 10^{5} \mathrm{~ms}^{-1}$ [134], which is only weakly dependent on doping level, so we can take this as an estimate for the depleted region. For the electron mean free path we take $1-2 \mathrm{~nm}$, since the barrier width is around $5 \mathrm{~nm}$ and transport is dominated by two-impurity scattering. This yields a coherence length of $\sim 80 \mathrm{~nm}$ in the clean limit and 5-7 $\mathrm{nm}$ in the dirty limit. Both are the size of the barrier width or larger, so there is no a priori reason to assume there will be no Josephson contact by just looking at the coherence length.

When we take the smallest gap of the system, $3 \mathrm{meV}$ for NCCO, and assume that $I_{c} R_{N}$ is an order of magnitude smaller than the gap, similar to what we observe for the LSCO/LSCO and NCCO/NCCO junctions, we get an expected critical current per unit width for the LSCO/NCCO $p n$-junctions of $120 \mathrm{\mu} \mathrm{A} \mathrm{m}^{-1}$ (using a zero bias resistivity of $2.5 \Omega \mathrm{m}$ from figure $3.5(\mathrm{c})$ ). This results in a critical current of $20 \mathrm{nA}$ for a junction width of $150 \mu \mathrm{m}$. In order for a Josephson current to be observed, the Josephson coupling energy $\left(\Phi_{0} I_{c} / 2 \pi\right)$ has to be larger than the thermal fluctuations $\left(k_{B} T\right)$. This give a lower bound to the critical current of $\sim 180 \mathrm{nA}$ at $4.2 \mathrm{~K}$ and $\sim 50 \mathrm{nA}$ at $1.3 \mathrm{~K}$ when a zero resistance state can still be observed. Therefore, with the junction resistance of the LSCO/NCCO $p n$ junctions, we do not expect to be able to observe a clear critical current, only coherence effects around $I_{c}$. Due to the high intrinsic resistance of the junction 
combined with high contact resistances, in particular to the LSCO, the smallest current steps are limited to $10 \mathrm{nA}$ in the current measurement setup. This makes the critical current scale predicted above not observable in our experiments. We do note that we only observe small features in the $d V / d I$ characteristics of the widest junctions at currents of a few $100 \mathrm{nA}$ and a resistance that peaks sharply around zero bias with no indication of a zero bias conductance peak or onset of a supercurrent.

\subsubsection{Effect of different interlayers}

We will discuss three different scenarios for the effect of the interlayer. The first is related to the band bending in the degenerate semiconductor model, the second is related to the mixed-carrier nature of the overdoped cuprates [135], and the last concerns strain in the LSCO layer.

The first scenario is a fully electronic effect that focuses on the band bending discussed before. Here, we again assume that most of the band bending takes place in the NCCO layer. The addition of an overdoped $\mathrm{Nd}_{2-\mathrm{x}} \mathrm{Ce}_{\mathrm{x}} \mathrm{CuO}_{4}$ layer creates a larger Fermi level mismatch [85]. This will lead to a higher barrier, but with a smaller width, due to the higher carrier density associated with the overdoped phase. The situation might even change more, since the antiferromagnetic phase is less developed for overdoping. This is reflected in the barrier parameters we find for the $p n^{\prime} n$ scenario, where not only the width is smaller, but also the barrier height has decreased. An overdoped $\mathrm{La}_{2-\mathrm{x}} \mathrm{Sr}_{\mathrm{x}} \mathrm{CuO}_{4}$ layer on the other hand does not change the width of the barrier, since there is already a negligible depletion zone in the LSCO electrode. It is accompanied by a larger Fermi level mismatch [79]. This explains the scalability of the $I V$ curves for the $p n$ and the $p p^{\prime} n$-junction, as only the height of the barrier changes. From the MIM model we indeed get a higher barrier, which would correspond to the larger Fermi level mismatch, but we also find a smaller width. This might be related to some depletion effects in the LSCO that are suppressed by the higher carrier density of the overdoped phase.

For the second scenario, we note that $\mathrm{Nd}_{1.8} \mathrm{Ce}_{0.2} \mathrm{CuO}_{4}$ has a mixed, dominantly hole carrier type, as seen in chapter 2, while $\mathrm{La}_{1.75} \mathrm{Sr}_{0.25} \mathrm{CuO}_{4}$ is still hole doped; this is confirmed by Hall measurements on a single $\mathrm{La}_{1.75} \mathrm{Sr}_{0.25} \mathrm{CuO}_{4}$ film, which shows only hole type carriers down to $2 \mathrm{~K}$ and up to $9 \mathrm{~T}$. In $\mathrm{La}_{2-\mathrm{x}} \mathrm{Sr}_{\mathrm{x}} \mathrm{CuO}_{4}$ the cross-over occurs at $x>0.3$, where the Hall coefficient is negative at the lowest temperatures only for even higher overdoping [136]. Here we have to keep in mind that the degenerate semiconductor model is an approximation that neglects the strong interactions and the Mott insulator ground state that are the driving force behind the rich phase diagram of the cuprates. It is possible that the interlayers can act as mediating layer between the electron-like carriers on one side and the holelike carriers on the other side. The mixed-carrier nature of the $\mathrm{Nd}_{1.8} \mathrm{Ce}_{0.2} \mathrm{CuO}_{4}$ is best suited to act as this mediating layer, explaining the lower resistance of the $p n^{\prime} n$ contact.

In this model, the dominantly hole-like $\mathrm{La}_{1.75} \mathrm{Sr}_{0.25} \mathrm{CuO}_{4}$ layer in the $p p^{\prime} n$ configuration cannot act as a mediating layer and only adds a series resistance. When 
Table 3.1: Bulk lattice parameters for the materials discussed in this chapter.

\begin{tabular}{cccc}
\hline material & $c$-axis $(\AA)$ & $(a, b)$-axis $(\AA)$ & reference \\
\hline $\mathrm{Nd}_{1.85} \mathrm{Ce}_{0.15} \mathrm{CuO}_{4}$ & 12.08 & 3.949 & {$[38]$} \\
$\mathrm{Nd}_{1.8} \mathrm{Ce}_{0.2} \mathrm{CuO}_{4}$ & $12.025-30$ & $3.940-3$ & {$[138,139]$} \\
$\mathrm{La}_{1.85} \mathrm{Sr}_{0.15} \mathrm{CuO}_{4}$ & 13.227 & 3.775 & {$[71]$} \\
$\mathrm{La}_{1.75} \mathrm{Sr}_{0.25} \mathrm{CuO}_{4}$ & 13.223 & 3.761 & {$[140]$} \\
\hline
\end{tabular}

we assume that the $p p^{\prime} n$ configuration is simply the $p n$ configuration with a series resistance we get a resistivity of the barrier layer larger than $500 \mathrm{~m} \Omega \mathrm{cm}$. This is unrealistic for the metallic $\mathrm{La}_{1.75} \mathrm{Sr}_{0.25} \mathrm{CuO}_{4}$, which we have measured to have a resistivity of $\sim 100 \mu \Omega \mathrm{cm}$ (below $5 \mathrm{~K}$, measured for a $150 \mathrm{~nm}$ film on LSAT).

The $p n^{\prime} n$ result cannot be reproduced by simply taking the $p n I V$ characteristic and assuming an ohmic parallel channel. When the resulting $I V$ characteristic approaches the energy scale seen in the $p n^{\prime} n$ result, the low bias transport would be dominated by the ohmic channel, leading to a much lower differential resistance than is observed for the $p n^{\prime} n$ configuration. On top of this we find that a single film of $\mathrm{Nd}_{1.8} \mathrm{Ce}_{0.2} \mathrm{CuO}_{4}$ does not show any signature of electron-like carriers and has a hole-like Hall coefficient down to $2 \mathrm{~K}$.

The last scenario is a structural effect. Part of the difference between the $p n, p p^{\prime} n$ and $p n^{\prime} n$ configurations can be explained by two effects. Firstly, a restoring effect of the growth of near lattice-matched $\mathrm{Nd}_{1.8} \mathrm{Ce}_{0.2} \mathrm{CuO}_{4}$ on the NCCO electrode before the deposition of the LSCO electrode, that can recrystallize some of the damaged areas created by the argon ion milling [137]. Secondly, the strength of the strain in the LSCO layer, i.e. the lattice mismatch between the two or three layers of the configuration. The interlayer of $\mathrm{Nd}_{1.8} \mathrm{Ce}_{0.2} \mathrm{CuO}_{4}$ has a smaller in-plane lattice parameter than $\mathrm{Nd}_{1.85} \mathrm{Ce}_{0.15} \mathrm{CuO}_{4}$ (see table 3.1), resulting in a better lattice match with $\mathrm{La}_{1.85} \mathrm{Sr}_{0.15} \mathrm{CuO}_{4} \cdot \mathrm{La}_{1.75} \mathrm{Sr}_{0.25} \mathrm{CuO}_{4}$ has a smaller inplane lattice parameter than $\mathrm{La}_{1.85} \mathrm{Sr}_{0.15} \mathrm{CuO}_{4}$ (see table 3.1), resulting in a worse lattice match with the underlying $\mathrm{Nd}_{1.85} \mathrm{Ce}_{0.15} \mathrm{CuO}_{4}$ layer. When we assume that tensile strain in the LSCO layer results in a dead layer, we see that this results in a larger effect in the $p p^{\prime} n$ configuration and a smaller effect in the $p n^{\prime} n$ layer, explaining the difference in the $I V$ characteristics. The dead layer cannot merely be a bad conductor, but has to be insulating in order to explain the large resistances measured for the different configurations and to explain the non-linear behavior of the junctions, without invoking extra $p n$-physics. 


\subsubsection{Disentangling strain and electronic effects, pt. II}

A way of distinguishing the structural and electronic effects would be to reverse the layer order. In a first approximation, the Mott depletion effect should not change, but the strain effect is now in a different material. This might give a different result, but, as we have seen from bilayer experiments, the intrinsic properties of the two materials are also modified when the layer order is reversed (see chapter 5). This can potentially obscure any distinguishing effects from either of the two theories. A better way would be a careful selection of two overdoped or underdoped materials such that the strain and (Mott) depletion work in opposite directions. In this case one has to make sure that the depletion effect on the strained layer is away from superconductivity, e.g. overdoped, non-superconducting NCCO combined with underdoped, non-superconducting LSCO. In this scenario, Mott depletion would predict that the NCCO layer will traverse the superconducting dome at the interface, whereas the strain theory would only predict that the LSCO becomes more insulating at the interface. This experiment would ideally be performed with an overdoped NCCO target with extra copper added. Currently such a target is only available to us for optimal doping. Targets with higher doping levels become increasingly more difficult to fabricate due to the Ce-content being close to the solubility limit. Doing the experiment with a target without extra copper added is not recommended, as the oxygen annealing needed for the LSCO layer has a large effect on the NCCO layer when grown without extra copper, as shown in chapter 2. This can fully suppress any effect due to strain or due to the underlying Mott physics.

\subsection{Conclusions}

From the experiments on the LSCO/NCCO $p n$-contact and the addition of different interlayers we find that the energy and length scales associated with the barrier correspond best to those expected for either a strain induced effect in the LSCO or an electronic depletion effect in the NCCO. The latter is the more likely scenario when looking at the specific asymmetry of the barrier profile, which is found to be lower on the NCCO side of the junction. The $I V$ characteristics of the junctions can be well described using a metal-insulator-metal model. From this we have no direct evidence of a changing width of the depletion zone with applied bias voltage, something that would be expected from a general pn-contact.

We expect that the nature of the barrier at the interface is a combination of both the strain and the electronic effects, with strain playing a role in the LSCO layer near the interface and electronic depletion dominating on the NCCO side of the interface. An experiment with the layer order reversed could give more information, since then both effects are expected to take place in the NCCO layer.

It is interesting to note here that experiments with $\mathrm{YBCO} / \mathrm{NCCO}$ [55] do show a Josephson supercurrent and less signature of the underlying $p n$-nature of the 
interface. There is not much difference in terms of the band gap and work function between YBCO and LSCO. The main difference is found in the crystal structure of $\mathrm{YBCO}$, which has two closely space $\mathrm{CuO}_{2}$ planes and the conductivity is generally more three dimensional in nature compared to LSCO. Furthermore, the combination $\mathrm{YBCO} / \mathrm{NCCO}$ has a lower lattice mismatch than the LSCO/NCCO combination. These differences can influence both the band alignment at the interface and the size of the strain relaxation layer in the top layer.

From the energy scale of the model fits, we expect that band alignment is dictated by a hybridization of the valence band and not by the work functions of the two materials. This means that the interface states predicted by Charlebois et al. [12] are not likely to appear, since they require large band off-sets. We do see some evidence of interface or impurity states in the temperature dependence of the junctions, which can be described with the resonant inelastic tunneling model of Glazman and Matveev [131].

We propose an experiment using an overdoped $\mathrm{Nd}_{2-\mathrm{x}} \mathrm{Ce}_{\mathrm{x}} \mathrm{CuO}_{4}$ layer combined with an underdoped $\mathrm{La}_{2-\mathrm{x}} \mathrm{Sr}_{\mathrm{x}} \mathrm{CuO}_{4}$ layer to further explore the Mott nature of the depletion zone. If the depletion zone truly crosses the Mott insulator ground state, the NCCO should traverse the phase diagram of figure 1.2 in chapter 1 going towards the interface, showing a thin superconducting layer.

Finally, our results show that in our particular configuration the depletion zone is very similar to that expected in a degenerate semiconductor model. We can reach energy scales that were unobtainable in previous $\mathrm{YBCO} / \mathrm{NCCO}$ experiments [55] and see no evidence for Esaki diode behavior as was predicted by Mannhart et al. [10]. 


\section{Appendices}

\section{A Superconducting gap from phonon spectrum}

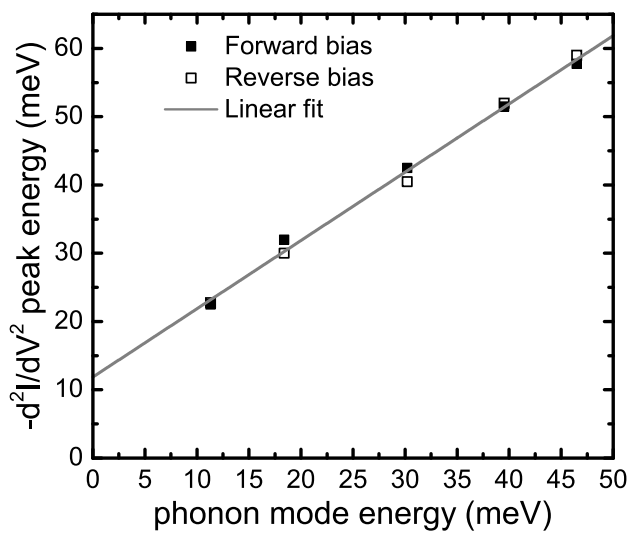

Figure A.1: $-d^{2} I / d V^{2}$ peak positions versus the corresponding LSCO phonon density of states peak positions with a linear least squares fit with slope 1 to determine the superconducting gap size. From the $y$-axis cut-off the sum of the superconducting gaps is determined to be $11.9(3) \mathrm{meV}$.

\section{B Fabrication and characterization of homojunctions}

For the LSCO/LSCO ramp-edge junctions, both layers are deposited at $700{ }^{\circ} \mathrm{C}$. The first layer is annealed after deposition using the standard oxygen annealing. After defining the ramp in the first layer, the sample is warmed up again in deposition pressure and the second layer is deposited. Again the sample is annealed using the standard oxygen annealing procedure. The NCCO/NCCO ramp-edge junctions are fabricated in a similar way, but here the deposition is done at $820^{\circ} \mathrm{C}$. After defining the ramp in the first layer, the sample is warmed up in 0.25 mbar for the deposition of the second layer. After each deposition the sample is annealed in vacuum for $8 \mathrm{~min}$ in vacuum at $740^{\circ} \mathrm{C}$, before cooling down to room temperature in vacuum. A NCCO/NCCO ramp-edge device where the first layer was warmed up in vacuum for the deposition of the second layer did not yield a Josephson contact. The sample with a Josephson contact still has an $I_{c} R_{N}$ product that is two orders of magnitude smaller than the gap. This suggests that the quality of the contact can still be improved, e.g. by warming up in a lower oxygen pressure to preserve the reduction state of the first layer or by exploring lowering of the deposition temperature of the second layer to avoid reheating the first layer to its original deposition temperature.

Figure B.1 shows resistance versus temperature measurements for LSCO/LSCO (a) and $\mathrm{NCCO} / \mathrm{NCCO}$ (b) junctions. In both cases we find the highest $T_{c}$ for the 

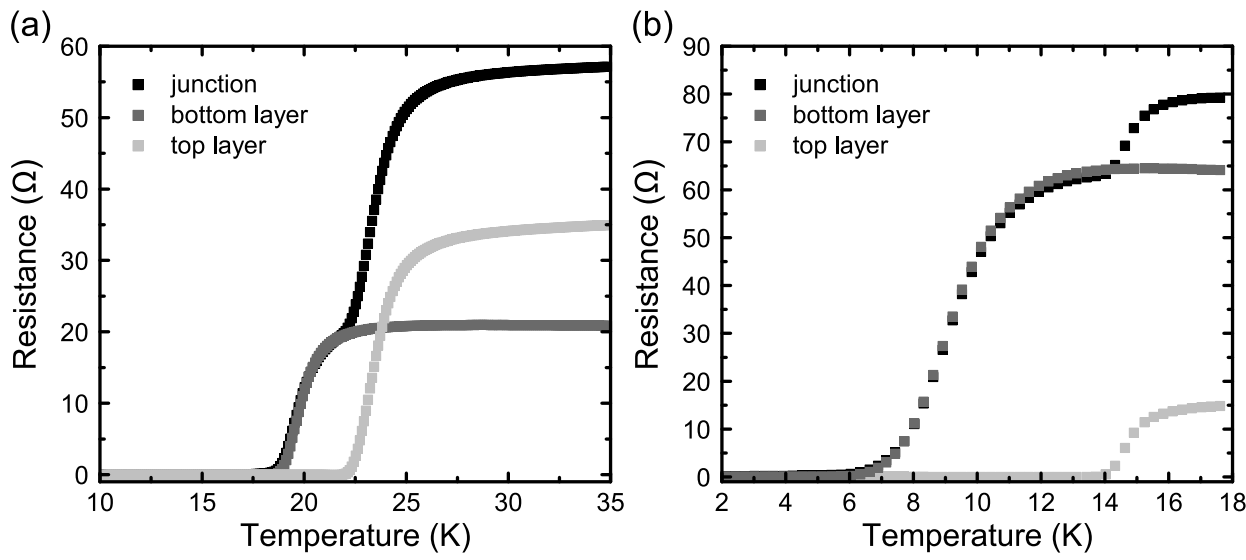

Figure B.1: Resistance versus temperature measurements for the bottom layer (dark gray), top layer (light gray) and the junction + leads (black) of the LSCO/LSCO (a) and NCCO/NCCO (b) ramp-edge homojunctions. Measured on $50 \mu \mathrm{m}$ wide junctions with a bias current of $10 \mu \mathrm{A}$.

top layer.

For the LSCO/LSCO ramp-edge junctions we find a critical current density of $8.4 \times 10^{8} \mathrm{~A} \mathrm{~m}^{-2}$ for the bottom layer and $1 \times 10^{9} \mathrm{~A} \mathrm{~m}^{-2}$ for the top layer. Even though the second layer is grown on an etched surface, it has a higher critical current. We attribute the difference between the two layers to damage or oxygen deficiency in the first layer created during thermal cycling and better pinning in the second layer due to intrinsic defects.

For the $\mathrm{NCCO} / \mathrm{NCCO}$ ramp-edge junction we see the same behavior with the first layer having a critical current density of $7.4 \times 10^{7} \mathrm{~A} \mathrm{~m}^{-2}$ and the second layer $2.7 \times 10^{8} \mathrm{~A} \mathrm{~m}^{-2}$. Here, the bottom layer is not optimally annealed due to the deposition of the second layer.

In relation to the $\mathrm{LSCO} / \mathrm{NCCO} p n$-junctions we find a higher critical current for the LSCO layers and a lower critical current for the NCCO layers for the homojunctions. The higher critical current for the LSCO layer can be explained by a better lattice match with the $\mathrm{La}_{2} \mathrm{CuO}_{4}$ buffer layer in the LSCO/LSCO junctions, as compared to the $\mathrm{Nd}_{2} \mathrm{CuO}_{4}$ buffer layer in the LSCO/NCCO junctions. The NCCO layer in the LSCO/NCCO junctions has a higher critical current because it is not heated repeatedly to high temperatures and not grown on an etched surface, where the latter gives the smallest effect.

\section{LSCO on an etched substrate}

Figure C. 1 shows resistance measurements on a $50 \mathrm{~nm} \mathrm{La}{ }_{1.85} \mathrm{Sr}_{0.15} \mathrm{CuO}_{4}$ film on an LSAT substrate that has been divided into four quadrants with different argon ion milling history. The resistance is measured in a four probe configuration with all 


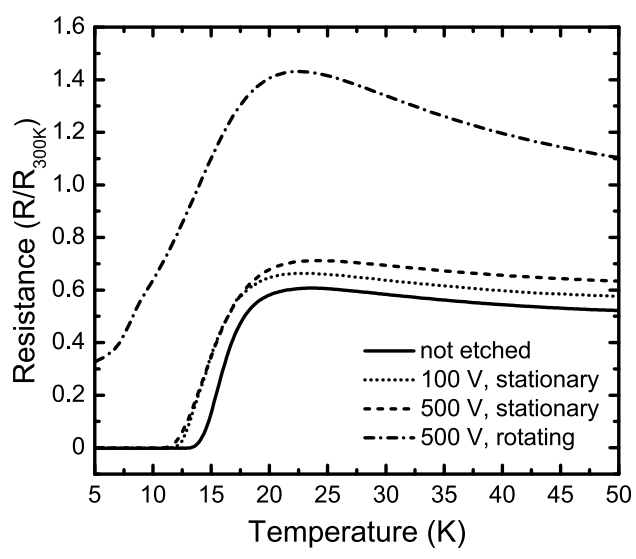

Figure C.1: LSCO grown on an argon etched LSAT substrate, all argon etches are performed under a $45^{\circ}$ angle. The substrate is divided in four quadrants with a different etching history: (1) not etched for reference (solid line), (2) 15 pulses at $100 \mathrm{~V}$, stationary (dotted line), (3) 15 pulses at $500 \mathrm{~V}$, stationary (dashed line), and (4) 15 pulses at $500 \mathrm{~V}$, rotating (dash-dotted line).

the probes in line. Therefore the resistance is normalized to the resistance at $295 \mathrm{~K}$ to allow for a more meaningful comparison. The substrate is annealed using our standard procedure for LSAT. One quadrant is not etched for reference (solid line), two quadrants are etched under $45^{\circ}$ with a $100 \mathrm{~V}$ (dotted line) and a $500 \mathrm{~V}$ (dashed line) acceleration voltage, respectively, and one quadrant is etched under $45^{\circ}$ with a $500 \mathrm{~V}$ acceleration voltage while the sample is rotating (dash-dotted line). All etched quadrants are etched for 15 pulses. The etching procedures are shown to have an effect on the resistance and the $T_{c}$ of the LSCO film. The quadrants that have been etched while the sample is not rotating both show an increase in the resistance and $T_{c}$ drops by $1.5 \mathrm{~K}$. The quadrant that was rotated during etching shows a very broad superconducting transition and never becomes fully superconducting. The resistance shows a big upturn for decreasing temperature and the general shape is very different from the reference curve of the non-etched quadrant.

$T_{c}$ is intrinsically reduced for all quadrants due to the finite thickness of the LSCO film and the use of a degraded LSCO target. The experiments discussed in this chapter were done for thicker layers and using a new target. 
CHAPTER 4

\section{Artificial domain boundary in LSCO/NCCO ramp-edge $p n$-junctions}

Scanning nano-focused X-ray diffraction (nXRD) is used to investigate the crystal structure of a ramp-edge junction between superconducting electron-doped $\mathrm{Nd}_{1.85} \mathrm{Ce}_{0.15} \mathrm{CuO}_{4}$ and superconducting hole-doped $\mathrm{La}_{1.85} \mathrm{Sr}_{0.15} \mathrm{CuO}_{4}$, the latter being the top layer. On the ramp, a three degree tilt of the LSCO $c$-axis towards the top ramp is found. This is confirmed by high-angle annular dark-field scanning transmission electron microscopy (HAADF-STEM). The tilt is explained using a strain accommodation model that relies of facet matching, dictated by the ramp angle, indicating that a coherent domain boundary is formed at the interface.

\subsection{Introduction}

Oxide heterostructures are generally characterized structurally by X-ray diffraction (XRD), transmission electron microscopy (TEM) and scanning probe techniques like atomic force microscopy (AFM), scanning tunneling microscopy (STM), piezo force microscopy (PFM), etc. Each technique has its own advantages and disadvantages. XRD is a useful tool to measure structural properties of buried layers, but generally lacks spatial resolution; scanning probe techniques have the spatial resolution, but are only surface sensitive; high resolution TEM has both spatial resolution and the ability to measure buried layers, but the measurement is very local and sample preparation by e.g. polishing and focused ion beam (FIB) can be time consuming and is destructive. Additionally there is a chance of unwanted phases forming during the ion milling.

Recent advances in synchrotron technology have led to sources with a higher brilliance and a narrower beam focus [141]. Micro and nano-focused beams have proven to be powerful tools for selective analysis, combining high lateral resolution with high $k$-space resolution [142-150]. In this work we use scanning nano-focused X-ray diffraction (nXRD) to map the composition and crystal structure of a ramp-edge junction of high- $T_{c}$ superconductors and perform a detailed analysis 
of the ramp area ( $\sim 300 \mathrm{~nm}$ wide). We then compare our findings to high-angle annular dark-field scanning transmission electron microscopy (HAADF-STEM) measurements. Using both techniques, we find a $3^{\circ}$ tilt in the LSCO crystal lattice on the ramp-edge. We explain this tilt with a model for strain accommodation by facet matching at the ramp interface. We show that nXRD can be used as a complementary, initial characterization tool that is capable of quickly measuring relatively larger areas and that is non-destructive to the sample under investigation.

\subsection{Experimental details}

\subsubsection{Sample fabrication}

The samples are fabricated by pulsed laser deposition (PLD) using both commercial and homemade polycrystalline targets. A schematic view of the junction cross-section is shown in figure 4.1(a). The ramp-edge junction starts with a bottom electrode of $\mathrm{Nd}_{1.85} \mathrm{Ce}_{0.15} \mathrm{CuO}_{4}$ (NCCO) on top of a buffer layer of undoped $\mathrm{Nd}_{2} \mathrm{CuO}_{4}$ (NCO) grown on LSAT substrates. The buffer layer is necessary to ensure the crystal quality of the second electrode in the ramp-edge junction, see chapter 3. The NCCO layer is also capped with a layer of NCO to prevent a $c$-axis contact between the top and bottom electrode in the electrical measurements. NCCO is grown from a target with extra copper added to suppress a parasitic $(\mathrm{Nd}, \mathrm{Ce})_{2} \mathrm{O}_{3}(\mathrm{CNO})$ phase [151]. A ramp is created in the multilayer stack by standard photolithography and argon ion milling under an angle of $45^{\circ}$. This step also defines the bottom electrode, see figure 4.2(c) for the electrode layout. After an in situ cleaning step of hard and soft ion milling, the top electrode of $\mathrm{La}_{1.85} \mathrm{Sr}_{0.15} \mathrm{CuO}_{4}$ (LSCO) is deposited ${ }^{\dagger}$. For HAADF-STEM, the bottom layer is structured into $100 \mathrm{\mu m}$ wide lines and the top layer is not structured to allow for easy sample preparation using focused ion beam (FIB). The sample used for nXRD is further structured into multiple devices by photolithography and ion milling. Sputter deposited $\mathrm{Au} / \mathrm{Ti}$ contacts are added as the electrodes. The outline of a typical device is depicted in figure 4.2(c). These devices, and other similar devices have been characterized electronically, see chapter 3. More details on the sample fabrication can also be found in this chapter.

\subsubsection{HAADF-STEM}

For HAADF-STEM sample preparation, the sample is covered with a thin amorphous carbon layer, an electron-beam deposited $\mathrm{Pt}$ layer and an ion-beam deposited Pt layer. A $60-100 \mathrm{~nm}$ thin lamella is defined in the ramp structure using

\footnotetext{
$\dagger$ The device structure is denoted by LSCO/NCCO in the rest of this chapter; other material combinations are also represented in this way with the second material always the bottom electrode (i.e. the material with the ramp etched into it).
} 
(a)

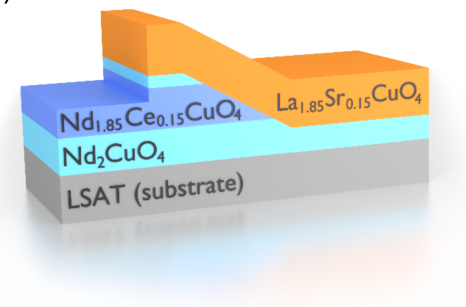

(b)

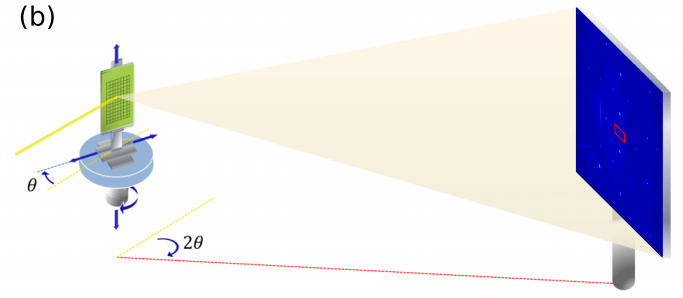

Figure 4.1: (a) Schematic cross-section of the ramp-edge junction. A ramp defined in a NCO-NCCO-NCO stack connects the NCCO bottom electrode (blue) to the LSCO top electrode (orange). (b) Schematic representation of the nXRD setup at the ID13 beamline of the European Synchrotron Radiation Facility (ESRF). The sample can be scanned in the X-ray beam using $x, y$ actuators and also rotated $(\theta)$. A 2D CCD detector collects the diffracted X-ray pattern. From [152].

FIB. The FIB sample preparation was finished with a final milling and cleaning of the surface from $5 \mathrm{kV}$ down to $2 \mathrm{kV}$, and finally $1 \mathrm{kV}$, to minimize damage and unwanted redeposition. The HAADF-STEM measurements were performed by dr. Xiaoxing Ke in the group of prof. van Tendeloo at the University of Antwerp, using a FEI Titan 80-300 "cubed" microscope fitted with an aberration-corrector for the probe forming lens and SuperX EDX detectors, operated at $200 \mathrm{kV}$. High resolution HAADF-STEM images were taken from the ramp interface of the rampedge junctions with the substrate tilted to the $\left[\begin{array}{lll}1 & 0 & 0\end{array}\right]$ zone-axis.

\subsubsection{Scanning nano-focused X-ray diffraction}

The nXRD experiments were performed at the ID13 beamline of the European Synchrotron Radiation Facility (ESRF), Grenoble, France. The ID13 nanobranch is specialized in the delivery of nano-focused X-ray beams for diffraction experiments. The photon source, an $18 \mathrm{~mm}$ period in-vacuum undulator, works in the range $5-17 \mathrm{keV}$ with the storage ring operating at $6.03 \mathrm{GeV}$ in the uniform mode with a current of $200 \mathrm{~mA}$. The beamline uses a Si-111 channel cut crystal monochromator cooled with liquid nitrogen. A monochromatic X-ray beam of photon energy $14.9 \mathrm{keV}\left(\Delta E / E=10^{-4}\right)$ was used, which was focused by Kirkpatrick-Baez (KB) mirrors to a $300 \mathrm{~nm}$ spot size on the sample (full width at half maximum). Figure 4.1(b) shows a schematic of the measurement setup. A 16-bit 2D Fast Readout Low Noise charged coupled device (FReLoN CCD) detector with $2048 \times 2048$ pixels of $51 \mu \mathrm{m} \times 51 \mu \mathrm{m}$ was used. The detector was placed $50 \mathrm{~mm}$ behind the sample and offset. Diffraction images were obtained after correcting the 2D images for dark noise, flat field and distortion. The FreLoN CCD camera records the intensity of the peak that correspond to the square root volume of the domains in the selected sample surface spot. The intensity is integrated over 
(a)

(1) NCCO

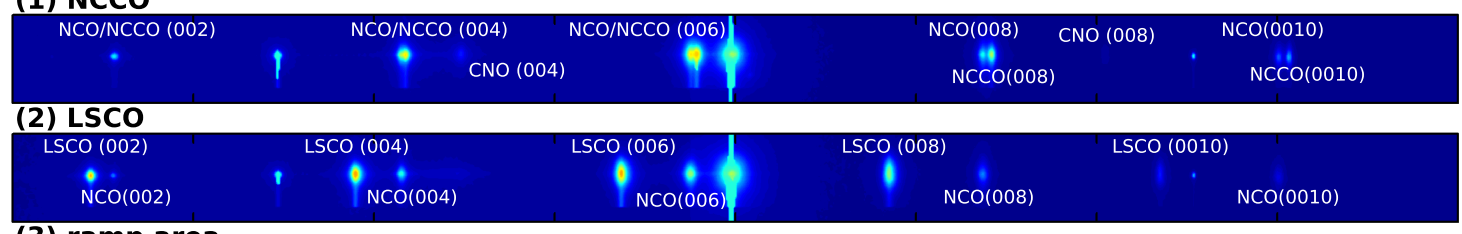

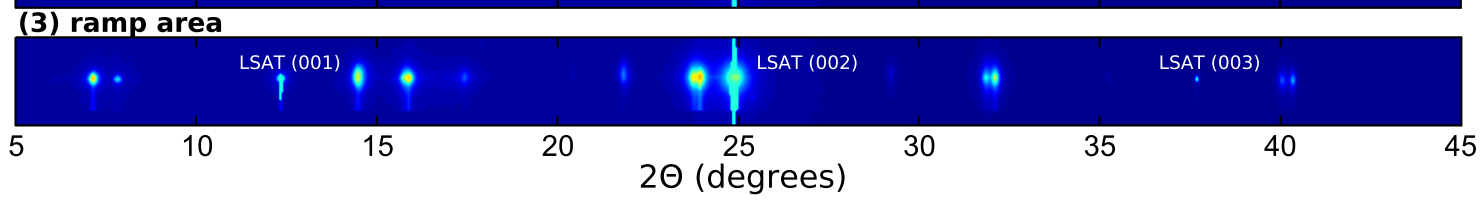
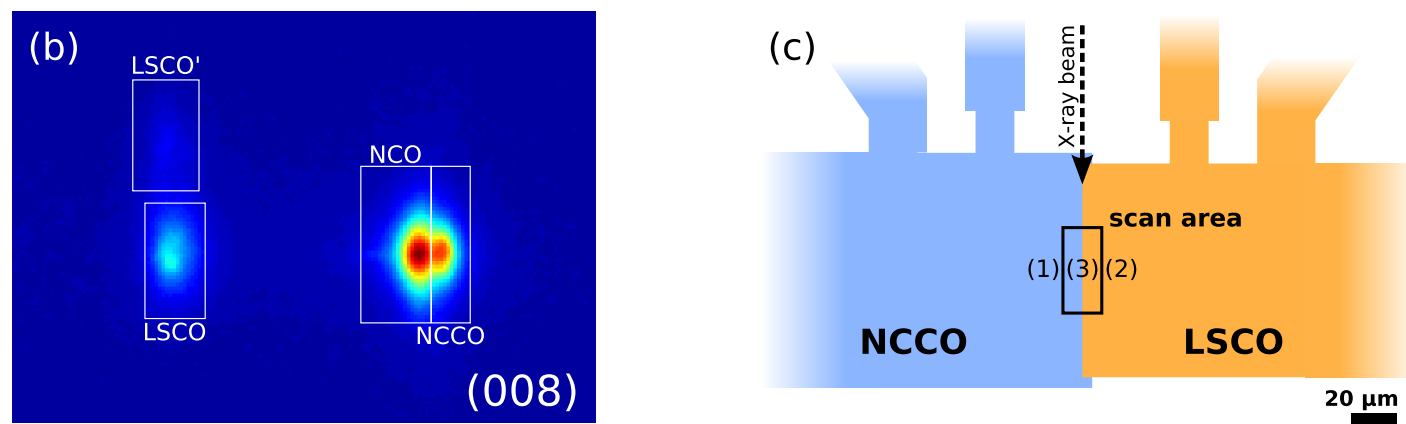

Figure 4.2: (a) $\theta-2 \theta$ nXRD scans on different parts of the device, namely the NCCO layer (1), the LSCO layer (2) and the ramp area (3). Peaks for LSCO, NCCO, NCO, $(\mathrm{Nd}, \mathrm{Ce})_{2} \mathrm{O}_{3}$ and LSAT are indicated. The color scale is logarithmic. (b) Close-up of the $\mathrm{NCO} / \mathrm{LSCO} / \mathrm{NCCO}$ (0 08 ) reflections; the area integration used for the mapping of figure 4.3 is indicated $(\mathrm{NCO}, \mathrm{NCCO}$, LSCO and $\mathrm{LSCO}^{\prime}$, a tilted phase which is connected with strain accommodation on the ramp). The color scale is logarithmic. (c) Schematic representation of the junction with the areas of (a) labeled. The black box is the scan area used for figure 4.3. The direction of the beam is indicated by the black arrow.

square subareas of the images recorded by the FreLoN CCD detector in reciprocallattice units of the main crystalline reflections at each point $(x, y)$ of the sample reached by the translator. For most of the experiments discussed here, the angle of incidence of the X-ray beam with the sample was around $16^{\circ}$. This gives an effective interaction length of $300 \mathrm{~nm}$ perpendicular to the beam and $0.8-1.3 \mu \mathrm{m}$ in the beam direction due to the finite thickness of the sample, as the thickness of the layers of interest is $150-300 \mathrm{~nm}$.

In the experiment, the device is first aligned by microscope and then $\theta-2 \theta$ scans are performed on selected areas by rotating the sample in the beam with the CCD detector stationary; for each angle step a CCD frame is recorded. The $\theta-2 \theta$ map is constructed by summing all the frames. Antisymmetric reflections are removed in the image processing by only collecting in a moving box that follows $2 \theta$. Figure 4.2 (a) shows $\theta-2 \theta$ scans (color scale is logarithmic) for the NCCO electrode (1), 
the LSCO electrode (2) and the ramp area (3). The $(00 l)$ reflections, with $l$ even, for LSAT, NCCO, NCO and LSCO are indicated. The parasitic $(\mathrm{Nd}, \mathrm{Ce})_{2} \mathrm{O}_{3}$ phase is also present in all three figures, since there is always a layer of $\mathrm{NCO}$ remaining. The $(\mathrm{Nd}, \mathrm{Ce})_{2} \mathrm{O}_{3}(004)$ and $(008)$ reflections can be identified. The scan of the junction area also shows a weak signal for gold (111), which appears as polycrystalline rings in the $\theta-2 \theta$ scan without the moving box algorithm. This is attributed to gold particles collected at the sharp edges of the ramp and the LSCO layer overlap during scanning SQUID microscopy ${ }^{\dagger}$. Here, the scanning SQUID probe was in direct contact with the sample, scraped off some of the soft gold of the measurement electrodes, and deposited it at all sharp edges of the device. This was confirmed by optical microscope. Strong film peaks and especially the LSAT (001) and (002) substrate peaks show streaks due to saturation of the CCD detector. Figure A.1 in appendix A shows a full projection corrected $\chi-2 \theta$ map with the saturation removed and showing the polycrystalline lines associated with the gold particles.

Next, the sample is aligned to NCCO (008) for the diffraction mapping. NCCO $(008)$ is selected over the higher intensity peak of $(006)$, because at this angle NCO (008) and NCCO (008) can be separately resolved, see figure 4.2(b). Furthermore, no nearby substrate peaks give a changing background due to e.g. mosaic spread in the substrate and saturation of the CCD detector. As shown in figure $4.2(\mathrm{c})$, the sample is scanned in a grid across the junction area with a step size of $1 \mu \mathrm{m}$ along the junction and $0.5 \mu \mathrm{m}$ perpendicular to the ramp with the junction interface parallel to the beam to get the highest resolution across the junction. At each pixel, a CCD image is collected; figure 4.2(b) shows a close-up around the (0 08$)$ peaks used for the nXRD mapping (color scale is logarithmic). A beam convergence, due to the focusing of the beam, enables us to measure NCCO (0 0 8), NCO (008) and LSCO (0 0 8) at the same time, allowing for a mapping of all the materials of the device. Off-axis peaks attributed to NCCO, NCO, LSCO and LSAT are also visible and available for further mapping and e.g. tilt/strain analysis.

\subsection{Results and discussion}

\subsection{1 nXRD mapping}

Figures 4.3(a-d) show a mapping of the integrated peak intensity for NCO (0 08 ), NCCO (008), LSCO (008) and a tilted LSCO (008) phase, respectively. These show an intensity mapping of the ramp area by integrating over the white boxes labeled $N C O, N C C O, L S C O$ and $L S C O^{\prime}$ in figure 4.2(b). Here, we only consider the $(008)$ reflections, as they are not sensitive to a rotation of the sample (rotation in $p h i$ ). Off-axis peaks have also been mapped and show nominally the same result.

\footnotetext{
$\dagger$ This technique was used to measure the diamagnetic response and the vortex structure of the junctions discussed in chapter 3 , but it did not yield information suitable for the discussion in chapter 3.
} 

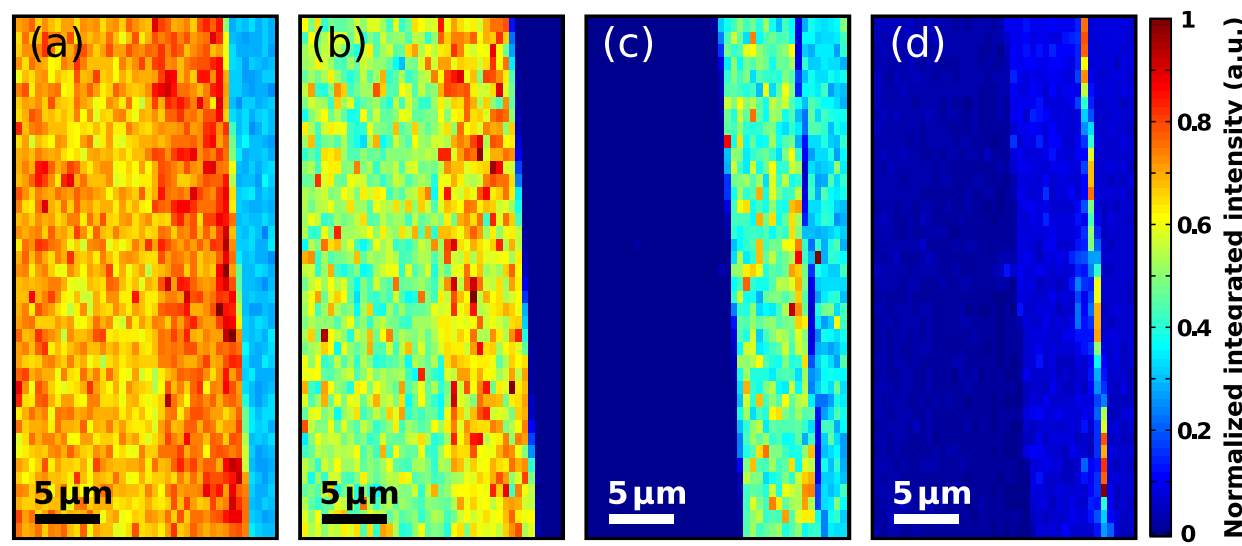

Figure 4.3: Integrated nXRD intensity maps for NCO (a), NCCO (b), LSCO (c) and a tilted phase of LSCO (d), only visible at the ramp, measured on the scan area indicated in figure 4.2(c). A background is subtracted from all maps and the signal is normalized to the highest intensity pixel. The pixel size is $0.5 \mu \mathrm{m} \times 1 \mu \mathrm{m}$.

For the mapping, a background is subtracted by using the average intensity level when no peak is present, i.e. the dark blue areas in figure 4.3(b-d). For figure 4.3(a), the background of (b) is used, scaled to the size of the integration box. Each image is then normalized to the highest intensity pixel. The pixel size is $0.5 \mu \mathrm{m} \times 1 \mu \mathrm{m}$, with the shortest size in the horizontal direction. The device architecture of figure $4.2(\mathrm{c})$ can be identified. For NCO, figure 4.3(a), going from left to right, first the NCO layer underneath the NCCO electrode is imaged. Then the junction overlap area, which has a higher intensity, because here the NCO capping layer has not been etched away. The ramp is identified as the step-like intensity change from orange to light blue in 1-2 pixels, comparable to the width of the ramp ( $\sim 300 \mathrm{~nm}$, see e.g. figure 4.1(a) or 4.5(a)). Lastly, beyond the ramp on the right, finite intensity remains as the etching process of the ramp is stopped in the NCO layer. In the mapping of NCCO, figure 4.3(b), both the overlap area and the ramp can be identified. The overlap area shows a higher intensity because the part outside the overlap area is etched away slightly during the definition of the LSCO contact and the removal of the NCO capping layer. Beyond the ramp only background intensity remains. Finally, for LSCO, figure 4.3(c), we get a complementary picture. The overlap area can be identified and no intensity remains to the left of the overlap area, where all the LSCO has been etched away. The higher intensity for the overlap area can be explained by a higher crystal quality of LSCO on the overlap as compared to the LSCO grown on the etched surface of NCO beyond the ramp. 


\subsubsection{Tilted LSCO phase}

At the position where the ramp can be identified in the NCO and NCCO maps, we observe missing intensity in the LSCO map. Further analysis of the CCD images has revealed that part of the LSCO (008) intensity shifts up on the detector. Figure 4.3(d) shows a mapping for the intensity of this shifted phase, box $L S C O^{\prime}$ in figure $4.2(\mathrm{~b})$. Here, the intensity along the ramp corresponds to the missing intensity of figure $4.3(\mathrm{c})$. The finite intensity measured outside of the ramp area is attributed to the area selection for the mapping, which also picks up intensity from the non-tilted peak in the $L S C O^{\prime}$ box. The two peaks can be better resolved for LSCO (107), see figure A.2 in appendix A. A mapping of the (107) shows little to no intensity for the shifted phase outside of the ramp area.

Figure 4.4 shows a summation (on a logarithmic scale) of all the frames collected in the mapping of figure 4.3. Here, we observe the same shift for all peaks attributed to LSCO, i.e. $(008),(107)$ and $(\overline{1} 05)^{\dagger}$. In the shift, the $2 \theta$ angle does not change, as indicated by the dotted lines of equal $2 \theta$ in figure 4.4. This excludes lattice deformation as the origin of the shift, since that would be accompanied by a change in $c$-axis length and thus a $2 \theta$ shift. We therefore attribute the shift of the LSCO peaks to a tilting of the LSCO lattice, while the LSCO unit cell remains unchanged. The tilt is measured to be around $3^{\circ}$ from the shift of the $(008)$, (107) and $(\overline{1} 05)$ peaks of LSCO, as indicated in figure 4.4. It corresponds to a tilting of the $c$-axis towards the top of the ramp.

\subsubsection{HAADF-STEM}

The nXRD results are confirmed by HAADF-STEM and energy dispersive X-ray (EDX) spectroscopy measurements. Figure 4.5(a) shows a TEM cross-section of the full device where the layers from figure 4.1(a) can be recognized. The layer composition is verified by electron diffraction and EDX spectroscopy, discussed in more detail in chapter 3 . The tilting of the LSCO $c$-axis on the ramp is indicated schematically. Figure 4.5(b) shows a close-up of the ramp area; the LSCO layer on the ramp shows a $3^{\circ}$ tilt with respect to the NCCO lattice. On either side of the ramp the LSCO layer is grown $c$-axis aligned on the NCCO layer (figure 4.5(a), left and right edge). In figure 4.5(a) the transition between the tilted phase and the $c$-axis aligned phase manifest itself as a grain boundary at the bottom of the ramp, indicated by the black arrow.

\subsubsection{Origin of the LSCO lattice tilt}

Lattice tilt is known to occur in ramp-edge structures of cuprate superconductors and it is exploited for Josephson junction fabrication [124]. In most cases however, the tilt of the layer on the ramp is away from the ramp in a growth mode

$\dagger$ A small in-plane rotation accounts for the difference in visibility of e.g. the (107) and ( 107$)$ peaks. 


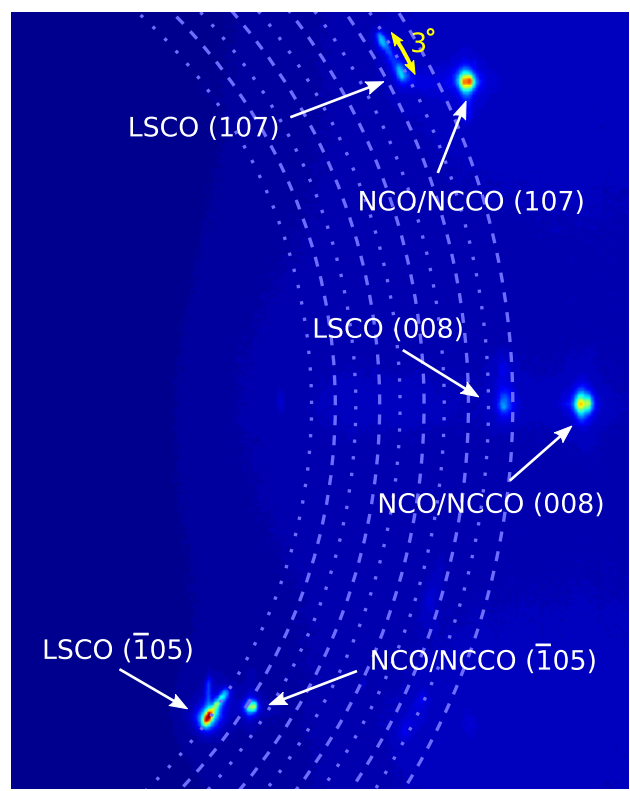

Figure 4.4: Summation of all CCD frames collected in the mapping of figure 4.3 (logarithmic color scale), showing that the shifted LSCO phase tilts without changing $2 \theta$. NCO/NCCO and LSCO (008), (107) and (105) are labeled. The dotted lines indicate equal $2 \theta$ angles.

called graphoepitaxy. Graphoepitaxy occurs for heterostructures with large lattice mismatch, where the growth of the second layer follows the surface normal instead of the crystal axis of the underlying layer. For YBCO this is seen for example when growing on a ramp etched into a $\mathrm{MgO}$ substrate; here, the YBCO $c$-axis follows the surface normal, tilting up to $45^{\circ}$, creating Josephson junctions at the top and bottom of the ramp $[124,153]$. In our case the tilt does not follow the ramp angle directly and is less dramatic than the graphoepitaxial growth. In chapter 3 we argue that the tilting of the lattice is not expected to have a major influence on the transport properties of the junction. Nonetheless, it is a curious effect that can be used to show the resolving power of the nXRD technique. This will become evident when we discuss the origin of the lattice tilt.

There are two possible origins for the three degree lattice tilt, namely a strain gradient originating from the ramp interface or strain accommodation by facet matching that induces a tilt. We will discuss both theories in more detail.

The first possibility is that the $3^{\circ}$ tilt of LSCO on the NCCO ramp is caused by a strain gradient. Lattice strain from the NCCO bottom layer will relax in the LSCO layer to a 'bulk' value far away from the interface. The angle of the ramp would also cause a lateral relaxation in the LSCO film, on top of the relaxation following the thickness of the film. Moving away from the ramp horizontally an increase in the $c$-axis length of the LSCO should be observed as the film relaxes. This changing $c$-axis length may explain the observed tilt of the lattice planes. 

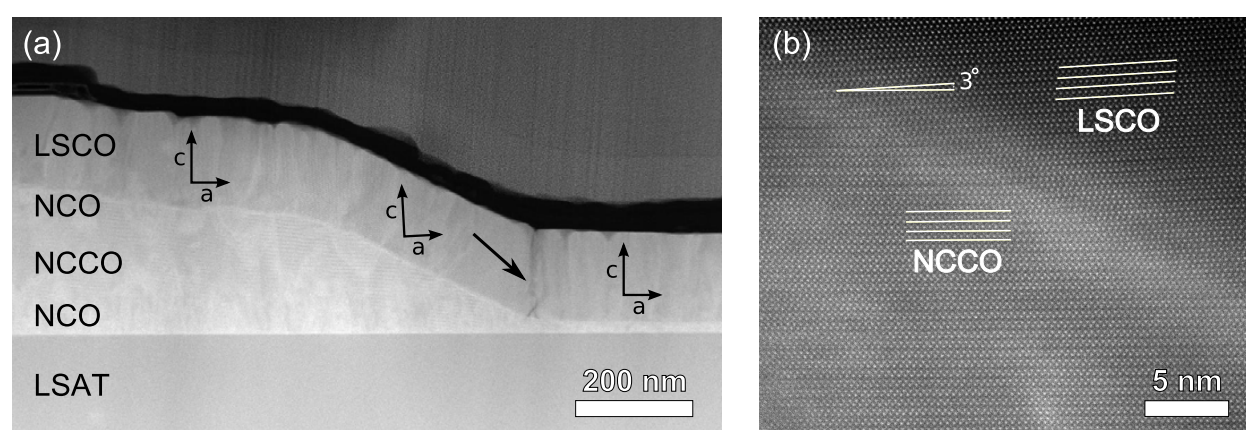

Figure 4.5: TEM cross-section of a NCCO-LSCO ramp-edge junction. (a) Overview of the junction geometry, the major layers are labeled. The arrow indicates a grain boundary between a tilted LSCO phase on the ramp and the $c$-axis aligned phase away from the ramp. (b) TEM close-up of the ramp, NCCO and LSCO are indicated. The LSCO phase shows a tilt of $3^{\circ}$ with respect to the $\mathrm{NCCO}$ lattice.

However, strain relaxation cannot fully explain the observed tilt of $3^{\circ}$. Relaxation can account for a slight bending of the lattice planes near the ramp interface, as is observed in figure 4.6. Here, a small bend of the LSCO lattice is observed due to strain near the LSCO/NCCO ramp interface, suggesting a strain relaxation length of around $5 \mathrm{~nm}$. Further away from the ramp any tilting can only be linked directly to the ramp angle. Imagine two points on the tilted LSCO lattice plane and the columns of LSCO unit cells underneath, further away from the ramp than the strain relaxation length. The length over which the strain relaxes is the same for both columns so any misalignment (which manifests itself as the tilt) can only come from the shift of LSCO unit cells, relaxed to the 'bulk' value of the rest of the film. This shift is only determined by the angle of the ramp. Any apparent tilt of the LSCO lattice in this scenario, will therefore either follow the ramp angle $\alpha$ or tilt away from the ramp with $90^{\circ}-\alpha$. Thus, we conclude that a strain gradient is not the origin of the observed lattice tilt.

A second explanation for the tilted phase is a better lattice match for the tilted phase on the NCCO facets exposed on the ramp. The tilted phase then forms as a means of strain accommodation. Tilting to accommodate lattice mismatch is observed in semiconductor heterostructures for large lattice mismatches in combinations like MnAs/GaAs, GaN/GaAs, GaAs/Si, Cu/GaAs or $\alpha-\mathrm{Si}_{3} \mathrm{~N}_{4} / \mathrm{Si}[154-157]$. Here, the tilt is determined by the lattice mismatch and the vicinal angle of the substrate. In our case the tilting is determined by the angle of the ramp. From STEM and AFM, we have a ramp angle of around $26^{\circ}$, the facet closest to this angle is NCCO (3019). The corresponding LSCO (3019) plane has an incline of about $29^{\circ}$, which gives a $3^{\circ}$ incline for the $\left(\begin{array}{lll}0 & 0 & 1\end{array}\right)$ planes, when the unit cell is tilted to make the (30 19) planes of NCCO and LSCO parallel. The lattice mismatch between LSCO (3019) and NCCO (3019) in the direction along the ramp is $1.7 \%$ versus an in-plane mismatch of $4.4 \%$ for the $c$-axis aligned growth. The (3019) plane is not the only plane with approximately the same angle as the ramp, other 


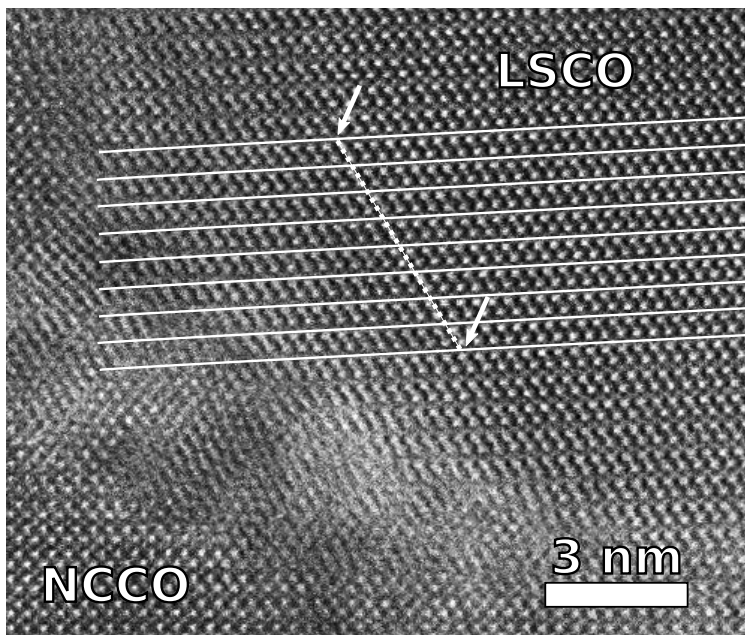

Figure 4.6: TEM image showing strain relaxation at the LSCO/NCCO interface. The white lines follow the bulk lattice planes; at the interface the lattice planes bend away from the white lines. The dotted line and the two arrows indicate where the bending starts. This image was taken on the side of the mesa that was in the direct argon ion beam during the definition of the ramp, hence the higher degree of disorder compared to the interface of figure $4.5(\mathrm{~b})$.

planes like $(106),(107),(2013)$ and $(3020)$ all have an incline close to $26^{\circ}$. For all these planes the corresponding LSCO plane is also tilted by about $3^{\circ}$ and the lattice mismatch is always less than $2 \%$.

Far away from the ramp the growth of LSCO is c-axis aligned. Where the two lattices meet, we expect a grain boundary, which would nucleate at the bottom of the ramp. This is indeed what we observe in the TEM cross-section of the ramp, see figure 4.5(a). In this scenario, a different ramp angle should promote different facets of NCCO and thus result in LSCO layers with a different tilt. In the cross-section we can see a curvature in the ramp at the bottom and at the top. At the bottom this is accompanied by a grain boundary; at the top there is no obvious grain boundary. The top of the structure is therefore the best place to for a changing tilt as function of the ramp angle. Assuming that for each angle the Miller indices of the aligned NCCO and LSCO planes are the same, the tilt angle $\gamma$ is given by

$$
\gamma=\arctan \left(\frac{a_{N} c_{L}}{c_{N} a_{L}} \tan \alpha\right)-\alpha
$$

where $\alpha$ is the ramp angle in degrees and $a(c)_{N, L}$ is the $a(c)$-axis length of NCCO $(\mathrm{N})$ and LSCO (L). A full derivation can be found in appendix B. Figure 4.7(a) schematically shows the tilting process and the blue curve (left axis) in figure 4.7(b) shows the dependence of equation (4.1). The tilt angle will peak to close 
(a)
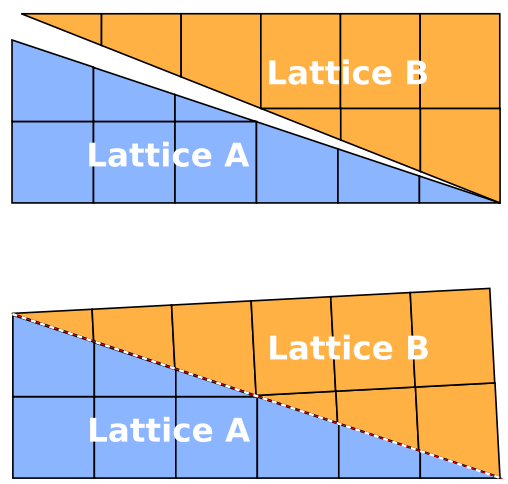

(b)

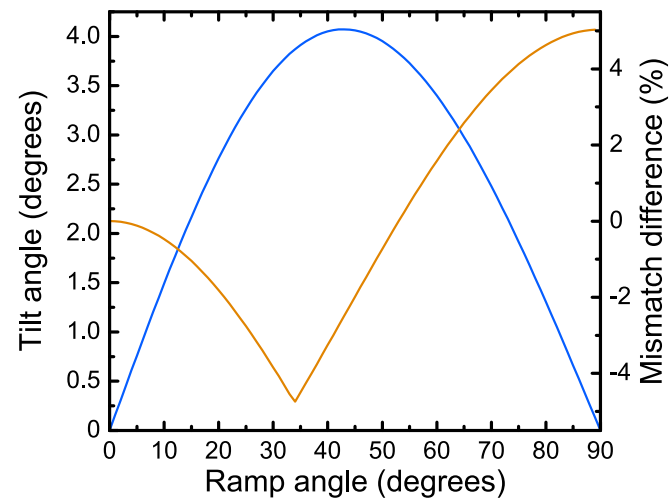

Figure 4.7: (a) Schematic representation of the tilting process for two lattices with an in-plane lattice mismatch and different $c / a$ ratios. (b) Tilt of the LSCO phase on the NCCO ramp toward the top of the ramp as a function of ramp angle, as depicted in (a), following equation (4.1) (blue curve, left axis). The other curve shows the absolute lattice mismatch difference between the tilted phase and a $c$-axis oriented phase, where a negative value indicates a lower mismatch for the tilted phase (orange curve, right axis).

to $4^{\circ}$ around a ramp angle of $43^{\circ}$ when the bulk values for the $(a, b)$ and $c$-axis for $\operatorname{NCCO}(a=3.949 \AA, c=12.08 \AA[38])$ and $\operatorname{LSCO}(a=3.7752 \AA, c=13.227 \AA[71])$ are used. The orange curve (right axis) shows the difference between the absolute lattice mismatch for the tilted phase and the in-plane absolute lattice mismatch for the non-tilted phase $\left(\left|a_{N}-a_{L}\right| / a_{N}\right)$; a negative value indicates that the tilted phase has a favorable, lower lattice mismatch. The tilted phase is predicted to be unfavorable for ramp angles higher than $52^{\circ}$.

We can also extract the ratios $a_{N} / a_{L}$ and $c_{L} / c_{N}$ in equation (4.1) from the HAADF-STEM image of figure 4.5. By concentrating on the ratios we can limit errors due to image distortion. We find $a_{N} / a_{L}=1.05$ and $c_{L} / c_{N}=1.098$, compared to 1.046 and 1.095 , respectively, when using the literature values. The tilt of the LSCO lattice can be more accurately determined by looking at the fast Fourier transform of the NCCO and the LSCO layer and is determined to be $3.3^{\circ}$. Using a ramp angle of $26^{\circ}$ in equation (4.1), we obtain a predicted tilt of $3.35^{\circ}$ when we take the measured ratios. This is within the measurement error bar, which is mostly dominated by inaccuracy in determining the ramp angle; an estimated spread of $1^{\circ}$ translates to a spread of $0.1^{\circ}$ for the predicted tilt angle, which is comparable to the size of the data point in figure 4.9.

Using equation (4.1), we can predict that the tilt will not change too much with different doping levels for NCCO and LSCO, as the ratio $a / c$ does not change appreciably as a function of doping for either material. Over a doping range of $x=0 \ldots 0.2$ for $\mathrm{Nd}_{2-\mathrm{x}} \mathrm{Ce}_{\mathrm{x}} \mathrm{CuO}_{4}$, the predicted tilt for the LSCO layer will only vary by $2 \%$. 


\subsubsection{Model validation}

Figure 4.8 visualizes the tilting of the LSCO lattice as the X-ray beam is scanned across the junction. As can be seen from the mapping in figure 4.3, the beam crosses the ramp interface at a small angle; the map is built by scanning in vertical lines from top to bottom. Panels $1-5$ in figure 4.8 show the $\left(\begin{array}{ll}1 & 07) \\ \text { ) diffraction peaks }\end{array}\right.$ for NCO, NCCO and LSCO corresponding to different areas of the ramp-edge structure. As the beam crosses the interface, the LSCO (10 7) can be seen to shift in different ways at the top and the bottom of the ramp. Panel 2 corresponds to the top of the ramp structure where there is a gradual change in the angle of the ramp, creating a spread out peak structure. On the ramp, only the tilted phase is observed (panel 3). At the bottom of the ramp, the angle changes, but not as gradual as at the top and the tilted phase meets the $c$-axis aligned phase in a grain boundary. This is reflected in panel 4 , where two distinct peaks are observed, with only small streaking between the two. Finally, in panel 5 , the LSCO is fully $c$-axis aligned again.

The ramp is not a single uniform facet, it has a certain roughness. This should give a spread in the tilt angles observed. The roughness of the ramp would give a distribution of facet angles, both bigger and smaller than the actual ramp angle. The roughness of the ramp therefore only determines the angular width of the diffraction peak of the tilted phase. The angular width of the peak corresponding to the tilted phase in figure 4.8 does not have a larger width than the peak for the $c$-axis aligned phase. This indicates that if there is an angular spread due to roughness on the ramp, it is smaller than the instrumental broadening and the finite thickness broadening of the diffraction peak.

This leads us to conclude that the facet matching model can qualitatively describe the LSCO tilt over the whole ramp structure and qualitatively predict the tilt angle for the dominant ramp angle.

\subsubsection{Other material combinations}

It is interesting to see what equation (4.1) predicts for other materials commonly used in ramp-edge junctions. Most junction designs work with YBCO, which as it turns out, has a $c / a$ ratio very comparable to the $n$-type cuprates. In the configuration of YBCO/NCCO [55] or for NCCO as interlayer in YBCO-YBCO Josephson junctions [57], the predicted tilt is small, $<0.3^{\circ}$, and it results in an unfavorable lattice mismatch compared to $c$-axis aligned growth. The same holds for other common interlayer materials like PBCO [158] and for YBCO grown on ramps etched into substrates like $\mathrm{LaAlO}_{3}$ [159] or $\mathrm{MgO}$ [124], where in the latter case graphoepitaxy is found due to the large in-plane lattice mismatch. A scenario similar to LSCO/NCCO is found in the combination of YBCO and LSCO. The theory predicts a tilt of $3.45^{\circ}$ for $\mathrm{LSCO} / \mathrm{YBCO}$ and $-3.16^{\circ}$ for $\mathrm{YBCO} / \mathrm{LSCO}$ both for a ramp angle of $26^{\circ}$. The maximum ramp angle for which a tilted phase is expected to occur is $30^{\circ}$ and $34^{\circ}$, respectively. These material combinations have been studied by the Maeda group $[160,161]$, but no reports are available. 

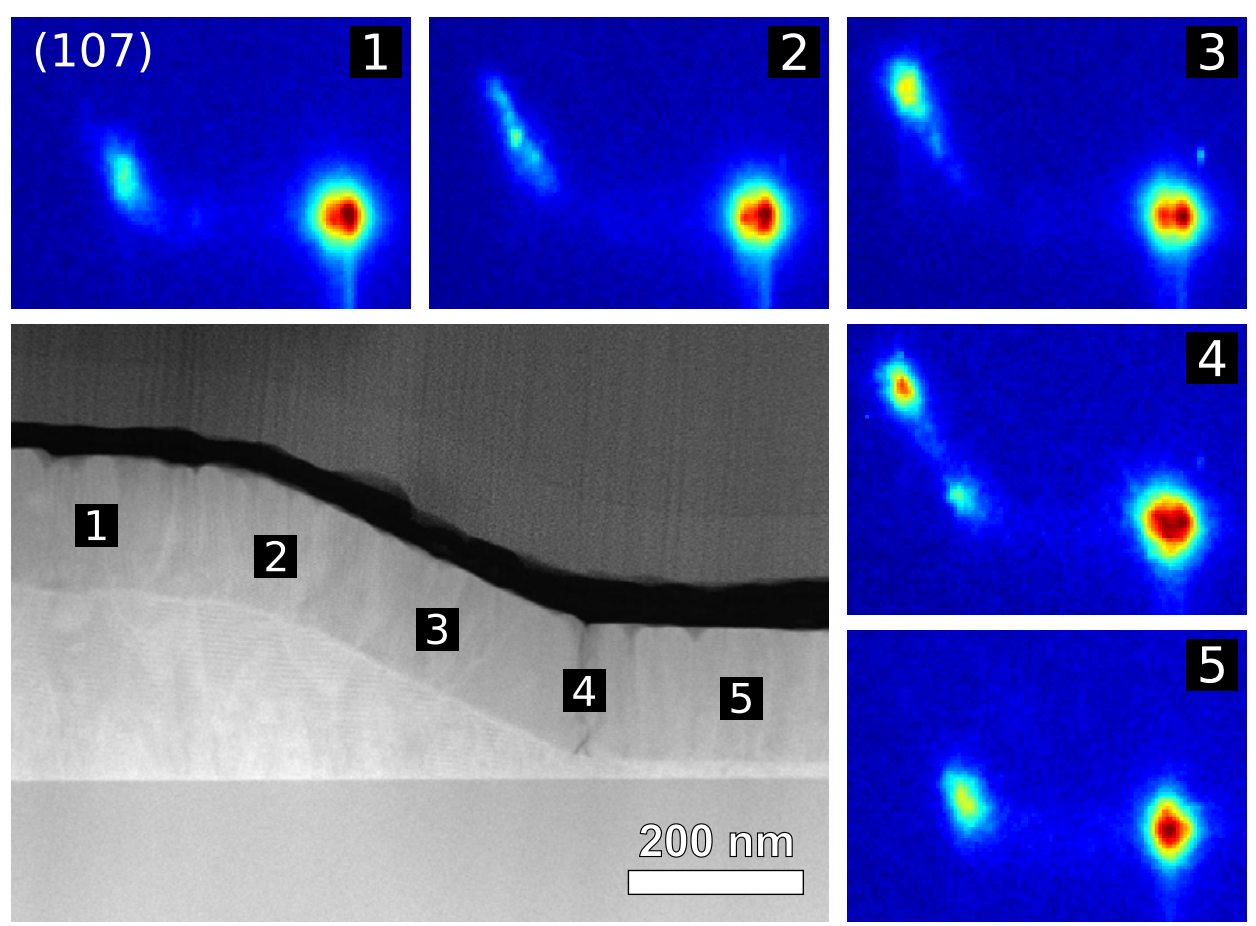

Figure 4.8: Diffraction patterns for the (107) diffraction peaks of NCO, NCCO and LSCO at different approximate positions on the junction structure $(1-5)$. The LSCO (107) can be seen to shift differently at the top and the bottom of the ramp; at the top (2) the peak is broad due to a changing ramp angle, resulting in a varying tilt for the LSCO; at the bottom the change is abrupt, with two distinct peaks due to the grain boundary between the tilted phase and the $c$-axis aligned phase of the LSCO.

An even simpler test architecture for the model is a single film grown on a substrate with different ramp structures etched into it. For enough ramp surface, the tilt of the film should be measurable using a lab source by an X-ray diffraction rocking curve as a distinct side peaks on both side of the peak for the $c$-axis oriented film grown on the flat substrate surfaces. We propose to use LSAT or STO substrates and LSCO or YBCO as the film materials. On both LSAT and STO the YBCO layer is predicted to show a tilt for all experimentally available angles increasing to a maximum of $0.26^{\circ}$ at a ramp angle of $45^{\circ}$. LSCO grown on a ramp in STO is predicted to show a tilt increasing to almost $4.5^{\circ}$ up to a ramp angle of $40^{\circ}$. On LSAT, a tilt up to $4.1^{\circ}$ is predicted up to a ramp angle of $32^{\circ}$.

Another way would be to use the current material combination of LSCO/NCCO and fabricate a structure with many different ramp angles by etching multiple bar-shaped structures using different argon ion milling angles. A structure like this can be measured quite easily with a technique like nXRD, which has sufficient resolution to measure the local tilt of the lattice. This technique is preferred over a 


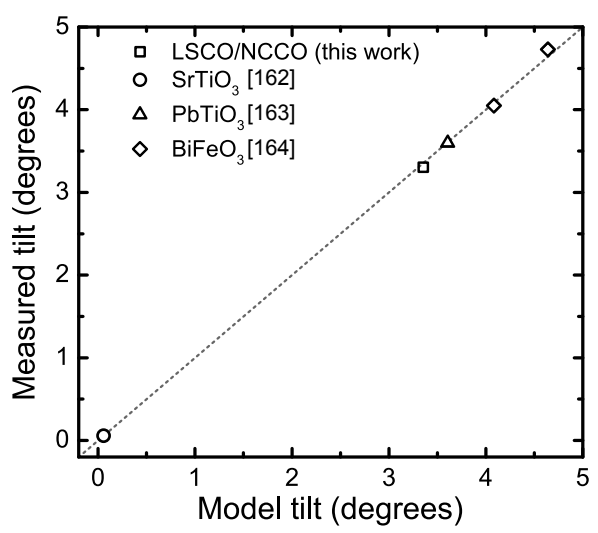

Figure 4.9: Experimental tilt versus model tilt using equation (4.1) for various oxide systems. The data points for $\mathrm{SrTiO}_{3}, \mathrm{PbTiO}_{3}$ and $\mathrm{BiFeO}_{3}$ are determined using information from the main text and the TEM measurements in references [162-164]. The dotted line indicates where the measured tilt would equal the predicted tilt.

technique like STEM because it can cover orders of magnitude larger lateral space by measuring only a few scan lines per structure instead of having to FIB a thin slice out of each of the structures.

We note that lattice tilting is also observed across grain boundaries in oxide systems, for example across a twin grain boundary in tetragonal $\mathrm{SrTiO}_{3}[162]$ or $\mathrm{PbTiO}_{3}[163]$. In both cases the tilting can be described using a simplified version of equation (4.1), since across the domain wall $a$ and $c$ are exchanged and the 'ramp' angle can be defined by $\arctan (a / c)$, leading to $\gamma=2 \arctan (c / a)-90^{\circ}$. A tilted lattice also appears across a grain boundary between rhombohedrally $(R)$ distorted and tetragonally $(T)$ distorted $\mathrm{BiFeO}_{3}$ for specific substrate strain [164-166]. Here equation (4.1) can be used directly by using the pseudocubic $c / a$ ratios associated with the $R$ and the $T$-phase and taking the inclination of the grain boundary as the ramp angle. Figure 4.9 shows the measured lattice tilt versus the predicted lattice tilt using equation (4.1) for the three materials described above and the LSCO/NCCO system. The measured tilt and the model tilt are determined using the main text and the TEM figures in references [162-164]. The two data points for $\mathrm{BiFeO}_{3}$ correspond to the $T / R$ and the $R / T$ configuration. The dotted line indicates where the measured tilt would equal the predicted tilt. From this we see that the lattice tilt in all four systems can be well described with the facet matching model for strain accommodation. It also means that the LSCO/NCCO ramp-edge junctions have a crystalline grain boundary contact at the interface. Firstly, this is important for the measurements discussed in chapter 3 , but secondly, it also means that a ramp-edge structure can potentially be used to tailor specific, artificial grain boundaries in piezoelectric materials like $\mathrm{BiFeO}_{3}$ through strain engineering. For a periodic mesa structure with ramps on both sides in e.g. $\mathrm{SrTiO}_{3}$, the $\mathrm{BiFeO}_{3}$ can be forced to nucleate in the $T$-phase on the 


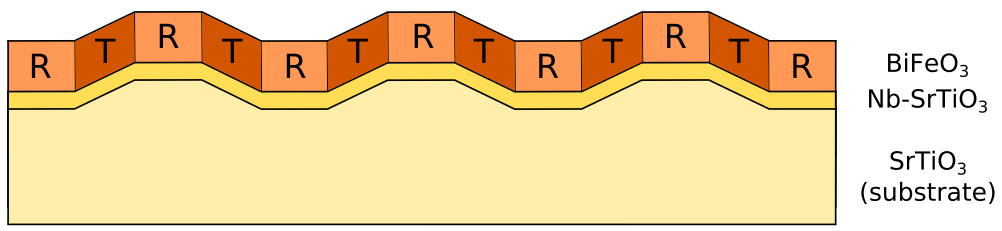

Figure 4.10: Schematic cross-section of the suggested geometry to achieve artificial multiphase $\mathrm{BiFeO}_{3}$ by energy minimization driven domain formation. The ramp angle is chosen such that the $T$-phase of $\mathrm{BiFeO}_{3}$ prefers to nucleate on the ramp, whereas the $R$-phase will form on the other parts of the structure. The Nb-SrTiO 3 layer is added as a bottom electrode and will act as a restoring layer for damage on the ramp due to the argon ion milling.

ramps by choosing a specific ramp angle (around $26^{\circ}$ ), whereas the $\mathrm{BiFeO}_{3}$ will nucleate in the $R$-phase on the other parts of the structure, see figure 4.10 . This would be an artificial realization of the mixed phase state of $\mathrm{BiFeO}_{3}$ as observed by Zeches et al. [164].

\subsection{Conclusions}

We have shown that nano-focused scanning X-ray diffraction can be a powerful tool for non-destructive device characterization. This is particularly useful as an intermediate characterization, it can be done without interfering with other measurements or requiring new sample fabrication. New insights can be applied in further measurements on the same device. Nano-XRD is able to act as a local probe for buried layers and can identify areas of interest for further investigation using higher resolution techniques like HAADF-STEM. For nano-scale devices the beam size of $\sim 300 \mathrm{~nm}$ offers limited resolution, but, as we have shown, sufficient to detect features the size of the beam. Nano-XRD has allowed us to identify a tilted phase of LSCO in LSCO/NCCO ramp-edge junctions that we have confirmed by HAADF-STEM. Furthermore, we argue that the origin of the tilting in a interplay between lattice strain and the ramp angle, promoting the LSCO to nucleate in a tilted phase on the exposed NCCO facets at the ramp interface as a method for strain accommodation. With this, we predict that this will occur for material combinations that have a sufficiently large in-plane lattice mismatch and have a different $c / a$ ratio. Notable example are the combination YBCO/LSCO and LSCO/STO or LSCO/LSAT, where a similar tilt effect is expected to what we have observed in the LSCO/NCCO system. Finally, we have shown that lattice tilting also appears in domain walls in various piezoelectric materials, following the same general equation. Therefore, we conclude that (1) the LSCO/NCCO rampedge contact is a crystalline contact similar to a domain wall or grain boundary and (2) ramp-edge structures can potentially be used to create artificial domain structures in piezoelectric materials by patterned substrates. 


\section{Appendices}

\section{A Supplementary figures}

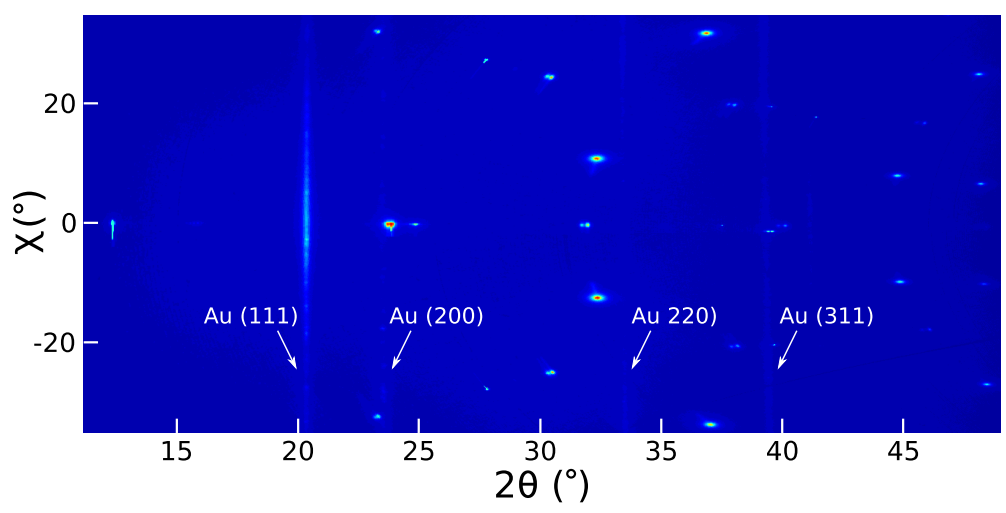

Figure A.1: Full $\theta-2 \theta$ scan on the junction area (3) in figure $4.2(\mathrm{c})$. The image is projection corrected to show a map of $\chi$ versus $2 \theta$, the signal of the polycrystalline gold particles appears as vertical lines that have been labelled by their respective miller indices. Substrate peaks appear weak as frames with oversaturation have been removed in the frame summation procedure.
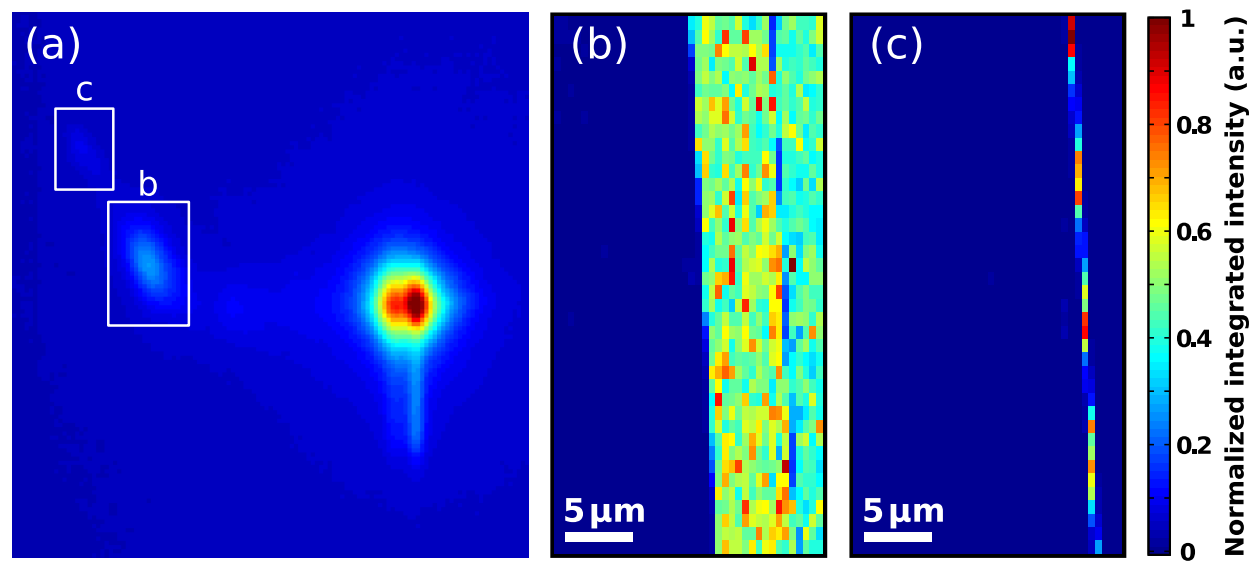

Figure A.2: Integrated nXRD intensity grid scans for LSCO (107). (a) Closeup of the NCO/NCCO/LSCO (107) peaks for a sum of all nXRD frames on a logarithmic color scale. Boxes $b$ and $c$ are used for the intensity mapping of (b) and (c). (b) Intensity map of the $c$-axis aligned LSCO phase. (c) Intensity map of the $3^{\circ}$ tilted LSCO phase. No intensity is observed outside the ramp area. A background is subtracted from both maps and the signal is normalized to the highest intensity pixel. The color scale refers to both (b) and (c). 


\section{B Lattice tilt}

For the derivation of equation (4.1) we assume that the tilting originates from a matching of the same $(h 0 l)$ planes of the two materials. We will use the schematic diagram in figure 4.7 (a) for reference. The $(h 0 l)$ facet of 'lattice A' will make an angle $\alpha$ with the $(a, b)$-plane, which is also the ramp angle. The same facet in 'lattice B' will make a different angle $\alpha^{\prime}$, in this case larger than $\alpha$. Both angles can be expressed using the lattice parameters of the two lattices,

$$
\tan \alpha\left(\alpha^{\prime}\right)=\frac{n c_{A(B)}}{m a_{A(B)}}
$$

where $n$ and $m$ are the number of unit cells that define the angle associated with the $(h 0 l)$ plane and $a$ and $c$ are the in and out-of-plane lattice parameters. The tilt angle can now be defined as $\gamma=\alpha^{\prime}-\alpha$, where $\alpha^{\prime}$ can be eliminated using

$$
\frac{n}{m}=\frac{a_{A}}{c_{A}} \tan \alpha,
$$

to get

$$
\tan \alpha^{\prime}=\frac{a_{A} c_{B}}{c_{A} a_{B}} \tan \alpha
$$

which gives for $\gamma$,

$$
\gamma=\arctan \left(\frac{a_{A} c_{B}}{c_{A} a_{B}} \tan \alpha\right)-\alpha .
$$

These equations can also be used to get a figure for the lattice mismatch between the tilted planes. We associate a length $L\left(L^{\prime}\right)$ with the $(h 0 l)$ facet of lattice $\mathrm{A}(\mathrm{B})$,

$$
L\left(L^{\prime}\right)=\frac{m a_{A(B)}}{\cos \alpha\left(\alpha^{\prime}\right)}
$$

This we use to express a lattice mismatch

$$
\frac{L-L^{\prime}}{L}=1-\frac{a_{B} \cos \alpha}{a_{A} \cos \alpha^{\prime}},
$$

where equation (4.4) can be used again to express $\cos \alpha^{\prime}$ in terms of $\alpha$ and the lattice parameters of the two materials. 



\section{CHAPTER 5}

\section{NCCO-LSCO bilayers and c-axis contacts}

This chapter presents an overview of the fabrication and properties of $\mathrm{Nd}_{1.85} \mathrm{Ce}_{0.15} \mathrm{CuO}_{4}-\mathrm{La}_{1.85} \mathrm{Sr}_{0.15} \mathrm{CuO}_{4}$ (NCCO-LSCO) bilayers on LSAT $\left(\left[\mathrm{LaAlO}_{3}\right]_{0.3}\left[\mathrm{Sr}_{2} \mathrm{AlTaO}_{6}\right]_{0.7}\right)$ substrates. A deposition temperature and pressure profile is devised with the specific annealing requirements of the materials in mind to ensure superconductivity in both layers for the LSAT-NCCOLSCO and the LSAT-LSCO-NCCO configuration. The first configuration is investigated further by high-angle annular dark-field transmission electron microscopy (HAADF-STEM) and the LSCO layer is characterized using Xray absorption spectroscopy (XAS). HAADF-STEM shows that the interface between NCCO and LSCO is not sharp due to the intrinsic roughness of the NCCO layer. In $c$-axis transport, non-linear $I V$ characteristics are measured, indicative of a barrier at the interface. XAS reveals the presence of $\mathrm{Cu}^{1+}$ in the LSCO layer and shows the disappearance of the signature for the Zhang-Rice singlet state. The appearance of $\mathrm{Cu}^{1+}$ is tentatively attributed to oxygen vacancies.

\subsection{Introduction}

A $c$-axis contact is one of the cleanest ways to make a junction between two oxides, since it can be fabricated completely without breaking vacuum and requires no intermediate steps like dry or wet etching. In this chapter we will discuss the $c$-axis contact between NCCO and LSCO. This contact is expected to differ from the in-plane ramp-edge contacts described in the previous chapters due to the inherent anisotropy of the cuprates. As we have seen in chapter 1, the ThomasFermi screening length is expected to be longer in the $c$-axis direction. This allows any charge transfer and depletion effects to occur over a longer distance, potentially spanning a number of $\mathrm{CuO}_{2}$ planes. For the in-plane contacts we have seen that the band alignment is dominated by interface hybridization, an effect that is expected to be less strong in the $c$-axis direction due to the quasi-2D nature of the cuprates. This allows for unconventional Mott depletion as predicted for oppositely doped cuprates stacked in the $c$-axis direction [12]. A $c$-axis contact with the oppositely doped $\mathrm{CuO}_{2}$ planes parallel is also the ideal configuration to look for excitonic effects in strongly correlated systems [13-16]. 
In the following we will consider two configurations, LSAT-NCCO-LSCO, denoted by 'LNL', and LSAT-LSCO-NCCO, denoted by 'LLN', NCCO refers to $\mathrm{Nd}_{1.85} \mathrm{Ce}_{0.15} \mathrm{CuO}_{4}$ and $\mathrm{LSCO}$ to $\mathrm{La}_{1.85} \mathrm{Sr}_{0.15} \mathrm{CuO}_{4}$, unless otherwise specified, see figure 5.1(a). LNL is the standard configuration, where the first layer that is deposited (NCCO), is also deposited at the highest deposition temperature. The LLN configuration is the inverted configuration, where the last layer (NCCO) is deposited at the highest temperature. Together these configurations form the building blocks for multilayer devices, of particular interest in e.g. X-ray absorption spectroscopy (XAS) experiments to study interface effects and device fabrication for thermoelectrics [16].

In this chapter we will discuss the fabrication of LLN and LNL devices and the characterization of these devices by X-ray diffraction (XRD), atomic force microscopy (AFM) and high-angle annular dark-field scanning transmission electron microscopy (HAADF-STEM). In-plane transport measurements are performed on the LNL and LLN configuration and the LNL configuration is also measured in a $c$-axis configuration. Finally, we use XAS to probe the electronic state of the LSCO top layer in the LNL configuration.

\subsection{Deposition conditions}

For the successful in situ combination of NCCO and LSCO, we use the knowledge from chapter 2 where we have shown that $\mathrm{NCCO}$ grown from a target with extra copper added is robust under oxygen annealing and that vacuum annealing prior to the oxygen annealing is essential for superconductivity. We expand on this by exploring the properties of the $\mathrm{NCCO}$ when it is capped with a $\mathrm{SrTiO}_{3}$ (STO) to develop a deposition temperature-pressure profile suitable for optimal combination of NCCO with a $p$-type material like LSCO. STO is used instead of LSCO to get an insulating capping layer, so only the NCCO layer will be measured. We have investigated three procedures; in the first (I), the STO cap is deposited before vacuum annealing the sample at $740^{\circ} \mathrm{C}$, after that the sample is cooled down in vacuum and oxygen is introduced at $600^{\circ} \mathrm{C}$. The annealing procedure is the same as the oxygen annealing procedure as described in chapter 2, table 2.1. The second procedure (II) is the same as the first, but here an extra $8 \mathrm{~min}$ vacuum annealing is performed before the STO cap is deposited. In the final procedure (III) the NCCO is vacuum annealed at $740^{\circ} \mathrm{C}$ for $8 \mathrm{~min}$ and then allowed to cool down to $600^{\circ} \mathrm{C}$ before heating the sample again to $700^{\circ} \mathrm{C}$ for the STO deposition. The deposition pressure of STO of $0.1 \mathrm{mbar}$ oxygen is set at $680^{\circ} \mathrm{C}$ in order to do pre-ablation and to limit the residence time at $700{ }^{\circ} \mathrm{C}$.

Figure 5.1(b) shows transport measurements on samples grown using the three procedures described above, labeled I-III in the figure. The STO capping can be seen to be a very effective oxygen barrier. No superconductivity is observed for the first procedure where there is only vacuum annealing after deposition of the capping layer; the sample only shows an upturn in the resistance with decreasing temperature. The second sample (II), which was vacuum annealed 
(a)

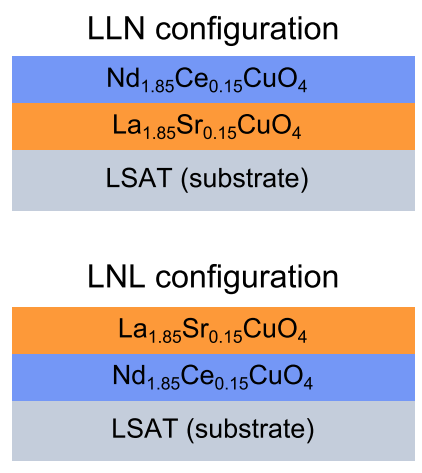

(b)

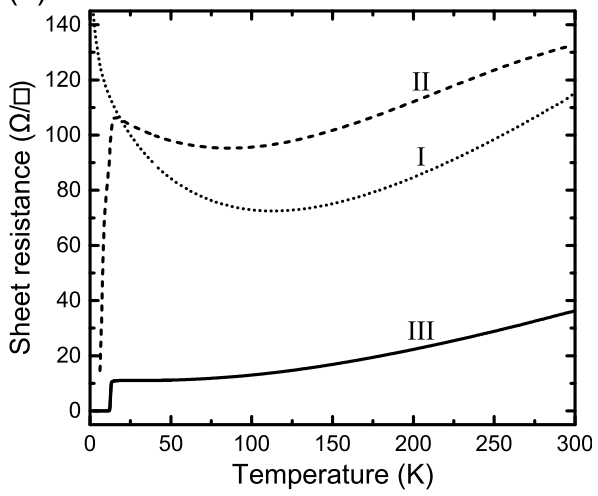

Figure 5.1: (a) Schematic representation of the LLN and the LNL configurations. Both the NCCO and the LSCO layers are $150 \mathrm{~nm}$ thick. (b) Sheet resistance for NCCO with STO capping layer grown using three different procedures: (I) vacuum annealing after STO capping, (II) vacuum annealing before and after STO capping, and (III) vacuum annealing and dip to $600{ }^{\circ} \mathrm{C}$ before STO capping. All procedures are followed by a standard oxygen annealing.

before deposition of the STO cap, already shows a superconducting transition, but fails to become fully superconducting. It is interesting to note that procedure I gives a homogeneous, non-superconducting film, whereas procedure II gives a partly superconducting, but highly inhomogeneous result with a larger net resistance than what is obtained using procedure I. The sample grown using the last procedure shows a sharp superconducting transition and a sheet resistance of the same order of magnitude as the samples described in chapter 2. This experiment indicates that not only the vacuum dwell of $8 \mathrm{~min}$ at $740^{\circ} \mathrm{C}$ is crucial for superconductivity, but also (part) of the subsequent cool down procedure. This resonates with a recent extensive annealing study by Krockenberger et al. on $\mathrm{Pr}_{2-\mathrm{x}} \mathrm{Ce}_{\mathrm{x}} \mathrm{CuO}_{4}$ [41]. They have developed a two step annealing procedure where in an initial high temperature low pressure annealing oxygen is removed from the $\mathrm{CuO}_{2}$ planes. At a subsequent vacuum annealing at a lower temperature, the $\mathrm{CuO}_{2}$ lattice is restored by incorporating apical oxygen that was not removed in the initial step. This clearly indicates that the cool down procedure is also of importance to the superconducting properties of the $n$-type cuprates.

From the results with the STO capping layer, we have derived a temperaturepressure profile for the combination of $\mathrm{NCCO}$ with LSCO, as depicted in figure 5.2 (a). After the deposition of the $\mathrm{NCCO}$ bottom layer at $820^{\circ} \mathrm{C}$, the sample is cooled down in deposition pressure to $740^{\circ} \mathrm{C}$. Here, the sample is vacuum annealed for $8 \mathrm{~min}$ after which it is cooled down to $600^{\circ} \mathrm{C}$ at $10^{\circ} \mathrm{C} \mathrm{min}-1$. Next, the sample is warmed up again to $700^{\circ} \mathrm{C}$ in vacuum and the deposition pressure for LSCO of $0.13 \mathrm{mbar}$ is set at $680^{\circ} \mathrm{C}$. After the LSCO deposition the sample is cooled down and at $680^{\circ} \mathrm{C}$ the PLD chamber is flooded to 1 bar oxygen. The annealing 
(a)

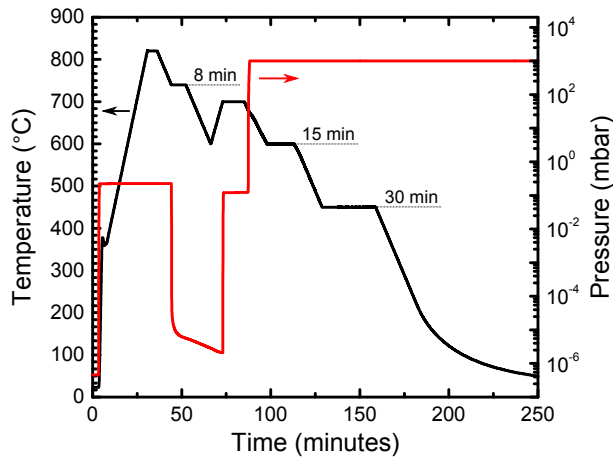

(b)

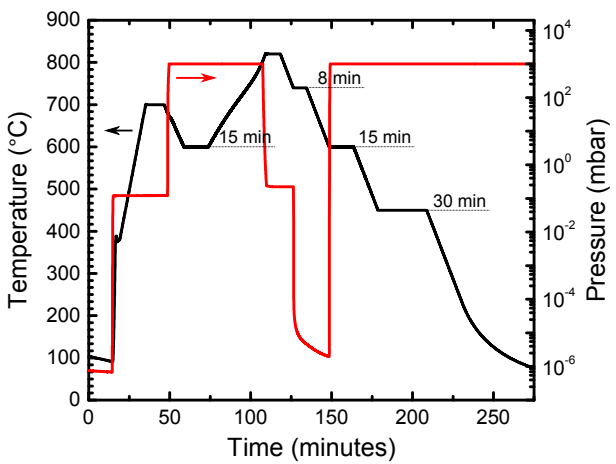

Figure 5.2: Temperature (black curve, left scale) and (oxygen) pressure (red curve, right scale) profile used in the fabrication of the LNL configuration (a) and the LLN configuration (b). Annealing times are indicated.

procedure is finished with a dwell of $15 \mathrm{~min}$ and $600{ }^{\circ} \mathrm{C}$ and a dwell of $30 \mathrm{~min}$ at $450{ }^{\circ} \mathrm{C}$ to ensure oxygenation of the LSCO layer.

A similar procedure is devised for the reverse order of the layers, see figure 5.2(b). The advantage of reversing the order is that LSCO films tend to have a lower roughness than NCCO films, where the growth is more island-like. This creates a better-defined interface between the two materials. The downside is that in order to this, the LSCO layer needs to be either deposited at a higher temperature than usual, or has to be heated up above the deposition temperature of $700{ }^{\circ} \mathrm{C}$ to reach the deposition temperature of $820^{\circ} \mathrm{C}$ of $\mathrm{NCCO}$. Deposition temperatures for LSCO between 700 and $780^{\circ} \mathrm{C}$ have been reported in the literature [167-169]. We have explored deposition temperatures up to $780^{\circ} \mathrm{C}$ and find that the film conductivity becomes inhomogeneous at the highest temperatures. The best result is obtained at the original deposition temperature of $700{ }^{\circ} \mathrm{C}$ and therefore we have chosen to heat up the sample after deposition to reach the deposition temperature of NCCO. We employ a similar annealing scheme as before, see figure 5.2(b): after deposition of the LSCO layer we perform part of the standard annealing procedure by cooling down to $600{ }^{\circ} \mathrm{C}$ in 1 bar oxygen and leaving the sample there for $15 \mathrm{~min}$. Then, the sample is carefully warmed up again to $820^{\circ} \mathrm{C}$ (still in 1 bar oxygen), ensuring that the heater voltage and current do not become too high, to prevent damage to the resistance coil of the PLD heater. At $800{ }^{\circ} \mathrm{C}$ the pressure is reduced to the deposition pressure for NCCO to protect the PLD heater and to do pre-ablation. After the deposition of NCCO we perform the standard anneal for NCCO down to $600{ }^{\circ} \mathrm{C}$ where we switch to the procedure for LSCO, as was also detailed in chapter 2 . 


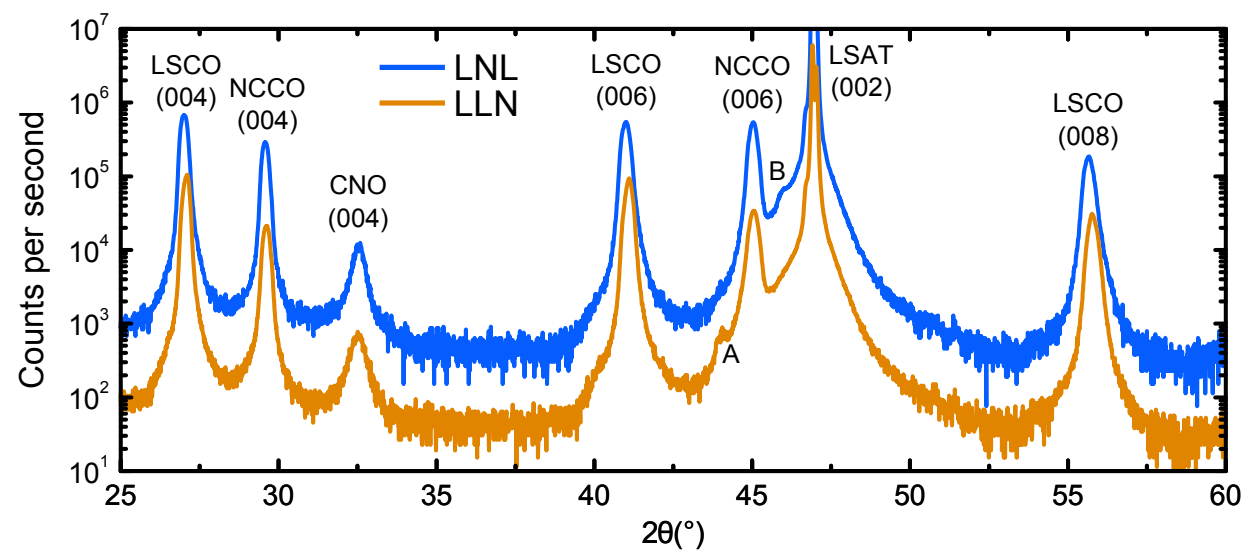

Figure 5.3: XRD $\theta-2 \theta$ scans for samples in the LNL (blue) and LLN (orange) configuration, normalized to LSAT (002) and offset for clarity. LSCO, NCCO, $(\mathrm{Nd}, \mathrm{Ce})_{2} \mathrm{O}_{3}$ and LSAT peaks are labeled. Two additional peaks are labeled $\mathrm{A}$ and $\mathrm{B}$, their origin is not known.

\subsection{Sample characterization}

Samples of the LNL and the LLN configuration were fabricated by pulsed laser deposition with each of the layers $150 \mathrm{~nm}$ thick. Both NCCO and LSCO are deposited at a fluency of $1.2 \mathrm{~J} \mathrm{~cm}^{-2}$, a spot size of $5.7 \mathrm{~mm}^{2}$ and a repetition rate of $4 \mathrm{~Hz}$. The NCCO is deposited at $820^{\circ} \mathrm{C}$ and 0.25 mbar oxygen pressure and the LSCO is deposited at $700^{\circ} \mathrm{C}$ and 0.13 mbar oxygen pressure. The samples are characterized structurally by XRD, AFM and HAADF-STEM combined with energy-dispersive X-ray (EDX) spectroscopy.

\subsubsection{X-ray diffraction}

Figure 5.3 show XRD $\theta-2 \theta$ spectra comparing the two configuration, the spectra are normalized to LSAT $\left(\begin{array}{lll}0 & 2\end{array}\right)$ and displaced for clarity. Reflections for NCCO, LSCO and $(\mathrm{Nd}, \mathrm{Ce})_{2} \mathrm{O}_{3}$ are indicated. Comparing LLN to LNL, the LSCO peak intensities is higher for the LLN configuration, the opposite is true for the NCCO peak intensities, where the highest intensity is found for the LNL configuration. Taking the full width at half maximum (FWHM) of the (0 04$)$ peaks as an indication of the film quality ${ }^{\dagger}$, we find a FWHM of $0.25^{\circ}\left(0.27^{\circ}\right)$ for NCCO (LSCO) in the LNL configuration and $0.26^{\circ}\left(0.23^{\circ}\right)$ for NCCO (LSCO) in the LLN configuration, see also table 5.1. Moving the NCCO layer from the bottom to the top layer results

$\dagger$ The finite thickness broadening contribution to the FWHM following the Scherrer equation, see chapter 2 , is around $0.06^{\circ}$ for a $150 \mathrm{~nm}$ film. For low angles, the $\mathrm{Cu} K \alpha_{1,2}$ splitting is still small and therefore the FWHM of the (004) peaks can be taken as an indication of crystal quality. 
in an increase of the FWHM by only $4 \%$, within the measurement uncertainty we can say that the FWHM does not change for NCCO. For LSCO we find an increase of $15 \%$ when grown on NCCO, compared to it being grown directly on LSAT. We attribute this increase to the extra lattice mismatch between NCCO and LSCO as compared to LSAT and the intrinsic roughness of the NCCO surface.

Two additional peaks can be seen in the spectra for the LNL and the LLN configuration labeled A and B. Peak A appears in the LLN sample at $44.05^{\circ}$ and peak B appears in the LNL sample at $45.9^{\circ}$. Both peaks also appear in both NCCO and LSCO samples grown on either LSAT or STO. It is difficult to determine the origin of a peak from a single reflection, but the peaks are not related to a different orientation of either NCCO, LSCO or LSAT. They are also not related to either the gold of the electrode or the silver from the silver paint used to mount the samples for PLD. Obvious parasitic phase candidates like (Nd,Ce) $)_{2} \mathrm{O}_{3}, \mathrm{SrO}$, $\mathrm{La}_{2} \mathrm{O}_{3}$ or any $\mathrm{Cu}_{\mathrm{x}} \mathrm{O}_{\mathrm{y}}$ phase also do not have reflections at these positions. The peaks do not always appear and the peak at $45.9^{\circ}$ was measured in an $\mathrm{NCCO}$ film and then disappeared after a measurement in the PPMS. This suggests that the peak is either related to a surface phase or even something not related to the sample, such as the sample holder or the adhesive tape used. Since the peaks also appear in the single films of $\mathrm{NCCO}$ and $\mathrm{LSCO}$ and since they have a low intensity, even compared to the $(\mathrm{Nd}, \mathrm{Ce})_{2} \mathrm{O}_{3}$ parasitic phase, we will ignore them in our analysis.

Using a wider spectrum collected from 10 to 115 degrees, we can determine the $c$-axis length for LSAT, NCCO and LSCO using the angular peak positions and Bragg's law $(n \lambda=2 d \sin \theta)$. For LSAT we use the wavelength $\lambda$ associated with $\mathrm{Cu} K \alpha_{1}(1.54056 \AA)$, since the substrate peaks are sharp enough to resolve the $\mathrm{Cu} K \alpha_{1,2}$ splitting; for the film peaks this is not the case and we use the general wavelength for $\mathrm{Cu} K \alpha\left(1.5418 \AA\right.$, assuming the $K \alpha_{2} / K \alpha_{1}$ ratio is 0.5$)$. In the procedure we allow for a constant shift of the entire spectrum to account for instrumental misalignment and potential tilting of the layers. The peak positions are determined by fitting a Pseudo-Voigt profile ${ }^{\dagger}$ with a constant background to each peak. Using the constant shift as free parameter, we minimize the sum of the squared differences between the $d$-values for each peak and the average of the $d$-values. The results are summarized in table 5.1. The error quoted in the table is a $2 \sigma$ statistical spread of the set of $c$-axis values for each peak that corresponds to the obtained shift.

From the values for $\mathrm{NCCO}$ we can conclude that the residual strain in the NCCO layers is comparable for both configurations, the bulk value of $12.08 \AA$ [38] falls

${ }^{\dagger}$ A Voigt profile is a convolution of a Gaussian line shape and a Lorentzian line shape. A PseudoVoigt profile is an approximation of the Voigt profile and is a linear combination of a Gaussian and a Lorentzian:

$$
P S V(x)=C_{0}+C_{1}\left(f \frac{2}{\pi} \frac{W}{4\left(x-x_{0}\right)^{2}+W^{2}}+(1-f) \frac{\sqrt{4 \ln 2}}{\sqrt{\pi} W} \exp \left[-\frac{4 \ln 2}{W^{2}}\left(x-x_{0}\right)^{2}\right]\right),
$$

where $C_{0,1}$ are constants, $f$ is a fraction between 0 and 1 that determines the relative Gaussian vs. Lorentzian contribution, $W$ is the full width at half maximum and $x_{0}$ is the position of the maximum, that is used in the analysis. 
Table 5.1: $c$-axis values, with statistical error in brackets, peak shifts for NCCO, LSCO and LSAT and the full width at half maximum (FWHM) of the (0 04 ) peak of NCCO and LSCO and the (002) peak of LSAT as determined by XRD for the LNL and the LLN configuration.

\begin{tabular}{lcccccc}
\hline & \multicolumn{3}{c}{ LNL } & & \multicolumn{3}{c}{ LLN } & \\
& $c$-axis $(\AA)$ & shift $\left(^{\circ}\right)$ & FWHM $\left(^{\circ}\right)$ & $c$-axis $(\AA)$ & shift $\left(^{\circ}\right)$ & FWHM $\left(^{\circ}\right)$ \\
\hline LSAT & $3.872(1)$ & -0.024 & 0.07 & $3.872(1)$ & -0.025 & 0.07 \\
NCCO & $12.075(5)$ & 0.023 & 0.25 & $12.074(7)$ & -0.015 & 0.26 \\
LSCO & $13.213(2)$ & -0.013 & 0.27 & $13.193(3)$ & -0.040 & 0.23 \\
\hline
\end{tabular}

within the error bar of the measurement for both samples. NCCO should be compressively strained on LSAT, if strained at all, resulting in an elongation of the $c$-axis, assuming a constant unit cell volume. In chapter 2 we have reported the same value of $12.08 \AA$ for single $\mathrm{Nd}_{1.85} \mathrm{Ce}_{0.15} \mathrm{CuO}_{4}$ films grown under similar conditions.

For both configurations, the LSCO $c$-axis length is shorter compared to the bulk value of $13.227 \AA$ for $\mathrm{La}_{2-\mathrm{x}} \mathrm{Sr}_{\mathrm{x}} \mathrm{CuO}_{4} x=0.15$ [71], as is expected from tensile strain on both LSAT and NCCO. The in-plane lattice mismatch is $2.4 \%$ and $4.4 \%$, respectively $[38,71]$. From the EDX measurements in chapter 3 we have an estimated doping level of $x=0.17$; around optimal doping the expected $c$-axis length becomes larger for higher doping levels, by about $0.01 \AA$ for $x=0.17$ [140]. The $c$-axis length for LSCO in the LLN configuration is comparable to what we find for a single LSCO film on LSAT: 13.184(6) $\AA$. The layer on LSAT shows the shortest $c$-axis length, while the lattice mismatch is biggest on NCCO. This suggests that the LSCO layer in the LNL configuration is more relaxed. The larger lattice mismatch may allow for an easier formation of defects and dislocations at the interface, which is reflected in the larger FWHM for the $(004)$ peak as compared to the LLN configuration. Ce diffusion into the LSCO layer would result in a decrease of the $c$-axis length due to the smaller ionic radius of Ce [70], this effect works in the same direction as the strain and can therefore not be confirmed nor excluded from this measurement.

The intensity of the peak corresponding to the $(004)$ reflection of the parasitic $(\mathrm{Nd}, \mathrm{Ce})_{2} \mathrm{O}_{3}$ phase in the NCCO layer does not vary significantly and is of the order of a few percent of the peak intensity of NCCO (004). Scatter in the intensity of this peak from deposition to deposition, possibly due to target inhomogeneity, is too large to observe a significant difference. From the very small change in the FWHM of the NCCO (ll 04 ) peak, it is not expected to differ much, in chapter 2 it was shown that the appearance of the parasitic phase was accompanied by an increase in FWHM of the NCCO (0 044$)$ peak. One difference is the potential formation of a thin layer of $(\mathrm{Nd}, \mathrm{Ce}){ }_{2} \mathrm{O}_{3}$ at the interface between LSCO and NCCO in the LLN configuration due to the lattice mismatch between LSCO and NCCO. We have observed something similar for the LSAT-NCCO interface in the TEM 

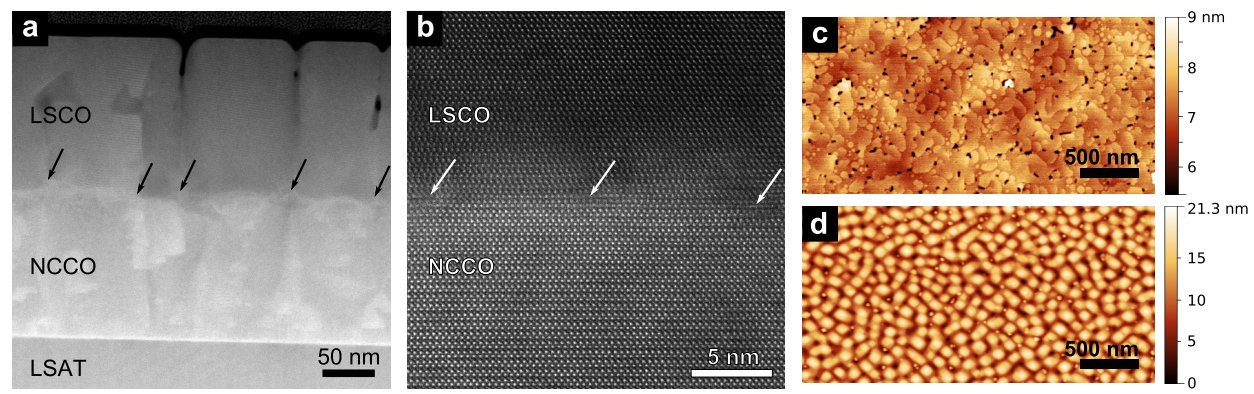

Figure 5.4: (a) Low magnification HAADF-STEM cross-section of a NCCOLSCO bilayer. The two layers and the substrate are indicated. The black arrows indicate defects that nucleate voids seen in the LSCO layer. (b) High magnification close-up of the NCCO-LSCO interface. An overlap layer with disorder $\sim 50 \mathrm{~nm}$ wide is observed at the interface, with no additional phases. Small misorientation in the LSCO can be observed as distortion of the atomic lattice. (c) AFM image of a NCCO-LSCO bilayer (LNL). (d) AFM image of a LSCO-NCCO bilayer (LLN).

study described in chapter 2, where we have found occasional thin sections of the $(\mathrm{Nd}, \mathrm{Ce})_{2} \mathrm{O}_{3}$ phase right at the interface between LSAT and $\mathrm{NCCO}$, even in the case of NCCO films grown from targets with extra copper added. This is also seen for the interface between LSCO and another n-type cuprate $\mathrm{Pr}_{2-\mathrm{x}} \mathrm{Ce}_{\mathrm{x}} \mathrm{CuO}_{4}$ [62]. This potential interface layer might not be thick enough to show up in the XRD spectrum. If so, the interface layer must be of the order of a nanometer, as the $(\mathrm{Nd}, \mathrm{Ce})_{2} \mathrm{O}_{3}$ inclusions normally found in the NCCO films are several nanometers in size and do show up in XRD measurements. In the next section and in chapter 3 we show that such an interlayer does not form when LSCO is grown on NCCO, i.e. in the LNL configuration. The LLN configuration remains to be investigated.

\subsubsection{HAADF-STEM and AFM}

Figure 5.4 shows the results for the HAADF-STEM and the AFM measurements. HAADF-STEM is only performed on an LNL sample. The sample is prepared for TEM by focused ion beam (FIB) etching, as was described previously in $\S 4.2 .2$. Figure 5.4(a) shows a low magnification cross-section of the NCCOLSCO interface, both layers and the substrate are indicated. The interface can be identified and it is not smooth, due to the intrinsic roughness of the NCCO layer. The LSCO consists of distinct crystal blocks with deep voids between them, extending 50-100 nm. These same voids are also measured by AFM, figure 5.4(c), where they appear as black spots with a density of around 60 per $\mu^{2}$. The voids are also found for LSCO films grown directly on LSAT, but at a lower density. They may be a consequence of strain between LSAT and LSCO or NCCO and LSCO. Both LSAT and NCCO provide a template for tensile strain in the LSCO. 
In $\S 5.3 .1$ we have concluded that LSCO grown on NCCO is relaxed more than LSCO grown on LSAT, although the lattice mismatch is bigger. The most likely scenario is that strain is accommodated through defects that form into the voids we observe using HAADF-STEM and AFM. The black arrows in figure 5.4(a) indicate where the voids nucleate at the NCCO-LSCO interface. Most of the defects can be seen to propagate through the NCCO layer. Thus, defects in the NCCO layer allow the LSCO top layer to relax through the formation of voids.

Figure 5.4(b) shows a high resolution close-up of the NCCO-LSCO interface. A $\sim 5 \mathrm{~nm}$ transition can been seen between the NCCO and the LSCO, where the LSCO lattice is distorted due to overlapping phases. The white arrows indicate sites of defects or dislocations. The LSCO can been seen to have a small internal misorientation, as evidenced by the distorted lattice toward the right edge of the figure. No additional phases are observed at the interface other than NCCO and LSCO.

EDX spectroscopy measurements, performed together with the HAADF-STEM measurements, show no interdiffusion of elements across the interface, similar to what was also observed in chapter 3 .

As can be seen from the low magnification TEM, the surface of the LSCO grains is very smooth. This is also confirmed by AFM, figure 5.4(c). The film shows an RMS roughness of $0.5 \mathrm{~nm}$, including $2-3 \mathrm{~nm}$ particles ( $100 \mathrm{~nm}$ lateral size, $1-2$ particles per $\mu^{2}$ ) and the voids discussed before. Compared to LSCO grown on LSAT, the bilayer LSCO has a higher density of voids, but a lower density of particles. The nature of the particles is unknown, but this suggests they may also be related to the strain relaxation.

The surface of the LLN configuration is dominated by the three dimensional island growth of NCCO, see figure $5.4(\mathrm{~d})$. The grain size is $50-100 \mathrm{~nm}$, the surface has a RMS roughness of $3.6 \mathrm{~nm}$ with a peak-to-peak value of around $10 \mathrm{~nm}$. Here, also voids are observed between the grains, that we expect to also originate from strain accommodation. The same structure, including the voids and the small particles between the grains is also seen for NCCO grown directly on LSAT. This also follows from the XRD data, where we see that in both cases the NCCO is mostly relaxed. LSCO films grown on LSAT provide a smooth enough template to not influence the surface structure of the NCCO in a major way.

\subsection{Results and discussion}

\subsubsection{In-plane transport}

In-plane transport measurements are performed by depositing gold contacts in the corners of the bilayer films and measuring the entire stack in a common van der Pauw configuration. For the LLN configuration, the contacts are deposited directly on top; for the LNL configuration, the LSCO layer is removed by argon 
ion milling in a square ring around the edges of the sample and the contacts are deposited on the NCCO layer. This last procedure is performed to ensure that the superconducting transition of both layers can be observed. Without etching, the bottom layer would be shunted by the top layer as soon as the LSCO becomes superconducting. The top two panels of figure 5.5(a) show the combined sheet resistance versus temperature for the two bilayers and the inset shows the measurement configuration. Reference measurements on a $70 \mathrm{~nm}$ NCCO film and a $150 \mathrm{~nm}$ LSCO film are shown in the bottom two panels. In the bilayer both layers are $150 \mathrm{~nm}$ thick. The bilayers show superconducting transitions for both NCCO and LSCO, close to $T_{c}$ of the individual films, with NCCO showing the lower $T_{c}$.

The LLN configuration shows a superconducting transition for LSCO, at a temperature lower than the reference film, due to the extra heating step required for the growth of the NCCO layer. The superconducting transition for the NCCO cannot be clearly observed, but a feature is observed around the critical temperature of the reference film. The gray data points show a close-up of this feature, 50 times magnified. The superconducting nature of the NCCO layer is verified by magnetotransport, as will be discussed later.

The LNL configuration shows a superconducting transition for both LSCO and NCCO. For NCCO we observe a small decrease in $T_{c}$, in accordance with the observations in chapter 2 . The transition appears to be more inhomogeneous; this is most likely caused by the argon ion milling and the measurement deviating from an ideal van der Pauw measurement. The LSCO in the LNL configuration shows a $T_{c}$ that is about $1 \mathrm{~K}$ higher than the reference film. We attribute this to the relaxation of the LSCO lattice when grown on $\mathrm{NCCO}$ as discussed in the previous section (§5.3.1). Tensile strain, which is more present in the reference sample, is known to suppress the $T_{c}$ of LSCO [170].

Figure 5.5(b) shows the temperature dependence of the resistivity for the LLN configuration for a larger temperature range. The dashed gray curve shows the equivalent resistivity for the reference films of figure 5.5(a) in parallel, scaled to a thickness of $150 \mathrm{~nm}$ for both. The bilayer shows a higher resistance than what would be expected from the individual layers. Looking at the resistivity of the reference layers this can mean that LSCO is dominating the resistance or that the resistivity of either of the two layers is higher than that of the reference samples. From the bilayer geometry we expect the NCCO to dominate, since it is the top layer. This leaves a higher resistivity for either of the two layers with respect to the reference samples as the most likely scenario. The solid gray curve is a least squares fit where a scaling factor is added to the resistivity of each layer in the parallel circuit. Assuming no variation in layer thickness, this gives an additional relative conductivity contribution of 2.27:1 for LSCO:NCCO, indicating that LSCO contributes more than would be expected from a simple parallel circuit. In absolute terms the fit gives a $10 \%$ reduction of the effective resistance of the LSCO layer and a resistance that is two times higher for the NCCO layer. This increase in resistance for the NCCO layer is in accordance with what we have observed in chapter 2, where $\mathrm{NCCO}$ films that were annealed in oxygen after 
(a)
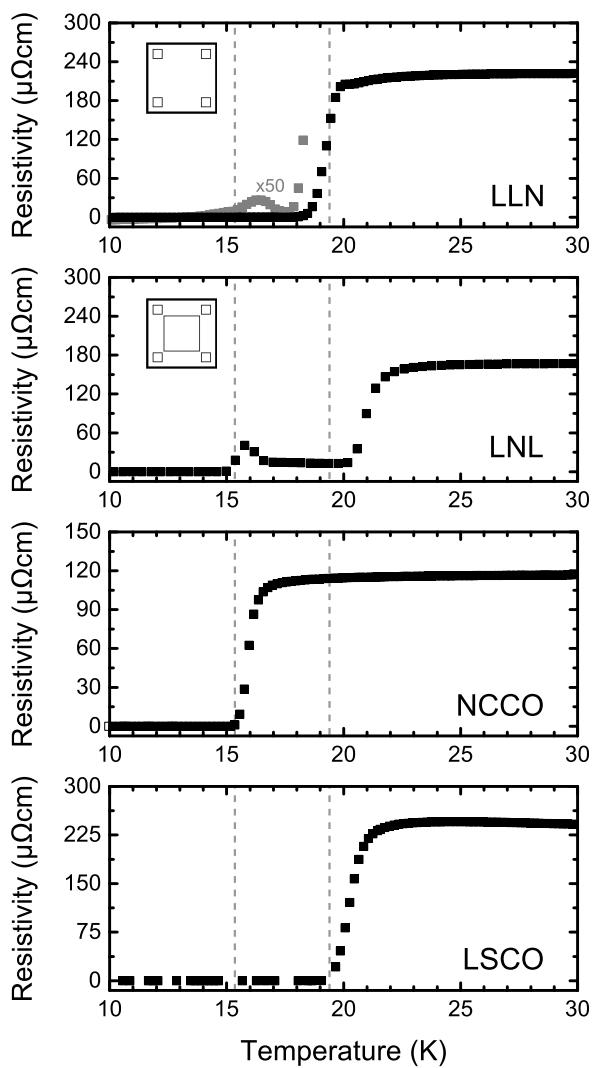

(b)
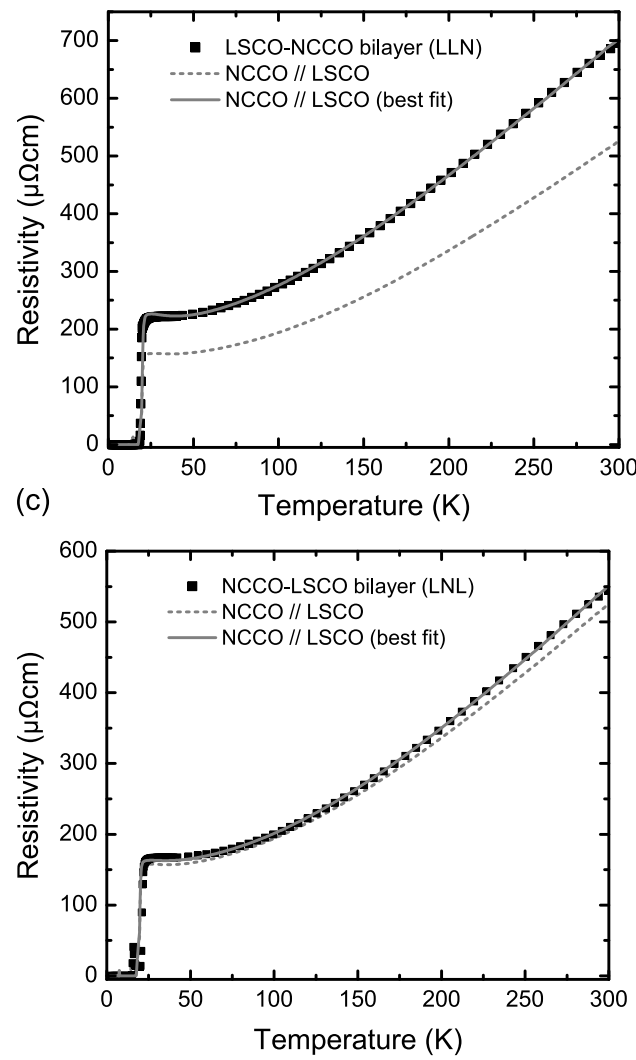

Figure 5.5: Resistivity measurements for the LNL and LLN configuration and a comparison to reference measurements on NCCO and LSCO. (a) Close up around $T_{c}$, from top to bottom: LLN, LNL, NCCO and LSCO. The van der Pauw measurement geometry for the LLN and the LNL configuration is indicated. The gray dashed lines show the $T_{c, 0}$ as measured in the reference samples. The LLN panel also shows a small feature for NCCO below the $T_{c}$ of LSCO, multiplied by 50 for clarity. (b) Resistivity for the LLN configuration compared to a parallel circuit of NCCO and LSCO (derived from the reference data), the dashed gray curve is for $150 \mathrm{~nm}$ thick layers, the solid gray curve is a least squares fit using scaling factors, yielding a relative additional conduction contribution of 2.27:1 (LSCO:NCCO). (c) Resistivity for the LNL configuration compared to the parallel circuit of NCCO and LSCO. The dashed gray curve is the same as in (b), the solid gray curve is a least squares fit using additional scaling factors, giving a relative conduction contribution of 1.18:1 (NCCO:LSCO). 
(a)

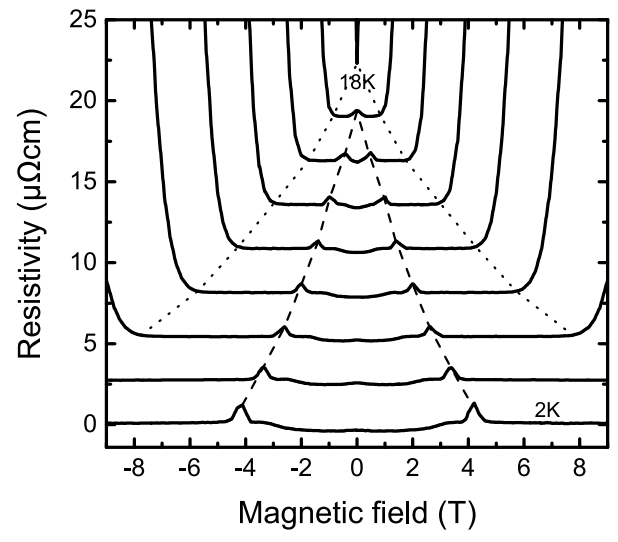

(b)

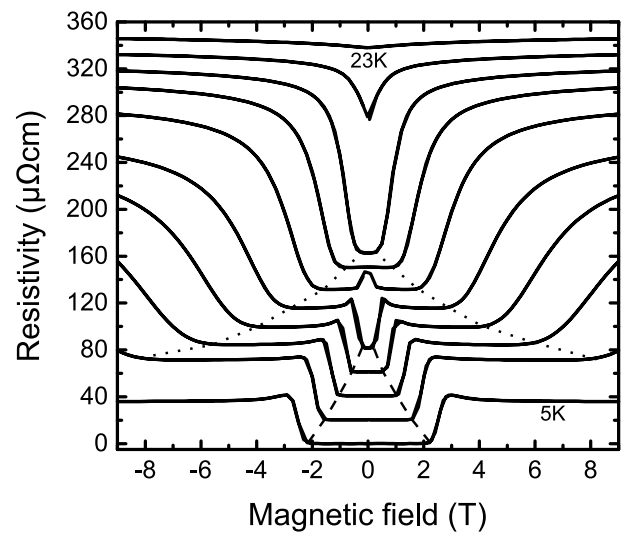

Figure 5.6: (a) Resistivity versus magnetic field for the LLN configuration, the curves are displaced for clarity. Temperature increases from bottom to top in $2 \mathrm{~K}$ increments from $2 \mathrm{~K}$ to $18 \mathrm{~K}$. (b) Resistivity versus magnetic field for the LNL configuration, the curves are displaced for clarity. Temperature increases from bottom to top in $2 \mathrm{~K}$ increments from $5 \mathrm{~K}$ to $23 \mathrm{~K}$. The dashed lines indicate features associated with $H_{c 2}$ of $\mathrm{NCCO}$ and the dotted lines correspond to $H_{c 2}$ of LSCO, as described in the main text.

an initial vacuum annealing showed nearly a factor of two higher resistance, while retaining more or less the same temperature dependence. The fit deviates from the measured data near the $T_{c}$ of LSCO; at these temperatures the interface barrier starts to play a role and the conductance ratio will shift in favor of the top layer, the NCCO, which does not show an upturn.

Figure 5.5(c) shows the temperature dependence of the resistivity for the LNL configuration. Here, the reference samples in parallel (dashed gray curve) underestimates the measured resistivity by a small amount, but the general shape of the curves is the same. The reference data can be scaled using the same procedure as used for the LLN measurement (solid gray curve). We find that the NCCO resistivity is nearly unaffected with only a $2 \%$ decrease and the LSCO resistivity increases by $17 \%$. When we assume an uncertainty in layer thickness for both the bilayer and the reference samples of around $5 \mathrm{~nm}$, the deviation for the NCCO layer falls within the error bar. The higher resistivity of the LSCO layer (or lower contribution to the conductivity) can be explained by increased scattering due to defects or voids originating in the NCCO layer, see figure 5.4, and by the measurement geometry favoring transport through the NCCO layer. It is interesting to note that we do not observe the same resistance increase for the NCCO as for the LLN configuration. This suggests that the LSCO layer can act as a capping or buffer layer against oxygenation of the NCCO.

We use magnetotransport measurements to verify that both the NCCO and the LSCO in the bilayers show superconductivity. Figure 5.6 shows the resistivity versus magnetic field for the LLN (a) and the LNL (b) configuration. Features 
corresponding to $\mathrm{H}_{\mathrm{c} 2}$ of $\mathrm{NCCO}$ and LSCO can be recognized for both configurations. The signal for NCCO is weaker for the LLN configuration, because below $T_{c}$ of LSCO there is only a small amount of transport through the NCCO layer (in the $c$-axis direction). If we assume that the peaks corresponding to $H_{c 2}$ for $\mathrm{NCCO}$ in (a) correspond to the peaks above $H_{c 2}$ in (b), we find that $H_{c 2}$ is very comparable between both configurations, with the LLN configuration giving a slightly larger value. This might be explained by the NCCO being deposited last in the LLN configuration and thus being exposed to less thermal cycling or by stronger pinning due to defects from strain accommodation. The reverse is seen for $H_{c 2}$ of LSCO, where we find a larger value for the LNL configuration, likely related to the density of the voids as pinning sites, observed in figure 5.4. The $H_{c 2}$ values for LSCO and $\mathrm{NCCO}$ found in the bilayer configurations are also comparable to what is found for the reference samples with $H_{c 2}$ of LSCO always a factor of 2-3 larger than $H_{c 2}$ of NCCO.

\subsection{2 c-axis transport}

Several configurations were considered to get independent contact to the $p$ and $n$ type layers. Unlike for the bilayer experiment in semiconductors [171], we cannot easily use gate tuning to deplete the top or bottom layer to get the independent contact. For full film bilayers and multilayers we have used careful etching to expose the bottom layer and then deposit gold contacts on both the bottom layer and the top layer. Wire bonding directly to the top layer invariably led to leakage currents between the two layers, even with insulating layers of e.g. $\mathrm{SrTiO}_{3}$ in between. For these devices we have had limited success with using small soft indium contacts to the top layer. This has led to unreliable contacts due to high contact resistances, size limitations, and the risk of penetrating through the layers is not fully eliminated. For smaller devices this technique is not very useful, mainly due to difficulty in scaling down the indium contacts.

In the final device fabrication we have employed passivation layers to lead the gold contacts from the top layer to the etched substrate surface for wire bonding, while avoiding contact with the bottom layer. As passivation layer we have used amorphous $\mathrm{SrTiO}_{3}$ and $\mathrm{Al}_{2} \mathrm{O}_{\mathrm{x}}$.

Junctions in the $c$-axis direction were fabricated from a LNL bilayer. The bilayer was structured into multiple mesa structures by argon ion milling. Part of each mesa structure was etched away to expose the bottom $\mathrm{NCCO}$ layer. $\mathrm{Au} / \mathrm{Ti}$ contacts are used to contact both layers for four probe measurements. For the top electrodes a layer of $\mathrm{AlO}_{\mathrm{x}}$ is first deposited around the edges of the structure by PLD to prevent direct contact to the bottom layer. The inset of figure 5.7(a) shows a schematic top view of the junctions.

Figure 5.7(a) shows an $I V$ measurement on the largest $c$-axis junction with a contact area of $620 \mu \mathrm{m} \times 630 \mu \mathrm{m}$ between the NCCO and the LSCO. Junctions with smaller contact area were also designed, but yielded no consistent results because of the large contact resistance to the LSCO layer. The contact resistance plays 
(a)

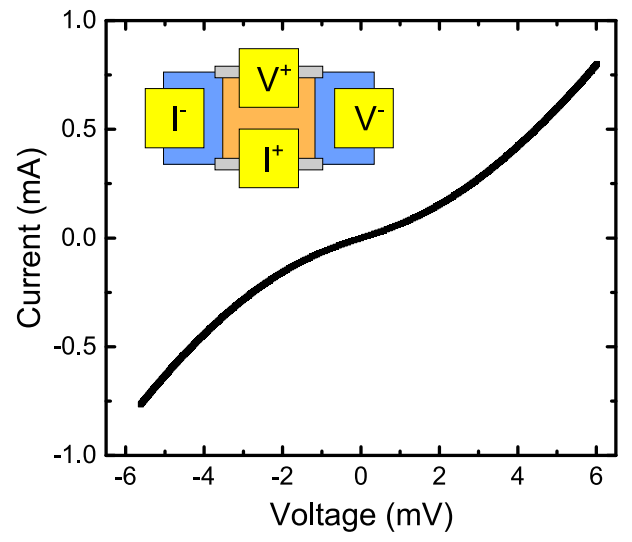

(b)

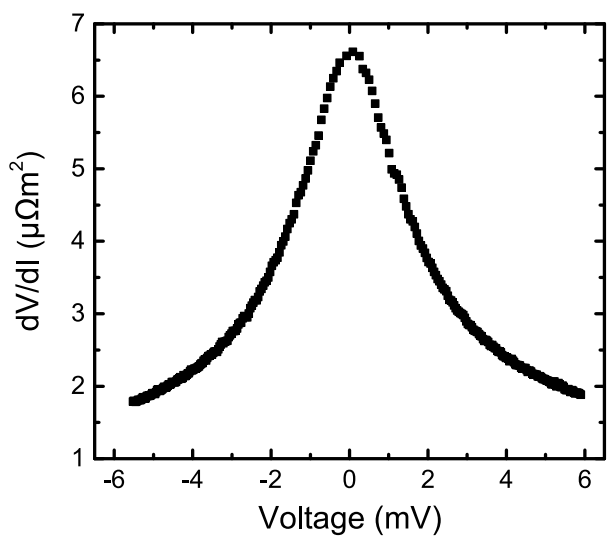

Figure 5.7: (a) $I V$ measurement on a $620 \mu \mathrm{m} \times 630 \mu \mathrm{m} c$-axis junction between NCCO and LSCO in the LNL configuration. The inset shows a schematic top view of the junction geometry. The blue area is ion milled down to the NCCO layer, the gray layers are $\mathrm{AlO}_{\mathrm{x}}$ that prevents contact between the top electrodes and the bottom layer. (b) $d V / d I$ characteristic normalized to junction area, numerically derived from (a).

a larger role in the $c$-axis contact compared to the in-plane junctions of chapter 3 , due to the limited contact area and the lack of any $(a, b)$-axis contact. This was discussed in $\S 1.2 .6$. For the junction of figure 5.7(a), the contact resistance limits the voltage that can be applied across the junction. Figure 5.7(b) shows the numerically derived resistance, normalized to the junction area. We can compare this to the results for the ramp-edge $p n$-junctions of chapter 3 . For the rampedge junctions we quote the resistance in $\Omega \mathrm{m}$. To get the same units as the $c$-axis junctions we have to multiply the resistance of the ramp-edge junctions by the thickness of the junction. As first approximation we can take this to be the thickness of the individual electrodes, $150 \mathrm{~nm}$, but one could also argue that this should be the length along the ramp, which is around $350 \mathrm{~nm}$ for a ramp angle of $26^{\circ}$. Using these two extremes, we find that the resistance of the $c$-axis junctions is 5-20 times larger than that of the ramp-edge $p n$-contacts.

Fitting the $I V$ characteristic of figure 5.7 with the asymmetric barrier tunneling model from chapter 3 has limited success. As was seen in chapter 3 , the model only captures the physics for higher bias; we ignore image force and density of states effects that modify the behavior for bias below the barrier. The barrier height from the model fit is of the order of $10-20 \mathrm{mV}$ with a barrier thickness of $10-15 \mathrm{~nm}$. The thickness of the barrier is larger compared to what we find for the in-plane contacts discussed in chapter 3 . This can be explained by the large conductance anisotropy found in cuprates like NCCO and LSCO [29]: conductivity in the $\mathrm{CuO}_{2}$ planes is one or two orders of magnitude larger than the $c$-axis conductivity. This is also reflected in the Thomas-Fermi screening length, which is larger in the c-axis direction, see chapter 1 . 
(a)

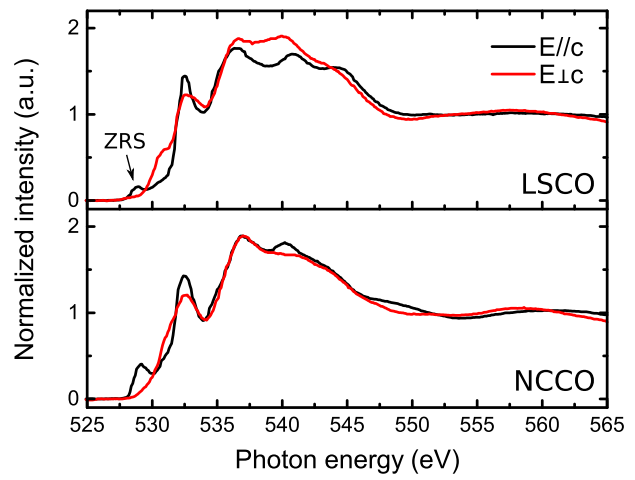

(b)

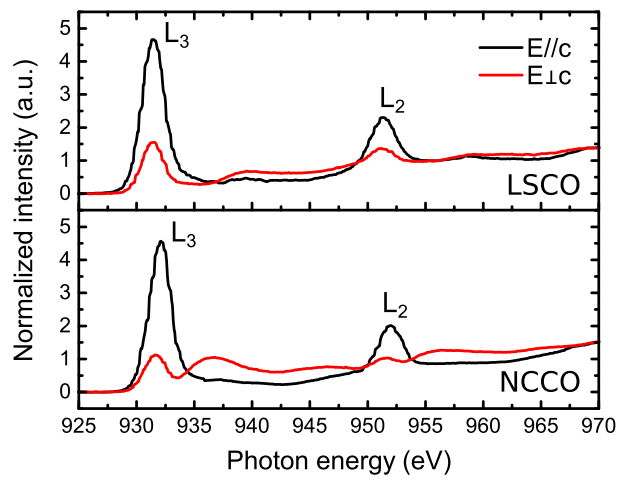

Figure 5.8: X-ray absorption spectra for $70 \mathrm{~nm}$ LSCO (top panels) and $70 \mathrm{~nm}$ NCCO (bottom panels) films on LSAT measured at the $\mathrm{O} K$-edge (a) and the $\mathrm{Cu} L_{2,3}$-edge (b) in $E / / c$ (black) and $E \perp c$ (red) configuration. The spectra are normalized to the background above the absorption edges.

\subsubsection{X-ray absorption spectroscopy}

X-ray absorption spectroscopy (XAS) measurements were performed in collaboration with the group of prof. Rusydi at the National University of Singapore at the Singapore Synchrotron Light Source (SSLS). Spectra were recorded near the O 1 s ( $K$-edge $)$ and the $\mathrm{Cu} 2 p\left(L_{2,3}\right.$-edge $)$ absorption edges. XAS probes the local electronic structure by exciting core electrons to unoccupied states, thereby gaining information on the unoccupied density of states, pre-edge exciton bound states and the electronic environment of the probed states through second order processes. The XAS transitions are determined by quantum mechanical selection rules and therefore electrons excited from different valence band orbitals will probe different final states. The XAS spectrum was recorded via the total electron yield with a probe depth of $\sim 50 \mathrm{~nm}$. The spectra were measured for two different polarizations, parallel and perpendicular to the $c$-axis, i.e. $E \perp c$ and $E / / c$, respectively. This was achieved by measuring with a normal incident beam for $E \perp c$ and at grazing incidence for $E / / c$.

Spectra were recorded for single NCCO and LSCO films of $70 \mathrm{~nm}$ thickness and a NCCO-LSCO bilayer, with NCCO as bottom layer and each layer $150 \mathrm{~nm}$ thick. The pre-edge intensity is set to zero and the spectra are normalized to the background intensity above the absorption edge. Due to the limited probe depth of XAS, only the top LSCO layer of the bilayer is probed in this experiment. The sample is the same as was used in the transport measurements of $\S 5.4 .1$, so the bottom NCCO layer was exposed by argon ion milling along the edges of the sample. In the XAS experiment, the beam is aligned to the center of the sample, to avoid the milled regions.

We compare our results to reference spectra for LSCO [172,173] and NCCO [173, 174]. Figure 5.8 shows the $\mathrm{O} K$-edge (a) and $\mathrm{Cu} L_{2,3}$-edge (b) spectra for the single 


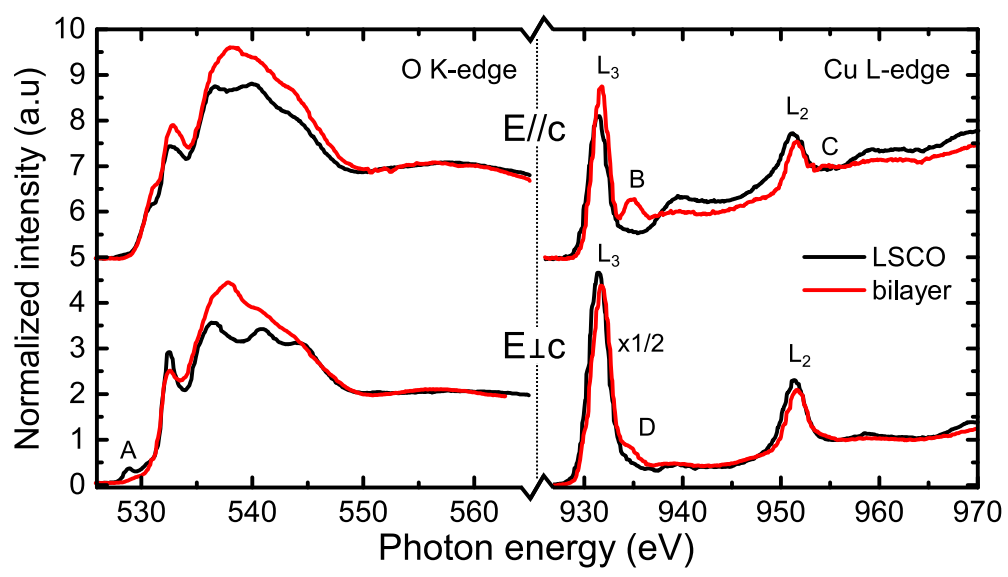

Figure 5.9: X-ray absorption spectra for $70 \mathrm{~nm}$ LSCO (black) and a $2 \times 150 \mathrm{~nm}$ NCCO-LSCO bilayer measured at the $\mathrm{O} K$-edge (left) and the $\mathrm{Cu} L_{2,3^{-}}$ edge (right) in $E / / c$ (top) and $E \perp c$ (bottom) configuration. The spectra are normalized to the background above the absorption edges. The $E \perp c \mathrm{Cu} L$-edge spectrum is multiplied by $1 / 2$. Peaks of interest are labeled.

layers of NCCO (bottom) and LSCO (top). There is a qualitative agreement for the NCCO spectra, all the features observed by Pellegrin et al. [173] are visible and the upper Hubbard band (UHB) edge starts around $528 \mathrm{eV}$. The spectra for LSCO are slightly different from the reference spectra of Pellegrin et al. [173]; the $E / / c \mathrm{O} K$-edge spectrum shows a more pronounced upper Hubbard band and the Zhang-Rice singlet (ZRS) charge transfer peak appears at about $0.5 \mathrm{eV}$ higher energy. The spectrum compares better to measurements on LSCO fims [172] than single crystals [173]. The peak positions are different for the (103) oriented films of Chen et al. [172], but the energy difference between the UHB and the ZRS is very similar to the energy difference between the peaks seen in figure 5.8(a). This might be an intrinsic difference between single crystals and films, where in the latter strain from the substrate can play an important role. It is interesting to note that the $E / / c$ spectrum does not show any signature of the ZRS peak, where this is observed in the reference spectra of Chen et al. [172] and Pellegrin et al. [173]. The $\mathrm{Cu} L_{2,3}$-edge show a better agreement with the reference data for LSCO. The only difference is a small peak around $940 \mathrm{eV}$ in the $E \perp c$ spectrum, which is caused by a slight $E / / c$ contribution, due to a minor misalignment in the measurement.

Figure 5.9 compares the $\mathrm{O} K$-edge (left panel) spectra and the $\mathrm{Cu} L_{2,3}$-edge spectra (right panel) for LSCO in the reference sample and in the NCCO-LSCO bilayer. The spectra show reasonable agreement, indicating that most of the signal of the bilayer is coming from the top LSCO layer, which is expected from the limited probe depth. No shift is observed for the $E / / c \mathrm{O} K$-edge spectrum, only a small increase in intensity at the UHB shoulder at $531 \mathrm{eV}$. This might be related to the larger thickness of the LSCO layer in the bilayer sample or signal from the exposed 
NCCO at the edges of the sample, which has significant UHB intensity already at $951 \mathrm{eV}$, see figure $5.9(\mathrm{a})$, lower panel. In the corresponding $E \perp c$ spectrum, the ZRS signature (A in figure 5.9) has completely disappeared in the bilayer, which might have been replaced by a broad, smeared-out peak at $\sim 1 \mathrm{eV}$ higher energy. Overall, with the exception of the UHB shoulder at $531 \mathrm{eV}$, the $\mathrm{O} \mathrm{K}$ edge spectra for the two different polarizations for the bilayer are very similar, indicating a smaller anisotropy in the LSCO layer in the bilayer. This is also reflected in the $\mathrm{Cu} L_{2,3}$-edge spectra, where the intensity ratio of the $L_{3}$ peak at $931.5 \mathrm{eV}$ is changed by a relative increase in intensity of the $E / / c$ signal. For both $E / / c$ and $E \perp c$, the broad feature around $940 \mathrm{eV}$ is suppressed and a pronounced peak has appeared at 935 and $934.5 \mathrm{eV}$, respectively. Again, these spectra show a more isotropic behavior. This could signal a more three-dimensional nature of the band structure [93], which might be related to the disappearance of the ZRS peak. However, the cross-over to a more three-dimensional occurs for tensile strain in LSCO [93] and from XRD we see no evidence of more strain in the bilayer structure as compared to the reference sample, see §5.3.1.

The peaks at $\sim 935 \mathrm{eV}$ (B and $\mathrm{D}$ in figure 5.9) in the $\mathrm{Cu} L_{3}\left(2 p_{3 / 2}\right)$ spectrum are a signature of nominally monovalent $\mathrm{Cu}^{1+}[175]$. This signature is characteristic for the $\mathrm{Cu}-\mathrm{O}$ chains in e.g. YBCO [176-178] and is also found in compounds like $\mathrm{Cu}_{2} \mathrm{O}$ [179]. The XAS spectrum also shows a small peak around $954.5 \mathrm{eV}$ (C in figure 5.9), which corresponds to the $\mathrm{Cu} L_{2}$ edge $\left(2 p_{1 / 2}\right)$ for $\mathrm{Cu}^{1+}$. We measure the splitting between the $\mathrm{Cu}^{1+} L_{2}$ and $L_{3}$ peaks to be $19.6(1) \mathrm{eV}$, close to what is measured e.g. for bulk $\mathrm{Cu}_{2} \mathrm{O}, 19.8 \mathrm{eV}$ [179]. The peak positions are close to what is observed for YBCO [178] and they are shifted to higher energy by around $1 \mathrm{eV}$ compared to $\mathrm{Cu}_{2} \mathrm{O}$ [179]. The latter can be explained by $\mathrm{Cu}_{2} \mathrm{O}$ having additional intensity below the edge due to an exciton mode.

The presence of $\mathrm{Cu}^{1+}$ should also be reflected in the $\mathrm{O} K$-edge spectra, since it is expected that the $\mathrm{Cu} 3 d$ and $4 s$ orbitals hybridize with nearby $\mathrm{O} 2 p$ levels. Other than the disappearance of the ZRS feature in the $E \perp c \mathrm{O} K$-edge spectrum, there are no obvious additional features near the UHB in figure 5.9, unlike what is observed for $\mathrm{YBCO}$ when $\mathrm{Cu}^{1+}$ is present in the $\mathrm{Cu}-\mathrm{O}$ chains [178]. The main difference is observed in the spectrum above the main edge, between 535 and $550 \mathrm{eV}$. The three peaks associated with the LSCO spectrum are present in the bilayer, but additional peaks are needed to explain the full shape of the spectrum for the bilayer. $\mathrm{Cu}_{2} \mathrm{O}$ has clear features at $538.6 \mathrm{eV}$ and $543.4 \mathrm{eV}[179,180]$, which fall between the peaks in the LSCO spectrum, creating the intensity continuum observed for the bilayer LSCO.

LSCO does not have a $\mathrm{Cu}-\mathrm{O}$ chain structure and $\mathrm{Cu}^{1+}$ is generally not observed in LSCO [172,173]. A likely candidate would be a parasitic inclusion of e.g. $\mathrm{Cu}_{2} \mathrm{O}$ in the NCCO-LSCO bilayer. However, no signature of $\mathrm{Cu}_{2} \mathrm{O}$ appears in the XRD spectrum of figure 5.3. The unknown peak in the spectrum, B in figure 5.3, cannot be linked to $\mathrm{Cu}_{2} \mathrm{O}$ [181]. Also, this unknown peak appears in both the bilayer and the reference sample used for the XAS measurements of figure 5.8, while only the bilayer shows a $\mathrm{Cu}^{1+}$ signature. Small features are observed for the $(002)$ and (004) diffraction peaks of $\mathrm{Cu}_{2} \mathrm{O}$, but they are too sharp to come from a 
$\mathrm{Cu}_{2} \mathrm{O}$ inclusion and turn out to come from $\mathrm{Cu} K \beta$ diffraction on LSAT (0 02$)$ and (004). It is not expected that the $\mathrm{Cu}^{1+}$ signature is related to the voids and the particles observed in figure 5.4, since they also appear in the reference samples. Furthermore, it is not originating from the NCCO; the underlying NCCO layer does not have any features related to $\mathrm{Cu}^{1+}$, as can be seen in figure 5.8(b) and from EDX we can rule out Ce diffusion into the LSCO as source of additional electrons. What remains is a change of $\mathrm{Cu}^{2+} / \mathrm{Cu}^{3+}$ to $\mathrm{Cu}^{1+}$ in the LSCO itself. Possible mechanisms for this might be oxygen vacancies, charge transfer from the NCCO, or a significant modification of the local $\mathrm{Cu}$ environment in the $\mathrm{CuO}_{2}$ planes. The last option is not very likely, since XRD has shown that the LSCO in the bilayer is very comparable to the reference sample and that the LSCO in the bilayer is relaxed more toward bulk values. That leaves oxygen vacancies or charge transfer, or a combination thereof. Both charge transfer effects and oxygen vacancies would have to come from the NCCO layer, since these features are not observed for single LSCO films. In the previous chapters we have shown that any electronic effects at the interface between NCCO and LSCO will only span a few nanometers. Since the XAS measurement is only probing the top of the LSCO layer, the charge transfer effect would have to extend much further than expected. Oxygen vacancies are therefore a more likely candidate, since they can diffuse throughout the film during the annealing procedure. They might also be the reason for the higher resistance of the LSCO layer observed in the transport measurements, figure 5.5(c). However, there is no evidence for vacancies migrating out of the NCCO layer: the LSCO actually appears to act as a buffer against oxygenation of the NCCO.

It would be interesting to investigate the distribution of the $\mathrm{Cu}^{1+}$ in the film, either by exploiting the probe depth difference between electron-yield mode and fluorescence-yield mode in XAS or by doing a depth profiling using X-ray photoelectron spectroscopy (XPS) combined with ion milling to probe the valence of the $\mathrm{Cu}$ throughout the layer.

\subsection{Conclusions}

We have developed a deposition scheme to fabricate NCCO-LSCO bilayers in two configurations, where both layers are superconducting. This allows the fabrication of multilayer/superlattice structures of importance to address interface effects in experiments. The interface in the $c$-axis direction is not sharp due to the intrinsic roughness of the NCCO layers. Further experiments towards interface effects like exciton formation requires more research into smoother growth of the NCCO layer and a decrease of the layer thickness to maximize the interface contribution. The interface structure of the LLN configuration was not investigated by HAADFSTEM, but it is expected to form a cleaner interface due to the layer-by-layer growth of the LSCO bottom layer.

In the LNL configuration we observe an electronic barrier at the interface that appears wider than observed for the in-plane contacts of chapter 3. The large contact resistance between the LSCO and gold contacts limits the measurement 
range. A more detailed study into the origin of the barrier and possible band alignment was not possible. Due to the two dimensional nature of the cuprates, the characteristic length scales of electronic effects are expected to be longer in the $c$-axis direction as compared to the $(a, b)$-axis direction, parallel to the $\mathrm{CuO}_{2}$ planes, see $\S 1.3 .5$.

X-ray absorption spectroscopy measurements were performed comparing the LNL configuration to reference films of LSCO and NCCO. Due to large layer thickness and limited probe depth in XAS, only the LSCO layer is measured in the LNL configuration. We find that the spectra for LSCO in the reference sample and the LNL bilayer agree globally with two major differences: the Zhang-Rice singlet peak, characteristic of hole doping in cuprates, has disappeared in the LNL configuration and a signature corresponding to $\mathrm{Cu}^{1+}$ is observed. The $\mathrm{Cu}^{1+}$ signal is not caused by any obvious impurity phase and is tentatively attributed to oxygen vacancies, but more research is needed to fully identify the origin and location of the $\mathrm{Cu}^{1+}$ in the LNL bilayer and to find out why the ZRS signature has disappeared. 

CHAPTER 6

\section{Towards a test for current quantization in type-II Bose-Mott insulators}

Current quantization has been predicted to occur in type-II Bose-Mott insulators. In this chapter, the implications of the theory are discussed and experiments are presented towards a test for current quantization in a candidate type-II Bose-Mott insulator, the pseudogap phase of $\mathrm{La}_{2-\mathrm{x}} \mathrm{Sr}_{\mathrm{x}} \mathrm{CuO}_{4}$. A $\mathrm{La}_{1.95} \mathrm{Sr}_{0.05} \mathrm{CuO}_{4}$ is sandwiched between two superconducting $\mathrm{La}_{1.85} \mathrm{Sr}_{0.15} \mathrm{CuO}_{4}$ electrodes in a ramp-edge geometry. Ring structures with a single junction are designed for flip-chip magnetization measurements using a SQUID, where the current quantization should quantize the magnetization. All layers and elements of the ring devices are characterized individually. Single junctions are measured and show a temperature dependence associated with variable range hopping, characteristic for the low temperature spin-glass phase of $\mathrm{La}_{1.95} \mathrm{Sr}_{0.05} \mathrm{CuO}_{4}$. At the lowest temperatures there is a cross-over to few impurity hopping, described by the Glazman-Matveev theory. The $I V$ characteristics of the junctions are dominated by a NIS contact at the ramp interface, with a low barrier and inelastic tunneling, superimposed on the non-linear conductance of the $\mathrm{La}_{1.95} \mathrm{Sr}_{0.05} \mathrm{CuO}_{4}$ layer. Several design optimizations are proposed for the experiment, as well as new measurement geometries.

\subsection{Introduction}

Duality is a powerful concept in condensed matter theory for finding solutions to strong coupling problems by exploring a dual system where coupling is weak. A well known example in the condensed matter community is the duality between anti-de Sitter string theory and conformal field theory (AdS-CFT) $[182,183]$. In this chapter we focus on a different kind of duality, which is centered around disorder. In a superfluid, disorder can be introduced in the form of topological defects, called vortices. Increasing disorder corresponds to the presence of more vortex/anti-vortex pairs in the superfluid. The duality exists in the idea to not 
describe the superfluid as something with weak coupling that becomes disordered, leading to a strong coupling phase, but rather as a system of vortices that becomes more ordered as the superfluid becomes disordered. The exact mathematical principles of this duality were formulated by Beekman and Zaanen [184]. They study the crossover from a bosonic superfluid by the quantum disorder of vortices via a quantum critical point to a Bose-Mott insulator state. The duality has led to a surprising prediction for superconductors (which are electrically charged superfluids). The duality predicts that where in a type-II superconductor the magnetic field can penetrate in the form of magnetic vortices, something similar should happen in the ordered vortex state, i.e. when the system has crossed over into the Bose-Mott insulator state. Beekman en Zaanen predict the existence of a type-II Bose-Mott insulator, where so called Mott vortices can appear. These vortices manifest themselves as lines of supercurrent inside the insulator. Just as in a type-II superconductor vortices can appear when the penetration depth is larger than the coherence length, here, a dual 'penetration depth', called the Mott proximity depth, has to be bigger than the (dis)order parameter of the Bose-Mott phase. The (dis)order parameter shrinks and the Mott proximity depth grows near the phase transition, so this is where one would expect to find Mott vortices. Figure 6.1 shows a schematic phase diagram for a type-II superconductor and a type-II Bose-Mott insulator, illustrating the duality.

In this chapter we will discuss experiments towards a test for current quantization in candidate type-II Bose-Mott insulator. The theoretical considerations in this chapter are based on the Ph.D. thesis of Beekman [185] and the work by Beekman and Zaanen [184]. Here, we limit ourselves to a discussion the main implications of the theory and we will discuss how it can be applied in an experiment. We will mainly focus on underdoped $\mathrm{La}_{2-\mathrm{x}} \mathrm{Sr}_{\mathrm{x}} \mathrm{CuO}_{4}$ as a candidate material to host the type-II Bose-Mott insulator phase. We estimate the typical size of critical parameters from the theory, discuss potential experiments to measure Mott vortices, show the first device fabrication and measurements towards these experiments, and give suggestions for future experiments.

\subsection{Theoretical background}

In short, the duality is as follows: in a type-II Bose-Mott insulator (super)current is expelled, but it can penetrate locally through a vortex line, just like magnetic fields are expelled from a type-II superconductor, but penetrate locally in the form of Abrikosov vortices. For the type-II Bose-Mott insulator an applied current is the dual equivalent of the applied magnetic field for type-II superconductors. Figure 6.1 illustrates the equivalent phase diagrams for a type-II superconductor and a type-II Bose-Mott insulator. How a current can be applied to an insulator in an actual experiment, will be addressed later.

A natural candidate material for the Bose-Mott phase is the potentially preformed pair phase in the high- $T_{c}$ cuprates in the so-called pseudogap regime [184]. In this regime, a gap in the single-particle spectrum, similar to the superconducting gap 

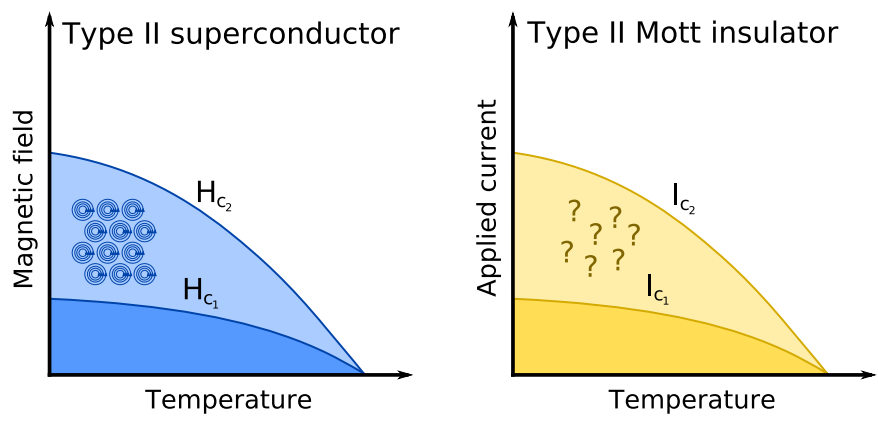

Figure 6.1: Schematic phase diagram for a type-II superconductor and a type-II Bose-Mott insulator, illustrating the duality. Abrikosov vortices appear in a type-II superconductor above a critical field $H_{c 1}$; in the type-II Bose-Mott insulator, Mott vortices are predicted to appear above a critical applied current.

is observed above $T_{c}$ and/or for low doping. In this phase there is no long-range superconducting order [186]. The origin of the gap-like features is not completely understood and is attributed to spontaneous symmetry breaking effects like spinand/or charge-density waves, or preformed Cooper pairs with no long range order, or both [186-189]. The latter explanation is a prime example of a Bose-Mott insulator candidate. Here, we are dealing with a phase disordered system of bosons at low excitation energies. This is exactly the kind of phase disorder transition described by Beekman et al. [184], including experimental evidence for vortices as topological defects of this phase [190-192]. The description of the pseudogap phase as a bad insulator is an extra indication that disorder plays an important role. An experiment on these systems works twofold; experimental observation of Mott vortices would both provide strong evidence for the existence of the type-II Bose-Mott insulator phase and preformed pairs in the pseudogap phase.

The main parameter that governs the transition from a superconductor to a BoseMott insulator is the quantum disorder, which is determined by the ratio $U / J$, where $U$ is the on-site Coulomb repulsion and $J$ is the hopping parameter in the Bose-Hubbard Hamiltonian. Figure 6.2 shows the full phase diagram as a function of temperature, applied field/current and quantum disorder [184]. The limit of $U / J \rightarrow \infty$ corresponds to the atomic case of the Mott insulator phase, where no perturbation is possible. Lowering $U / J$ brings the system into a regime where it transitions from a type-I to a type-II Bose-Mott insulator. Here, Mott vortices can appear as current lines above a critical current $I_{c 1}$. The corresponding phase diagram is shown in the right panel of figure 6.1. Upon further lowering of $U / J$ the system crosses the quantum critical point into the superconducting phase.

In the cuprates, the ratio $U / J$ is controlled by the chemical potential and thus to a certain extent by the doping level. As stated before, the highest chance of finding Mott vortices is where the Bose-Mott insulator order parameter is small, i.e. close to the transition into the superconducting phase. This is also the regime 


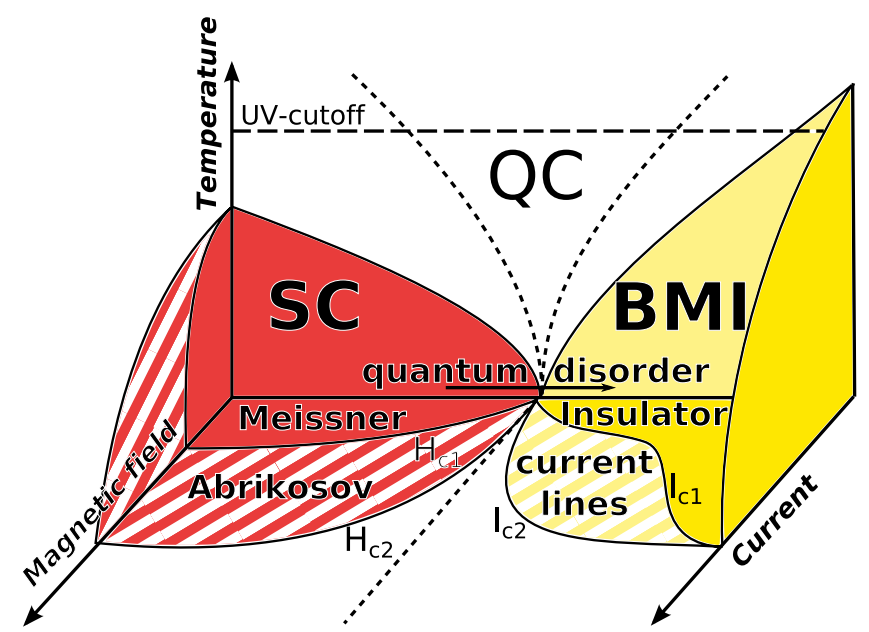

Figure 6.2: Three-dimensional phase diagram as function of temperature, applied field/current and quantum disorder $U / J$. Adapted from [184].

where the preformed pairs are expected (if they exist). Moving away further from the superconducting transition, the cuprates will revert to the fermionic Mott insulator state (the charge-transfer insulator at zero doping).

Important parameters for an experiment to measure the predicted Mott vortices are the Mott proximity depth $\lambda_{M}$, the magnitude of the current quantized in the Mott vortices $I_{0}$, and to a lesser extend the dual coherence length $\tilde{\xi}$ and the order parameter for the Bose-Mott insulator $\Phi_{\infty}$ in the dual London limit (no amplitude fluctuations). The Mott proximity depth is given by

$$
\lambda_{M}{ }^{-1}=\sqrt{\frac{\hbar}{c_{p h} m^{*}} \rho_{s} \Phi_{\infty}^{2}},
$$

where $\hbar$ is Planck's constant, $m^{*}$ is the effective mass, $c_{p h}$ is the phase velocity, $\rho_{s}$ is the superfluid density and $\Phi_{\infty}$ is the Bose-Mott order parameter in the dual London limit. The superfluid density corresponds to the density of Cooper pairs, as there is no real superconducting order in the Bose-Mott phase.

The current quantum is given by

$$
I_{0}=\frac{(2 \pi)^{2}}{\Phi_{0}} \sqrt{U J}=\frac{e^{*} c_{p h}}{a} 2 \pi
$$

where $\Phi_{0}=\hbar / e^{*}$ is the magnetic flux quantum, $e^{*}$ is $2 e$ in the case of Cooper pairs, and $a$ is the lattice parameter. The first part of the equation illustrates the duality by the appearance of the inverse of the magnetic flux quantum. Unlike flux 
quantization in type-II superconductors, the current quantum is not a universal property, but it depends on material properties, both when defined as current or as current density using the Mott proximity depth. Here we have used the relation $c_{p h}=\frac{a}{\hbar} \sqrt{U J}$ to introduce the material properties expressed via $U$ and $J$ in the theory. To get an estimate for the size of the current quantum in a cuprate like $\mathrm{La}_{2-\mathrm{x}} \mathrm{Sr}_{\mathrm{x}} \mathrm{CuO}_{4}$ we can take the Fermi velocity as an approximation for the phase velocity, which is around $2.7 \times 10^{5} \mathrm{~ms}^{-1}$ for $\mathrm{La}_{2-\mathrm{x}} \mathrm{Sr}_{\mathrm{x}} \mathrm{CuO}_{4}$ and is only weakly dependent on the doping level [134]. Using a lattice parameter of $\sim 4 \AA$ in the $\mathrm{CuO}_{2}$ planes, we get a current quantum of $1.4 \mathrm{~mA}$.

We can take a similar approach to estimate the Mott proximity depth, to get a measure for the dimensions of the current filaments. This gives important size restrictions for the design of the measurement. Parallel to a magnetic vortex in a type-II superconductor, a Mott vortex is characterized by a core of the size of the coherence length and a decaying current over the Mott proximity depth. The current density inside the Mott vortex is roughly inversely proportional to the Mott proximity depth squared. The typical critical current density for LSCO used in this work is $1 \times 10^{9} \mathrm{~A} \mathrm{~m}^{-2}$; taking this as a limit for the current density in a Mott vortex leads to a Mott proximity depth larger than a micrometer.

The Mott proximity depth can also be estimated from equation 6.1. It can be rewritten, incorporating the London penetration depth $\lambda_{L}=\sqrt{m^{*} / \mu_{0} \rho_{s} e^{2}}$, giving

$$
\lambda_{M}=\lambda_{L}\left(\frac{c_{p h} \mu_{0} e^{2}}{\hbar \Phi_{\infty}^{2}}\right)^{1 / 2}
$$

Using $c_{p h}=2.7 \times 10^{5} \mathrm{~ms}^{-1}$ [134] and taking $\Phi_{\infty}^{2}$ to be close to 1 [185], we get a Mott penetration depth that is about $1 \%$ of the London penetration depth associated with the Bose-Mott insulator phase. This requires the London penetration depth to be at least $100 \mu \mathrm{m}$ to ensure the current quantum can actually be carried by the superfluid inside the Mott vortex. This is not an unreasonable value for $\lambda_{L}$, as it is expected to diverge towards underdoping; this is supported by e.g. the $c$-axis penetration depth for $\mathrm{La}_{1.92} \mathrm{Sr}_{0.08} \mathrm{CuO}_{4}$ being around $25 \mu \mathrm{m}$ [193]. The size of the Bose-Mott order parameter is taken to be at least unity, following Beekman et al. [184], and it can be seen from equation 6.3 that a larger value would quickly shrink $\lambda_{M}$. An experiment would have to look for a sweet spot where $\lambda_{L}$ is sufficiently large, but where $\Phi_{\infty}^{2}$ is still relatively small. This is another reason to look for Mott vortices close to the superconducting transition, where $\lambda_{L}$ is already diverging and the Bose-Mott order parameter is small.

It is important to realize that the values mentioned above are crude estimates. The theory describes the type-II Bose-Mott insulator as a more or less isotropic system in three dimensions whereas the cuprates are far from isotropic. In particular, a material like $\mathrm{La}_{2-\mathrm{x}} \mathrm{Sr}_{\mathrm{x}} \mathrm{CuO}_{4}$ has a layered two-dimensional character $[93,94]$. However, it is preferred over a more three-dimensional cuprate material like $\mathrm{YBa}_{2} \mathrm{Cu}_{3} \mathrm{O}_{7-\mathrm{x}}$ because of the fine control over the doping level through the strontium doping. 
Another reason for choosing $\mathrm{La}_{2-\mathrm{x}} \mathrm{Sr}_{\mathrm{x}} \mathrm{CuO}_{4}$ as a candidate type-II Bose-Mott insulator, is the observation of a giant proximity effect in a multilayer stack where an underdoped layer was sandwiched between two optimally doped $\mathrm{La}_{2-\mathrm{x}} \mathrm{Sr}_{\mathrm{x}} \mathrm{CuO}_{4}$ layers [194]. Here, the underdoped layer remained superconducting above its critical temperature through a giant proximity effect from the optimally doped layers over distances larger than expected from the coherence length in the $c$-axis direction. The underdoped layer was shown to be fully superconducting through measurement of the Meissner state [195]. A potential explanation for the observed effects is the occurrence of Mott vortices in the underdoped layer as a candidate type-II Bose-Mott insulator [184].

All this has motivated us to use $\mathrm{La}_{2-\mathrm{x}} \mathrm{Sr}_{\mathrm{x}} \mathrm{CuO}_{4}$ as a candidate material to set up an experiment to more directly measure the current quantization associated with the formation of the Mott vortices. A very basic question that should arise for any experimentalist in all this is: how does one apply a current to an insulator? In an actual experiment a voltage is applied and a current will flow due to this applied voltage. It is difficult to imagine how one could get the current to act like the magnetic field for a type-II superconductor. One solution is to let the applied current result from an applied phase difference by placing the type-II Bose-Mott insulator material as a junction in a superconducting ring. Applying a magnetic field to the ring will impose a phase difference across the type-II BoseMott insulator, which acts as our dual applied magnetic field. The nature of the type-II Bose-Mott insulator should become visible in the magnetization of the ring, which should reflect the quantized nature of the current quanta carried by the Mott vortices, i.e. the (super)current lines. This can be measured using a SQUID (superconducting quantum interference device). Ignoring any screening effects, the field required for a single Mott vortex to appear is comparable to the earth magnetic field for a ring size of $\sim 30 \mu \mathrm{m}$. The advantage of this approach is that there is no potential difference associated with the phase difference, which suppresses normal quasi particle transport through the type-II Bose-Mott insulator. This approach does require that a phase difference can actually be created across the type-II Bose-Mott insulator. In principle, this would require phase coherence across the type-II Bose-Mott insulator, making it more like a Josephson weak link. However, once the first Mott vortex is established, there is a phase coherence across the type-II Bose-Mott insulator junction.

Another possibility is to measure the current quantization in a direct measurement. Simply put, a layer of a type-II Bose-Mott insulator should show quantized conductance that increases with each quantum of applied current. However, if the type-II Bose-Mott insulator is a perfect insulator (apart from the Mott vortices), there is no clear way how the system could go from an applied current of $n I_{0}$ to $(n+1) I_{0}$. When we take a simple junction of a type-II Bose-Mott insulator sandwiched between two electrodes like a parallel plate capacitor, dc current bias is not possible. In a circuit, the type-II Bose-Mott insulator junction will dominate the resistance and result in a voltage bias across the junction, not a current bias. One can imagine that there exists a threshold voltage for which the charging energy of the capacitor setup, $C V^{2} / 2$, where $C$ is the junction capacitance, 
exceeds the energy required to nucleate a single Mott vortex. When the vortex nucleates, there is no voltage drop across the junction any more; the voltage drop is now across any resistance in series with the junction. Increasing the voltage further would normally result in an increase in current, but the junction will block any current exceeding the current associated with the current number of Mott vortices. Therefore, voltage will start to build up across the junction again, until the nucleation energy for the next vortex is exceeded. This suggests that the properties of the type-II Bose-Mott insulator junction can be explored by simple $I V$ measurements, but also in the time and frequency domain by changing the RC time associated with the circuit.

In a realistic system, the charging energy may not be the dominating factor. Next to the bosonic transport, a candidate system like LSCO will also show quasi particle transport. This means that the junction should be viewed as a parallel circuit of the idealized type-II Bose-Mott insulator in parallel with a shunt resistance. In fact, this could also be done by adding an actual shunt resistor to the circuit. In this case, current bias is possible, since below $I_{0}$, the shunt resistance will dominate transport and current can be increased continuously. Above $I_{0}$ applied current, energy minimization suggests that a Mott vortex should nucleate in the type-II Bose-Mott insulator part of the junction, resulting in a sharp drop in the voltage across the junction. Additional current will flow through the shunt resistance, until the next current quantum is reached. A current-voltage characteristic would look like a sawtooth pattern with a rising slope followed by a sharp drop.

In the following we will focus on both a ring like structure with a type-II Bose-Mott insulator junction and isolated type-II Bose-Mott insulator junctions.

\subsection{Experimental details}

A ring-like structure is not easy to fabricate for a $c$-axis junction like the system used by Bozovic et al. [194] as there are no reliable high-temperature lift-off techniques, which is necessary to achieve a structure with only one type-II BoseMott insulator junction. Therefore, we have chosen to fabricate a ring with an inplane superconductor/type-II Bose-Mott insulator/superconductor junction, using the ramp-edge structures described in chapter 3. The design of the full chip has seen several iterations where the design was optimized first for magnetization measurements to observe the current quantization in rings containing a type-II Bose-Mott insulator junction (MottSQUIDs) and later for transport measurements on individual junctions. The intended experiment is the measurement of the magnetization of a MottSQUID loop using a regular SQUID with a pickup loop in a flip-chip arrangement with the measurement SQUID on top of the MottSQUID device. The outline of the final design is shown in figure 6.3(c). For the flipchip experiments, the allowed overlap between the two chips is small due to the placement of the contact pads on the measurement SQUID. To avoid damage to either chip, the rings containing a Bose-Mott junction, the MottSQUIDs, are 
spread around the edges of the sample. The size of the rings is designed to be comparable to that of the pick-up loops of the measurement SQUID. Initial designs still had measurement leads to the MottSQUIDs, but they were removed in later designs as their usefulness was minimal and they caused stray signals in the measurement SQUID. The measurement leads were left for a few rings, since the ring structure also provides a natural shunt resistance in parallel with the type-II Bose-Mott insulator junction, suitable for direct transport measurements. The middle of the chip is filled with test structures for the individual layers, the superconducting short, a set of five individual junctions and a final set of MottSQUID loops with and without measurement leads. The MottSQUID loops in the center are not immediately accessible in the flip-chip measurements. In general, the center of the sample has the highest quality film growth and in the case of $\mathrm{La}_{2-\mathrm{x}} \mathrm{Sr}_{\mathrm{x}} \mathrm{CuO}_{4}$ is the closest to the target composition in $\mathrm{Sr}$ doping ${ }^{\dagger}$. Therefore, this area was also reserved for MottSQUID loops that could be accessed for measurement by cutting the chip if necessary.

The sample fabrication process is schematically depicted in figure 6.3(a). The final devices consists of three sets of layers, the first two define the Bose-Mott junction (panels 1 and 2) and the third layer closes the ring structure with a superconducting contact (panel 4). All $\mathrm{La}_{1.85} \mathrm{Sr}_{0.15} \mathrm{CuO}_{4}$ and $\mathrm{La}_{2} \mathrm{CuO}_{4}$ layers are deposited by PLD at $700^{\circ} \mathrm{C}$ and 0.13 mbar oxygen pressure and subsequently annealed using the standard annealing procedure for $p$-type cuprates in 1 bar oxygen with dwell periods of $15 \mathrm{~min}$ and $30 \mathrm{~min}$ at $600{ }^{\circ} \mathrm{C}$ and $450{ }^{\circ} \mathrm{C}$, respectively. First, a multilayer of $50 \mathrm{~nm} \mathrm{La} 2 \mathrm{CuO}_{4}, 100 \mathrm{~nm} \mathrm{La}{ }_{1.85} \mathrm{Sr}_{0.15} \mathrm{CuO}_{4}$ and $20 \mathrm{~nm} \mathrm{La}_{2} \mathrm{CuO}_{4}$ is deposited on a $\left[\mathrm{LaAlO}_{3}\right]_{0.3}\left[\mathrm{Sr}_{2} \mathrm{AlTaO}_{6}\right]_{0.7}$ (LSAT) substrate. The initial $\mathrm{La}_{2} \mathrm{CuO}_{4}$ layer will act as a buffer layer for the subsequent LSCO layers, as was discussed before in chapter 3 , where $\mathrm{Nd}_{2} \mathrm{CuO}_{4}$ was used. The top layer acts as a capping layer to avoid any $c$-axis contact between the two electrodes. This multilayer is structured ex situ into the first half of the ring structure by argon ion milling, panel 1 in figure 6.3(a). The ion milling is done under an angle of $45^{\circ}$ to create two ramps at the ends of the half ring. The ring has an asymmetric shape; one side has a wider ramp to ensure a fully superconducting contact with the subsequent layers for a high critical current of the final device. Next, we deposit $50 \mathrm{~nm}$ of underdoped $\mathrm{La}_{1.95} \mathrm{Sr}_{0.05} \mathrm{CuO}_{4}$ as the candidate type-II Bose-Mott insulator layer and $100 \mathrm{~nm}$ of $\mathrm{La}_{1.85} \mathrm{Sr}_{0.15} \mathrm{CuO}_{4}$ after an in situ soft argon ion milling to clean the interface. These two layers are structured into the second part of the ring device by argon ion milling under $45^{\circ}, 180^{\circ}$ rotated with respect to the previous etching to create two opposing ramps where the final layer will join the two sides of the ring structure, see panel 2 in figure 6.3(a). Before the final layer is deposited, the capping layer on the first electrode is removed by ion milling, to create a $c$-axis contact to the final layer, see panel 3 in figure 6.3(a). The last layer of $150 \mathrm{~nm}$ of $\mathrm{La}_{1.85} \mathrm{Sr}_{0.15} \mathrm{CuO}_{4}$ is again deposited after an in situ cleaning step using soft argon ion milling. Finally, the last layer is structured by argon ion milling, removing the last layer everywhere except in the overlap area of the superconducting short, see panel 4 in figure 6.3(a)

\footnotetext{
${ }^{\dagger}$ La tends to spread out more than Sr in the PLD plume, due to the atomic weight difference between $\mathrm{Sr}$ and $\mathrm{La}$, resulting in a decrease of the $\mathrm{Sr} / \mathrm{La}$ ratio towards the edge of the sample.
} 
(a)

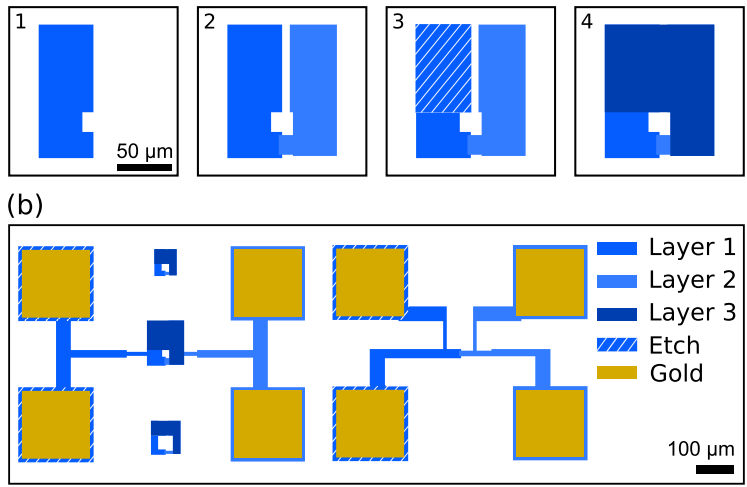

(c)

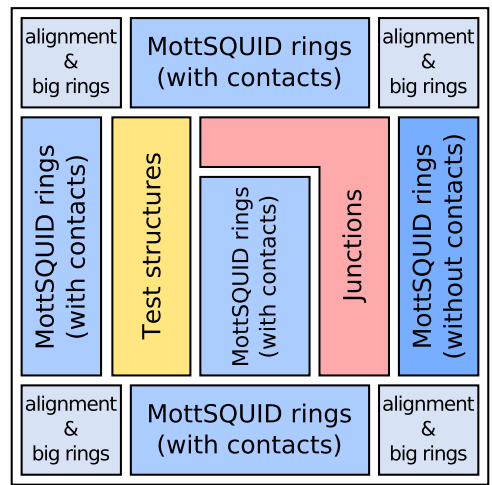

Figure 6.3: Layout of the sample for flip-chip magnetization measurements and on-chip transport measurements. (a) Schematic fabrication process for a MottSQUID loop: (1) $\mathrm{La}_{2} \mathrm{CuO}_{4}-\mathrm{La}_{1.85} \mathrm{Sr}_{0.15} \mathrm{CuO}_{4}-\mathrm{La}_{2} \mathrm{CuO}_{4}$ stack defined into bottom electrode structure by argon ion milling; (2) $\mathrm{La}_{1.85} \mathrm{Sr}_{0.05} \mathrm{CuO}_{4}$ $\mathrm{La}_{1.85} \mathrm{Sr}_{0.15} \mathrm{CuO}_{4}$ second electrode definition, makes the Bose-Mott junction; (3) $\mathrm{La}_{2} \mathrm{CuO}_{4}$ capping layer is removed in the dashed area; (4) final $\mathrm{La}_{1.85} \mathrm{Sr}_{0.15} \mathrm{CuO}_{4}$ layer to close MottSQUID loop. (b) Top view of typical designs for the junctions and the MottSQUID loops with and without electrodes. (c) Layout of the devices on the chip, the MottSQUID loops are spread out along the edges of the sample; junctions and test structures are in the center of the sample.

After each etching step the etch depth and the ramp profile was measured using atomic force microscopy (AFM). Figure 6.4 shows a typical result for the ramp in the first layer (a) and an overview of a finished ring design (b).

Transport measurements on all structures of the design in figure 6.3 were performed in three different systems. Basic transport characterization was done in a Quantum Design 9 T Physical Property Measurement System (PPMS). Resistance versus temperature measurements were performed using low bias currents in zero field and $9 \mathrm{~T}$. $I V$ measurements up to $5 \mathrm{~mA}$ were performed using the AC transport option of the PPMS. Similar measurements were also performed in a magnetically shielded bath cryostat, where the sample is contained in a superconducting $\mathrm{Nb}$ shield and in a flow cryostat with a BSCCO pot for shielding. Both setups are also equipped to apply $\mathrm{RF}$ radiation to the sample. In these two systems the measurements are performed using home-built differential amplifiers and a triangle generator (a home-built version and a HP 3312A function generator) with passive low-pass filters on the measurement leads. A major hurdle in the measurements is the large non-linear contact resistance found between gold and LSCO. This requires large input resistances on the measurement side to prevent current leakage through the measurement circuit. The differential amplifiers work with FET input amplifiers that have G $\Omega$ input resistances, ensuring low leakage as these resistances are several orders of magnitude larger than the resistance of the junctions and MottSQUIDs, including the contact resistances. The details of 
(a)

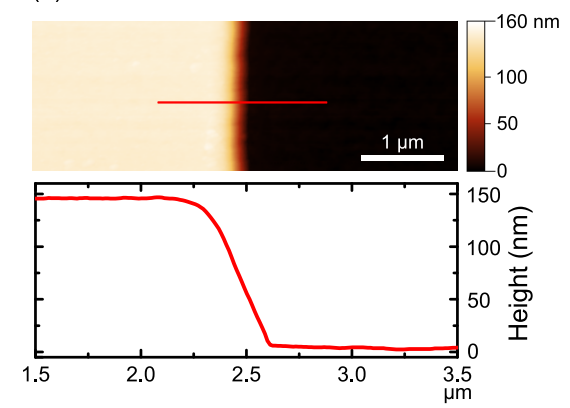

(b)

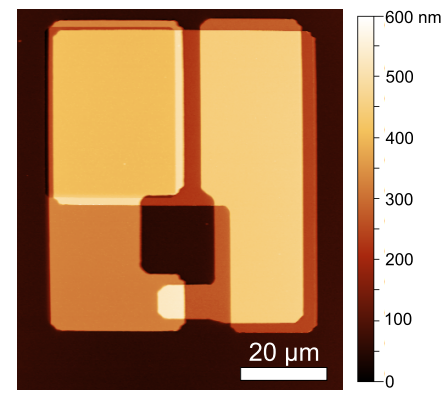

Figure 6.4: AFM images of a MottSQUID device. (a) topography map of the ramp etched into the first layer and a profile (red line in top panel) across the ramp. (b) AFM overview of a finished MottSQUID device, the layers from figure 6.3(a) can be recognized. The final layer is slightly misaligned.

the measurement circuit in the PPMS are not reported by Quantum Design, but it appears to have input resistances similar to the differential amplifiers. The general behavior of the junctions is the same for measurements in both systems. The main deviation is found in the way the $I V$ measurements are performed. In the PPMS the $I V$ measurements are performed quasi dc, where the current is incremented in steps and stabilized before the voltage is measured. In the cryostats the $I V$ characteristics are recorded using a low frequency sawtooth and continuous voltage sampling. The data is numerically averaged by a binning algorithm that collects the voltage measurements in current bins. The system has a high characteristic $R C$ value, due to the high contact resistance, combined with the sample capacitance and the capacitors in the low pass filters. This is reflected in an opening up of a hysteresis in the $I V$ characteristics for higher sawtooth frequencies $(>1 \mathrm{~Hz})$. The sawtooth measurements are only reliable for low bias. At the critical current of the LSCO leads, the current source cannot keep a constant output voltage due to the large load caused by the extra resistance of the LSCO leads. This causes measurement artifacts such as apparent excess current and rounding of the $I V$ curves. In the PPMS this issue is avoided by operating the system in the high impedance/constant current-mode, where the source impedance is $>10 \mathrm{M} \Omega$. However, the PPMS has a limited voltage resolution for the low bias regime. Examples of these effects and more details can be found in appendix A.

\subsection{Sample characterization}

Quality control of the individual layers is important as the sample is cycled three times through high temperature and low (deposition) pressure, both of which can deteriorate the quality of the layers by oxygen off-stoichiometry and partial decomposition. The three sets of layers in the MottSQUID design are measured separately in the final device through test structures corresponding to the layers in 
(a)

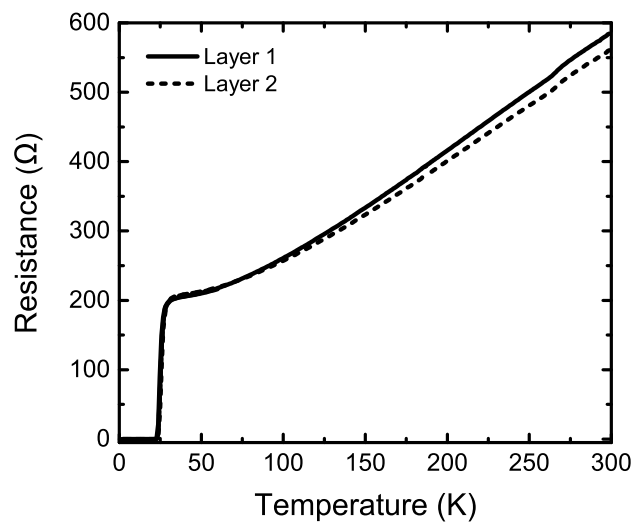

(b)

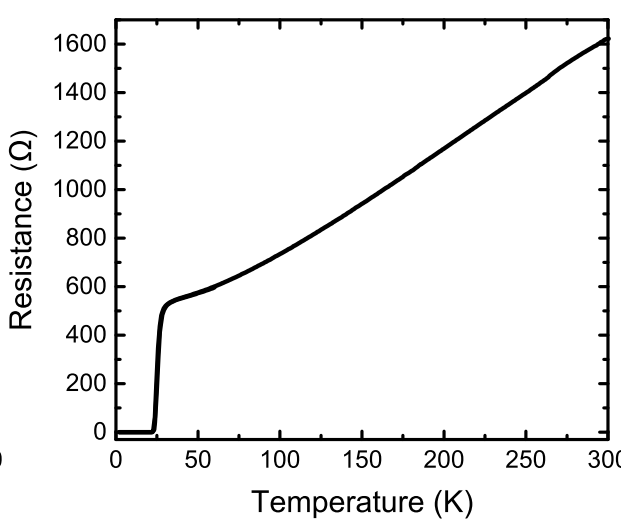

Figure 6.5: Resistance versus temperature measurements on the test structures on the MottSQUID chip for the three layers of the device: (a) layer 1 (solid curve) and layer 2 (dashed curve), and (b) layer 3, note the different scale.

panels 1, 2 and 4 in figure 6.3(a). Figure 6.5 shows resistance versus temperature for the three test structures. The first and second layer (a) show the same general shape of the resistance curve and only a small difference in total resistance. The resistance of the third layer (b) is a factor of three higher than the other two, but again the general shape is the same. We attribute the higher resistance of the third layer to the roughness of the nucleation layer. The third layer is grown on a surface where the previous two LSCO layers have been etched away and where only a thin, rough $\mathrm{La}_{2} \mathrm{CuO}_{4}$ buffer layer remains. This is reflected in the resistance of the layer, but not in the $T_{c}$; all layers show the same critical temperature, within $0.5 \mathrm{~K}$ of each other, of $22.3 \mathrm{~K}$. We find a critical current density of $7.4 \times 10^{8} \mathrm{~A} \mathrm{~m}^{-2}$ for the first layer and $6.5 \times 10^{8} \mathrm{~A} \mathrm{~m}^{-2}$ for the second layer. Both layers show a higher critical current density than the LSCO layers of the $p n$-junctions discussed in chapter 2. We attribute this to the better lattice match of the $\mathrm{La}_{2} \mathrm{CuO}_{4}$ buffer layer as opposed to the $\mathrm{Nd}_{2} \mathrm{CuO}_{4}$ layer used in the $p n$-junctions of chapter 2 . The critical current density is lower than what we find for the LSCO-LSCO homojunctions, also discussed in chapter 2. This can be explained by the thicker layers used for the homojunctions and the additional deposition step for the third layer in the MottSQUID sample. Compared to the predicted current quantum of $\sim 1.4 \mathrm{~mA}$, the critical current of the leads for most of the junctions in the design is of the same order of magnitude, i.e. $1-2 I_{0}$.

A final test structure is used for measuring the superconducting short that is closing the MottSQUID rings, the top section of the ring if figure 6.3(a). Figure 6.6 shows a resistance versus temperature measurement for this structure. The resistance measurement shows the same general behavior as was seen for the individual layers, but a finite resistance remains below $T_{c}$. The inset of figure 6.6 shows that at $T_{c}$ the resistance first drops to around $1 \Omega$ and then slowly decreases 
(a)

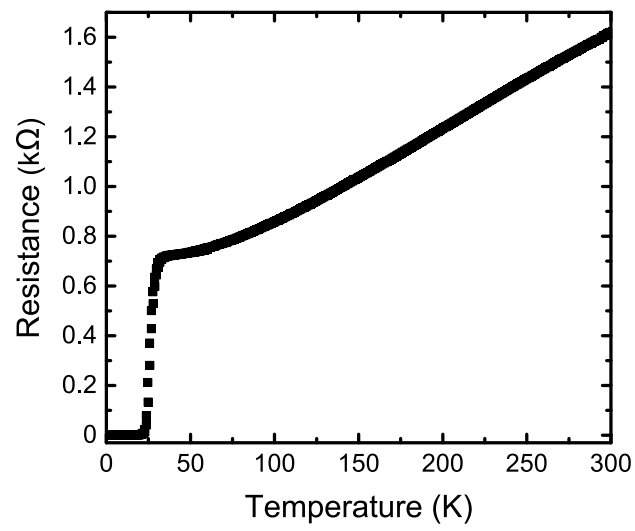

(b)

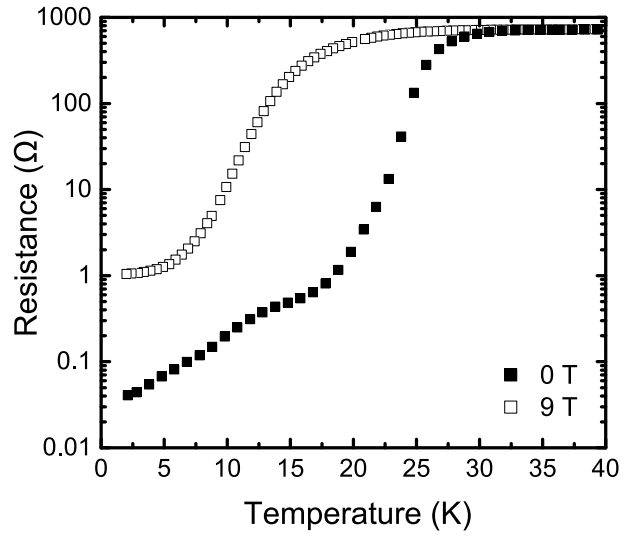

Figure 6.6: (a) Resistance versus temperature measurement on the superconducting short of the MottSQUID ring. (b) Low temperature close-up showing the restance in zero field (solid symbols) and in a $9 \mathrm{~T}$ applied field (open symbols).

further in a step-wise manner. In a $9 \mathrm{~T}$ field, the $1 \Omega$ resistance is recovered for the lowest temperatures. This suggests that the step-wise decrease is part of a broad superconducting transition into a weakly superconducting state. The LSCO leads remain superconducting in the $9 \mathrm{~T}$ field at $2 \mathrm{~K}$; in other experiments we have also seen that the critical field for LSCO is higher than $9 \mathrm{~T}$ at $2 \mathrm{~K}$, see e.g. chapter 5. From this we can already conclude that this structure does not function as the superconducting short needed to complete the MottSQUID rings. The structure was expected to function on two effects, a broad $c$-axis contact to each layer and ramp-edge contacts on the sides. The absence of a superconducting short or even a Josephson effect is attributed to the many processing steps, combined with the short coherence length of LSCO $[196,197]$. It is not clear from the measurement if the resistive contact is formed to the first or second layer or to both of the layers. The most likely scenario is that resistive contact is only formed between the first and third layer. The top of the second layer is not etched during any of the processing steps, leaving it untouched and clean for the third layer to form a superconducting contact in the $c$-axis direction. The first layer is etched into during the structuring of the second layer and the ramp area was etched upon directly. As the coherence length is about an order of magnitude smaller in the $c$-axis direction [194], the contact would rely on the two ramp-edge junctions. Of these two junctions, the junction between the second and the third layer is similar in design to the LSCO/LSCO ramp-edge junctions described in chapter 3, where a Josephson contact is formed. This leaves only the ramp-edge junction between the first and the third layer as the origin for the remaining resistance. It is also this resistance that obscures any Josephson nature in the other junction in transport measurements.

Alternative designs to avoid this construction of the superconducting short are 
(a)

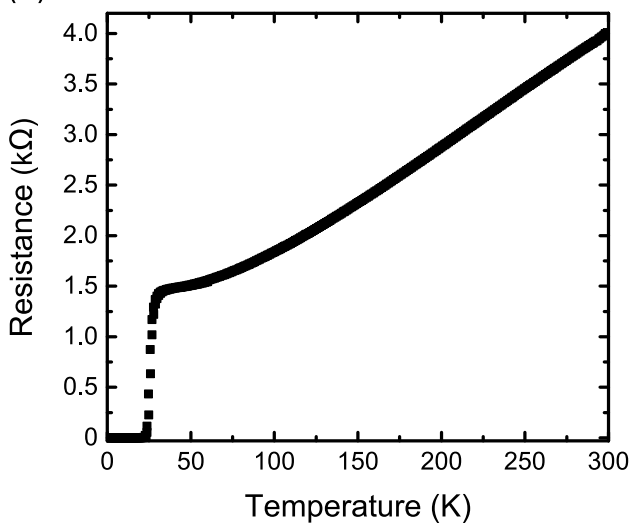

(b)

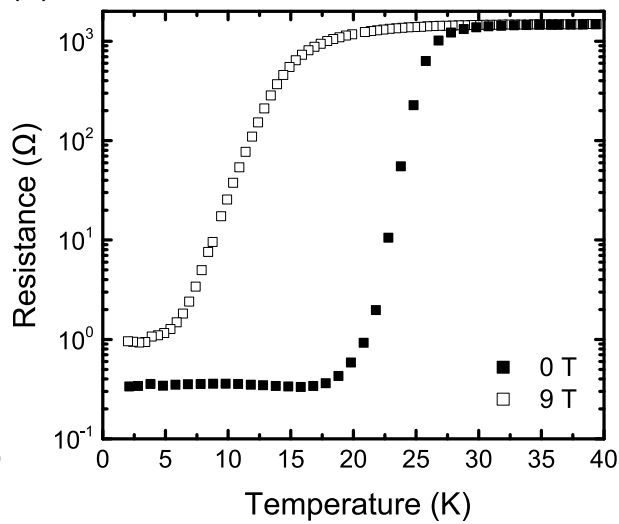

Figure 6.7: (a) Resistance versus temperature for a completed MottSQUID ring with two contact leads, measuring the type-II Bose-Mott insulator junction and the superconducting short in parallel. (b) Low temperature close-up of the resistance in zero field (closed symbols) and in a $9 \mathrm{~T}$ applied field (open symbols).

described in more detail in $\S 6.6$. The design considerations focus on on minimizing damage on the ramps by defining the ramps as a final step before the deposition of the third layer and to have the ramps face the same direction, to avoid etching directly on the ramp. Another possibility would be to replace the third LSCO layer with a metallic superconductor like niobium. This will somewhat relax the requirements for the interface between the materials due to the longer coherence length of niobium, and it removes a high temperature processing step. However, it does introduce further complications regarding niobium oxide interlayers that would require, for example, extra gold interlayers. Initial experiments with niobium on one of the MottSQUID were unsuccessful, probably due to the already present damage to the ramps, sample degradation or the formation of a niobium oxide interlayer (effectively changing the doping level of the LSCO by oxygen depletion).

The absence of a superconducting short is also confirmed by a measurement of one of the central MottSQUID structures of figure 6.3; a resistance versus temperature measurement on this structure is shown in figure 6.7. Here, a finite, temperature independent resistance of around $0.3 \Omega$ remains below $T_{c}$. The gradual decrease of the resistance that was seen for the MottSQUID short test structure is not observed. The shorted part of the MottSQUID junction is two times wider than the test structure and the $c$-axis contact area is also about two times larger, which could explain the more uniform nature of the remaining resistance.

Since we have established that there is no superconducting short in the MottSQUID rings, the next section will focus on the isolated junction structures instead, the second structure in figure $6.3(\mathrm{~b})$. 
(a)

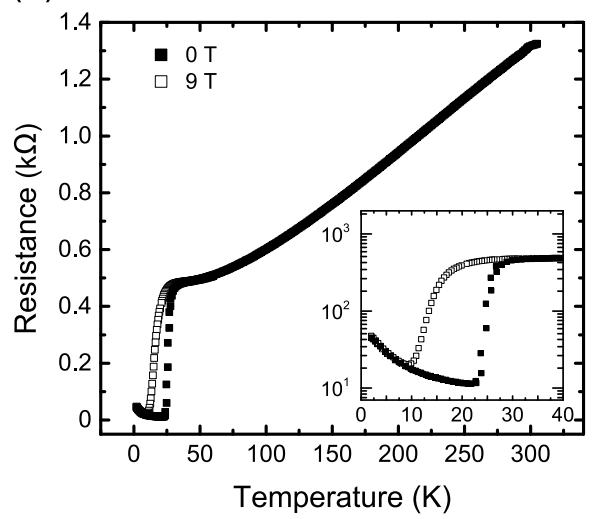

(b)

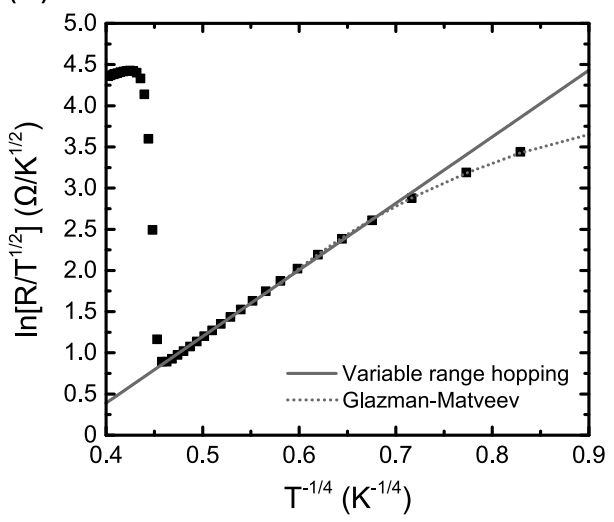

Figure 6.8: Resistance versus temperature for a candidate type-II BoseMott insulator ramp-edge junction. (a) Measurement in zero field and 9 $\mathrm{T}$, the inset shows the finite increasing resistances below $T_{c}$ of the LSCO leads. (b) The resistance at low temperature is dominated by variable range hopping, shown by the linear dependence of $\ln \left[R T^{-1 / 2}\right]$ vs. $T^{-1 / 4}$ (solid line). At the lowest temperatures there is a cross-over to a $T^{4 / 3}$ temperature dependence (dotted line), indicative of inelastic tunneling described by the Glazman-Matveev theory [131].

\subsection{Experimental results}

Figure 6.8 shows resistance versus temperature for a $10 \mu \mathrm{m}$ wide $\mathrm{La}_{1.85} \mathrm{Sr}_{0.15} \mathrm{CuO}_{4^{-}}$ $\mathrm{La}_{1.95} \mathrm{Sr}_{0.05} \mathrm{CuO}_{4}-\mathrm{La}_{1.85} \mathrm{Sr}_{0.15} \mathrm{CuO}_{4}$ junction. Below the $T_{c}$ of the two LSCO leads an upturn of the resistance is observed. This upturn is independent of the applied magnetic field as can be seen for the curve measured at $9 \mathrm{~T}$. The temperature dependence of the upturn follows that of variable range hopping $(\mathrm{VRH})$, which has a $T^{-1 / 4}$ temperature dependence. In figure $6.8(\mathrm{~b})$ we plot $\ln \left[R T^{-1 / 2}\right]$ vs. $T^{-1 / 4}$, the solid line, following the equation for variable range hopping (Mott's law):

$$
R=R_{0} T^{2 w} \mathrm{e}^{\left(T_{M} / T\right)^{w}}
$$

where $R$ is the resistance, $T_{M}$ is the Mott temperature and $w$ is a factor ranging from $1 / 2$ to $1 / 4$, depending on the influence of Coulomb interaction on the density of states at the Fermi level. This particular dependence with $w$ close to the $1 / 4$ value of pure VRH has been observed in the underdoped, spin-glass phase of $\mathrm{La}_{2-\mathrm{x}} \mathrm{Sr}_{\mathrm{x}} \mathrm{CuO}_{4}$ [198]. At the lowest temperatures the resistance deviates from the VRH dependence towards a dependence that can be described by a $T^{4 / 3}$ dependence (dotted line). This dependence is characteristic for inelastic tunneling through a limited number of impurities, with this particular dependence dominated by two-impurity tunneling [131].

The tunneling nature of the contact also appears in the $I V$ characteristics. Figure 
6.9(a) shows numerically derived $d I / d V$ measurements for different temperatures. An example of the $I V$ characteristics can be found in appendix A, figure A.1(c). Below $18 \mathrm{~K}$ a SIS or NIS tunneling spectrum is observed in the $d I / d V$-characteristics. The spectrum is characterized by broad coherence peaks, indicative of inelastic tunneling [130]. Such a tunneling spectrum can be fitted by a modified BlonderTinkham-Klapwijk (BTK) model [199], by incorporating a spectral broadening term in the Bogoliubov equations $[129,130]$. The relatively low resistance of the tunneling contact limits measurement of the coherence peaks to the smallest junctions $(10-15 \mu \mathrm{m})$; for larger junctions, the current required to reach the gap voltage exceeds the critical current of the LSCO leads. Figure 6.9(c) shows the superconducting gap extracted from the coherence peaks of figure 6.9 (a) versus temperature. The gray line is a least squares fit with the BCS gap equation for $5-16 \mathrm{~K}$. It gives a $T_{c}$ of $16.9 \mathrm{~K}$ and a combined gap of $\sim 9.4 \mathrm{meV}$ for the tunneling contact. Figure $6.9(\mathrm{~b})$ shows the behavior of the tunneling contact in a magnetic field. As was also seen in figure 6.8(a), the zero bias conductance is virtually unaffected by the magnetic field, showing only a slightly smaller conductance for the highest applied fields. Both the height of the coherence peaks and the superconducting gap decrease with field. Figure 6.9(d) shows the size of the gap versus field, which is observed to decrease linearly with increasing field (gray line).

The junction geometry allows for several different scenarios that can lead to a tunneling spectrum as seen in figure 6.9(a). The junction can be seen as two back to back NS contacts, one between $\mathrm{La}_{1.85} \mathrm{Sr}_{0.15} \mathrm{CuO}_{4}$ and $\mathrm{La}_{1.95} \mathrm{Sr}_{0.05} \mathrm{CuO}_{4}$ at the ramp interface, and the other between $\mathrm{La}_{1.95} \mathrm{Sr}_{0.05} \mathrm{CuO}_{4}$ and $\mathrm{La}_{1.85} \mathrm{Sr}_{0.15} \mathrm{CuO}_{4}$ in the top electrode. The $\mathrm{La}_{1.95} \mathrm{Sr}_{0.05} \mathrm{CuO}_{4}$ layer is $50 \mathrm{~nm}$ thick, but it is expected that at least part of it is superconducting due to a proximity effect from either of the two $\mathrm{La}_{1.85} \mathrm{Sr}_{0.15} \mathrm{CuO}_{4}$ electrodes.

Using the Mott temperature from equation 6.4, we can estimate the average hopping length $\bar{r}$ via

$$
\bar{r}=a_{B}\left(\frac{T_{M}}{T}\right)^{1 / 4},
$$

where the Bohr radius $a_{B}=4 \pi \epsilon \epsilon_{0} \hbar^{2} / m^{*} e^{2}$, with $\epsilon \epsilon_{0}$ the perimittivity and $m^{*}$ the effective mass [200]. We find $T_{M} \approx 4300 \mathrm{~K}$ from the fit in figure 6.8 , comparable to values found in literature [198]. When taking $\epsilon=20-40$ as typical values for cuprates, this results in an average hopping length of $7-15 \mathrm{~nm}$ at the lowest temperatures. Therefore, hopping takes place via a limited number of impurities below $\sim 4 \mathrm{~K}$. This is also the regime where the crossover to the GlazmanMatveev behavior is observed, indicating that two impurity scattering starts to dominate.

From this we conclude that we are dealing with an SINS contact, where the tunnel barrier is most likely located at the ramp interface. The NS contact to the top electrode is expected to be very transparent, since it is created in situ. This will only give a small contribution to the total conductance curve and it is negligible 
(a)

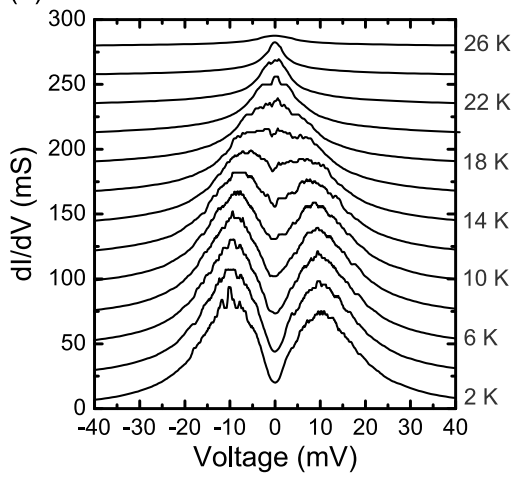

(b)

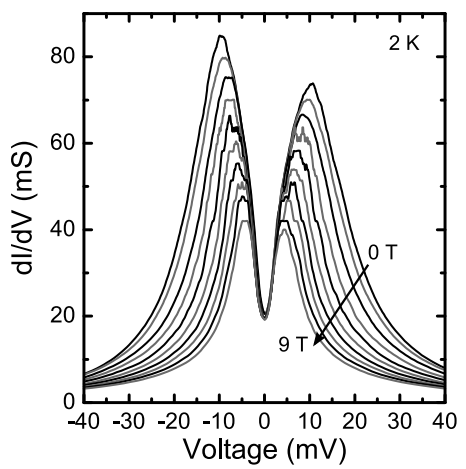

(c)
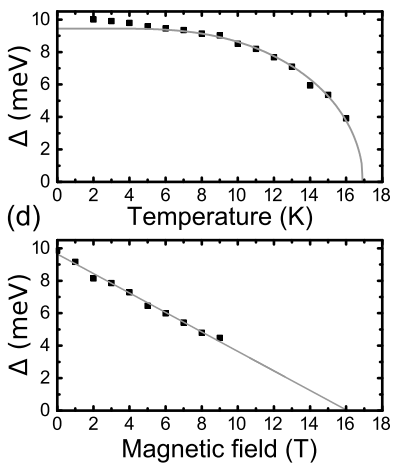

Figure 6.9: dI/dV measurements as function of temperature and field for a candidate type-II Bose-Mott insulator junction (10 $\mu \mathrm{m}$ wide). (a) Temperature dependence of the $\mathrm{dI} / \mathrm{dV}$ characteristics from $2 \mathrm{~K}$ to $26 \mathrm{~K}$. All curves, expect the curve at $2 \mathrm{~K}$ are offset for clarity. (b) $\mathrm{dI} / \mathrm{dV}$ at $2 \mathrm{~K}$ in a perpendicular magnetic field varying from 0 to $9 \mathrm{~T}$ in steps of $1 \mathrm{~K}$. (c) Superconducting gap versus temperature extracted from the peak positions in (a). The gray curve is a least squares fit using the BCS gap equation for the temperature range $5-16 \mathrm{~K}$ with $\Delta_{0}=9.4 \mathrm{meV}$ and $T_{c}=16.9 \mathrm{~K}$. (d) Superconducting gap versus magnetic field extracted from the peak positions in (b). The gray line is a linear least squares fit to the data points.

compared to the signal from the bottom interface. The inelastic tunneling nature of the barrier is confirmed by both the temperature dependence and the spectral broadening of the coherence peaks in the $d I / d V$ measurements. The temperature dependence is mostly determined by the $\mathrm{VRH}$ in the $\mathrm{La}_{1.95} \mathrm{Sr}_{0.05} \mathrm{CuO}_{4}$ layer. At the lowest temperatures this crosses over into the Glazman-Matveev regime, where this inelastic tunneling process also results in an additional non-linear current-voltage characteristic. This makes it difficult to accurately fit the $d I / d V$ characteristics of figure 6.9 , since there are too many fitting parameters.

Due to the additional resistance of the interlayer, which is non-linear and temperature dependent, the $d I / d V$ characteristic is difficult to fit with a simple model. Using the modified BTK theory with spectral broadening, we can get an estimate of the barrier parameters when we assume the additional resistance is not voltage dependent. In doing so, we can estimate the barrier parameter $Z$ to be smaller than 1 and the spectral broadening to be at most $40 \%$ of the superconducting gap of the $\mathrm{La}_{1.85} \mathrm{Sr}_{0.15} \mathrm{CuO}_{4}$. This barrier parameter is reasonable, since we have seen in chapter 3 that a barrier with a low normal state resistance is also found in $\mathrm{La}_{1.85} \mathrm{Sr}_{0.15} \mathrm{CuO}_{4}-\mathrm{La}_{1.85} \mathrm{Sr}_{0.15} \mathrm{CuO}_{4}$ ramp-edge Josephson junctions.

The properties of the junctions have also been measured under RF irradiation using a small looped antenna placed near the sample in the cryostat. The general trend is a suppression of the critical current of the superconducting leads for high applied power which is a strong function of the RF frequency. We attribute this to a local heating effect, which we have also observed for the Shapiro step measurements 


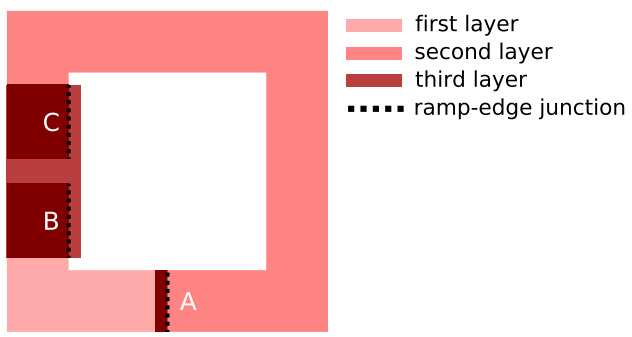

Figure 6.10: Proposal for a new sample design to measure Mott vortices in a ring structure with a superconductor/type-II Bose-Mott insulator/superconductor junction (A). The ramps in layer 1 and 2 (B and $\mathrm{C}$ ) are created by ion milling in the same sample-beam geometry to avoid excess damage to the ramp surface.

on the NCCO/NCCO and LSCO/LSCO Josephson junctions discussed in chapter 3. The heating effect observed by RF irradiation is assumed to originate from standing waves induced in parts of the sample. These standing waves can occur in the devices, the buffer layer or the substrate itself. The RF frequency was varied between 6 and $18 \mathrm{GHz}$, which corresponds to a wavelength in vacuum varying from 50 to $16 \mathrm{~mm}$. The heating effect is a function of both the efficiency of the energy transfer and the wavelength of the radiation. We see strong effects near the optimal frequencies of the RF loops, where the circumference of the loop is close to $\lambda / 2$; there are two loops, one has an optimal frequency around $15 \mathrm{GHz}$ and the other around $6.5 \mathrm{GHz}$. LSAT has low dielectric loss and a relative permittivity of around 23 in the $\mathrm{GHz}$ range [201], yielding a refractive index of $\sim 4.7$. The effective $\lambda / 2$ in LSAT is therefore $2 \mathrm{~mm}$ and $5 \mathrm{~mm}$ for the two loops. These correspond to the typical device lengths and the size of the substrate, respectively. The heating effects are therefore most likely caused by standing waves induced in the substrate.

\subsection{Discussion}

We have demonstrated that it is possible to fabricate devices suitable for an experiment looking for Mott vortices in a candidate type-II Bose-Mott insulator. However, some experimental challenges remain, namely to improve the current range available and the fabrication of a good superconducting short for the MottSQUID ring structures. For the design in figure 6.3, the current range is limited to $1-2 I_{0}$ before the critical current of the measurement leads is reached. In this section we will discuss several options for future experiments that can address these issues.

The first option is an improved version of the ring design. In the current design it was found that the third superconducting layer does not make a good superconducting contact with the underlying two layers. We attribute this to damage 
on the first ramp during the etching of the ramp in the second set of layers. This was also seen in the TEM cross sections of the $p n$-junctions discussed in chapter 3 . Here, the ramp that was defined on the side of the mesa that was directly in the ion beam showed many dislocations and ion damage. This can be avoided by defining all the ramps in the sample in the same sample-beam geometry. Figure 6.10 shows the proposed design where the main contact of the third layer with the first and second layer is made along the inside of the ring structure (junctions B and $\mathrm{C}$ in the figure).

A second, related improvement would be to increase the layer thickness and the width of the superconducting layers to increase the critical current. This holds for both the ring structures and for the individual junctions. With the increased critical current, a larger range of currents can be explored in looking for the Mott vortex current quanta in the type-II Bose-Mott insulator before superconductivity is lost in the connecting layers.

As was seen in the experiments in chapter 3 , a $\mathrm{La}_{1.85} \mathrm{Sr}_{0.15} \mathrm{CuO}_{4}-\mathrm{La}_{1.85} \mathrm{Sr}_{0.15} \mathrm{CuO}_{4}$ ramp-edge contact has a significantly lower critical current than a $\mathrm{La}_{1.85} \mathrm{Sr}_{0.15} \mathrm{CuO}_{4}$ strip line. This will alway remain a limiting factor, especially in the design of the ring structures, as here the size is limited by the size of the flip-chip measurement configuration. An alternative method for defining the device might be found in the use of ionic-liquid gating in a electric double layer transistor (EDLT) geometry. Ionic liquid gating has recently proven to be a powerful tool in the gating of oxide materials. Especially for cuprate superconductors, conventional gating cannot provide the large electric fields necessary to change the carrier density due to the high intrinsic carrier density and large screening. With ionic liquid gating, charges are brought as close to the interface as the size of a single molecule, which results in large electric fields in the top layer of the material that is being gated. This technique has already been shown to be able to fully tune the superconductivity in materials like $\mathrm{La}_{2-\mathrm{x}} \mathrm{Sr}_{\mathrm{x}} \mathrm{CuO}_{4}$ [202] and $\mathrm{YBa}_{2} \mathrm{Cu}_{3} \mathrm{O}_{7-\mathrm{x}}$ [203]. For our experiment, ionic liquid gating can be used in two ways: to selectively deplete a small section in a superconducting ring or to tune an underdoped ring into superconductivity, except for a small section. Both have their individual merits. The first technique would be able to continuously tune a small section of the ring through the region where the type-II Bose-Mott insulator phase is predicted to exist. This does however require nanometer precision and uniformity for the area that is to be gated. The second technique has similar restrictions on the lateral resolution of the gating, but, since the screening will be lower in the underdoped region, we can expect some bleeding of the gating effect underneath the shielded areas, making it possible to make the shielded area larger, facilitating fabrication. The potential advantage of ionic liquid gating is twofold: (1) it should create the cleanest possible contact between the superconductor and the type-II Bose-Mott insulator, as no processing is required to create the junction; and (2) especially in the first scenario, a single device can be used to map out the type-II Bose-Mott insulator phase diagram by tuning the carrier density from optimal doping towards zero doping. Using this technique does require wide junctions to reach the regime of being able to measure several current quanta, due to the unit cell thickness of the films. 
(a)

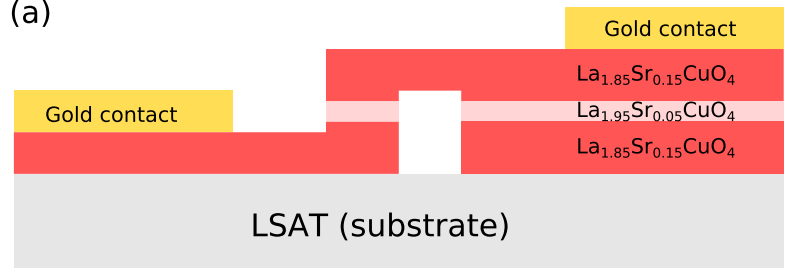

(b)

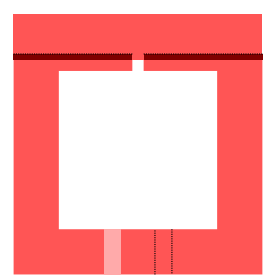

Figure 6.11: Proposal for a new sample design to measure Mott vortices in a perpendicular configuration. (a) Side view of the junction area, the structure is fabricated using argon ion milling to define the outline of the mesa structure and expose the bottom layer. Focused ion beam etching is used to define the final superconductor/type-II Bose-Mott insulator/superconductor stack. (b) Top view where the junction from (a) is used in a ring structure closed with two ramp-edge contacts using a second superconducting layer

A final possibility would be to replicate the structure used by Bozovic et al. [194], i.e. fabricate a junction in the $c$-axis direction. This can either be done in a mesa structure as described in chapter 5 , where only a direct transport measurement is possible, or a hybrid $c$-axis/ring structure can be designed as shown in figure 6.11(a). Here, the junction is fabricated by shaping a zig-zag structure with focused ion beam (FIB) etching, which has already been successfully employed for intrinsic $c$-axis LSCO Josephson junctions [204]. This structure can either be used as a single junction or used as part of a ring structure, where the ring is closed by a second superconducting layer via two ramp-edge contacts, see figure 6.11(b). This second layer will preferably be deposited before the FIB structuring, to avoid damage and contamination near the junction. The advantage of this structure is that there is no processing done on the actual junction interface, it is all grown in one go.

Following the considerations in the theory section of this chapter, the electrodes do not necessarily have to be superconducting. Therefore, another $c$-axis contact possibility would be a junction without superconducting layers, i.e. a $\mathrm{La}_{1.95} \mathrm{Sr}_{0.05} \mathrm{CuO}_{4}$ layer sandwiched between overdoped $\mathrm{La}_{2-\mathrm{x}} \mathrm{Sr}_{\mathrm{x}} \mathrm{CuO}_{4}$ layers. Due to the large contact area, sufficient current can be applied to reach the level of the predicted current quantum. The addition of the overdoped layers eliminates any Schottky barriers at the interface and a four probe measurement across the layer stack would therefore only measure the properties of the $\mathrm{La}_{1.95} \mathrm{Sr}_{0.05} \mathrm{CuO}_{4}$ interlayer. It is also expected that the overdoped $\mathrm{La}_{2-\mathrm{x}} \mathrm{Sr}_{\mathrm{x}} \mathrm{CuO}_{4}$ layers will result in a lower contact resistance to e.g. gold electrodes, due to the higher intrinsic carrier density, creating a weaker Schottky barrier. If it turns out that the nature of the Mott vortices in $\mathrm{La}_{2-\mathrm{x}} \mathrm{Sr}_{\mathrm{x}} \mathrm{CuO}_{4}$ is more two-dimensional than three-dimensional with the formation of Mott "pancakes" [185], a $c$-axis configuration would be preferred over an in-plane contact to probe the formation of these Mott pancakes as they are expected to couple to each other in the $c$-axis direction. The VRH transport we have observed in the $\mathrm{La}_{1.95} \mathrm{Sr}_{0.05} \mathrm{CuO}_{4}$ can provide a natural shunt resistance, allowing for a current to be applied, and since both layers are not superconducting 
(apart for perhaps an interface layer [205]), there is no Josephson effect that can obscure effects due to the current quantization.

\subsection{Conclusions}

In this chapter we have shown that we have made the first steps towards an experiment to search for Mott vortices in a candidate type-II Bose-Mott insulator. We show that we can combine ramp-edge technology and LSCO in three different deposition steps, where all three layers are superconducting. Performance of the final layer and in particular the superconducting short still require further experiments. We have successfully sandwiched a candidate type-II Bose-Mott insulator between two superconductors and recover the known properties of this layer in our experiments: the $\mathrm{La}_{1.95} \mathrm{Sr}_{0.05} \mathrm{CuO}_{4}$ layer shows variable range hopping in transport, what is expected for the spin glass phase at low temperatures. The remaining issues in the experiment are the available current and the completion of a superconducting ring. With the current dimensions, the leads can only carry enough current for 1 to 2 current quanta and the ring is not yet fully superconducting. We propose several design modifications and new geometries to address these issues. Furthermore, we suggest alternative experiments for exploring a $c$-axis geometry and we suggest using ionic liquids to define the junctions electrostatically to achieve cleaner interfaces.

\section{Appendices}

\section{A Current source limitations}

Figures A.1(a) and (c) compare $I V$ measurements in the bath cryostat (a) to measurements done in the PPMS (c). Here, the difference between the current sources becomes evident around the critical current of the superconducting LSCO leads. The PPMS is operated in a constant current mode that adjusts its output to match the impedance of the load. The current source used in the bath cryostat is designed to have a $50 \Omega$ load. This results in a significant drop of the output voltage when the measured devices revert to the resistive state, due to the increased load. This can be seen in figure A.1(b). Here, the voltage across a $1 \mathrm{k} \Omega$ resistance is recorded during the measurement of one of the junctions. At low bias the voltage follows the programmed sawtooth shape; when the critical current of the LSCO leads is reached, the changing load causes a sharp dip in the recorded voltage. This dip creates artifacts in the final $I V$ characteristic, creating an overshoot before the curve jumps to the normal state voltage. The combination of the sudden load change, the (dis)charging time of the capacitors in the low-pass filters and a heating effect can explain the overshoot and the transition to the normal state at a lower current. This theory is confirmed by a measurement in a flow cryostat, where the sample is cooled by helium gas. Here, the cooling power is lower, result 
in a more dramatic effect when the critical current is reached. Figure A.1(d) shows the corresponding $I V$ characteristic, where the overshoot of figure A.1(c) has expanded into something that looks like a negative resistance, reminiscent of a Gunn diode or an Esaki diode [80].

From this we can conclude that measurements using the function generator around large impedance changes are not reliable. When we compare the measurement to the curves measured in the PPMS, we see that the measurement is reliable for low bias and for high bias, sufficiently larger than the critical current of the LSCO lead. In the low bias regime in particular, the measurement is more accurate than the PPMS measurement, due to the higher resolution in the voltage measurements.

(a)

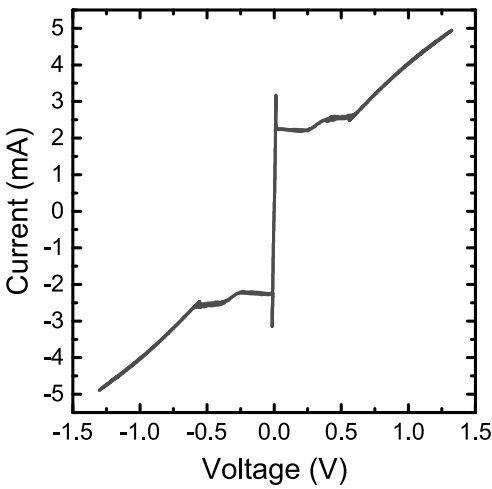

(c)

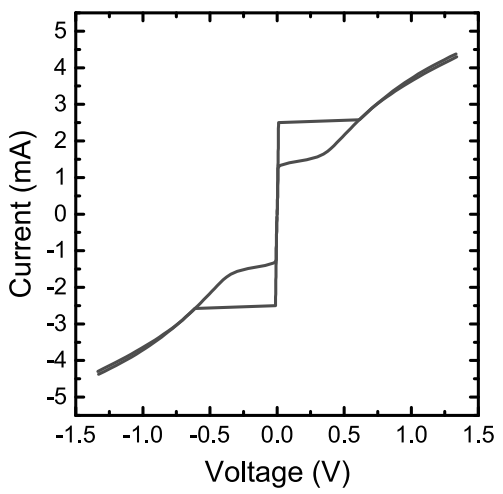

(b)

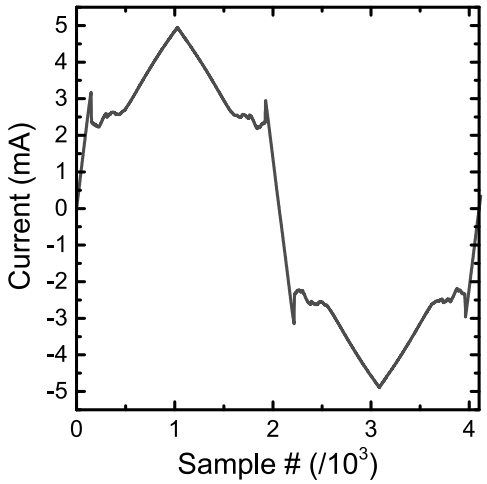

(d)

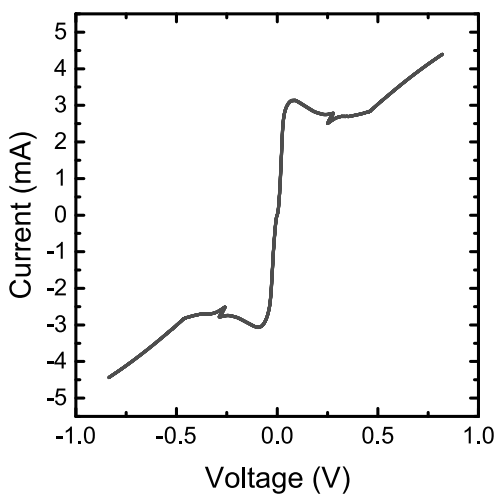

Figure A.1: Effect of current source limitations on junction $I V$ characteristics. (a) $I V$ measurement at $4.2 \mathrm{~K}$ for a $20 \mu \mathrm{m}$ junction measured in the bath cryostat using custom electronics. (b) Trace of the measured current during one sawtooth period. (c) $I V$ measurement at $4 \mathrm{~K}$ on the same junction using the PPMS electronics, the hysteresis is caused by resistive heating. (d) $I V$ measurement on a similar junction on a different chip measured in a flow cryostat at $5 \mathrm{~K}$ showing the effects of excess heating. 



\section{Epilogue}

In this thesis, the successful combination of electron-doped $\mathrm{Nd}_{1.85} \mathrm{Ce}_{0.15} \mathrm{CuO}_{4}$ (NCCO) and hole-doped $\mathrm{La}_{1.85} \mathrm{Sr}_{0.15} \mathrm{CuO}_{4}$ (LSCO) is demonstrated, where both materials are superconducting. The combination is explored in two geometries, a $c$-axis junction and an in-plane ramp-edge junction. In both cases, an insulating barrier is found at the interface between NCCO and LSCO. For the ramp-edge junctions, the barrier has been characterized and it appears to be formed by a combination of an electronic depletion and a strain depletion effect. The experiments cannot confirm that the electronic depletion layer has a Mott insulator character expected from theory $[10,12]$. Further experiments with the layer order reversed or with electrodes of different doping level are required to determine the exact nature of the barrier.

This leads us to the question whether this material system is suitable for exploring potential excitonic effects in Mott insulators further. From the HAADF-STEM cross-section of the LNL device (LSAT-NCCO-LSCO), we can conclude that this configuration is not suitable for a bilayer exciton experiment due to the intrinsic roughness of the NCCO layer. Exciton formation is expected to occur between two adjacent $\mathrm{CuO}_{2}$ planes or $\mathrm{CuO}_{2}$ planes separated by a dielectric layer $[13,25]$. This requires a sharp interface. The LLN configuration (LSAT-LSCO-NCCO) might be a better alternative, because the LSCO layer is very smooth, occasionally showing the substrate structure even for $150 \mathrm{~nm}$ thick films. LSCO has been shown to grown in a layer-by-layer fashion [167] and can therefore be used to create an atomically flat bottom electrode. The downside of the LLN configuration is the unfavorable temperature profile that is used during deposition, where the LSCO layer is heated above its deposition temperature for deposition of the NCCO layer.

When combining NCCO and LSCO it appears that the cleanest interface is obtained in a ramp-edge geometry and not in a bilayer geometry, as evidenced by HAADF-STEM measurements. This is combined with the added benefit of relaxed annealing conditions for the NCCO layer. An experiment can be devised where only the interface between two materials along the ramp is important. Consider a bottom electrode of the undoped parent compound $\mathrm{Nd}_{2} \mathrm{CuO}_{4}$ with a ramp etched into it. This layer can be rather thick to get a long ramp. On top of this we can grow thin layers of selected electron and hole-doped cuprates. From the experiments on LSCO/NCCO ramp-edge junctions discussed in this thesis, we know that a barrier forms naturally at the interface between LSCO and NCCO on the ramp, through a combination of strain and electronic effects. This would also create a barrier in the proposed experiment, resulting in the sought after geometry for the exciton experiments, which require thin, oppositely doped layers in close proximity. With a ramp that is sufficiently long, the new in-plane bilayer can be 
extracted by etching away all the material at the bottom of the ramp. Contacts can be made to both layers by standard photolithography if the size of the device is big enough or by electron beam lithography if more control is required. Another way to measure the interface would be to use a local probe like scanning tunneling microscopy or conducting atomic force microscopy. An example of a signature to look for in a local probe experiment is enhanced tunneling when excitons form and condense following the experiments of Eisenstein et al. [19].

Moreover, a structure like this is very suitable to realize a theoretical prediction of quantization of the magnetization of a bilayer exciton ring as function of the flux between the layers [206]. The ramp-edge structure presents a natural way for the flux to penetrate the bilayer structure for a perpendicular applied field due to the bilayer being tilted at an angle on the ramp.

Another option is to exploit electric double layer transistor (EDLT) technology using ionic liquids to focus on just LSCO and tune a lightly doped LSCO film partly towards electron doping [202]. Experiments with Ce doping in the Tphase $\mathrm{La}_{2} \mathrm{CuO}_{4}$ have shown that electron doping is possible in this phase, but that the films only become more insulating [65]. This does not have to limit our proposed experiment and the EDLT charge doping might have a different effect than Ce doping. Since EDLT on LSCO only acts on a single unit cell layer [202], an experiment on two unit cells of LSCO would already yield the sought after structure for the bilayer exciton experiments.

A different route towards oppositely doped bilayers in strongly correlated electron systems can potentially be found in the rapidly developing field of two-dimensional electron gases (2DEGs) in oxide systems we alluded to in the introduction of this thesis. Here, the parallel to the exciton condensation experiments in semiconductor systems is even stronger. The work-horse 2DEG is found at the STO/LAO interface. There is even evidence for mixed carrier sign transport with indirect evidence for a parallel electron-hole bilayer when the LAO layer is capped with STO [207, 208]. For the LAO/STO system, both layers are band insulators, but there is also theoretical work and experimental evidence that shows that a 2DEG can also form when STO is combined with Mott insulators like $\mathrm{LaVO}_{3}$ [209], $\mathrm{LaTiO}_{3}$ [210], $\mathrm{GdTiO}_{3}$ [211] and $\mathrm{YTiO}_{3}$ [212]. The question remains how much of the Mott insulator character is reflected in the 2DEG, since it is mostly found inside the STO. It is interesting to note that $\mathrm{GdTiO}_{3}$ shows hole-like carriers, so this might intrinsically give a close proximity of electrons and holes.

Here, measurements similar to those done on semiconductor systems [20,171,213, 214] can be performed by using a combination of back gates and top gates. For LAO/STO it has been shown that the 2DEG can be manipulated using both back gating [215] and top gating [216]. This can be applied to the structures of Huijben et al. [208] to attempt independent contact. A first experiment would be to contact both layers and use top gating to pinch off the hole-doped top layer or back gating to pinch off the electron-doped bottom layer [171]. In this experiment the evolution from mixed transport to fully electron(hole)-like transport can be investigated using Hall measurements. The experiment can also be a probe for the layer separation. A four point measurement should show only ohmic behavior for 
(a)

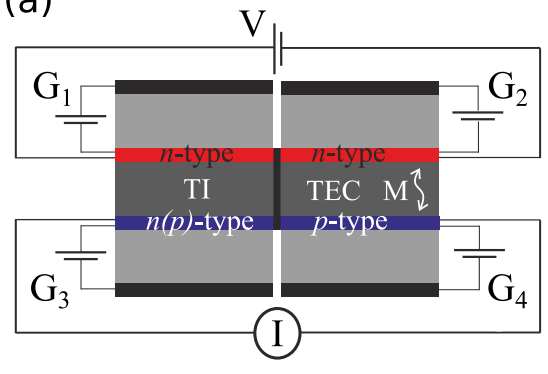

(b)

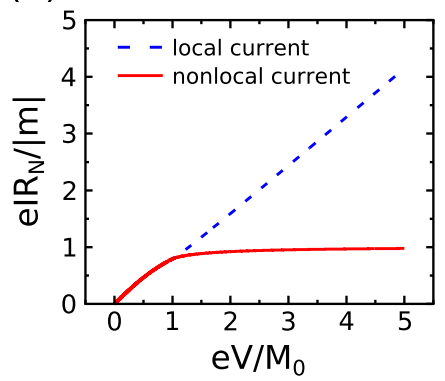

Figure E.1: (a) Schematic device architecture for probing exciton condensation in topological insulators (TI). Individual gates $\left(\mathrm{G}_{1}\right.$ to $\left.\mathrm{G}_{4}\right)$ can tune the topological surface states between $n$ and $p$-type character. The $n p$ configuration on the right side results in a Coulomb attraction $M$ that leads to a topological exciton condensate (TEC) [217]. The two electrodes on the left can be tuned individually to probe the TEC. (b) A current through the top layer of the device in (a), results in an entangled, nonlocal current below the exciton gap in the bottom surface via Andreev reflection on the TEC. $M_{0}$ denotes the amplitude of the Coulomb interaction, $m$ is the effective mass and $R_{N}$ is the normal-state resistance.

the electron doped layer when the hole doped layer is pinched off. Any (tunneling) transport from the electron layer into the hole layer should result in non-linear $I V$ characteristics. If the layers are truly separate, a soft contact to only the top layer can also be attempted by using a soft deposition technique for the electrodes like thermal evaporation or electron-beam evaporation [216].

Finally, we would like to broaden our discussion of exciton physics in strongly correlated systems to unconventional systems. In parallel to the work presented in this thesis, we have also worked on topological insulators ${ }^{\dagger}$ (TI) [220-222]. It was not related to the central topic of this thesis, but we would like to highlight one theoretical prediction that touches upon the search for exciton physics in unconventional systems. Bilayer exciton formation and condensation is predicted to occur for thin topological insulators where the two opposite surfaces are tuned to electron and hole-like states with electrical gates, in a geometry like the right side of figure E.1(a) [217]. We have used knowledge of superconducting Josephson junctions to theoretically study the transport properties of a topological exciton condensate (TEC) [222]. We have investigated two geometries: the analogue of a normal metal - superconductor (NS) contact, the TI-TEC contact, and the analogue of a SNS contact, TEC-TI-TEC.

\footnotetext{
$\dagger$ Topological insulators are a recently discovered class of materials that are characterized by an insulating bulk and conducting surface states. These surface states appear as a single Dirac cone in momentum space and are robust against non-magnetic disorder. The surface states have a spin-momentum coupling that circles the Dirac cone, dictated by strong spin-orbit coupling, which is the driving force behind the formation of the topological insulator state. A good introduction into topological insulators can be found in refs. [218] and [219].
} 
In the first configuration, we find that Andreev reflection on the TEC is a nonlocal process, where an incoming electron gets reflected as a hole in at the other surface of the TI. Below the exciton gap, this results in a fully entangled current, since all other processes at the TI-TEC interface, other than direct reflection, are suppressed, see figure E.1(b). Furthermore, by tuning the left electrodes of figure E.1(a) from a $n n$ arrangement to a $n p$ arrangement, the Andreev reflection switching from specular reflection to retro reflection.

In the TEC-TI-TEC configuration, we find a bound state that shows a gapless dispersion for perpendicular incidence. These bound states are not protected by parity like bound states in a topological superconductor [223] due to a degeneracy in layer. However, in a non-equilibrium state, the system can show a crossover from a current phase relationship that is $2 \pi$ periodic to a $4 \pi$ periodicity, accompanied by fractional charge transport, provided an ac measurement can be performed faster than relaxation processes.

Now, we would like to discuss briefly how the proposed TI-TEC and TEC-TI-TEC configurations can be realized in practice. In graphene experiments, there is a promising development using sacrificial resist layers to realize suspended graphene and suspended top gates $[224,225]$. Combined with selective bottom gating this has already been used to create in-plane $p n$-junctions [225]. An extension of these experiments to topological insulators would already come close to the desired architecture of figure E.1(a). The main remaining challenge is to realize separate contacts to the top and bottom surface of the structure, and to prevent cross-talk via the sides of the flake.

In conclusion, we have discussed how cuprates can be used to bring electron and hole-like layers in close proximity as a first step towards exploring exciton physics in doped Mott insulators. Furthermore, we have suggested different measurement geometries and material systems that can also be suitable to realize closely space oppositely doped bilayers in strongly correlated systems. Finally, we have briefly described a theoretical proposal to study exciton condensation in an unusual system, a thin topological insulator. We predict nonlocal Andreev reflection and fractional charge transport, and we have discussed how this proposal can be realized in an experiment. 


\section{Summary}

There are many parallels between semiconductors and high-temperature superconducting cuprates, both from a historical and a physical perspective; both are characterized by a gapped insulating ground state and become conducting upon doping. The origin of the ground state is different for the cuprates: it appears due to strong correlations and on-site Coulomb repulsion, and doping levels are of a different order of magnitude. However, it is interesting to see what parallels can be drawn between cuprates and semiconductors, in particular what happens when opposite carrier types are combined. That is the main focus of this thesis: the combination of electron-doped $\mathrm{Nd}_{2-\mathrm{x}} \mathrm{Ce}_{\mathrm{x}} \mathrm{CuO}_{4}$ (NCCO) with hole-doped $\mathrm{La}_{2-\mathrm{x}} \mathrm{Sr}_{\mathrm{x}} \mathrm{CuO}_{4}$ (LSCO) in different geometries. The idea of combining oppositely doped cuprates has led to theoretical predictions ranging from unconventional Josephson effects and superradiant light emission to exciton formation in bilayers leading to a variety of ordered phases, Bose-Einstein condensation and enhanced thermoelectric properties.

In chapter 1 we discuss the relevant properties of the electron and hole-doped cuprates and find that oxygen plays an important role for both types of materials. Generally, electron-doped cuprates are annealed in a vacuum after deposition and hole-doped cuprates are annealed in a high pressure oxygen environment. The role of the reduction procedure for the electron-doped cuprates is still a matter of debate, but it centers around the $\mathrm{CuO}_{2}$ planes and their local environment. Next, we discuss band alignment between NCCO and LSCO by considering them as degenerate semiconductors. Both an Anderson/Schottky-Mott type band alignment and a valence band hybridization scenario, where the oxygen $2 p$ band hybridize, are considered. Using semiconductor theory, we calculate the expected depletion zone widths and the built-in voltage energy scales for both scenarios.

In chapter 2 we investigate the properties of electron-doped NCCO under the deposition conditions for hole-doped LSCO in order to overcome the conflicting oxygenation requirements of the electron and hole-doped cuprates. We find that the copper poor, parasitic $(\mathrm{Nd}, \mathrm{Ce})_{2} \mathrm{O}_{3}$ phase plays an important role during the annealing procedure. For pulsed laser deposition (PLD), this parasitic phase is shown to be suppressed when extra copper is added to the target. Films grown from an optimally doped target with and without extra copper added were annealed using three different procedures: a standard and a long vacuum annealing and a vacuum annealing followed by an oxygen annealing suitable for the deposition of LSCO. Films grown from the target with extra copper added show a small spread in resistance, critical temperature $\left(T_{c}\right)$ and Hall coefficient, and the superconducting transition remains sharp, regardless of the annealing procedure. This is in contrast to the films grown from the target without extra 
copper, that show a large spread in properties for the different procedures. Oxygen annealing produces a strong shift towards insulating behavior, a much lower $T_{c}$ and inhomogeneous conductivity. This experiment leads to two conlusions: (1) the presence or absence of the parasitic $(\mathrm{Nd}, \mathrm{Ce})_{2} \mathrm{O}_{3}$ phase is an important factor when determining the role of oxygen in the electron doped cuprates; and (2) NCCO can be subjected to an annealing procedure suitable for the optimal growth of a holedoped cuprate like LSCO, when the parasitic $(\mathrm{Nd}, \mathrm{Ce})_{2} \mathrm{O}_{3}$ phase is suppressed by adding extra copper to the PLD target.

In chapters 3,4 and 5 we use this insight to explore the combination of electrondoped NCCO and hole-doped LSCO in the $(a, b)$-plane and in the $c$-axis direction. In the $(a, b)$-plane (chapter 3 ), this is achieved by a ramp-edge contact between NCCO and LSCO. We have fabricated junctions where both layers are superconducting, but when we measure the junctions, we do not observe a Josephson contact. Instead, we observe a strong non-linear behavior that is reminiscent of a metal-insulator-metal (MIM) tunneling contact. The tunneling nature of the contact is confirmed by the appearance of the LSCO phonon spectrum in $d V / d I$ and $d^{2} I / d V^{2}$ characteristics, signaling an elastic tunneling channel, and by the temperature dependence of the conductivity of the junctions that shows a combination of direct tunneling and inelastic tunneling via impurity states. We model the $I V$ characteristics of the junctions using a Wentzel-Kramers-Brillouin (WKB) tunneling model for a MIM contact, where we find an asymmetric barrier that is lower on the NCCO side of the interface, with a barrier height of several $10 \mathrm{~s}$ of $\mathrm{meV}$ and a width of the order of $5 \mathrm{~nm}$.

The origin of the tunneling barrier was investigated by structural analysis and by transport measurements. High-angle annular dark-field scanning transmission electron microscopy (HAADF-STEM) shows a clean interface between the NCCO and the LSCO, without any interface layers. Strain relaxation is observed in the LSCO and on the ramp, the LSCO lattice is tilted by $3^{\circ}$ with respect to the NCCO lattice. The tilting is shown to occur for the whole ramp interface across the entire junction by scanning nano-focused X-ray diffraction mapping (nXRD). Damage on the ramp as origin of the barrier is excluded by HAADF-STEM and experiments on ramp-edge homojunctions of NCCO and LSCO, where such damage is also expected. The homojunctions do show a Josephson contact and have resistances orders of magnitude lower than what we observe for the LSCO/NCCO junctions. The $3^{\circ}$ tilt of the LSCO lattice on the ramp and the resulting grain boundaries are also excluded as origin of the tunneling barrier. We argue that this tilt cannot cause the large resistance effects, because tilt grain boundaries in LSCO have shown tunneling resistances in the order of only $100 \Omega$ for angles up to $24^{\circ}$. This leaves strain and electronic effects as the possible origins of the tunneling barrier, which we further investigate by adding different overdoped interlayers to the junctions. We find a higher barrier for larger mismatch between the LSCO top layer and the interlayer, but also a decrease in the width of the barrier, regardless of the lattice mismatch with the interlayer. In the end it is difficult to disentangle the two effects and the final answer is likely a combination of the two effects, i.e. a strain effect in the LSCO and an electronic depletion effect in the NCCO. Due 
to the low barrier height, we can conclude that the most likely scenario for band alignment is a hybridization of the oxygen $2 p$ states of the valence bands of NCCO and LSCO. Other band alignment scenarios lead to an energy scale larger than what we observe for the NCCO/LSCO ramp-edge junctions.

Chapter 4 goes into more detail on the structural characterization using nXRD and HAADF-STEM and in particular the tilting of the LSCO lattice on the ramp. The lattice tilt is explained by strain accommodation, where the tilted phase has a lower mismatch with the exposed facets on the ramp than a $c$-axis aligned phase. This can be described by a model that takes into account the lattice mismatch between the two materials and the $a / c$-axis length ratio. This model shows a lattice tilt that depends on the ramp angle, where for a range of ramp angles, the tilted phase is favored over $c$-axis alignment. We have applied this model to other oxide systems like $\mathrm{SrTiO}_{3}, \mathrm{BiFeO}_{3}$ and $\mathrm{PbTiO}_{3}$, where the model is able to describe lattice tilting across domain boundaries between different crystal phases. This leads us to two conclusions: (1) there is a crystalline contact between NCCO and LSCO at the ramp interface, and (2) ramp interfaces can potentially be used for strain engineering; for materials that show multiple crystal phases, varying the ramp angle can promote the nucleation of one of the phases.

In chapter 5 we investigate the combination of NCCO and LSCO in a bilayer configuration. For this, we have developed a deposition scheme to fabricate NCCOLSCO bilayers, both with NCCO and LSCO as bottom layer. Intermediate annealing steps with a cooling step down to $600^{\circ} \mathrm{C}$ are employed, using vacuum annealing when NCCO is used as the bottom layer and 1 bar oxygen annealing when LSCO is the bottom layer. In both cases we show that both the NCCO and the LSCO are superconducting. The configuration where NCCO is the bottom layer is investigated further using HAADF-STEM and by $c$-axis tunneling measurement. Here we find that the interface is rough and not well defined, due to the intrinsic roughness of the NCCO layer. In $c$-axis transport we observe a similar barrier as for the in-plane junction, but here the barrier appears to be wider. This is to be expected from the longer screening length in the $c$-axis direction. Due to a large contact resistance to the LSCO layer, the bias range for the $c$-axis transport measurements is limited and the barrier cannot be characterized in more detail.

The electronic structure of LSCO in the bilayer structure was investigated by X-ray absorption spectroscopy (XAS). Measurements were performed near the oxygen $K$-edge and the copper $L_{2,3}$-edge. A bilayer with LSCO as top layer and reference films of LSCO and NCCO were measured. XAS has a limited probe depth and therefore only the LSCO layer in the bilayer was measured. There is a global agreement between the spectra for LSCO in the bilayer and the reference, with two major differences: in the bilayer, the Zhang-Rice singlet peak, characteristic of hole doping in the cuprates, is suppressed or shifted in the $\mathrm{O} K$-edge spectra and an additional peak, attributed to $\mathrm{Cu}^{1+}$, is observed in the $\mathrm{Cu} L_{2,3}$-edge spectra. HAADF-STEM, X-ray diffraction and atomic force microscopy are used to exclude an impurity phase as origin of the $\mathrm{Cu}^{1+}$ signal. The signal is suggestive of an electron doping effect in the LSCO layer that is different from what is observed 
for the electron-doped cuprates. Further research is needed to exactly identify the origin of the $\mathrm{Cu}^{1+}$ signal and its precise location in the LSCO film.

In the final chapter, chapter 6 , we discuss the first experiments towards a test for current quantization in type-II Bose-Mott insulator. This work is based on a theoretical prediction that there is a dual to the type-II superconductor, the type-II Bose-Mott insulator, that is connected via quantum disorder. In the superconducting state, order is broken by disorder in the form of vortices. The BoseMott insulator phase can be described in a similar way, but here it is the vortices that form the ordered state. Exploiting this connection, it has been predicted that the Bose-Mott insulator phase has certain properties that mirror those of the superconducting phase. Most importantly, a type-II Bose-Mott insulator hosting current lines (current quanta) as a dual to magnetic vortices (flux quanta) in a type-II superconductor is predicted. A prime candidate for a type-II Bose-Mott insulator is the pseudogap phase of the hole-doped cuprates, in this case underdoped $\mathrm{La}_{2-\mathrm{x}} \mathrm{Sr}_{\mathrm{x}} \mathrm{CuO}_{4}$. This phase is theorized to be comprised of preformed Cooper pairs with no long-range order, which could form the Bose-Mott insulator state. For these materials, a single current quantum is predicted to be around $1.4 \mathrm{~mA}$. We have fabricated devices where a thin layer of underdoped $\mathrm{La}_{1.95} \mathrm{Sr}_{0.05} \mathrm{CuO}_{4}$ is sandwiched between optimally doped, superconducting LSCO in the form of single ramp-edge junctions and junctions embedded in a superconducting ring. The latter should reveal the presence of the current quantum in its magnetization. In the measurement of the temperature dependence of the resistance of the single junctions, we recover the known properties of $\mathrm{La}_{1.95} \mathrm{Sr}_{0.05} \mathrm{CuO}_{4}$ : the junction shows variable range hopping in transport, characteristic of the spin glass phase of underdoped $\mathrm{La}_{2-\mathrm{x}} \mathrm{Sr}_{\mathrm{x}} \mathrm{CuO}_{4}$ at low temperatures. This is also reflected in the $I V$ characteristics, where we find an inelastic tunneling contact that corresponds to a superconductor-insulator-normal metal-superconductor (SINS) contact. The experiments show no evidence of the proposed properties of the candidate type-II Bose-Mott insulator, but have been limited by the critical current of the superconducting leads. Future experiments will focus on junctions with higher critical current densities, to ensure multiple current quanta can be carried by the leads, and on alternative measurement geometries. 


\section{Samenvatting}

Er zijn veel parallellen te trekken tussen halfgeleiders en hoge-temperatuur supergeleidende cupraten, zowel gezien vanuit een historisch als natuurkundig perspectief. Beide worden gekenmerkt door een isolerende grondtoestand en worden geleidend door dotering. De oorsprong van de grondtoestand is anders voor de cupraten, ze ontstaat door sterke correlaties en Coulomb afstoting op roosterposities, en het doteringsniveau is van een andere orde grootte. Desalniettemin is het interessant om te kijken welke parallellen er getrokken kunnen worden tussen cupraten en halfgeleiders, in het bijzonder wat er gebeurt als ladingsdragers met verschillend teken gecombineerd worden. Dit is het hoofdonderwerp van dit proefschrift: het bestuderen van de combinatie van elektronen-gedoteerd $\mathrm{Nd}_{2-\mathrm{x}} \mathrm{Ce}_{\mathrm{x}} \mathrm{CuO}_{4}$ (NCCO) en gaten-gedoteerd $\mathrm{La}_{2-\mathrm{x}} \mathrm{Sr}_{\mathrm{x}} \mathrm{CuO}_{4}$ (LSCO) in verschillende geometrieën. Het idee om verschillende typen ladingsdragers te combineren in cupraten heeft al geleid tot theoretische voorspellingen variërend van ongebruikelijke Josephson effecten en superradiante emissie tot exciton vorming in bilagen, wat kan leiden tot verschillende geordende fases, Bose-Einstein condensatie en verbeterde thermo-elektrische eigenschappen.

In hoofdstuk 1 bespreken we de relevante eigenschappen van de elektronen- en gaten-gedoteerde cupraten ( $n$-type en $p$-type) en zien we dat zuurstof een belangrijk rol speelt voor beide typen materialen. In het algemeen worden $n$-type cupraten na depositie in vacuüm gehouden, terwijl $p$-type cupraten typisch uitgebakken worden in zuurstof. De precieze rol van de zuurstof reductie van de $n$-type cupraten staat nog steeds ter discussie, maar het draait om de $\mathrm{CuO}_{2}$ vlakken en hun lokale omgeving. Vervolgens bespreken we de banduitlijning tussen NCCO en LSCO door ze te beschouwen als ontaarde halfgeleiders. We bekijken zowel een Anderson/Schottky-Mott banduitlijning alsook een uitlijning waarbij we uitgaan van een hybridisatie van de zuurstof $2 p$ toestanden in de valentiebanden. Met behulp van halfgeleidertheorie berekenen we de breedtes van de uitputtingszones en de energieschalen van de ingebouwde spanningen.

In hoofdstuk 2 onderzoeken we de eigenschappen van de $n$-type cupraat NCCO terwijl we de depositie instelling gebruiken voor de $p$-type cupraat LSCO. Hierbij hebben we ontdekt dat een koperarme, parasitaire fase, $(\mathrm{Nd}, \mathrm{Ce})_{2} \mathrm{O}_{3}$, een belangrijke rol speelt tijdens de warmtebehandeling. Bij de groei van NCCO dunne films met gepulseerde laser depositie (PLD) wordt deze fase wordt onderdrukt als er extra koper aan het target wordt toegevoegd. Films gegroeid van optimaal gedoteerde targets, met en zonder extra koper, zijn afgekoeld met drie verschillende warmtebehandelingen: een standaard en een lange vacuümbehandeling en een behandeling waarbij vacuüm gevolgd wordt door een behandeling in zuurstof, geschikt voor LSCO. Films gegroeid van het target met extra koper toegevoegd 
hebben een kleinere spreiding in weerstand, kritische temperatuur $\left(T_{c}\right)$ en Hall coëfficiënt, en de supergeleidende overgang blijft scherp, ongeacht welke warmtebehandeling gebruikt wordt. De films gegroeid van het target zonder extra koper laten daarentegen een grote spreiding in eigenschappen zien. Met name de behandeling in zuurstof zorgt voor een sterke verschuiving richting de isolerende grondtoestand, een sterke onderdrukking van $T_{c}$ en inhomogene geleiding. Uit dit experiment kunnen twee zaken geconcludeerd worden: (1) de aanwezigheid van de parasitaire $(\mathrm{Nd}, \mathrm{Ce})_{2} \mathrm{O}_{3}$ fase is een belangrijke factor voor het bepalen van de rol van zuurstof in de elektronen-gedoteerde cupraten; en (2) NCCO kan blootgesteld worden aan een afkoelprocedure geschikt voor de groei van LSCO wanneer extra koper aan het PLD target is toegevoegd.

In hoofdstukken 3,4 en 5 gebruiken we deze kennis om de combinatie van $n$-type en $p$-type cuprate verder te onderzoeken door combinaties te maken in het $(a, b)$-vlak en in de $c$-as richting. In het $(a, b)$-vlak (hoofdstuk 3 ) doen we dit door middel van een helling-type (ramp-edge) junctie tussen NCCO en LSCO. We hebben juncties gemaakt waarbij beide lagen supergeleidend zijn, maar wanneer we de juncties meten, meten we geen Josephson contact. In tegendeel, we zien een sterk nietlineaire $I V$ karakteristiek die duidt op een metaal-isolator-metaal tunnelcontact. Het tunnelkarakter van het contact wordt bevestigd door het verschijnen van het LSCO fononspectrum in $d V / d I$ en $d^{2} V / d I^{2}$ karakteristieken, wat wijst op elastisch tunnelen, en door de temperatuursafhankelijkheid die een combinatie laat zien van elastisch en inelastisch tunnelen. We hebben daarom de $I V$ karakteristieken gemodelleerd met een Wentzel-Kramers-Brillouin (WKB) tunnel model voor een metaal-isolator-metaal contact, waarbij we een asymmetrische barrière vinden die lager is aan de NCCO zijde van het grensvlak met een hoogte van enkele tientallen $\mathrm{meV}$ en een breedte in de orde van $5 \mathrm{~nm}$.

De oorsprong van de tunnelbarrière is onderzocht met behulp van structurele analyse en transport metingen. High-angle annular dark-field scanning transmission electron microscopy (HAADF-STEM, raster transmissie-elektronenmicroscopie) toont een schoon grensvlak tussen NCCO en LSCO, zonder een grensvlaklaag van een ander materiaal. In het LSCO is een relaxatie van de roosterspanning te zien en het LSCO rooster is drie graden gekanteld ten opzichte van het NCCO rooster. Deze kanteling vindt alleen op de helling plaats die in het NCCO is geëtst. Deze kanteling is niet lokaal, maar strekt zich uit over de hele breedte van de junctie, wat is aangetoond met behulp van nanofocus Röntgen diffractie (nano-focused $X$-ray diffraction, nXRD), waarmee het gebied rond het grensvlak in kaart is gebracht. Beschadiging van de helling tijdens de fabricage als oorzaak van de tunnelbarrière kan worden uitgesloten met behulp van HAADF-STEM en experimenten met homojuncties van LSCO en NCCO, waar vergelijkbare schade verwacht wordt. Deze juncties laten wél een Josephson contact zien en deze juncties hebben een weerstand ordes van grootte kleiner dan de weerstand van de LSCO/NCCO juncties. De drie graden kanteling van het LSCO rooster en de bijbehorende korrelgrenzen kunnen ook uitgesloten worden als oorzaak van de tunnelbarrière. We beargumenteren dat deze kanteling niet een dusdanig grote weerstand kan veroorzaken aangezien korrelgrenzen in LSCO met kantelhoeken 
tot $24^{\circ}$ tunnelweerstanden laten zien van de orde $100 \Omega$. Dit betekent dat alleen roosterspanning en elektronische effecten overblijven als mogelijke oorzaken van de tunnelbarrière, wat we onderzoeken door te kijken naar het effect van verschillende overgedoteerde tussenlagen. We vinden een hogere barrière voor een hogere roosterspanning tussen LSCO en de tussenlaag, maar de barrière is ook smaller bij het gebruik van een tussenlaag, ongeacht de roosterspanning. Uiteindelijk zijn deze twee effecten lastig te ontwarren en het uiteindelijke antwoord is waarschijnlijk een combinatie van de beide effecten, dus een effect door de roosterspanning in het LSCO en een elektronische uitputtingszone in het NCCO. Vanwege de lage barrièrehoogte kunnen we ook concluderen dat de meest waarschijnlijke banduitlijning hybridisatie van de valentiebanden is. Andere scenario's geven een energieschaal die te groot is voor wat we meten.

Hoofdstuk 4 bespreekt de structurele karakterisatie met behulp van HAADFSTEM en nXRD in meer detail. In het bijzonder is er aandacht voor de kanteling van het LSCO rooster op de NCCO helling. De kanteling wordt veroorzaakt door roosterspanning. Het blijkt dat het gekantelde rooster een lagere roosterspanning ondervindt wanneer het de facetten van NCCO volgt die blootliggen op de helling, in plaats van met de $c$-as uit te lijnen. Dit kan beschreven worden met een geometrisch model dat de roosterspanning en de $a / c$-as lengteverhouding meeneemt. Dit model geeft een roosterkanteling die afhangt van de hellinghoek en waarbij voor een bepaald bereik aan hellinghoeken de gekantelde fase energetisch voordeliger is dan de normale $c$-as groei. We hebben dit model ook toegepast op andere oxide systemen zoals $\mathrm{SrTiO}_{3}, \mathrm{BiFeO}_{3}$ en $\mathrm{PbTiO}_{3}$, waar het model ook de kantelhoek kan beschrijven voor korrel- en domeingrenzen tussen verschillende kristalfasen. Dit heeft geleid tot twee conclusies: (1) er is een kristallijn contact tussen NCCO en LSCO in de helling-type juncties en (2) dit type juncties kan mogelijk gebruikt worden voor strain engineering; voor materialen die in verschillende kristalfasen kunnen groeien, afhankelijk van de roosterspanning met het substraat, kan het gebruik van een hellinghoek de groei van een van de verschillende fasen bevorderen.

In hoofdstuk 5 onderzoeken we de combinatie van NCCO en LSCO in een bilaag, een contact in de $c$-as richting. Hiervoor is een depositierecept ontwikkeld voor het maken van bilagen, zowel met NCCO als met LSCO als onderste laag. Tussen de depositie van de twee lagen is een warmtebehandelingsstap toegevoegd, waarbij afgekoeld wordt tot $600^{\circ} \mathrm{C}$. Voor NCCO wordt de film tijdens deze stap in vacuüm gehouden en voor LSCO in 1 bar zuurstof. In beide configuraties laten we zien dat zowel NCCO als LSCO supergeleidend zijn. De variant met NCCO als onderste laag is verder onderzocht met behulp van HAADF-STEM en $c$-as tunnelmetingen. Met HAADF-STEM vinden we een ruw, slecht gedefinieerd grensvlak, veroorzaakt door de intrinsieke ruwheid van de NCCO laag. De $c$-as transportmetingen laten een vergelijkbare barrière zien als voor de juncties beschreven in hoofdstuk 3 , maar de barrière lijkt breder te zijn. In deze metingen is het spanningsbereik beperkt door hoge contactweerstanden naar het LSCO toe, hierdoor kan de barrière niet in meer detail worden gekarakteriseerd.

De elektronische structuur van het LSCO in de bilaag is onderzocht met behulp 
van Röntgen absorptie spectroscopie (XAS, X-ray absorption spectroscopy). Deze metingen zijn gedaan voor de zuurstof $K$-lijn en de koper $L_{2,3}$-lijn aan een bilaag met LSCO als toplaag en aan referentiefilms van NCCO en LSCO. XAS heeft een beperkte penetratiediepte, dus alleen de LSCO toplaag wordt gemeten bij de bilaag. De gemeten spectra voor LSCO in de bilaag en de referentiefilm komen globaal overeen, met twee grote verschillen: in de bilaag, in de zuurstof spectra is het Zhang-Rice singlet, wat karakteristiek is voor gaten-dotering in cupraten, onderdrukt of verschoven en in de koper spectra is een extra piek verschenen, afkomstig van $\mathrm{Cu}^{1+}$. Met behulp van HAADF-STEM, Röntgen diffractie en atoomkrachtmicroscopie kunnen we uitsluiten dat het $\mathrm{Cu}^{1+}$ signaal afkomstig is van een vervuiling of een andere fase in de bilaag. Het signaal suggereert dat er een elektronen-dotering plaatsvindt in het LSCO en dat dit verschilt van de elektronen-dotering in de $n$-type cupraten. Meer onderzoek is nodig om de exacte oorsprong van het $\mathrm{Cu}^{1+}$ signaal te achterhalen.

In het laatste hoofdstuk, hoofdstuk 6 , bespreken we de eerste experimenten om stroomkwantisatie in type-II Bose-Mott isolatoren te testen. Dit werk is gebaseerd op een theoretische voorspelling dat er een duale toestand is voor de type-II supergeleider, de type-II Bose-Mott isolator, verbonden via kwantumwanorde. In de supergeleidende fase wordt de orde verbroken door wanorde in de vorm van vortices. De Bose-Mott isolator toestand kan op een vergelijkbare manier beschreven worden, maar hier zijn het de vortices die de geordende toestand vormen. Door deze verbinding te gebruiken is er voorspeld dat de Bose-Mott isolator toestand eigenschappen vertoont die het spiegelbeeld zijn van die van de supergeleidende fase. In het bijzonder is er voorspeld dat een type-II Bose-Mott isolator stroomlijnen (stroomkwanten) kan bevatten, analoog aan de magnetische vortices (fluxkwanten) in de type-II supergeleider. De pseudogap fase van de $p$-type cupraten is een goede kandidaat Bose-Mott isolator, in ons geval $\mathrm{La}_{1.95} \mathrm{Sr}_{0.05} \mathrm{CuO}_{4}$. Van deze fase is theoretisch voorspeld dat ze bestaat uit voorgevormde Cooperparen die nog geen globale ordening vertonen, wat past bij de beschrijving van de BoseMott isolator. Voor deze materialen zou het stroomkwantum ongeveer $1.4 \mathrm{~mA}$ zijn. Wij hebben structuren gemaakt waarbij een dunne laag van ondergedoteerd $\mathrm{La}_{1.95} \mathrm{Sr}_{0.05} \mathrm{CuO}_{4}$ geplaatst is tussen optimaal gedoteerd, supergeleidend LSCO in de vorm van een enkele helling-type junctie en als een junctie in een gesloten supergeleidende ring. De laatste zou de stroomkwanten moeten laten zien in een meting van de magnetisatie. Bij het meten de temperatuursafhankelijkheid van de weerstand van de losse juncties vinden we de reeds bekende eigenschappen van $\mathrm{La}_{1.95} \mathrm{Sr}_{0.05} \mathrm{CuO}_{4}$ : de juncties laten variable range hopping zien, karakteristiek voor de spinglas fase van ondergedoteerd $\mathrm{La}_{2-\mathrm{x}} \mathrm{Sr}_{\mathrm{x}} \mathrm{CuO}_{4}$ op lage temperaturen. Dit komt ook terug in de $I V$ karakteristieken, hier vinden we een inelastisch tunnelcontact dat overeenkomt met een supergeleider-isolator-normaal metaalsupergeleider (SINS) contact. De experimenten laten geen van de voorspelde eigenschappen van de type-II Bose-Mott isolator zien. In de toekomst zullen experimenten zich richten op juncties met een hogere kritische stroomdichtheid, zodat meerdere stroomkwanten gedragen kunnen worden door de supergeleidende elektrodes, en op alternatieve meetgeometrieën. 


\section{Dankwoord}

Met veel plezier heb ik het afgelopen decennium doorgebracht in Twente. Dit proefschrift is een mooie afsluiter van deze periode en van een interessant promotietraject van vier jaar en een beetje. Natuurlijk heb ik dat niet zonder hulp gedaan, dus hierbij wil ik graag een aantal mensen bedanken.

In de eerste plaats wil ik Hans bedanken voor het formuleren van een interessante opdracht, de begeleiding en voor vertrouwen en vrijheid tijdens het uitvoeren van het onderzoek.

Secondly, I thank Francesco, for being my 'partner in crime' on the VICI project. I have really enjoyed working together, our discussions and our numerous trips to conferences and summer schools.

Next, I would like to thank Renshaw for a wonderful collaboration and for having the uncanny knack of catching me for an hour-long discussion, right when I am about to leave. You have really helped the interpretation of the experiments by asking the right questions.

This work would not have been possible without some great input and experiments from collaborators. I would like to thank Nicola, Renshaw and the people at ESRF for the nano-focused XRD experiments of chapter 4, Xiaoxing Ke and professor van Tendeloo and the people from the University of Antwerp for the HAADFSTEM measurements in chapters 3,4 and 5, Andrivo Rusydi and his students for the X-ray absorption spectroscopy measurement and Rico Keim for the TEM measurements in chapter 2 .

Daarnaast wil ik graag Louk bedanken voor de interessante discussies en conferentiebezoeken, alsook voor zijn bereidheid om al mijn theorievragen te beantwoorden; Jan Zaanen voor de gastvrijheid in Leiden en de stimulerende bijeenkomsten met de stripe-club; en Jan van Ruitenbeek en Hasan voor de samenwerking die heeft geleid tot hoofdstuk 6 , ik hoop dat we de stroomkwanten ooit gaan vinden!

Een dankwoord bij ICE zou niet compleet zijn zonder een groot woord van dank aan Frank en Dick, zonder hen zou het hele lab uit elkaar vallen. Het was altijd gezellig om met jullie samen te werken en het spijt me dat ik zo vaak de krant meenam.

Tijdens haar afstuderen heeft Denise een belangrijke bijdrage geleverd aan mijn onderzoek en ook de bacheloropdracht van Florian en Sander heeft tot de nodige nieuwe inzichten geleid, waarvoor dank.

Ondanks dat mijn opdracht niets met topological insulators te maken had, is het toch een thema geweest tijdens mijn promotie. Dit heb ik vooral te danken aan 
Menno, die mij na mijn afstuderen toch steeds weer verleidde met een interessante vraag of een experiment. Dit heeft geleid tot een aantal mooie publicaties, dus daarvoor mijn dank aan Menno, Alexander en het hele topo-team.

Een proefschrift schrijf je in principe alleen, maar met alleen de spellingcontrole van Texmaker kom je er niet. Ik wil daarom Francesco, Sebastiaan, Denise, Menno, Aron, Hans, Cor en mijn moeder bedanken voor het lezen van (stukken van) mijn proefschrift en voor de nuttige commentaren.

Natuurlijk wil ik ook iedereen bij ICE en de collega's binnen MESA+ bedanken voor een geweldige tijd, in het bijzonder de WhatsApp-koffiegroep voor de vele koffie en puzzels, Cor, Sebastiaan, Hidde en Frank voor de vele fietstochten en Cor en Diederik voor de lol in het nieuwe lab met Hoek-Jekel-Molenaar installatie B.V.

Tenslotte wil ik al mijn familie en vrienden bedanken en in het bijzonder mijn ouders en mijn broertje.

Marcel Hoek

Enschede, juli 2014 


\section{References}

[1] J. Bednorz and K. Müller, Z. Phys. B 64(2) 189-193 (1986)

[2] M. K. Wu, J. R. Ashburn, C. J. Torng, P. H. Hor, R. L. Meng, L. Gao, Z. J. Huang, Y. Q. Wang and C. W. Chu, Phys. Rev. Lett. 58 908-910 (1987)

[3] J. Zaanen, 100 years of superconductivity, A modern, but way too short history of the theory of superconductivity at high temperature, Taylor \& Francis (2012)

[4] J. Heber, Nature 459 28-30 (2009), (News Feature)

[5] H. Y. Hwang, Y. Iwasa, M. Kawasaki, B. Keimer, N. Nagaosa and Y. Tokura, Nat. Mater. 11(2) 103-113 (2012)

[6] A. Ohtomo and H. Y. Hwang, Nature 427(6973) 423-426 (2004)

[7] K. v. Klitzing, G. Dorda and M. Pepper, Phys. Rev. Lett. 45 494-497 (1980)

[8] D. C. Tsui, H. L. Stormer and A. C. Gossard, Phys. Rev. Lett. 48 1559-1562 (1982)

[9] M. Huijben, G. Koster, M. K. Kruize, S. Wenderich, J. Verbeeck, S. Bals, E. Slooten, B. Shi, H. J. A. Molegraaf, J. E. Kleibeuker, S. van Aert, J. B. Goedkoop, A. Brinkman, D. H. A. Blank, M. S. Golden, G. van Tendeloo, H. Hilgenkamp and G. Rijnders, Adv. Funct. Mater. 23(42) 5240-5248 (2013)

[10] J. Mannhart, A. Kleinsasser, J. Ströbel and A. Baratoff, Physica C Supercond. 216(3-4) 401-416 (1993)

[11] J. Hu, C. Wu and X. Dai, Phys. Rev. Lett. 99067004 (2007)

[12] M. Charlebois, S. R. Hassan, R. Karan, D. Sénéchal and A.-M. S. Tremblay, Phys. Rev. B 87(3) 035137 (2013)

[13] L. Rademaker, K. Wu, H. Hilgenkamp and J. Zaanen, Europhys. Lett. 97(2) 27004 (2012)

[14] L. Rademaker, J. van den Brink, H. Hilgenkamp and J. Zaanen, Phys. Rev. B 88121101 (2013)

[15] L. Rademaker, J. van den Brink, J. Zaanen and H. Hilgenkamp, Phys. Rev. B 88235127 (2013)

[16] K. Wu, L. Rademaker and J. Zaanen, arXiv:cond-mat 1401.7770 (2014)

[17] A. A. High, J. R. Leonard, M. Remeika, L. V. Butov, M. Hanson and A. C. Gossard, Nano Lett. 12(5) 2605-2609 (2012) 
[18] A. Croxall, K. Das Gupta, C. Nicoll, I. Farrer, H. Beere, D. Ritchie and M. Pepper, Physica E 42(4) 1247-1250 (2010)

[19] J. P. Eisenstein and A. H. MacDonald, Nature 432(7018) 691-694 (2004)

[20] Y. Yoon, L. Tiemann, S. Schmult, W. Dietsche, K. von Klitzing and W. Wegscheider, Phys. Rev. Lett. 104(11) 116802 (2010)

[21] X. Huang, W. Dietsche, M. Hauser and K. von Klitzing, Phys. Rev. Lett. 109(15) 156802- (2012)

[22] J. Kasprzak, M. Richard, S. Kundermann, A. Baas, P. Jeambrun, J. M. J. Keeling, F. M. Marchetti, M. H. Szymanska, R. Andre, J. L. Staehli, V. Savona, P. B. Littlewood, B. Deveaud and L. S. Dang, Nature 443(7110) 409-414 (2006)

[23] H. Deng, H. Haug and Y. Yamamoto, Rev. Mod. Phys. 82(2) 1489-1537 (2010)

[24] B. Nelsen, G. Liu, M. Steger, D. W. Snoke, R. Balili, K. West and L. Pfeiffer, Phys. Rev. X 3(4) 041015 (2013)

[25] L. Rademaker, K. Wu and J. Zaanen, New J. Phys. 14(8) 083040 (2012)

[26] I. Tsukada, Phys. Rev. B 64(22) 224501 (2001)

[27] A. Tsukada, T. Greibe and M. Naito, Phys. Rev. B 66(18) 184515 (2002)

[28] L. Zhao, R. Liu, G. Wu, G. Wang, T. Wu, X. Luo and X. Chen, Supercond. Sci. Technol. 21(6) 065005 (2008)

[29] N. P. Armitage, P. Fournier and R. L. Greene, Rev. Mod. Phys. 82(3) 2421 (2010)

[30] J. Zaanen, G. A. Sawatzky and J. W. Allen, Phys. Rev. Lett. 55(4) 418 (1985)

[31] P. G. Radaelli, J. D. Jorgensen, A. J. Schultz, J. L. Peng and R. L. Greene, Phys. Rev. B 49 15322-15326 (1994)

[32] Y. Tokura, H. Takagi and S. Uchida, Nature 337(6205) 345-347 (1989)

[33] H. Takagi, S. Uchida and Y. Tokura, Phys. Rev. Lett. 62 1197-1200 (1989)

[34] J. Kim and D. Gaskell, Physica C Supercond. 209(4) 381-388 (1993)

[35] E. Navarro, D. Jaque, J. Villegas, J. Martín, A. Serquis, F. Prado, A. Caneiro and J. Vicent, J. Alloys Compd. 323-324 580-583 (2001)

[36] P. K. Mang, S. Larochelle, A. Mehta, O. P. Vajk, A. S. Erickson, L. Lu, W. J. L. Buyers, A. F. Marshall, K. Prokes and M. Greven, Phys. Rev. B 70094507 (2004)

[37] H. J. Kang, P. Dai, B. J. Campbell, P. J. Chupas, S. Rosenkranz, P. L. Lee, Q. Huang, S. Li, S. Komiya and Y. Ando, Nat. Mater. 6(3) 224-229 (2007)

[38] H. Kimura, Y. Noda, F. Sato, K. Tsuda, K. Kurahashi, T. Uefuji, M. Fujita and K. Yamada, J. Phys. Soc. Jpn. 74(8) 2282-2286 (2005) 
[39] A. Tsukada, Y. Krockenberger, M. Noda, H. Yamamoto, D. Manske, L. Alff and M. Naito, Solid State Commun. 133(7) 427-431 (2005)

[40] L. Zhao, G. Wu, R. H. Liu and X. H. Chen, Appl. Phys. Lett. 90(7) 072503 (2007)

[41] Y. Krockenberger, H. Irie, O. Matsumoto, K. Yamagami, M. Mitsuhashi, A. Tsukada, M. Naito and H. Yamamoto, Sci. Rep. 32235 (2013)

[42] W. Jiang, J. L. Peng, Z. Y. Li and R. L. Greene, Phys. Rev. B 47(13) 8151 (1993)

[43] A. J. Schultz, J. D. Jorgensen, J. L. Peng and R. L. Greene, Phys. Rev. B $535157-5159$ (1996)

[44] X. Q. Xu, S. N. Mao, W. Jiang, J. L. Peng and R. L. Greene, Phys. Rev. B $53871-875$ (1996)

[45] J. S. Higgins, Y. Dagan, M. C. Barr, B. D. Weaver and R. L. Greene, Phys. Rev. B 73(10) 104510 (2006)

[46] G. Riou, P. Richard, S. Jandl, M. Poirier, P. Fournier, V. Nekvasil, S. N. Barilo and L. A. Kurnevich, Phys. Rev. B 69024511 (2004)

[47] P. Richard, G. Riou, I. Hetel, S. Jandl, M. Poirier and P. Fournier, Phys. Rev. B 70064513 (2004)

[48] J. Gauthier, S. Gagné, J. Renaud, M.-E. Gosselin, P. Fournier and P. Richard, Phys. Rev. B 75024424 (2007)

[49] D. Song, S. R. Park, C. Kim, Y. Kim, C. Leem, S. Choi, W. Jung, Y. Koh, G. Han, Y. Yoshida, H. Eisaki, D. H. Lu, Z.-X. Shen and C. Kim, Phys. Rev. $B 86144520$ (2012)

[50] F. Prado, J. Briático, A. Caneiro, M. Tovar and M. Causa, Solid State Commun. 90(11) 695-699 (1994)

[51] A. Seffar, J. Fontcuberta, S. Piñol, X. Obradors, G. Peraudeau and R. Berjoan, Physica C Supercond. 259(1-2) 75-82 (1996)

[52] K. Kurahashi, H. Matsushita, M. Fujita and K. Yamada, J. Phys. Soc. Jpn. 71(3) 910-915 (2002)

[53] A. Gupta, R. Gross, E. Olsson, A. Segm ller, G. Koren and C. C. Tsuei, Phys. Rev. Lett. 64(26) 3191 (1990)

[54] S. N. Mao, X. X. Xi, Q. Li, I. Takeuchi, S. Bhattacharya, C. Kwon, C. Doughty, A. Walkenhorst, T. Venkatesan, C. B. Whan, J. L. Peng and R. L. Greene, Appl. Phys. Lett. 62(19) 2425-2427 (1993)

[55] I. Takeuchi, S. N. Mao, X. X. Xi, K. Petersen, C. J. Lobb and T. Venkatesan, Appl. Phys. Lett. 67(19) 2872-2874 (1995)

[56] H. Haensel, A. Beck, F. Gollnik, R. Gross, R. Huebener and K. Knorr, Physica C Supercond. 244(3-4) 389-399 (1995) 
[57] L. Alff, U. Schoop, R. Gross, R. Gerber and A. Beck, Physica C Supercond. 271(3-4) 339-348 (1996)

[58] A. Kussmaul, J. S. Moodera, P. M. Tedrow and A. Gupta, Appl. Phys. Lett. 61(22) 2715-2717 (1992)

[59] F. Kaufman, J. Chem. Phys. 46 2449-2449 (1967)

[60] P. Brix and G. Herzberg, J. Chem. Phys. 21(12) 2240-2240 (1953)

[61] C. Wu, M. Wang and M. Wu, Physica C Supercond. 460-462(Part 1) 424425 (2007)

[62] P. Fournier, private communication (2013)

[63] M. Naito and M. Hepp, Jpn. J. Appl. Phys. 39(2 6A) L485-L487 (2000)

[64] A. Sawa, M. Kawasaki, H. Takagi and Y. Tokura, Phys. Rev. B 66(1) 014531 (2002)

[65] A. Tsukada, H. Yamamoto and M. Naito, Phys. Rev. B 74(17) 174515 (2006)

[66] J. Felsche, Naturwissenschaften 56212 (1969)

[67] J. R. Rustad, Am. Mineral. 97(5-6) 791-799 (2012)

[68] A. Inam, C. T. Rogers, R. Ramesh, K. Remschnig, L. Farrow, D. Hart, T. Venkatesan and B. Wilkens, Appl. Phys. Lett. 57(23) 2484-2486 (1990)

[69] L. Zhao, H. Wu, J. Miao, H. Yang, F. C. Zhang, X. G. Qiu and B. R. Zhao, Supercond. Sci. Technol. 17(11) 1361 (2004)

[70] W. Haynes, editor, CRC Handbook of Chemistry and Physics, chapter 12, 11-12, CRC press (2013-2014)

[71] H. Palmer, C. Greaves, M. Slaski, V. Trofimova, Y. Yarmoshenko and E. Kurmaev, Physica C Supercond. 291(1-2) 104-112 (1997)

[72] V. E. Henrich, G. Dresselhaus and H. J. Zeiger, Phys. Rev. B 17 4908-4921 (1978)

[73] D. Kan, O. Sakata, S. Kimura, M. Takano and Y. Shimakawa, Jpn. J. Appl. Phys. 46 L471-473 (2007)

[74] H. Gross, N. Bansal, Y.-S. Kim and S. Oh, J. Appl. Phys. 110(7) 073704 (2011)

[75] CrysTec GmbH, crystec.de

[76] T. Ohnishi, K. Takahashi, M. Nakamura, M. Kawasaki, M. Yoshimoto and H. Koinuma, Appl. Phys. Lett. 74(17) 2531-2533 (1999)

[77] P. Brinks, W. Siemons, J. E. Kleibeuker, G. Koster, G. Rijnders and M. Huijben, Appl. Phys. Lett. 98(24) 242904 (2011)

[78] J. P. Shi, Y. G. Zhao, H. J. Zhang, H. F. Tian and X. P. Zhang, Appl. Phys. Lett. 94(19) 192103 (2009) 
[79] B.-S. Li, A. Sawa and H. Okamoto, Appl. Phys. Lett. 102(11) 111606 (2013)

[80] S. M. Sze, Physics of Semiconductor Devices, Wiley-Interscience (1969)

[81] H. B. Michaelson, J. Appl. Phys. 48(11) 4729-4733 (1977)

[82] M. Kofu, H. Kimura, H. Ishida, T. Matsumura and K. Hirota, Phys. Rev. B 79184508 (2009)

[83] R. Daou, N. Doiron-Leyraud, D. LeBoeuf, S. Y. Li, F. Laliberte, O. CyrChoiniere, Y. J. Jo, L. Balicas, J.-Q. Yan, J.-S. Zhou, J. B. Goodenough and L. Taillefer, Nat Phys 5(1) 31-34 (2009)

[84] S. Yunoki, A. Moreo, E. Dagotto, S. Okamoto, S. S. Kancharla and A. Fujimori, Phys. Rev. B 76(6) 064532 (2007)

[85] N. Harima, J. Matsuno, A. Fujimori, Y. Onose, Y. Taguchi and Y. Tokura, Phys. Rev. B 64(22) 220507 (2001)

[86] Y. Sakisaka, T. Maruyama, Y. Morikawa, H. Kato, K. Edamoto, M. Okusawa, Y. Aiura, H. Yanashima, T. Terashima, Y. Bando, K. Iijima, K. Yamamoto and K. Hirata, Solid State Commun. 74(7) 609-611 (1990)

[87] A. Fujimori, A. Ino, J. Matsuno, T. Yoshida, K. Tanaka and T. Mizokawa, J. Electron Spectrosc. Relat. Phenom. 124(2-3) 127-138 (2002)

[88] L. H. Thomas, Math. Proc. Camb. Phil. Soc. 23 542-548 (1927)

[89] E. Fermi, Rend. Accad. Naz. Lincei 6 602-607 (1927)

[90] C. Y. Chen, R. J. Birgeneau, M. A. Kastner, N. W. Preyer and T. Thio, Phys. Rev. B 43(1) 392 (1991)

[91] M. Weger, J. Supercond. 10(4) 435-440 (1997)

[92] C. H. Ahn, A. Bhattacharya, M. Di Ventra, J. N. Eckstein, C. D. Frisbie, M. E. Gershenson, A. M. Goldman, I. H. Inoue, J. Mannhart, A. J. Millis, A. F. Morpurgo, D. Natelson and J.-M. Triscone, Rev. Mod. Phys. 78(4) 1185 (2006)

[93] D. Cloetta, D. Ariosa, C. Cancellieri, M. Abrecht, S. Mitrovic and D. Pavuna, Phys. Rev. B 74014519 (2006)

[94] Y. Bruckental, A. Shaulov and Y. Yeshurun, Physica C Supercond. 460-462 761-763 (2007)

[95] E. Hanamura, Phys. Status Solidi B 234(1) 166-171 (2002)

[96] M. Matsuura, P. Dai, H. J. Kang, J. W. Lynn, D. N. Argyriou, K. Prokes, Y. Onose and Y. Tokura, Phys. Rev. B 68144503 (2003)

[97] G. Roberge, S. Charpentier, S. Godin-Proulx, P. Rauwel, K. Truong and P. Fournier, J. Cryst. Growth 311(5) 1340-1345 (2009)

[98] D. P. Beesabathina, L. Salamanca-Riba, S. N. Mao, X. X. Xi and T. Venkatesan, Appl. Phys. Lett. 62(23) 3022-3024 (1993) 
[99] S. N. Mao, X. X. Xi, Q. Li, T. Venkatesan, D. P. Beesabathina, L. Salamanca-Riba and X. D. Wu, J. Appl. Phys. 75(4) 2119-2124 (1994)

[100] M. Naito, S. Karimoto and A. Tsukada, Supercond. Sci. Technol. 15(12) $1663(2002)$

[101] A. Guarino, R. Fittipaldi, A. Romano, A. Vecchione and A. Nigro, Thin Solid Films $\mathbf{5 2 4}$ 282-289 (2012)

[102] B. D. Cullity, Elements of X-ray diffraction, Addison-Wesley (1956)

[103] D. B. Williams and C. B. Carter, Transmission Electron Microscopy: A Textbook for Material Science, New York: Springer (2009)

[104] S. Kubo and M. Suzuki, Physica C Supercond. 185-189 1251-1252 (1991)

[105] W. Jiang, S. N. Mao, X. X. Xi, X. Jiang, J. L. Peng, T. Venkatesan, C. J. Lobb and R. L. Greene, Phys. Rev. Lett. 73 1291-1294 (1994)

[106] S. Charpentier, G. Roberge, S. Godin-Proulx, X. Béchamp-Laganière, K. D. Truong, P. Fournier and P. Rauwel, Phys. Rev. B 81104509 (2010)

[107] R. Vaglio, C. Attanasio, L. Maritato and A. Ruosi, Phys. Rev. B 47(22) 15302 (1993)

[108] A. Gupta, G. Koren, C. C. Tsuei, A. Segmüller and T. R. McGuire, Appl. Phys. Lett. 55(17) 1795-1797 (1989)

[109] M. Brinkmann, T. Rex, M. Stief, H. Bach and K. Westerholt, Physica C Supercond. 269(1-2) 76-82 (1996)

[110] S. N. Mao, W. Jiang, X. X. Xi, Q. Li, J. L. Peng, R. L. Greene, T. Venkatesan, D. P. Beesabathina, L. Salamanca-Riba and X. D. Wu, Appl. Phys. Lett. 66(16) 2137-2139 (1995)

[111] D. H. A. Blank and H. Rogalla, J. Mater. Res. 12 2952-2957 (1997)

[112] J. G. Wen, N. Koshizuka, S. Tanaka, T. Satoh, M. Hidaka and S. Tahara, Appl. Phys. Lett. 75(16) 2470-2472 (1999)

[113] D. A. Muller, N. Nakagawa, A. Ohtomo, J. L. Grazul and H. Y. Hwang, Nature 430(7000) 657-661 (2004)

[114] B. Renker, F. Gompf, E. Gering, N. Nücker, D. Ewert, W. Reichardt and H. Rietschel, Z. Phys. B 67(1) 15-18 (1987)

[115] J. G. Simmons, J. Appl. Phys. 34(6) 1793-1803 (1963)

[116] W. L. McMillan and J. M. Rowell, Phys. Rev. Lett. 14 108-112 (1965)

[117] D. H. Wu, J. Mao, S. N. Mao, J. L. Peng, X. X. Xi, T. Venkatesan, R. L. Greene and S. M. Anlage, Phys. Rev. Lett. 70 85-88 (1993)

[118] T. Ekino, Physica C Supercond. 205(3-4) 338-346 (1993)

[119] T. Miyake, T. Imaizumi and I. Iguchi, Phys. Rev. B 68214520 (2003) 
[120] H. Shim, P. Chaudhari, G. Logvenov and I. Bozovic, Phys. Rev. Lett. 101 247004 (2008)

[121] J. W. Lynn, I. W. Sumarlin, D. A. Neumann, J. J. Rush, J. L. Peng and Z. Y. Li, Phys. Rev. Lett. 66 919-922 (1991)

[122] M.-Y. Shen, S.-L. Zhang and Y.-F. Zhang, Physica C Supercond. 196(1-2) 95-97 (1992)

[123] Q. Huang, J. F. Zasadzinski, N. Tralshawala, K. E. Gray, D. G. Hinks, J. L. Peng and R. L. Greene, Nature 347(6291) 369-372 (1990)

[124] M. Faley, U. Poppe, R. Dunin-Borkowski, M. Schiek, F. Boers, H. Chocholacs, J. Dammers, E. Eich, N. Shah, A. Ermakov, V. Slobodchikov, Y. Maslennikov and V. Koshelets, IEEE Trans. Appl. Supercond. 23(3) 1600705 (2013)

[125] D. Dimos, P. Chaudhari and J. Mannhart, Phys. Rev. B 41(7) 4038-4049 (1990)

[126] H. Hilgenkamp and J. Mannhart, Rev. Mod. Phys. 74(2) 485-549 (2002)

[127] D. Varshney, G. S. Patel and R. K. Singh, Supercond. Sci. Technol. 13(9) 1345-1355 (2000)

[128] W. J. Padilla, Y. S. Lee, M. Dumm, G. Blumberg, S. Ono, K. Segawa, S. Komiya, Y. Ando and D. N. Basov, Phys. Rev. B 72(6) 060511 (2005)

[129] R. C. Dynes, J. P. Garno, G. B. Hertel and T. P. Orlando, Phys. Rev. Lett. $532437-2440$ (1984)

[130] A. Pleceník, M. Grajcar, Š. Beňačka, P. Seidel and A. Pfuch, Phys. Rev. B 49 10016-10019 (1994)

[131] L. Glazman and K. Matveev, Sov. Phys. JETP 67 1276-1282 (1988)

[132] J. G. Simmons, J. Appl. Phys. 35(9) 2655-2658 (1964)

[133] A. A. Golubov, M. Y. Kupriyanov and E. Il'ichev, Rev. Mod. Phys. 76 411469 (2004)

[134] X. J. Zhou, T. Yoshida, A. Lanzara, P. V. Bogdanov, S. A. Kellar, K. M. Shen, W. L. Yang, F. Ronning, T. Sasagawa, T. Kakeshita, T. Noda, H. Eisaki, S. Uchida, C. T. Lin, F. Zhou, J. W. Xiong, W. X. Ti, Z. X. Zhao, A. Fujimori, Z. Hussain and Z.-X. Shen, Nature 423(6938) 398-398 (2003)

[135] A. Brinkman and H. Hilgenkamp, Physica C Supercond. 422(3-4) 71-75 (2005)

[136] I. Tsukada and S. Ono, Phys. Rev. B 74134508 (2006)

[137] H.-J. H. Smilde, H. Hilgenkamp, G. Rijnders, H. Rogalla and D. H. A. Blank, Appl. Phys. Lett. 80(24) 4579-4581 (2002)

[138] A. Khandale and S. Bhoga, Solid State Ionics 182(1) 82-90 (2011) 
[139] E. Paulus, I. Yehia, H. Fuess, J. Rodriguez, T. Vogt, J. Stroebel, M. Klauda and G. Saemann-Ischenko, Solid State Commun. 73(11) 791-795 (1990)

[140] P. G. Radaelli, D. G. Hinks, A. W. Mitchell, B. A. Hunter, J. L. Wagner, B. Dabrowski, K. G. Vandervoort, H. K. Viswanathan and J. D. Jorgensen, Phys. Rev. B 49(6) 4163-4175 (1994)

[141] J. Stangl, C. Mocuta, A. Diaz, T. H. Metzger and G. Bauer, ChemPhysChem 10(17) 2923-2930 (2009)

[142] M. Holt, K. Hassani and M. Sutton, Phys. Rev. Lett. 95(8) 085504 (2005)

[143] Y. Xiao, Z. Cai and B. Lai, Nanotechnology 16(9) 1754 (2005)

[144] M. Hanke, M. Dubslaff, M. Schmidbauer, T. Boeck, S. Schöder, M. Burghammer, C. Riekel, J. Patommel and C. G. Schroer, Appl. Phys. Lett. 92(19) 193109 (2008)

[145] M. Fratini, N. Poccia, A. Ricci, G. Campi, M. Burghammer, G. Aeppli and A. Bianconi, Nature 466(7308) 841-844 (2010)

[146] N. Hrauda, J. Zhang, E. Wintersberger, T. Etzelstorfer, B. Mandl, J. Stangl, D. Carbone, V. Holý, V. Jovanović, C. Biasotto, L. K. Nanver, J. Moers, D. Grützmacher and G. Bauer, Nano Lett. 11(7) 2875-2880 (2011)

[147] S. O. Hruszkewycz, C. M. Folkman, M. J. Highland, M. V. Holt, S. H. Baek, S. K. Streiffer, P. Baldo, C. B. Eom and P. H. Fuoss, Appl. Phys. Lett. 99(23) 232903 (2011)

[148] J. A. Klug, M. V. Holt, R. N. Premnath, A. Joshi-Imre, S. Hong, R. S. Katiyar, M. J. Bedzyk and O. Auciello, Appl. Phys. Lett. 99(5) 052902 (2011)

[149] N. Poccia, A. Ricci, G. Campi, M. Fratini, A. Puri, D. D. Gioacchino, A. Marcelli, M. Reynolds, M. Burghammer, N. L. Saini, G. Aeppli and A. Bianconi, PNAS 109(39) 15685-15690 (2012)

[150] C. V. Falub, M. Meduna, D. Chrastina, F. Isa, A. Marzegalli, T. Kreiliger, A. G. Taboada, G. Isella, L. Miglio, A. Dommann and H. von Kanel, Sci. Rep. 32276 (2013)

[151] M. Hoek, F. Coneri, D. P. Leusink, P. D. Eerkes, X. R. Wang and H. Hilgenkamp, Supercond. Sci. Technol. 27044017 (2014)

[152] A. Ricci, N. Poccia, G. Campi, F. Coneri, A. S. Caporale, D. Innocenti, M. Burghammer, M. von Zimmermann and A. Bianconi, Sci. Rep. 32383 (2013)

[153] S. K. Streiffer, B. M. Lairson and J. C. Bravman, Appl. Phys. Lett. 57(23) 2501-2503 (1990)

[154] B. W. Dodson, D. R. Myers, A. K. Datye, V. S. Kaushik, D. L. Kendall and B. Martinez-Tovar, Phys. Rev. Lett. 61 2681-2684 (1988)

[155] F. Riesz, Vacuum 46(8-10) 1021-1023 (1995) 
[156] A. Yamada, P. J. Fons, R. Hunger, K. Iwata, K. Matsubara and S. Niki, Appl. Phys. Lett. 79(5) 608-610 (2001)

[157] L. Wan, J. Shangguan, H. Luo, Y. Huang and B. Tang, The European Physical Journal - Applied Physics 38(03) 231-238 (2007)

[158] J. Gao, Y. Boguslavskij, B. B. G. Klopman, D. Terpstra, G. J. Gerritsma and H. Rogalla, Appl. Phys. Lett. 59(21) 2754-2756 (1991)

[159] J. Wen, N. Koshizuka, C. Traeholt, H. Zandbergen, E. Reuvekamp and H. Rogalla, Physica C Supercond. 255(3-4) 293-305 (1995)

[160] A. Fujimaki, Y. Fukai, M. Inoue, T. Kubo, L. Gómez and A. Maeda, Applied Superconductivity Conference (2006)

[161] L. Gómez, T. Kubo, H. Kitano, Y. Fukai, M. Inoue, A. Maeda and A. Fujimaki, The 4th International Symposium on Nanotechnology (2006)

[162] M. Honig, J. A. Sulpizio, J. Drori, A. Joshua, E. Zeldov and S. Ilani, Nat. Mater. 12(12) 1112-1118 (2013)

[163] G. Catalan, A. Lubk, A. H. G. Vlooswijk, E. Snoeck, C. Magen, A. Janssens, G. Rispens, G. Rijnders, D. H. A. Blank and B. Noheda, Nat. Mater. 10(12) 963-967 (2011)

[164] R. J. Zeches, M. D. Rossell, J. X. Zhang, A. J. Hatt, Q. He, C.-H. Yang, A. Kumar, C. H. Wang, A. Melville, C. Adamo, G. Sheng, Y.-H. Chu, J. F. Ihlefeld, R. Erni, C. Ederer, V. Gopalan, L. Q. Chen, D. G. Schlom, N. A. Spaldin, L. W. Martin and R. Ramesh, Science 326(5955) 977-980 (2009)

[165] J. X. Zhang, Q. He, M. Trassin, W. Luo, D. Yi, M. D. Rossell, P. Yu, L. You, C. H. Wang, C. Y. Kuo, J. T. Heron, Z. Hu, R. J. Zeches, H. J. Lin, A. Tanaka, C. T. Chen, L. H. Tjeng, Y.-H. Chu and R. Ramesh, Phys. Rev. Lett. 107147602 (2011)

[166] I. C. Infante, J. Juraszek, S. Fusil, B. Dupé, P. Gemeiner, O. Diéguez, F. Pailloux, S. Jouen, E. Jacquet, G. Geneste, J. Pacaud, J. Íñiguez, L. Bellaiche, A. Barthélémy, B. Dkhil and M. Bibes, Phys. Rev. Lett. 107 237601 (2011)

[167] M. Y. Chern, A. Gupta and B. W. Hussey, Appl. Phys. Lett. 60(24) 30453047 (1992)

[168] M.-J. Casanove, A. Alimoussa, M. Schwerdtfeger, S. Gaubert, H. Moriceau and J.-C. Villegier, Materials Science and Engineering: B 33(2-3) 162-167 (1995)

[169] G. Rietveld, M. Glastra and D. van der Marel, Physica C Supercond. 241(34) $257-272(1995)$

[170] I. Bozovic, G. Logvenov, I. Belca, B. Narimbetov and I. Sveklo, Phys. Rev. Lett. 89107001 (2002)

[171] J. P. Eisenstein, L. N. Pfeiffer and K. W. West, Appl. Phys. Lett. 57(22) $2324-2326$ (1990) 
[172] C. T. Chen, L. H. Tjeng, J. Kwo, H. L. Kao, P. Rudolf, F. Sette and R. M. Fleming, Phys. Rev. Lett. 68 2543-2546 (1992)

[173] E. Pellegrin, N. Nücker, J. Fink, S. L. Molodtsov, A. Gutiérrez, E. Navas, O. Strebel, Z. Hu, M. Domke, G. Kaindl, S. Uchida, Y. Nakamura, J. Markl, M. Klauda, G. Saemann-Ischenko, A. Krol, J. L. Peng, Z. Y. Li and R. L. Greene, Phys. Rev. B 47(6) 3354-3367 (1993)

[174] A. Y. Ignatov, A. A. Ivanov, A. P. Menushenkov, S. Iacobucci and P. Lagarde, Phys. Rev. B 57 8671-8679 (1998)

[175] M. Grioni, J. B. Goedkoop, R. Schoorl, F. M. F. de Groot, J. C. Fuggle, F. Schäfers, E. E. Koch, G. Rossi, J.-M. Esteva and R. C. Karnatak, Phys. Rev. B 39 1541-1545 (1989)

[176] J. Fink, N. Nücker, E. Pellegrin, H. Romberg, M. Alexander and M. Knupfer, J. Electron. Spectrosc. Relat. Phenom. 66(3-4) 395-452 (1994)

[177] N. Nücker, E. Pellegrin, P. Schweiss, J. Fink, S. L. Molodtsov, C. T. Simmons, G. Kaindl, W. Frentrup, A. Erb and G. Müller-Vogt, Phys. Rev. $B 518529-8542$ (1995)

[178] M. Merz, N. Nücker, P. Schweiss, S. Schuppler, C. T. Chen, V. Chakarian, J. Freeland, Y. U. Idzerda, M. Kläser, G. Müller-Vogt and T. Wolf, Phys. Rev. Lett. 80 5192-5195 (1998)

[179] M. Grioni, J. F. van Acker, M. T. Czyžyk and J. C. Fuggle, Phys. Rev. B 45 3309-3318 (1992)

[180] P. W. Hillyard, S. V. N. T. Kuchibhatla, T. E. Glover, M. P. Hertlein, N. Huse, P. Nachimuthu, L. V. Saraf, S. Thevuthasan and K. J. Gaffney, Phys. Rev. B 80125210 (2009)

[181] A. Kirfel and K. Eichhorn, Acta Crystallographica Section A 46(4) 271-284 (1990)

[182] S. Sachdev, Annual Review of Condensed Matter Physics 3(1) 9-33 (2012)

[183] J. Zaanen, Nat Phys 9(10) 609-610 (2013)

[184] A. J. Beekman and J. Zaanen, Phys. Rev. B 86(12) 125129 (2012)

[185] A. J. Beekman, Vortex duality in higher dimensions, Ph.D. thesis, Leiden University (2011)

[186] T. Timusk and B. Statt, Reports on Progress in Physics 62(1) 61 (1999)

[187] T. Valla, A. V. Fedorov, J. Lee, J. C. Davis and G. D. Gu, Science 314(5807) 1914-1916 (2006)

[188] J. Lee, K. Fujita, A. R. Schmidt, C. K. Kim, H. Eisaki, S. Uchida and J. C. Davis, Science 325(5944) 1099-1103 (2009)

[189] T. Kondo, Y. Hamaya, A. D. Palczewski, T. Takeuchi, J. S. Wen, Z. J. Xu, G. Gu, J. Schmalian and A. Kaminski, Nat Phys 7(1) 21-25 (2011) 
[190] J. Corson, R. Mallozzi, J. Orenstein, J. N. Eckstein and I. Bozovic, Nature 398(6724) 221-223 (1999)

[191] Z. A. Xu, N. P. Ong, Y. Wang, T. Kakeshita and S. Uchida, Nature 406(6795) 486-488 (2000)

[192] Y. Wang, L. Li, M. J. Naughton, G. D. Gu, S. Uchida and N. P. Ong, Phys. Rev. Lett. 95247002 (2005)

[193] C. C. Homes, S. V. Dordevic, M. Strongin, D. A. Bonn, R. Liang, W. N. Hardy, S. Komiya, Y. Ando, G. Yu, N. Kaneko, X. Zhao, M. Greven, D. N. Basov and T. Timusk, Nature 430(6999) 539-541 (2004)

[194] I. Bozovic, G. Logvenov, M. A. J. Verhoeven, P. Caputo, E. Goldobin and M. R. Beasley, Phys. Rev. Lett. 93(15) 157002 (2004)

[195] E. Morenzoni, B. M. Wojek, A. Suter, T. Prokscha, G. Logvenov and I. Bozovic, Nat Commun 2272 (2011)

[196] B. Nachumi, A. Keren, K. Kojima, M. Larkin, G. M. Luke, J. Merrin, O. Tchernyshöv, Y. J. Uemura, N. Ichikawa, M. Goto and S. Uchida, Phys. Rev. Lett. 77 5421-5424 (1996)

[197] H. H. Wen, H. P. Yang, S. L. Li, X. H. Zeng, A. A. Soukiassian, W. D. Si and X. X. Xi, Europhys. Lett. 64(6) 790 (2003)

[198] A. Malinowski, M. Z. Cieplak and M. Berkowski, J. Phys.: Condens. Matter 20(8) 085207 (2008)

[199] G. E. Blonder, M. Tinkham and T. M. Klapwijk, Phys. Rev. B 25(7) 45154532 (1982)

[200] V. Gantmakher, Electrons and Disorder in Solids, The international series of monographs on physics, Clarendon Press Oxford (2005)

[201] M. V. Jacob, A. Sundaresan, J. Mazierska and Y. Tanaka, IEEE Trans. Appl. Supercond. 15(2) 3596-3599 (2005)

[202] A. T. Bollinger, G. Dubuis, J. Yoon, D. Pavuna, J. Misewich and I. Bozovic, Nature 472(7344) 458-460 (2011)

[203] X. Leng, J. Garcia-Barriocanal, S. Bose, Y. Lee and A. M. Goldman, Phys. Rev. Lett. 107027001 (2011)

[204] Y. Mizugaki, Y. Uematsu, S.-J. Kim, J. Chen, K. Nakajima, T. Yamashita, H. Sato and M. Naito, J. Appl. Phys. 94(4) 2534-2537 (2003)

[205] A. Gozar, G. Logvenov, L. F. Kourkoutis, A. T. Bollinger, L. A. Giannuzzi, D. A. Muller and I. Bozovic, Nature 455(7214) 782-785 (2008)

[206] L. Rademaker, J. Zaanen and H. Hilgenkamp, Phys. Rev. B 83012504 (2011)

[207] R. Pentcheva, M. Huijben, K. Otte, W. E. Pickett, J. E. Kleibeuker, J. Huijben, H. Boschker, D. Kockmann, W. Siemons, G. Koster, H. J. W. Zandvliet, G. Rijnders, D. H. A. Blank, H. Hilgenkamp and A. Brinkman, Phys. Rev. Lett. 104166804 (2010) 
[208] M. Huijben, D. Kockmann, J. Huijben, J. E. Kleibeuker, A. van Houselt, G. Koster, D. H. A. Blank, H. Hilgenkamp, G. Rijnders, A. Brinkman and H. J. W. Zandvliet, Phys. Rev. B 86035140 (2012)

[209] Y. Hotta, T. Susaki and H. Y. Hwang, Phys. Rev. Lett. 99236805 (2007)

[210] A. Ohtomo, D. A. Muller, J. L. Grazul and H. Y. Hwang, Nature 419(6905) 378-380 (2002)

[211] P. Moetakef, T. A. Cain, D. G. Ouellette, J. Y. Zhang, D. O. Klenov, A. Janotti, C. G. Van de Walle, S. Rajan, S. J. Allen and S. Stemmer, Appl. Phys. Lett. 99(23) 232116 (2011)

[212] A. Janotti, L. Bjaalie, B. Himmetoglu and C. G. Van de Walle, Phys. Status Solidi RRL 1-609088 (2014)

[213] M. Kellogg, J. P. Eisenstein, L. N. Pfeiffer and K. W. West, Phys. Rev. Lett. 93(3) 036801 (2004)

[214] A. F. Croxall, K. Das Gupta, C. A. Nicoll, M. Thangaraj, H. E. Beere, I. Farrer, D. A. Ritchie and M. Pepper, Phys. Rev. Lett. 101(24) 246801 (2008)

[215] S. Thiel, G. Hammerl, A. Schmehl, C. W. Schneider and J. Mannhart, Science 313(5795) 1942-1945 (2006)

[216] P. D. Eerkes, W. G. van der Wiel and H. Hilgenkamp, Appl. Phys. Lett. 103(20) 201603 (2013)

[217] B. Seradjeh, J. E. Moore and M. Franz, Phys. Rev. Lett. 103(6) 066402 (2009)

[218] X.-L. Qi and S.-C. Zhang, Physics Today 63(1) 33-38 (2010)

[219] B. Yan, C. Felser and S.-C. Zhang, Phys. Status Solidi RRL 7(1-2) 13-14 (2013)

[220] M. Veldhorst, M. Snelder, M. Hoek, T. Gang, V. K. Guduru, X. L. Wang, U. Zeitler, W. G. van der Wiel, A. A. Golubov, H. Hilgenkamp and A. Brinkman, Nat. Mater. 11(5) 417-421 (2012)

[221] M. Veldhorst, M. Snelder, M. Hoek, C. G. Molenaar, D. P. Leusink, A. A. Golubov, H. Hilgenkamp and A. Brinkman, Phys. Status Solidi RRL 7(1-2) 26-38 (2013)

[222] M. Veldhorst, M. Hoek, M. Snelder, H. Hilgenkamp, A. A. Golubov and A. Brinkman, Phys. Rev. B 90035428 (2014)

[223] L. Fu and C. L. Kane, Phys. Rev. B 79161408 (2009)

[224] N. Tombros, A. Veligura, J. Junesch, J. Jasper van den Berg, P. J. Zomer, M. Wojtaszek, I. J. Vera Marun, H. T. Jonkman and B. J. van Wees, J. Appl. Phys. 109(9) 093702 (2011)

[225] P. Rickhaus, R. Maurand, M.-H. Liu, M. Weiss, K. Richter and C. Schönenberger, Nat Commun 42342 (2013) 


\section{Propositions}

accompanying the thesis

\section{At the interface between electron and hole-doped cuprates}

\section{Marcel Hoek}

1. The presence or absence of the $(R E, \mathrm{Ce})_{2} \mathrm{O}_{3}(R E=\mathrm{Pr}, \mathrm{Nd}, \ldots)$ parasitic phase in the electron-doped cuprates plays an important role during vacuum reduction.

(chapter 2)

2. The cleanest interface between $\mathrm{Nd}_{2-\mathrm{x}} \mathrm{Ce}_{\mathrm{x}} \mathrm{CuO}_{4}$ and $\mathrm{La}_{2-\mathrm{x}} \mathrm{Sr}_{\mathrm{x}} \mathrm{CuO}_{4}$ is made using a ramp-edge junction.

(chapters 3-5)

3. Ramp-edge technology can be used to create artificial domain structures.

(chapter 4)

4. The lack of good formal training in presenting scientific work and public speaking in science stands in the way of progress.

5. Hand waving arguments are easily convincing, but difficult to prove right.

6. It is better for the understanding of the audience when mathematical derivations are not explained using Powerpoint.

7. The Dutch system of national holidays is due for a major overhaul.

8. Traffic safety will be improved if yielding your right of way is forbidden. 
behorende bij het proefschrift

\title{
At the interface between electron and hole-doped cuprates
}

\author{
Marcel Hoek
}

1. De aan- of afwezigheid van de $(R E, \mathrm{Ce})_{2} \mathrm{O}_{3}(R E=\mathrm{Pr}, \mathrm{Nd}, \ldots)$ parasitaire fase in de elektronen-gedoteerde cupraten speelt een belangrijke rol tijdens de vacuümbehandeling.

(hoofdstuk 2)

2. Het schoonste grensvlak tussen $\mathrm{Nd}_{2-\mathrm{x}} \mathrm{Ce}_{\mathrm{x}} \mathrm{CuO}_{4}$ en $\mathrm{La}_{2-\mathrm{x}} \mathrm{Sr}_{\mathrm{x}} \mathrm{CuO}_{4}$ wordt gemaakt met behulp van helling-type juncties.

(hoofdstukken 3-5)

3. Helling-type juncties kunnen gebruikt worden om kunstmatige domeinstructuren te realiseren.

(hoofdstuk 4)

4. Het gebrek aan een gedegen training in het presenteren van wetenschappelijk werk en spreken in het openbaar in de wetenschap staat vooruitgang in de weg.

5. Redeneringen uit de losse pols zijn snel overtuigend, maar moeilijk daadwerkelijk te bewijzen.

6. Het is voor het begrip van het publiek beter wanneer wiskundige afleidingen niet uitgelegd worden met behulp van Powerpoint.

7. Het Nederlandse systeem van nationale feestdagen is toe aan een grondige herziening.

8. Het komt de verkeersveiligheid ten goede als voorrang geven terwijl je voorrang hebt verboden wordt. 